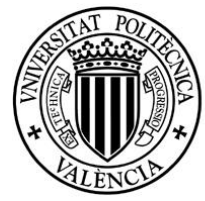

UNIVERSITAT

POLITĖCNICA

DE VALĖNCIA

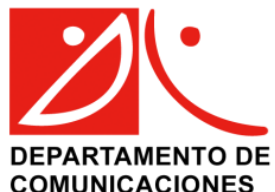

Universidad Politécnica de Valencia Departamento de Comunicaciones

\title{
Coexistencia e Integración de Comunicaciones Inalámbricas en Sistemas de Transmisión Ópticos
}

Tesis Doctoral

realizada por

D. Joaquín Pérez Soler

dirigida por

Dr. Roberto Llorente Sáez

Valencia, Julio del 2009

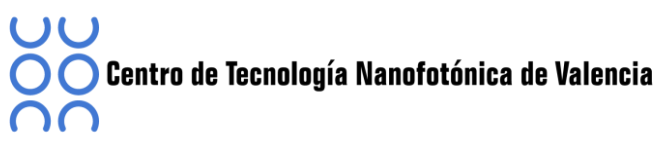





\section{Resumen}

Las redes y sistemas de telecomunicación actuales se enfrentan a la necesidad de proporcionar regímenes binarios en la red de acceso cada vez más elevados a un mayor número de usuarios. Esta necesidad se basa en el aumento de tráfico de Internet, relacionado con la demanda y distribución de programas y videojuegos en línea, con el aumento de la complejidad en los contenidos de las páginas web, con la distribución de contenidos audiovisuales y contenidos en línea adicionales, y con la introducción de la alta definición en los servicios y contenidos distribuidos, todo ello consecuencia de una sociedad cada vez más interconectada. A fin de satisfacer la demanda de mayores regímenes binarios en la red de acceso, esta Tesis Doctoral propone y demuestra nuevas técnicas que permiten la distribución eficiente de señales de radiofrecuencia correspondientes a distintos sistemas de comunicaciones inalámbricos de manera conjunta. Las técnicas propuestas tienen como objetivo facilitar una red de acceso integrada en el entorno del usuario, es decir, integrar en una misma infraestructura la transmisión óptica en la red óptica de acceso y posteriormente la transmisión vía radiofrecuencia.

Los sistemas de comunicación inalámbricos considerados en esta Tesis Doctoral son el sistema WiMAX (Worldwide Interoperability for Microwave Access) y la tecnología de transmisión de banda ultra-ancha UWB (Ultra-Wide Band), basada en la definición dada por WiMedia Alliance. Estos sistemas son altamente complementarios entre sí, por lo que es interesante facilitar su provisión conjunta en redes de acceso integradas. La red óptica de acceso considerada en esta Tesis Doctoral se corresponde con una red de fibra hasta el hogar (FTTH, Fibre-to-the-Home). Sobre esta red óptica de acceso se realiza la transmisión de las señales de los sistemas inalámbricos en su formato original, es decir, sin remodulaciones ni conversiones de frecuencia, en una o varias portadoras ópticas. Esta tecnología, denominada radio-sobre-fibra (RoF, Radio-over-Fibre), es especialmente interesante en redes de acceso integradas.

En primer lugar, esta Tesis Doctoral estudia los requisitos para la convergencia inalámbrica de servicios basados en MB-OFDM UWB (Multi-Band 
Orthogonal-Frequency Division-Multiplexing UWB) y WiMAX 802.16e en entornos de redes inalámbricas personales (WPAN, Wireless Personal Area Network). El estudio y evaluación experimental de esta convergencia tiene como objetivo proporcionar márgenes de seguridad para asegurar la interoperabilidad entre ambas tecnologías radio, los cuales pueden aplicarse en el futuro desarrollo de tecnologías avanzadas de mitigación de la interferencia como detección y exclusión (DAA, Detect-and-Avoid) en el marco de futuras aplicaciones como cognitive radio.

A continuación, se aborda la coexistencia de MB-OFDM UWB y WiMAX en redes ópticas de acceso utilizando RoF. Se estudian dos escenarios de aplicación: en el primero se evalúa un sistema RoF sobre fibra multimodo, y en el segundo, un sistema RoF sobre fibra monomodo estándar. Los resultados obtenidos permiten delimitar el alcance en cada tipo de medio óptico para el despliegue de sistemas RoF sobre FTTH. A su vez, se propone y demuestra experimentalmente una nueva técnica de multiplexación por división de polarización en la transmisión óptica que facilita la coexistencia en RoF.

Finalmente, esta Tesis Doctoral estudia el modulador electro-óptico Mach-Zehnder como dispositivo clave que limita las prestaciones de las transmisiones en sistemas RoF. A su vez, se propone y demuestra experimentalmente una técnica de linealización que permite aumentar el margen dinámico de este modulador. 


\section{Resum}

Els actuals sistemes de telecomunicació deuen afrontar la necessitat de proporcionar règims binaris força elevats a les xarxes d'accés a una gran quantitat d'usuaris. Aquesta demanda es basa, principalment, en l'augment del trànsit d'Internet, relacionat amb la distribució de programes i videojocs en línia, amb l'augment de la complexitat dels continguts de les pàgines web, amb la distribució de continguts audiovisuals i addicionals en línia, i amb la introducció de l'alta definició en els serveis i continguts distribuïts, tot això com a consequiència d'una societat cada vegada més interconnectada. Per tal de respondre a aquesta demanda de règims binaris força elevats, aquesta Tesi Doctoral proposa i desenvolupa noves tècniques per a la distribució eficient dels senyals de diversos sistemes de comunicacions sense fils de forma conjunta. L'objectiu d'aquestes tècniques és proporcionar una xarxa d'accés integrada a l'usuari final, és a dir, integrant a la mateixa infraestructura la transmissió òptica a la xarxa òptica d'accés i la darrera transmissió via radiofreqüència.

Aquesta Tesi Doctoral considera dos tipus de sistemes de comunicacions sense fils, WiMAX (Worldwide Interoperability for Microwave Access) i UWB (Ultra-Wide Band) segons la definició realitzada per WiMedia Alliance. A partir de les característiques de règim binari i abast, aquests dos sistemes d'alta capacitat es defineixen com a complementaris. Per tant, facilitar la seua distribució conjunta en xarxes integrades d'accés es considera de gran interés. En aquesta Tesi Doctoral, la xarxa òptica d'accés es correspon amb una xarxa de fibra fins a la llar (FTTH, Fibre-to-the-Home). En aquesta xarxa òptica d'accés, la transmissió del senyal dels sistemes sense fils es realitza en la seua forma original, sense re-modulacions ni conversió en freqüència, en una o vàries portadores òptiques. Aquesta tecnologia s'anomena ràdio- sobre- fibra ( $\mathrm{RoF}$, Radio-over-Fibre).

En primer lloc, aquesta Tesi Doctoral estudia els requeriments per a la convergència de serveis fonamentats en les tecnologies sense fils MB-OFDM UWB (Multi-Band Orthogonal-Frequency Division-Multiplexing UWB) i WiMAX IEEE 802.16e en xarxes personals de comunicacions sense fils (WPAN, Wireless 
Personal Area Network). L'objectiu d'aquest estudi és obtenir màrges de seguretat per a garantir la interoperatibilitat d'ambdues tecnologies ràdio, els quals es poden aplicar en el futur desenvolupament de tècniques avançades de mitigació de la interferència com detecció i exclusió (DAA, Detect-and-Avoid), dins de futures aplicacions com les anomenades cognitive radio.

Després, s'estudia la coexistència de MB-OFDM UWB i WiMAX IEEE 802.16e en xarxes òptiques d'accés basades en sistemes RoF. S'avaluen dos escenaris experimentals: en el primer, s'avalua un sistema RoF amb fibra multimode, i el segon, un sistema RoF amb fibra monomode. Els resultats permeten obtenir màrges de seguretat $\mathrm{i}$ abast màxim per al desplegament de sistemes RoF segons els tipus de fibra. Al mateix temps, es demostra $\mathrm{i}$ avalua experimentalment una nova tècnica de multiplexació per divisió en polarització de la transmissió òptica, per a millorar la coexistència en sistemes RoF.

Finalment, aquesta Tesi Doctoral presenta l'estudi del modulador electro-òptic Mach-Zehnder, com a peça clau que limita el marge dinàmic de les transmissions als sistemes RoF. Al mateix temps, es proposa i avalua experimentalment una tècnica òptica de linealització del modulador Mach-Zehnder que millora el marge dinàmic del sistema RoF. 


\section{Abstract}

Current network and telecommunication systems are required to provide higher data rates in access networks to an increasing number of users. This fact is mainly due to the increase in the Internet traffic data, which is related with the higher demand of online videogames and software, the increased complexity in the content of web pages, the joint distribution of audio-visual and added-value online content, and the introduction of high-definition services and contents such as video on demand, as a result of a society increasingly more interconnected. In order to satisfy these higher data rates requirements, new techniques for the joint distribution of several wireless communication systems are proposed in this Thesis. The aim of these techniques is to facilitate the deployment of an integrated access network at the customer premises, enabling the integration of optical transmission over an optical access network and radio-frequency transmission in the same infrastructure.

Two main wireless communication systems are considered in this Thesis, WiMAX (Worldwide Interoperability for Microwave Access) and UWB (Ultra-Wide Band) according to WiMedia Alliance recommendation. Comparing the bit rate and expected range, WiMAX and UWB are complementary radio technologies expected to coexist in a near future in integrated access networks. The optical access network considered in this Thesis can be regarded as a FTTH network (Fibre-to-the-Home). The wireless signals are natively transmitted over optical network, that is, without frequency upconversion and remodulation stages, over one or several optical carriers. This technology, which is known as Radio-over-Fibre (RoF), is well suited for integrated access networks.

First, the requirements for the wireless convergence of services based on Multi-Band Orthogonal-Frequency Division-Multiplexing UWB (MB-OFDM UWB) and WiMAX 802.16e in Wireless Personal Area Networks (WPAN) are stated. The aim of this study is to provide relevant protection margins in order to ensure the coexistence between both technologies. The obtained protection margins are of great interest for the 
development of advanced interference mitigation techniques such as DAA (Detect-and-Avoid), in the framework of future cognitive radio technologies.

In a second step, the wireless coexistence of MB-OFDM UWB and WiMAX technologies is analyzed from the point of view of access networks based on RoF systems. Two experimental field trials are here carried out. In the first one, the wireless convergence is evaluated in a multi-mode fibre RoF system, whereas in the second one, the RoF system is based on a standard single-mode fibre. These experimental results provide relevant fibre link transmission distances to enable the deployment of RoF networks. Moreover, a new optical transmission technique based on polarization division multiplexing is proposed and experimentally evaluated in order to ensure the wireless coexistence in RoF systems.

Finally, the impact of the electro-optical Mach-Zehnder modulator is analyzed, since the dynamic range of this device limits the performance of the RoF system. Moreover, a new optical linearization technique for Mach-Zehnder modulators is proposed and evaluated in order to overcome this limitation. 
A mi abuelito Paulino,

mi abuelita,

mi abuela Josefa

y al "palle" 



\section{Agradecimientos}

Quiero agradecer al Dr. Javier Martí Sendra, director del Instituto Centro de Tecnología Nanofotónica de Valencia (NTC), el haberme brindado la oportunidad de incorporarme al grupo radio-sobre-fibra del NTC y desarrollar así mi Tesis Doctoral. Y por supuesto, al Dr. Roberto Llorente Sáez por la dirección de mi trabajo y su apoyo durante estos años.

A su vez, no quiero olvidarme de todos los que son y han sido parte del grupo humano que forma este Instituto. En especial, quiero agradecer el apoyo de todos aquellos que me acogieron al comenzar en este Instituto, con cafés y futbol sala, y de mi grupo de investigación y sus horas de mesa óptica.

Dar las gracias de forma especial a mis padres, Ana y Joaquín María, y a mi hermano Israel por todo su apoyo en todos los días que ha costado llegar hasta aquí. Y recordar también al resto de mi familia, titas, titos, y todos esos primos, desde los que me sacan una sonrisa hasta los que siempre tienen una buena charla en cualquier momento.

Este trabajo también tiene recuerdos especiales para todos aquellos que con sus paellas, tiburones, montañas, cafés son parte de esta Tesis. Y para esos músicos de la Banda Sinfónica de la UPV, de mi pueblo y todos los de por allí abajo dal lao de mi casa, que siempre me escuchan entre notas que esto se acaba, muchas gracias. Y un recuerdo especial a la gente de mi otro pueblo, que también conocen que con su música y sus comidas todo es un poco más fácil, moltes gràcies Carmen, Vicent i Pablo.

Y esto llega hasta aquí porque tú has querido que llegue, porque no dejas que las cosas buenas se olviden, que el esfuerzo sea en balde y me ayudas a disfrutar siempre, afinado y en su sitio, y esa eres tú Carme, gràcies. 



\section{Lista de acrónimos}

3GPP

ADC

ADSL

ATM

$\mathrm{AV}$ ó A/V

AWG

B2B

BAN

BER

BG

BPF

BPON

BS

BWA

$\mathrm{C} 2 \mathrm{C}$

CATV

CDMA

CO

$\mathrm{CP}$

CPE
Third Generation Partnership Project

Analog-to-Digital Converter

Asymmetrical Digital Subscriber Line

Asynchronous Transfer Mode

Audio and Video

Arrayed Waveguide Grating

Back-to-Back

Body Area Network

Bit Error Rate

Band Group

Band-Pass Filter

Broadband-Passive Optical Network

Base Station

Broadband Wireless Access

Car-to-Car

Cable Television

Code Division Multiple Access

Central Office

Cyclic Prefix

Customer Premises Equipment 
CRRM

CS

CSO

CTB

CWUSB

DAA

DAN

DAS

DCF

DCM

DCM2P

DCoM

DFB

DL

DPMZM

DR

DSL

DSMZM

DS-SS

DS-UWB

DWDM

e2e

EAM

ECL

EDFA

EDGE

EFM

EGPRS

EIRP

$\mathrm{E} / \mathrm{O}$
Common Radio Resource Management

Central Station

Composite Second-Order Beat

Composite Triple Beat

Certified Wireless Universal Serial Bus

Detect-and-Avoid

Distributed Antenna Network

Distributed Antenna Systems

Dispersion-Compensating Fibre

Dual Carrier Modulation

Directional Coupler Modulator with Two Passive Bias

Sections in Cascade

Simple Directional Coupler Modulator

Distributed Feedback Laser

Downlink

Dual Parallel Mach-Zehnder Modulator

Dynamic Range

Digital Subscriber Line

Dual-Serial Mach-Zehnder Modulator

Direct-Sequence Spread Spectrum

Direct-Sequence Ultra-Wideband

Dense Wavelength Division Multiplexing

End-to-End

Electro-Absorption Modulator

External Cavity Laser

Erbium Doped Fibre Amplifier

Enhanced Data Rates for GSM Evolution

Ethernet in the First Mile

Enhanced General Packet Radio Service

Equivalent Isotropic Radiated Power

Electro-Optical 
EOM

EPON

EV-DO Rev. B

EVM

FBG

FCC

$\mathrm{FCH}$

FDD

FFI

FFT

FSAN

FTTB

FTTC

FTTCab

FTTE

FTTH

FTTN

FTTx

FWA

$\mathrm{GbE}$

GPON

GPR

GSM

GVD

HAN

HD

HD2

HD3

HDMI

HDTV
Electro-Optic Modulator

Ethernet Passive Optical Network

Evolved Data Optimized Revision B

Error-Vector Magnitude

Fibre Bragg Grating

Federal Communications Comission

Frame Control Header

Frequency-Division Duplexing

Fixed-Frequency Interleaved

Fast-Fourier Transform

Full Service Access Networks

Fibre-to-the-Building

Fibre-to-the-Curb

Fibre-to-the-Cabinet

Fibre-to-the-Enclosure

Fibre-to-the-Home

Fibre-to-the-Node/Neighbourhood

Fibre-to-the- $x$

Fixed Broadband Wireless Access

Gigabit Ethernet

Gigabit Ethernet Passive Optical Network

Ground Penetration Radar

Global System for Mobile communications

Group Velocity Dispersion

Human Area Network

High Definition

Harmonic Second Order Distortion

Harmonic Third Order Distortion

High-Definition Multimedia Interface

High-Definition Television 
HFC

HSDPA

HSPA

HSUPA

I2V

ICT

IL

IMD

IMD2

IMD3

IMS

IMT

IP

IPTV

IR-UWB

ISM

ITU-T

LAN

LDC

LED

LMDS

LNA

LOS

LTE

MAC

MATB

MB-OFDM

$\mathrm{MC}$

MIMO

MITB
Hybrid Fibre-Coaxial

High Speed Downlink Packet Access

High Speed Packet Access

High Speed Uplink Packet Access

Infrastructure-to-Vehicule

Information and Communications Technologies

Inline Amplification

Intermodulation Distortion

Intermodulation Second Order Distortion

Intermodulation Third Order Distortion

Internet Protocol Multimedia Subsystem

International Mobile Telecommunications

Internet Protocol

Internet Protocol Television

Impulse Radio UWB

Industrial, Scientifical and Medical

International Telecommunication Union - Telecommunication Standardization Sector

Local Area Network

Low Duty Cicle

Light Emitting Diode

Local Multipoint Distribution Service

Low Noise Amplifier

Line of Sight

Long Term Evolution

Medium Access Control

Maximum Transmission Bias

Multi-Band Orthogonal-Frequency Division-Multiplexing

Media Converters

Multiple-Input Multiple-Output

Minimum Transmission Bias 
MMF

MS

$\mathrm{MZ}$

MZ-EOM

MZM

NGMN

NGN

NLF

NLOS

$\mathrm{O} / \mathrm{E}$

OBPF

ODL

OFDM

OFDMA

OFDMA-TDD

OFM

OLT

ONT

ONU

PAL

PBS

PC

PCF-DCF

PCI

PCMCIA

PD

$\mathrm{PDH}$

PDM

PHY

PM
Multi-Mode Fibre

Mobile Station

Mach Zehnder

Mach-Zehnder Electro-Optic Modulator

Mach-Zehnder Modulator

Next Generation Mobile Network

Next Generation Networks

Non-Linear Fibre

Non-Light of Sight

Optical-to-Electrical

Optical Band-Pass Filter

Optical Delay Line

Orthogonal-Frequency Division Multiplexing

Orthogonal-Frequency Division Multiple Access

OFDMA Time-Division Duplexing

Optical Frequency Multiplication

Optical Line Terminal

Optical Network Terminal

Optical Network Unit

Protocol Adaptation Layer

Polarization Beam Splitter/Combiner

Polarization Controller

Photonic Crystal-Fibre Dispersion-Compensating Fibre

Peripheral Component Interconnect

Personal Computer Memory Card International Association

Photo-Diode

Plesiochronous Digital Hierarchy

Polarization Division Multiplexing

Physical Layer

Polarization-Multiplexed 
PM/MZM

PMD

POF

PON

PSD

PtMP

PtP

QAM

QB

QoS

QPSK

RBW

RCE

RF

RIN

RoF

RoMMF

RoSMF

RoSSMF

RSOA

RX

SCM

SDCM

$\mathrm{SDH}$

SFDR

SMF

SNR

SOFDMA

SONET

SSB
Phase Modulation Mach-Zehnder Modulator

Polarization Mode Dispersion

Plastic Optical Fibre

Passive Optical Network

Power Spectral Density

Point-to-Multipoint

Point-to-Point

Quadrature Amplitude Modulation

Quadrature BIAS

Quality of Service

Quadrature-Phase Shift Keying

Resolution Bandwidth

Relative Constellation Error

Radio-frequency

Relative Intensive Noise

Radio-over-Fibre

Radio-over-Multi-Mode Fibre

Radio-over-Single-Mode Fibre

Radio-over-Standard Single-Mode Fibre

Reflective Semiconductor Optical Amplifier

Receiver

Sub-Carrier Multiplexing

Sub-octave Directional Coupler Modulator

Synchronous Digital Hierarchy

Spurious-Free Dynamic Range

Single-Mode Fibre

Signal-to-Noise Ratio

Scalable OFDM Access

Synchronous Optical Network

Single-Side Band 
SSB-SC

SSMF

TDD

TDM

TFC

TFI

TFI2

TH-PPM

TIA

TM-UWB

TS

TX

UL

UMTS

USB

USB-IF

UWB

VCSEL

VDSL

VoIP

WCDMA

WDM

Wi-Fi

WiMAX

WLAN

WMAN

WPAN

WUSB

WWAN
Single-Side Band Supression Carrier

Standard Single-Mode Fibre

Time-Division Duplexing

Time-Division Multiplexing

Time-Frequency Code

Time-Frequency Interleaving

Two-band Time-Frequency Interleaving

Time Hopping Pulse Position Modulation

Transimpedance Amplifier

Time-Modulated Ultra-Wideband

Terminal Station

Transmitter

Uplink

Universal Mobile Telecommunications System

Universal Serial Bus

Universal Serial Bus Implementers Forum

Ultra-Wideband

Vertical-Cavity Surface-Emitting Laser

Very High Bitrate Digital Subscriber Line

Voice-over-IP

Wideband Code Division Multiple Access

Wavelength Division Multiplexing

Wireless Fidelity

Worldwide Interoperability for Microwave Access

Wireless Local Area Networks

Wireless Metropolitan Area Network

Wireless Personal Area Network

Wireless Universal Serial Bus

Wireless Wide Area Network 



\section{Índice general}

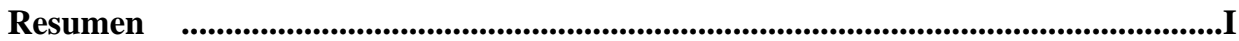

Resum _......................................................................................................... III

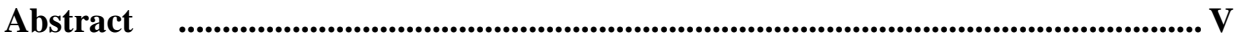

Lista de acrónimos ................................................................................................................XI

Índice general .....................................................................................................................XIX

Índice de figuras..................................................................................................XXIII

Índice de tablas.........................................................................................................XXXI

Capítulo 1. Introducción........................................................................................................... 1

1.1. Motivación y objetivos de la Tesis Doctoral ...................................................... 2

1.2. Contexto tecnológico ……………………………............................................. 3

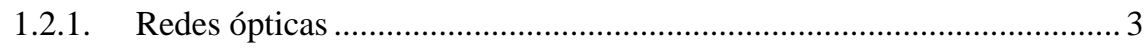

1.2.2. Sistemas de radiocomunicaciones..................................................... 5

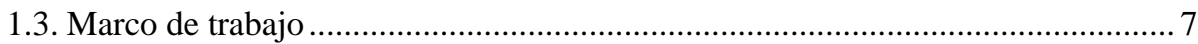

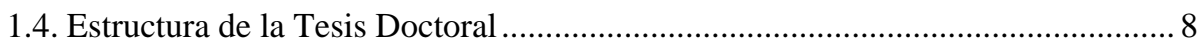

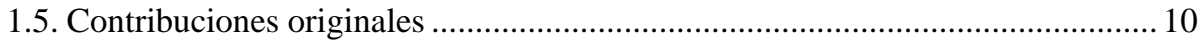

1.5.1. Contribuciones originales relacionadas con la Tesis Doctoral............... 10

1.5.2. Otras contribuciones originales .......................................................... 13 
Capítulo 2. Integración de transmisiones radio en redes ópticas de acceso............. 15

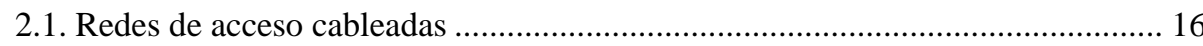

2.2. Redes de usuario vía radiofrecuencia ......................................................... 23

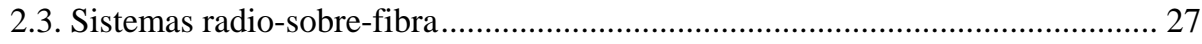

2.3.1. Multiplexación por división en subportadoras .................................... 29

2.3.2. Multiplexación por división en longitudes de onda.............................. 31

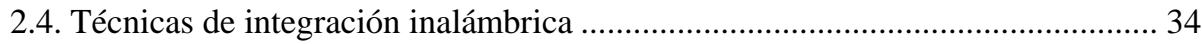

2.4.1. Sistemas pico-celulares basados en radio-sobre-fibra ........................ 35

2.5. Tecnologías radiofrecuencia disponibles comercialmente ............................. 37

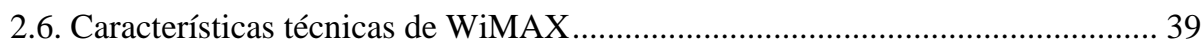

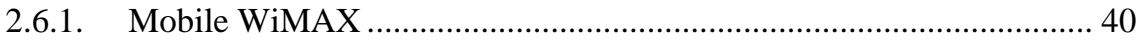

2.7. Características técnicas de UWB................................................................ 43

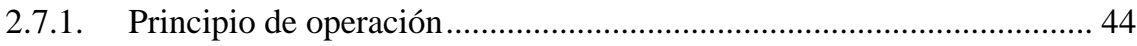

2.7.2. Sistemas basados en impulse-radio ................................................ 47

2.7.3. Sistemas basados en multi-band OFDM ........................................... 48

2.7.3.1. Especificaciones radio MB-OFDM UWB........................... 50

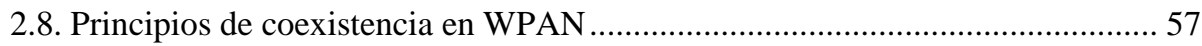

2.8.1. Técnica de mitigación por reducción del ciclo de trabajo .................... 59

2.8.2. Técnica de mitigación por detección y exclusión................................. 60

Capítulo 3. Coexistencia radio en entornos de redes personales ............................. 67

3.1. Coexistencia entre MB-OFDM UWB y WiMAX 802.16e ............................... 68

3.1.1. Modelo estándar del canal UWB ...................................................... 70

3.2. Análisis experimental y márgenes de protección en la coexistencia radio ......... 71

3.2.1. Escenario de medida experimental ............................................... 73

3.2.2. Prestaciones enlace WiMAX 802.16e en presencia de MB-OFDM UWB 78

3.2.3. Prestaciones enlace MB-OFDM UWB en presencia de WiMAX 802.16e 83

3.2.4. Prestaciones enlace MB-OFDM UWB con diferentes máscaras espectrales de potencia en presencia de WiMAX 802.16e 


\section{Capítulo 4. Coexistencia e integración en redes de acceso basadas en}

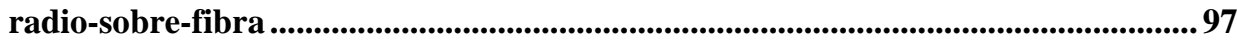

4.1. Componentes básicos en un sistema radio-sobre-fibra ..................................... 98

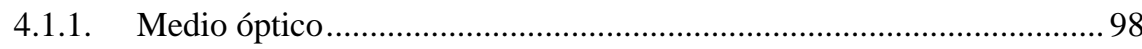

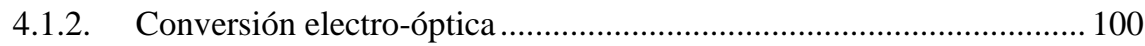

4.1.3. Conversión opto-electrónica ........................................................... 101

4.1.4. Integración del acceso inalámbrico.................................................... 102

4.2. Análisis experimental de la coexistencia UWB y WiMAX en sistemas

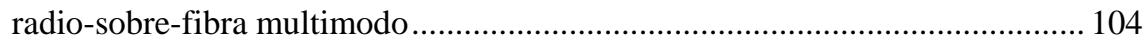

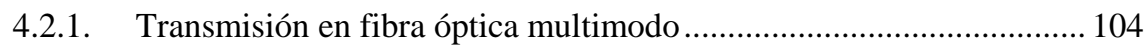

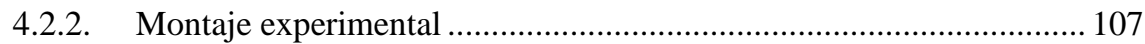

4.2.3. Evaluación experimental prestaciones coexistencia inalámbrica......... 110

4.3. Análisis experimental de la coexistencia UWB y WiMAX en sistemas radio-sobre-fibra estándar monomodo .............................................................. 115

4.3.1. Distribución UWB mediante sistemas RoSSMF .............................. 115

4.3.2. Prestaciones de la distribución MB-OFDM UWB en RoSSMF ......... 117

4.3.3. Prestaciones de la distribución en coexistencia RoSSMF sobre PON 124

4.3.3.1. Técnica PDM para la distribución simultánea ...................... 132

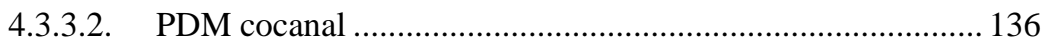

4.3.3.3. PDM de canal adyacente ................................................... 140

4.3.3.4. Prestaciones PDM para la distribución MB-OFDM UWB en modo dual ....................................................................... 143

\section{Capítulo 5. Linealización del modulador electro-óptico Mach-Zehnder en sistemas}

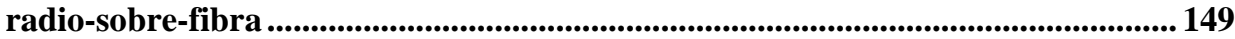

5.1. Modelo básico de un modulador electro-óptico Mach-Zehnder ....................... 150

5.1.1. Parámetros característicos........................................................... 151

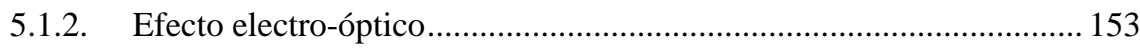

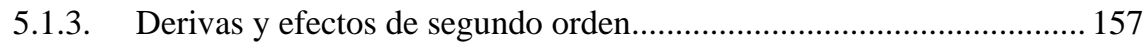

5.1.3.1. Deriva de la tensión de polarización.................................... 157

5.2. Técnicas de linealización de moduladores externos ...................................... 158 
5.2.1. Linealización eléctrica y mediante post-procesado .......................... 158

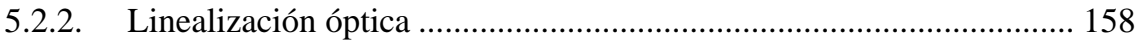

5.2.2.1. Configuración en cascada o serie ...................................... 159

5.2.2.2. Configuración en paralelo .................................................. 162

5.3. Análisis de técnica de linealización óptica dual en paralelo MZM .................. 167

5.3.1. Evaluación analítica y mediante simulación..................................... 169

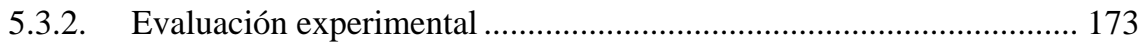

5.3.3. Derivas y efectos de segundo orden ........................................... 175

5.3.3.1. Derivas en la alimentación asimétrica ................................ 175

5.3.3.2. Derivas en la tensión de polarización.................................. 178

5.3.3.3. Deriva temporal.......................................................... 181

Capítulo 6. Conclusiones y líneas futuras ......................................................... 185

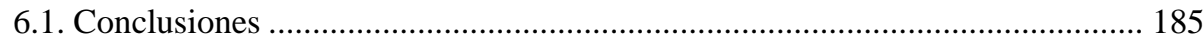

6.1.1. Coexistencia radio de WiMAX y UWB ........................................ 186

6.1.2. Distribución simultánea de WiMAX y UWB en sistemas RoF.......... 187

6.1.3. Linealización óptica de MZM para la distribución conjunta de WiMAX y UWB ..................................................................... 189

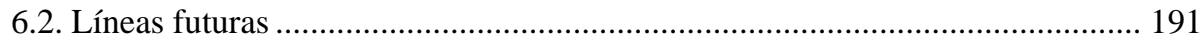

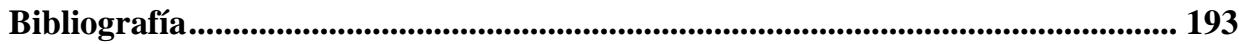




\section{Índice de figuras}

Figura 1.1. Concepto de redes ópticas de comunicaciones según su ámbito

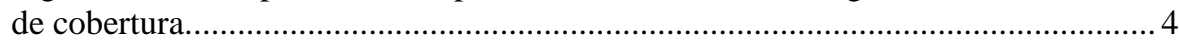

Figura 1.2. Estado del arte de las tecnologías de comunicaciones inalámbricas....... 6

Figura 2.1. Esquemas de distribución FTTx y nomenclatura................................... 18

Figura 2.2.Tasa de penetración de la tecnología FTTH por número de hogares conectados en Europa, Octubre 2008 (Fuente: FTTH Council Europe).

Figura 2.3.Tecnologías de comunicaciones vía radio según área de aplicación,

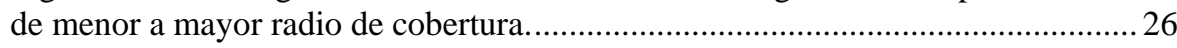

Figura 2.4. Arquitectura y escenario de aplicación de un sistema RoF..................... 28

Figura 2.5. Concepto de sistema RoF con transmisión basada en SCM. ................ 29

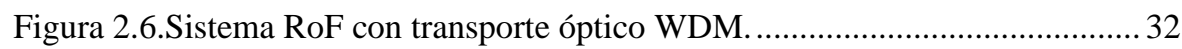

Figura 2.7.Arquitectura de una red de usuario basada en sistemas RoF con

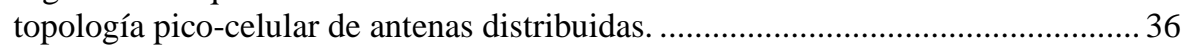

Figura 2.8. Frecuencias de trabajo y potencia transmitida de diversas

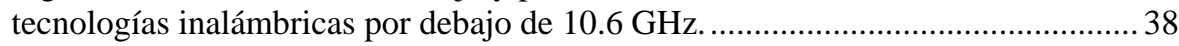

Figura 2.9. Estructura de trama TDD para Mobile WiMAX [IEEE80216e]............ 42

Figura 2.10. Máscara espectral de máxima EIRP para UWB radio según las

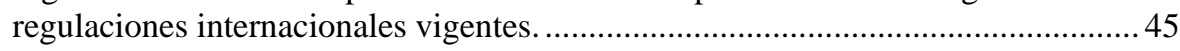

Figura 2.11. Implementaciones de la tecnología UWB.......................................... 47

Figura 2.12. Plataforma MB-OFDM UWB radio y sus aplicaciones actuales y futuras. PAL: Protocol Adaptation Layer, capa para la adaptación de

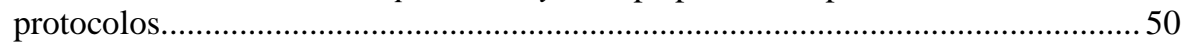

Figura 2.13. Plan de frecuencias MB-OFDM UWB............................................. 51

Figura 2.14. Transmisión tiempo-frecuencia de símbolos OFDM en MB-OFDM UWB con TFC1 en BG1. 
Figura 2.15. Configuración de subportadoras OFDM para MB-OFDM UWB..... 54

Figura 2.16. Estado actual de las regulaciones MB-OFDM UWB a nivel mundial, según WiMedia Alliance en Enero de 2009 [Wim]. 56

Figura 2.17. Dispositivos Mobile WiMAX (a) módem USB Motorola wi4 WiMAX USBw 100 [MOT09], (b) dispositivo Mobile WiMAX BreezeMAX Si de Alvarion Ltd. [ALV09b], y de dispositivos MB-OFDM UWB, (c) dispositivos Wireless USB Adapter Set de Olidata S.p.A. [OLI09], (d) televisión WOOO LCD HITACHI con Wireless HDMI [HIT09].. 58

Figura 3.1. Escenario conceptual de coexistencia radio entre UWB y WiMAX.... 69 Figura 3.2. Sala de reuniones de la Universidad Politécnica de Valencia utilizada para la evaluación experimental de la coexistencia radio entre UWB y WiMAX

Figura 3.3. Definición de EVM en base a (a) representación grafica y a el

(b) cálculo en función del símbolo recibido $Z_{n}$ y el símbolo de referencia $S_{n} \ldots \ldots . .72$

Figura 3.4. (a) Situación de la sala de reuniones. (b) Distribución y localización de los equipos usados en el estudio de coexistencia. La marca $\langle A\rangle$ se usa como referencia entre el plano real y la distribución recreada.

Figura 3.5. Distancias de los enlaces UWB y WiMAX y situación de las zonas 1, 2, 3 para técnicas de mitigación de la interferencia basadas en DAA.

Figura 3.6. Espectro de la señal UWB generada para una configuración tipo (a)

TFC1 y (b) TFC5 de $200 \mathrm{Mbit} / \mathrm{s} . \mathrm{RBW}=1 \mathrm{MHz}$ 76

Figura 3.7. Espectros de la señal WiMAX 802.16e generada para un ancho de banda nominal de (a) $5 \mathrm{MHz}$, (b) $10 \mathrm{MHz}$ y (c) $20 \mathrm{MHz}$. RBW $=1 \mathrm{MHz}$ 77

Figura 3.8. Montaje experimental para el estudio del enlace WiMAX 802.16e en presencia de un interferente MB-OFDM UWB. 78

Figura 3.9. Espectros de la señal WiMAX 802.16e recibida para un ancho de banda nominal de $10 \mathrm{MHz}$ y d $\mathrm{d}_{\text {WiMAX }}=5 \mathrm{~m}$ en presencia de un interferente MB-OFDM UWB TFC1 situado en (a) $d_{U W B}=0.10 \mathrm{~m} \mathrm{y} \mathrm{(b)} d_{U W B}=1 \mathrm{~m}$. $\mathrm{RBW}=1 \mathrm{MHz}$

Figura 3.10. EVM medido para un enlace WiMAX $5 \mathrm{MHz}, 10 \mathrm{MHz}$ y $20 \mathrm{MHz}$ BW con $\mathrm{d}_{\text {WiMAX }}=0.36 \mathrm{~m}$ ante la presencia de MB-OFDM UWB (a) TFC1, (b) TFC5 53.3 Mbit/s, y para MB-OFDM UWB (c) TFC1 y (d) TFC5 200 Mbit/s..... 80 Figura 3.11. EVM medido para un enlace WiMAX $5 \mathrm{MHz}, 10 \mathrm{MHz}$ y $20 \mathrm{MHz}$ $\mathrm{BW}$ con $\mathrm{d}_{\text {WiMAX }}=3 \mathrm{~m}$ ante la presencia de MB-OFDM UWB (a) TFC1 y (b) TFC5 $200 \mathrm{Mbit} / \mathrm{s}$.

Figura 3.12. EVM medido para un enlace WiMAX de $5 \mathrm{MHz}, 10 \mathrm{MHz}$ y $20 \mathrm{MHz}$ BW con $\mathrm{d}_{\mathrm{WiMAX}}=8 \mathrm{~m}$ ante la presencia de MB-OFDM UWB (a) TFC1 y (b) TFC5 $200 \mathrm{Mbit} / \mathrm{s}$ 81

Figura 3.13. EVM medido para WiMAX (a) $5 \mathrm{MHz}$, (b) $10 \mathrm{MHz}$ y (c) $20 \mathrm{MHz}$ BW en presencia de MB-OFDM UWB TFC5 $200 \mathrm{Mbit} / \mathrm{s}$. 82

Figura 3.14. Montaje experimental para el estudio del enlace MB-OFDM UWB en coexistencia con WiMAX. 
Figura 3.15. Espectros recibidos MB-OFDM UWB (a) TFC1 y (b) TFC5 $53.3 \mathrm{Mbit} / \mathrm{s}$ para $\mathrm{d}_{\mathrm{UWB}}=1 \mathrm{~m}$ ante la presencia de un interferente WiMAX $10 \mathrm{MHz}$ BW situado a $\mathrm{d}_{\text {WiMAX }}=5 \mathrm{~m}$. RBW $=1 \mathrm{MHz}$. 84

Figura 3.16. EVM medido para un enlace MB-OFDM UWB (a) TFC1 y (b) TFC $553.3 \mathrm{Mbit} / \mathrm{s}$, y (c) TFC1, (d) TFC $5200 \mathrm{Mbit} / \mathrm{s}$ con $\mathrm{d}_{\mathrm{UwB}}=0.5 \mathrm{~m}$ para diferentes configuraciones WiMAX interferente. 85

Figura 3.17. EVM medido para un enlace MB-OFDM UWB (a) TFC1 y (b) TFC $553.3 \mathrm{Mbit} / \mathrm{s}$, y (c) TFC1, (d) TFC $5200 \mathrm{Mbit} / \mathrm{s}$ con $\mathrm{d}_{\mathrm{UWB}}=1 \mathrm{~m}$ para diferentes configuraciones WiMAX interferente.

Figura 3.18. EVM medido para un enlace MB-OFDM UWB (a) TFC1 y (b) TFC $553.3 \mathrm{Mbit} / \mathrm{s}, \mathrm{y}$ (c) TFC1, (d) TFC $5200 \mathrm{Mbit} / \mathrm{s}$ con $\mathrm{d}_{\mathrm{UwB}}=2 \mathrm{~m}$ para diferentes configuraciones WiMAX interferente.

Figura 3.19. EVM medido para un enlace MB-OFDM UWB (a) TFC1 y (b) TFC $5200 \mathrm{Mbit} / \mathrm{s}$ en presencia de interferente WiMAX $10 \mathrm{MHz}$ BW. 88

Figura 3.20. EVM medido por subportadora MB-OFDM UWB TFC1

53.3 Mbit/s (a) sin WiMAX, (b) con WiMAX 5 MHz BW sito en $\mathrm{d}_{\text {WiMAX }}=1 \mathrm{~m}$; y para MB-OFDM UWB TFC5 $53.3 \mathrm{Mbit} / \mathrm{s}$ (c) sin WiMAX y con (d) WiMAX $5 \mathrm{MHz}$ BW sito en $\mathrm{d}_{\text {WiMAX }}=1 \mathrm{~m}$.

Figura 3.21. EVM medido por subportadora MB-OFDM UWB TFC1 $200 \mathrm{Mbit} / \mathrm{s}$ (a) sin WiMAX, (b) con WiMAX $5 \mathrm{MHz}$ BW sito en $\mathrm{d}_{\text {WiMAX }}=1 \mathrm{~m}$; para MB-OFDM UWB TFC1 $53.3 \mathrm{Mbit} / \mathrm{s}$ con (c) WiMAX $10 \mathrm{MHz}$ BW sito en $\mathrm{d}_{\text {WiMAX }}=1 \mathrm{~m}$ y (d) WiMAX $5 \mathrm{MHz} \mathrm{BW}$ sito en $\mathrm{d}_{\text {WiMAX }}=3 \mathrm{~m}$. 90

Figura 3.22. Montaje experimental para el estudio del enlace MB-OFDM UWB con variaciones de su EIRP máxima. 92

Figura 3.23. EVM medido para un enlace MB-OFDM UWB (a) TFC1 y (b)

TFC5 53.3 Mbit/s en presencia de WiMAX, con EIRP según [ECMA368].

Figura 3.24. EVM medido para enlace MB-OFDM UWB (a) TFC1 y (b) TFC5 53.3 Mbit/s en presencia de WiMAX, con EIRP máxima de $-35 \mathrm{dBm} / \mathrm{MHz}$ 93

Figura 3.25. EVM medido para un enlace MB-OFDM UWB (a) TFC1, (b)

TFC5 53.3 Mbit/s en presencia de WiMAX, con EIRP máxima de $-21 \mathrm{dBm} / \mathrm{MHz}$. 94

Figura 3.26. EVM medido por subportadora MB-OFDM UWB TFC1

53.3 Mbit/s con EIRP (a) $-41.3 \mathrm{dBm} / \mathrm{MHz}$ (b) $-35 \mathrm{dBm} / \mathrm{MHz}$ y (c) $-21 \mathrm{dBm} / \mathrm{MHz}$, ante presencia interferente WiMAX $5 \mathrm{MHz} \mathrm{BW}$. 94

Figura 4.1. Componentes básicos de un sistema RoF. 98

Figura 4.2. Representación de distribución multiservicio en entornos interiores basada en redes con transmisión RoF. 102

Figura 4.3. Ejemplos de aplicación de sistemas RoMMF en la distribución de UWB para la extensión del acceso mediante (a) la segmentación celular en edificios y (b) la conexión entre áreas dispersas [Ran07]. 106

Figura 4.4. Montaje experimental para la evaluación de la coexistencia inalámbrica mediante un sistema RoMMF. 
Figura 4.5. EVM medido para diferentes potencias RF en VCSEL tras la transmisión sobre MMF de la señal (a) WiMAX 802.16d y (b)

MB-OFDM UWB.

Figura 4.6. Densidad espectral de potencia de la señal conjunta UWB y

WiMAX (a) antes del VCSEL y tras la transmisión óptica sobre (b) 400 m de

MMF. RBW = $1 \mathrm{MHz}$.

Figura 4.7. EVM medido para trasmisiones sobre diferentes longitudes MMF de (a) MB-OFDM UWB y (b) WiMAX 802.16d en el sistema propuesto.

Figura 4.8. EVM medido en el receptor UWB tras el enlace radio para la transmisión en (a) RoMMF de MB-OFDM UWB aislado y la transmisión conjunta en (b) RoMMF de MB-OFDM UWB y WiMAX.

Figura 4.9. EVM medido en el receptor WiMAX tras el enlace radio para una transmisión conjunta de MB-OFDM UWB y WiMAX mediante un sistema RoMMF.

Figura 4.10. Esquema conceptual para la distribución de MB-OFDM UWB en redes FTTH mediante sistemas RoF

Figura 4.11. Esquema del montaje experimental para la evaluación de la

distribución de MB-OFDM UWB en un sistema RoSSMF con enlaces FTTH. .. 118

Figura 4.12. Densidad espectral de potencia de la señal MB-OFDM UWB (a) antes de su modulación óptica en el MZM y (b) tras su transmisión sobre un enlace de $25 \mathrm{~km}$ de fibra SSMF. RBW = $1 \mathrm{MHz}$.

Figura 4.13. EVM UWB medido para diferentes distancias SSMF y potencias ópticas del EDFA (CO) para (a) Ch1 y (b) Ch2.

Figura 4.14. (a) Espectro de la señal MB-OFDM UWB recibida tras un enlace de $25 \mathrm{~km}$ SSMF con $1.5 \mathrm{~m}$ de propagación radio. $\mathrm{RBW}=1 \mathrm{MHz}$.

Constelaciones asociadas a MB-OFDM UWB (b) Ch1 y (c) Ch2 para una transmisión de $25 \mathrm{~km}$ SSMF sin enlace radio. Constelaciones asociadas a MB-OFDM UWB (d) Ch1 y (e) Ch2 tras un enlace $25 \mathrm{~km}$ SSMF con $1.5 \mathrm{~m}$ de propagación radio.

Figura 4.15. EVM UWB medido según diferentes distancias de propagación radio para cada enlace SSMF para una potencia óptica entregada de -34.31 dBm en foto-detección para MB-OFDM UWB (a) Ch1 y (b) Ch2.

Figura 4.16. EVM UWB medido según diferentes enlaces SSMF para diferentes distancias de propagación radio con una potencia óptica entregada de -34.31 dBm en foto-detección para MB-OFDM UWB (a) Ch1 y (b) Ch2.

Figura 4.17. Concepto de la distribución simultánea de servicios inalámbricos basados en WiMAX y UWB mediante el uso de sistemas RoSSMF en redes PON.

Figura 4.18. Esquema del montaje experimental realizado para la evaluación de la distribución simultánea de WiMAX y UWB en una red PON mediante un sistema RoSSMF.

Figura 4.19. Densidad espectral de potencia de la transmisión aislada (a) de la señal MB-OFDM UWB Ch1 y Ch2, y (b) de la señal WiMAX 20 MHz BW en una red $\mathrm{PON}$ bajo configuración $\mathrm{B} 2 \mathrm{~B}$. $\mathrm{RBW}=1 \mathrm{MHz}$. 
Figura 4.20. EVM medido para la distribución aislada de MB-OFDM UWB (a) $\mathrm{Ch} 1$ y (b) Ch2 para diferentes potencias ópticas entregadas a la red PON propuesta sobre cada enlace SSMF bajo estudio.

Figura 4.21. EVM medido para la distribución aislada de las diferentes configuraciones WiMAX 802.16e para enlaces SSMF de (a) $10 \mathrm{~km}$ y (b) $25 \mathrm{~km}$ para diferentes potencias ópticas entregadas a la red PON propuesta

Figura 4.22. EVM UWB medido para una distribución conjunta de MB-OFDM UWB y WiMAX 802.16e en la red PON propuesta para las configuraciones: UWB Ch1 con WiMAX (a) $5 \mathrm{MHz}$ (b) $10 \mathrm{MHz}$ y (c) $20 \mathrm{MHz}$ BW, UWB Ch2 con WiMAX (d) $5 \mathrm{MHz}$, (e) $10 \mathrm{MHz}$ y (f) $20 \mathrm{MHz} \mathrm{BW}$, y por último UWB TFC1 con WiMAX (g) $5 \mathrm{MHz}$ (h) $10 \mathrm{MHz}$ y (i) $20 \mathrm{MHz} \mathrm{BW}$....... 130 Figura 4.23. EVM WiMAX medido para una distribución conjunta MB-OFDM UWB y WiMAX 802.16e en la red PON propuesta para las configuraciones: WiMAX $10 \mathrm{MHz}$ BW para (a) UWB Ch1 y Ch2, y para (b) UWB TFC1.

Figura 4.24. Concepto de convergencia inalámbrica de servicios radio WiMAX y UWB sobre una red PON con RoF mediante técnicas PDM.

Figura 4.25. (a) Representación de las polarizaciones ortogonales de la señal multiplexada en polarización (PM) entregada a la red PON. Esquemas de multiplexación por división en polarización de tipo (b) cocanal, (c) de canal adyacente y (d) UWB dual multiplexado.

Figura 4.26. Esquema del montaje experimental para la evaluación de la distribución simultánea de señales UWB y WiMAX en sistemas RoF PON mediante distintas estrategias PDM.

Figura 4.27. Densidad espectral de potencia de las señales MB-OFDM UWB y WiMAX 802.16e recibidas tras un enlace $25 \mathrm{~km}$ SSMF en la configuración PDM cocanal para (a) Pol. A y (b) Pol. B. RBW = $1 \mathrm{MHz}$.

Figura 4.28. EVM medido para las señales (a) UWB Ch1 y Ch2 con Pol. A y

(b) WiMAX $5 \mathrm{MHz}$ BW con Pol. B recibidas, según diferentes potencias ópticas entregadas en el sistema RoSSMF PON propuesto.

Figura 4.29. EVM medido para las señales (a) UWB Ch1 y Ch2 con Pol. A y

(b) WiMAX $10 \mathrm{MHz}$ BW con Pol. B recibidas, según diferentes potencias ópticas entregadas en el sistema RoSSMF PON propuesto.

Figura 4.30. EVM medido para las (a) UWB Ch1 y Ch2 con Pol. A y (b)

WiMAX $20 \mathrm{MHz}$ BW con Pol. B recibidas según diferentes potencias ópticas entregadas en el sistema RoSSMF PON propuesto.

Figura 4.31. Densidad espectral de potencia de las señales MB-OFDM UWB y WiMAX 802.16e recibidas tras un enlace de $25 \mathrm{~km}$ SSMF en la configuración PDM de canal adyacente para (a) Pol. A y (b) Pol. B. RBW $=1 \mathrm{MHz}$. 140

Figura 4.32. EVM medido para las señales (a) UWB Ch2 y Ch3 con Pol. A y (b) WiMAX $10 \mathrm{MHz}$ BW con Pol. B recibidas según diferentes potencias ópticas entregadas en el sistema RoSSMF PON propuesto. 
Figura 4.33. EVM medido para las señales recibidas WiMAX tras un enlace de $25 \mathrm{~km}$ SSMF según diferentes potencias ópticas entregadas en el sistema RoSSMF PON propuesto para el caso PDM (a) cocanal y (b) adyacente.

Figura 4.34. EVM medido para una transmisión aislada de señales

MB-OFDM UWB (a) Ch2 y (b) Ch3 según Pol. A en el sistema RoSSMF PON propuesto con PDM para diferentes longitudes de enlace SSMF.

Figura 4.35. Densidad espectral de potencia de las señales MB-OFDM UWB recibida tras $25 \mathrm{~km}$ SSMF en la configuración PDM para transmisión aislada de UWB Ch2 y Ch3 según Pol. A en (a) punto (5) y (b) punto (6) del montaje, y de UWB Ch2 y Ch 3 según Pol. B. en (c) punto (5) y (d) punto (6) del montaje RoSSMF PON propuesto. RBW $=1 \mathrm{MHz}$.

Figura 4.36. EVM UWB medido para las señales MB-OFDM UWB Ch2 y Ch3 (a) Pol. A y (b) Pol. B recibidas para diferentes potencias ópticas entregadas en el sistema RoSSMF PON con PDM propuesto.

Figura 5.1. Esquema del modulador electro-óptico de intensidad basado en interferómetro Mach-Zehnder.

Figura 5.2. Función de transferencia óptica para un MZM con $V_{\pi}(D C)=4.5 \mathrm{~V}$ con pérdidas.

Figura 5.3. Esquema de la propuesta dual serie MZM (DSMZM) para

linealización óptica en banda estrecha por [Bet94]. 160

Figura 5.4. Esquema de la propuesta dual serie MZM (DSMZM) para

linealización óptica en banda ancha por [Bri92].

Figura 5.5. Esquema de la propuesta serie MZM mediante acopladores para linealización óptica hasta quinto orden [Bur95].

Figura 5.6. Esquema de la propuesta de linealización óptica serie en banda ancha con 2 MZM y una estructura de acopladores [Wan92]. 161

Figura 5.7. Esquema de la propuesta de linealización óptica dual paralelo con 2 MZM (DPMZM) [Kor90].

Figura 5.8. Esquema de la propuesta de linealización óptica dual paralelo con 2 MZM (DPMZM) y modulación SSB. Diagrama de funciones Bessel de cada rama del MZM y de la señal óptica final tanto en campo como en RF [Shi01]. .. 163 Figura 5.9. Esquema de la propuesta de linealización óptica dual paralelo con 2 MZM (DPMZM) y modulación SSB [Kaw03].

Figura 5.10. Resumen de técnicas de linealización ópticas basadas en moduladores y acopladores direccionales serie y paralelo, expuestas en [Cum98]. 166

Figura 5.11. Concepto de linealización óptica DPMZM con alimentación óptica y RF asimétrica y foto-detección diferencial. 168

Figura 5.12. Valores analíticos de IMD3 para el esquema de linealización óptica DPMZM propuesto, con $\mathrm{f}_{1}=1 \mathrm{GHz}$ y $\mathrm{f}_{2}=1.1 \mathrm{GHz}$.

Figura 5.13. Valores analíticos de $\mathrm{H}_{0}$ para el esquema de linealización óptica DPMZM propuesto, con $\mathrm{f}_{1}=1 \mathrm{GHz}$ y $\mathrm{f}_{2}=1.1 \mathrm{GHz}$. 
Figura 5.14. Esquema del montaje experimental realizado para evaluar la técnica de linealización óptica DPMZM con alimentación asimétrica.

Figura 5.15. Resultados HD3 medidos para diferentes atenuaciones ópticas según una atenuación RF fija.

Figura 5.16. Comparativa de resultados (a) HD3 y (b) potencia de portadora $\mathrm{H}_{0}$ en simulación óptica y de forma experimental para una atenuación de RF fija de $6 \mathrm{~dB}$, con $f_{R F}=5 \mathrm{GHz}$.

Figura 5.17. Resultados simulados de rechazo de IMD3 en la arquitectura de linealización óptica DPMZM propuesta, para retardos en una de las ramas de alimentación RF, con alimentación asimétrica basada en $6 \mathrm{~dB}$ de atenuación óptica y $8 \mathrm{~dB}$ de atenuación eléctrica.

Figura 5.18. Resultados simulados de IMD3 en la arquitectura de linealización óptica DPMZM propuesta, para retardos en una de las ramas de alimentación óptica, con alimentación asimétrica basada en $6 \mathrm{~dB}$ de atenuación óptica y $8 \mathrm{~dB}$ de atenuación eléctrica.

Figura 5.19. (a) Rechazo de IMD3 e (b) IMD2 en la arquitectura de linealización óptica DPMZM propuesta, para variaciones simuladas de la tensión de polarización.

Figura 5.20. (a) Rechazo de IMD3 e (b) IMD2 en la arquitectura de linealización óptica DPMZM propuesta, para las variaciones simuladas de tensión de polarización mostradas en la Tabla 5.4. 



\section{Índice de tablas}

Tabla 2.1. Régimen binario por usuario y alcance máximo en distintas

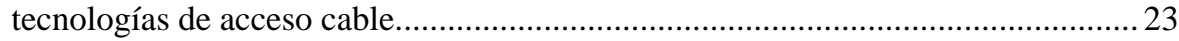

Tabla 2.2. Ventajas de los sistemas RoF en las redes de acceso ............................ 33

Tabla 2.3. Tecnologías de comunicaciones inalámbricas en entornos de corto y medio alcance

Tabla 2.4. Parámetros OFDM escalable para IEEE 802.16e de $5 \mathrm{~ms}$ de duración con CP de $1 / 8$....................................................................................... 42

Tabla 2.5. Plan detallado de frecuencias en MB-OFDM UWB ............................. 51

Tabla 2.6. Canalización MB-OFDM según códigos TFC para BG1 …..................52

Tabla 2.7. Canalización MB-OFDM según códigos TFC para BG5 ….................. 52

Tabla 2.8. Parámetros temporales de la señal MB-OFDM básica .......................... 53

Tabla 2.9. Características funcionales de un paquete de señal

MB-OFDM UWB básica .................................................................................... 54

Tabla 2.10. Parámetros de modulación de la señal MB-OFDM básica .................. 55

Tabla 2.11. Parámetros para la implementación de la técnica de mitigación

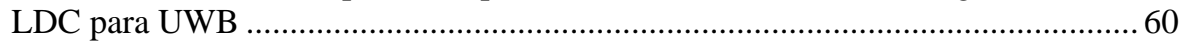

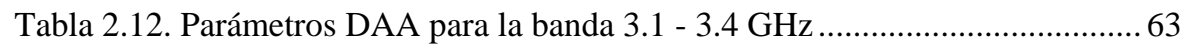

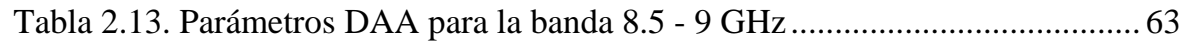

Tabla 2.14. Parámetros DAA para la banda 3.4 - $4.2 \mathrm{GHz}$..................................... 64

Tabla 2.15. Tiempo DAA según servicio BWA

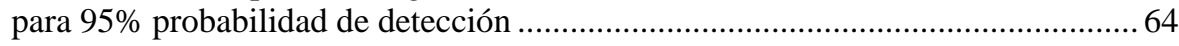

Tabla 3.1. Parámetros característicos modelo de canal UWB............................... 71

Tabla 3.2. Parámetros del enlace MB-OFDM UWB implementado...................... 75

Tabla 3.3. Parámetros del enlace WiMAX basado en IEEE 802. 16e .................... 77

Tabla 3.4. EVM medido para WiMAX sin presencia UWB ................................. 79

Tabla 3.5. EVM medido para MB-OFDM UWB sin presencia WiMAX............... 84 
Tabla 3.6. Márgenes interferente WiMAX 802.16e para comunicación MB-OFDM UWB. 95

Tabla 4.1. Parámetros de la señal WiMAX IEEE 802.16d generada .................... 108

Tabla 4.2. Parámetros de la señal MB-OFDM UWB generada 109

Tabla 4.3. Parámetros de la señal MB-OFDM UWB para el sistema RoSSMF bajo estudio

Tabla 4.4. EVM medido en el sistema MB-OFDM UWB propuesto sin transmisión óptica

Tabla 4.5. Parámetros MB-OFDM UWB para la distribución conjunta en una red PON

Tabla 4.6. Parámetros WiMAX IEEE 802. 16e para la distribución conjunta en una red $\mathrm{PON}$

Tabla 5.1. Comparativa prestaciones arquitecturas paralelo de linealización óptica en moduladores electro-ópticos

Tabla 5.2. Comparativa prestaciones arquitecturas serie de linealización óptica en moduladores electro-ópticos

Tabla 5.3. Valores HD3 obtenidos en simulación para factores de alimentación asimétrica óptimos en el esquema de linealización paralelo propuesto 172

Tabla 5.4. Valores de tensión de polarización para la simulación de la deriva en $\mathrm{V}_{\text {BIAS }}$ 


\section{Capítulo 1. Introducción}

Las redes y sistemas de telecomunicación actuales se enfrentan a la necesidad de proporcionar regímenes binarios cada vez más elevados a un mayor número de usuarios. Esta creciente necesidad se fundamenta, principalmente, en el aumento de tráfico de Internet y en particular en la introducción de la alta definición en servicios como el vídeo bajo demanda o los videojuegos en línea. A fin de satisfacer esta demanda, es necesario desarrollar nuevas técnicas para aumentar la capacidad de las redes ópticas de acceso conocidas como última milla (last mile), puesto que es la parte de la red con mayores limitaciones en la transmisión. En esta Tesis Doctoral se han considerado dos sistemas de comunicaciones inalámbricas capaces de proporcionar servicios con elevado régimen binario, en concreto WiMAX (Worldwide Interoperability for Microwave Access) [Nua07] y la tecnología de banda ultra-ancha UWB (Ultra-Wide Band) [Ben04]. Las redes ópticas consideradas en esta Tesis Doctoral son aquellas que se corresponden con las denominadas de fibra hasta el hogar (FTTH, Fibre-to-the-Home). La tecnología de transmisión óptica de las señales inalámbricas adoptada en esta Tesis Doctoral se realiza en su formato original, es decir, sin remodulaciones ni conversiones de frecuencia, en una o varias portadoras ópticas. Esta técnica de transmisión, denominada radio-sobre-fibra, (RoF, Radio-over-Fibre), es especialmente interesante en redes de acceso integradas. 


\subsection{Motivación y objetivos de la Tesis Doctoral}

La motivación de esta Tesis Doctoral se basa en primer lugar en la demanda de servicios de triple-play, cuádruple-play o quíntuple-play [Boh06]. En los cuales se distribuye el acceso de forma conjunta para servicios de telefonía, acceso a internet de alta velocidad, vídeo bajo demanda de alta definición y televisión de alta definición (HDTV, High-Definition Television) con contenidos distribuidos bajo demanda hacia el usuario final, que conforman el denominado triple-play [Far02]. Si en este modelo de distribución se incluyen servicios inalámbricos como WiMAX se conforma el denominado cuádruple-play [Ulm07]. Por otra parte, la agregación de servicios domóticos y de seguridad en el hogar del usuario engloban en el servicio quíntuple-play [Ulm07]. También cabe destacar la reciente introducción de servicios avanzados que requieren la transmisión de contenidos adicionales a través de Internet, tal como se recoge por ejemplo en la especificación Blu-Ray ${ }^{\mathrm{TM}} 2.0$ [BLU08]. El incremento de la capacidad de transmisión supone un reto tecnológico en las actuales redes ópticas de acceso debido a que es necesario proveer servicios a un gran número de usuarios mediante una infraestructura de red generalmente heterogénea [Far02].

En segundo lugar, los trabajos de investigación aquí presentados están motivados por el interés de proveer servicios en la red de acceso de manera integrada, es decir, realizando la transmisión primero en la red óptica de acceso y también una transmisión vía radiofrecuencia en el entorno del usuario [Kat09]. Se consideran las redes ópticas de acceso basadas en FTTH como las más adecuadas para alcanzar estos objetivos, puesto que no es necesaria una adaptación de la misma para la transmisión óptica de señales inalámbricas de elevado régimen binario como WiMAX y UWB. En el caso de considerar redes de acceso mixtas óptico eléctricas, como las basadas en fibra hasta el edificio (FTTB, Fibre-to-the-Building) o hasta el armario de distribución (FTTCab, Fibre-to-the-Cabinet) y con cable coaxial hasta el usuario final, sería necesario una adaptación de la parte de transmisión en la red de acceso. Por lo tanto, el uso de otras redes diferentes a FTTH supondría un aumento de costes y por lo tanto no sería interesante su utilización como redes de acceso integradas. Es necesario destacar que el uso de tecnologías RoF facilita la coexistencia, distribución conjunta de distintos servicios de manera centralizada y con menores costes de despliegue de red, por lo tanto haciéndola muy adecuada para la implementación de redes de acceso integradas.

El objetivo de esta Tesis Doctoral es el estudio de la integración y coexistencia inalámbrica en redes de acceso basadas en sistemas RoF, centrándose en el estudio de las tecnologías radio capaces de proveer servicios de elevado régimen binario como son UWB y WiMAX. Para ello, esta Tesis Doctoral desarrolla los siguientes objetivos:

- Estudio de la interferencia en entornos WPAN de sistemas de comunicaciones inalámbricas capaces de proveer elevados régimen binarios como UWB y 
WiMAX. Derivación de márgenes de seguridad para la coexistencia radio en entornos WPAN.

- Estudio e identificación de las limitaciones de la transmisión óptica de señales UWB y WiMAX en sistemas RoF con fibra monomodo y multimodo. Derivación de las limitaciones en el alcance y las prestaciones en la distribución conjunta de UWB y WiMAX en sistemas RoF.

- Propuesta y demostración experimental de técnicas de transmisión óptica para la mejora de la distribución simultánea de UWB y WiMAX en redes de acceso basadas en sistemas RoF.

- Estudio del impacto del modulador electro-óptico como dispositivo clave en la distribución y la convergencia inalámbrica UWB y WiMAX en sistemas RoF.

- Propuesta y demostración experimental de técnicas de linealización adecuadas para moduladores electro-ópticos Mach-Zehnder operando con tecnologías RoF.

\subsection{Contexto tecnológico}

\subsubsection{Redes ópticas}

Las redes de comunicaciones por fibra óptica se han desarrollado de forma exponencial desde la década de los ochenta, con la introducción de la fibra monomodo en los grandes enlaces de comunicaciones, en las denominadas redes de larga distancia. El uso de la fibra óptica como guía-onda de impulsos de luz en materiales no conductores como sustituto del par de cobre en enlaces de comunicaciones fue propuesto por primera vez en un artículo teórico en 1966 por el Dr. C. Kao y el Dr. G. Hockham de los laboratorios de Standard Telecommunications de Inglaterra [Kao66]. En el mismo artículo, los autores demuestran que las grandes pérdidas de transmisión de las fibras ópticas existentes se podían disminuir de $1000 \mathrm{~dB} / \mathrm{km}$ hasta $20 \mathrm{~dB} / \mathrm{km}$ depurando las impurezas (agua, aire) presentes en los cristales usados para la fabricación de las mismas. Con la disminución de las pérdidas en la fibra óptica, los sistemas de comunicaciones ópticos necesitarían amplificadores para la transmisión de la señal óptica en términos de kilómetros y no de metros, de forma que serían comparables a los repetidores telefónicos de los sistemas de par de cobre. El rápido desarrollo de los primeros láseres ópticos de semiconductor a temperatura ambiente por el Dr. D. Payne de la Universidad de Southampton y E. Desurvire de Bell Labs, junto con la fabricación de la primera fibra óptica de cientos de metros y $20 \mathrm{~dB} / \mathrm{km}$ de atenuación óptica desarrollada por Corning Glass Works en 1970, permitió trasladar los sistemas de comunicaciones ópticas de los laboratorios a la ingeniería aplicada. Los 
primeros sistemas de comunicaciones ópticas de fibra se basaron en fibra óptica multimodo debido a su método de fabricación [Des94]. En la década de los 80 se introduce la nueva generación de fibra monomodo, con núcleos de sílice puro que rebajan la atenuación hasta el límite teórico de $0.2 \mathrm{~dB} / \mathrm{km}$ de atenuación. La aparición de los amplificadores de fibra dopada en 1987 [Des87], [Mea87], permite la amplificación simultánea de múltiples longitudes de onda, reduciendo costes al eliminar las etapas de conversión óptica-eléctrica-óptica y de demultiplexación-multiplexación en cada etapa amplificadora. Estos sistemas facilitan el despliegue de enlaces ópticos transoceánicos y redes de transmisión óptica, duplicándose la capacidad de los mismos conforme avanza y madura la tecnología de comunicaciones ópticas.

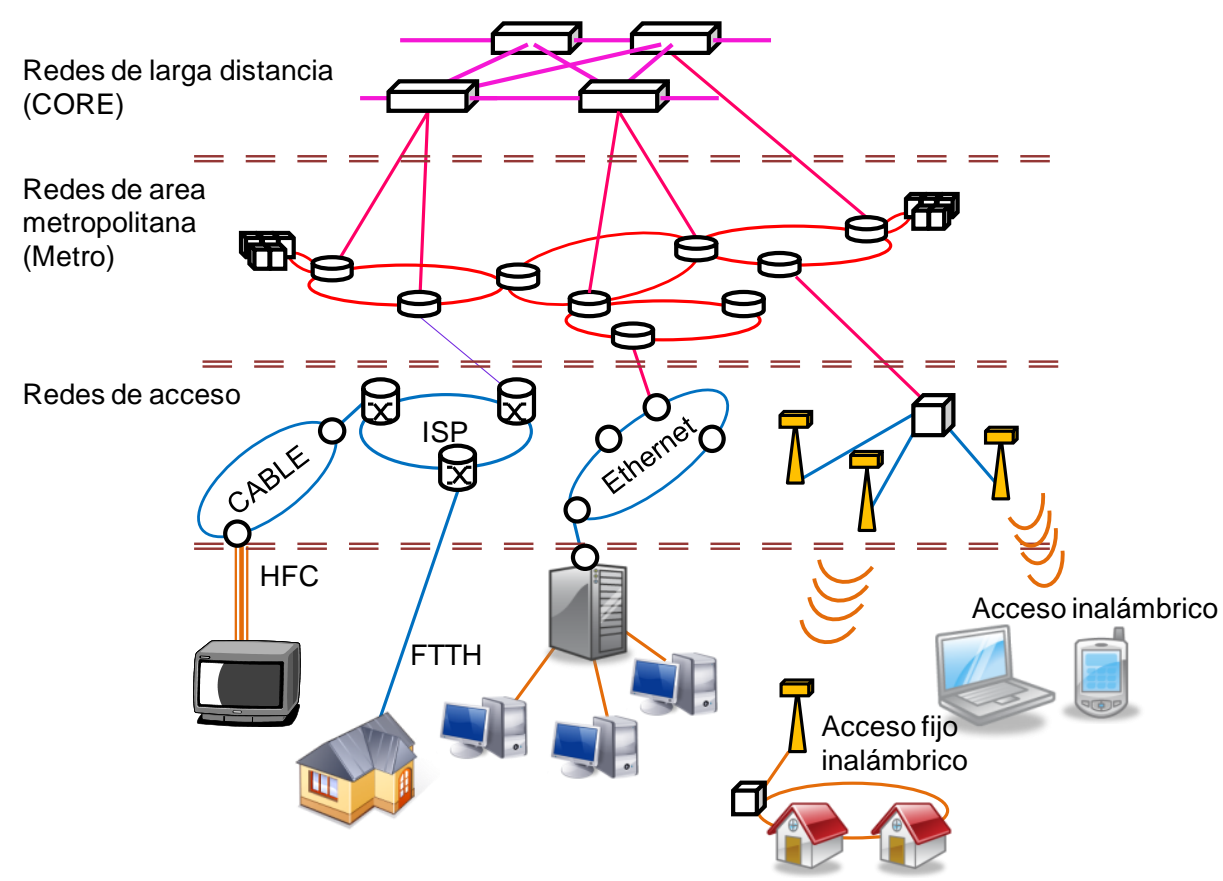

Figura 1.1. Concepto de redes ópticas de comunicaciones según su ámbito de cobertura.

Las actuales redes de comunicaciones ópticas se pueden clasificar según ámbitos de cobertura en redes de larga distancia, de media distancia o metropolitanas y de acceso [Ram98]. Las redes de larga distancia se caracterizan por conectar centrales de nivel regional o nacional. Los enlaces de comunicaciones en una red de larga distancia están interconectados mediante nodos internos o enrutadores (routers), formando el núcleo central o core de la red mundial y conectando entre sí diferentes redes metropolitanas. Su función es transportar gran cantidad de datos a través de largas distancias. Las redes metropolitanas son consideradas en ocasiones parte de la red troncal que enlaza los 
puntos de agrupación de tráfico metropolitano como centros de datos, empresas, universidades, etc. Tanto en los enlaces de comunicaciones de larga distancia (submarinos y terrestres) como en las redes metro, la fibra óptica desarrolla un papel fundamental. Por último, las redes de acceso permiten la conexión con los usuarios finales, haciendo de enlace entre los usuarios y las redes metropolitanas. Actualmente, en las redes de acceso se utilizan tecnologías como el par trenzado, conexiones vía satélite, la fibra óptica o el acceso inalámbrico. En la Figura 1.1 se pueden observar las diferentes redes de comunicaciones según criterios geográficos, desde las redes de comunicaciones ópticas de larga distancia hasta la red de acceso hacia el usuario final.

\subsubsection{Sistemas de radiocomunicaciones}

El rápido crecimiento de los servicios basados en transmisión de datos, la ubicuidad de las conexiones, la portabilidad de los terminales, y la demanda de servicios que provean elevados regímenes binarios de forma continua, han revolucionado las comunicaciones inalámbricas actuales. Esta demanda de servicios se puede valorar teniendo en cuenta ciertas magnitudes de las redes móviles actuales, como por ejemplo el número de líneas de comunicaciones móviles registradas por los operadores, más de 5 millones según datos de la comisión del mercado de las telecomunicaciones [CMT09], lo que supone 111.1 líneas de telefonía móvil por cada 100 habitantes en España. A nivel global existen unos 3950 millones de líneas de telefonía móvil suscritas, lo que indica una penetración mayor del $60 \%$ de la población mundial, según informes de la asociación de fabricantes de GSM (Global System for Mobile communications) [GSA09]. Este ratio varía según la zona geográfica, siendo del 10-15\% en el continente africano y mayor del $100 \%$ en Europa. Este número de líneas suscritas son, en la mayoría de casos, para uso de telefonía convencional o de segunda generación (2G), aunque se observa como la telefonía de tercera generación (3G) supone un 42,4\% del total. Las tecnologías 3G predominantes son, con un $89.5 \%$ WCDMA (WCDMA, Wideband Code Division Multiple Access) y HSPA (HSPA, High Speed Packet Access) y con un 9.5 \% CDMA (CDMA, Code Division Multiple Access). Dentro de HSPA se estima que el $72 \%$ de las conexiones son capaces de soportar transmisiones con regímenes binarios de $3.7 \mathrm{Mbit} / \mathrm{s}$. La implantación de nuevos estándares basados en WCDMA y 3GPP (Third Generation Partnership Project), como es el futuro LTE (Long Term Evolution), augura el desarrollo a corto plazo de nuevos servicios que provean elevados regímenes binarios en entornos de largo alcance (WWAN, Wireless Wide Area Networks). Este futuro desarrollo se reflejará tanto en las infraestructuras como en la implementación de dispositivos que faciliten el acceso a estos servicios. 


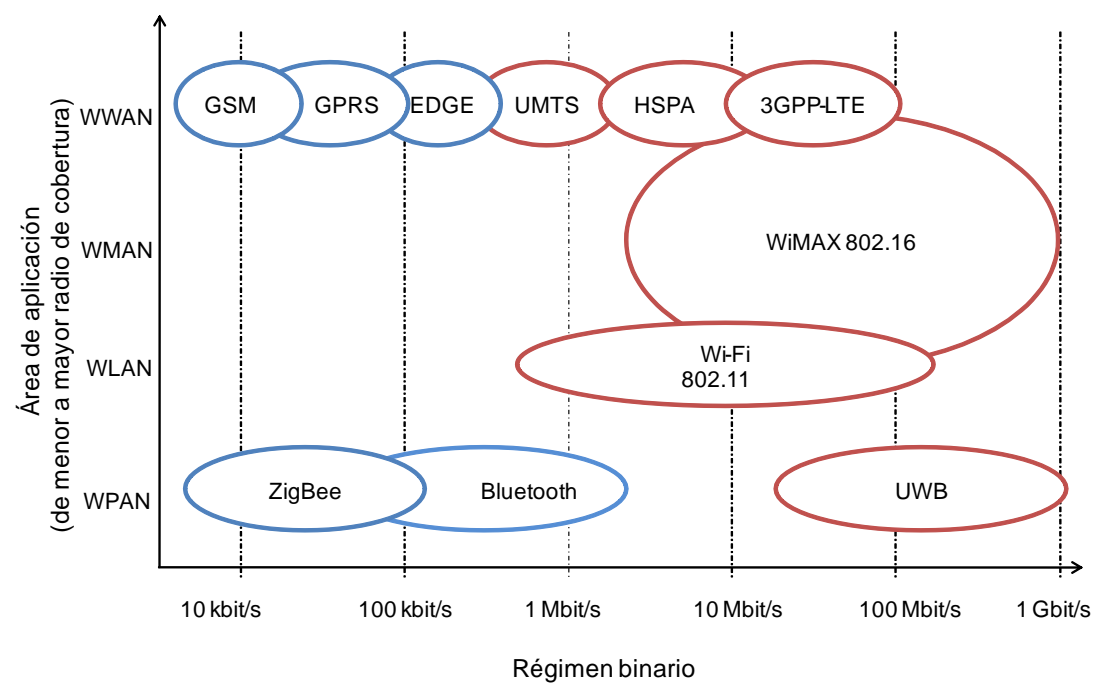

Figura 1.2. Estado del arte de las tecnologías de comunicaciones inalámbricas.

Analizando las comunicaciones inalámbricas que proporcionan servicios de elevado régimen binario en entornos de medio o corto alcance, se observa una gran diversidad tanto en su topología como en su funcionalidad. Desde el punto de vista de la topología existen tecnologías de comunicaciones inalámbricas basadas en sistemas multipunto entre una red de usuarios, como las redes Wi-Fi (Wireless Fidelity) basadas en la tecnologías IEEE 802.11, o punto-a-punto de alta velocidad para escenarios de corto alcance, como son las comunicaciones basadas en UWB. Desde el punto de vista funcional encontramos tecnologías que implementan transmisiones de datos a alta velocidad y en distancias muy cortas para sustituir los cables actuales, como es el USB inalámbrico (WUSB, Wireless Universal Serial Bus) basado en UWB, o que extienden los servicios móviles tradicionales en entornos de difícil cobertura, por ejemplo las redes WiMAX en entornos rurales.

En la Figura 1.2 se muestra el escenario actual en las redes de comunicaciones, donde se contemplan las diferentes tecnologías de radiocomunicaciones y su ámbito de aplicación, tales como redes inalámbricas personales (WPAN, Wireless Personal Area Networks), locales (WLAN, Wireless Local Area Networks), de tipo metropolitano (WMAN, Wireless Metropolitan Area Network) y de largo alcance (WWAN). Se observa la heterogeneidad de sistemas y ámbitos de aplicación, pero centrándonos en aquellas de elevado régimen binario, (>1 Gbit/s) actualmente existe una gran dinámica investigadora, industrial y social que está empujando su rápido desarrollo. Reflejo de estas actividades es la actual investigación en el estándar Bluetooth 3.0, que soporta conexiones inalámbricas a partir de $500 \mathrm{Mbit} / \mathrm{s}$, o los esfuerzos industriales en la 
implementación de la referencia USB inalámbrica para disminuir el impacto de los cables y evitar su uso en entornos domésticos.

Esta Tesis Doctoral presenta un estudio sobre las tecnologías de acceso inalámbricas UWB y WiMAX, como tecnologías de comunicaciones que proporcionan elevados regímenes binarios que comparten escenarios de aplicación como WPAN o WLAN. El estudio de la coexistencia de ambas tecnologías permitirá su distribución conjunta y rápido despliegue mediante el uso de sistemas RoF como red de acceso.

\subsection{Marco de trabajo}

Los trabajos de investigación presentados en esta Tesis Doctoral han sido desarrollados íntegramente en el Área de Sistemas y Redes del Instituto Universitario de Investigación de Tecnología Nanofotónica (VNTC, Valencia Nanophotonics Technology Center) [VNTC] de la Universidad Politécnica de Valencia. Esta Tesis Doctoral se enmarca en la línea de investigación de Redes Ópticas de Acceso del VNTC que, entre otras actividades de investigación, se centra en la distribución de señales de banda ultra-ancha y la mejora de los dispositivos ópticos y opto-electrónicos que permiten mejores prestaciones, mayor eficiencia $y$ menores costes de despliegue de la red.

La temática de esta Tesis Doctoral se ha alineado con los objetivos propuestos en los proyectos de investigación FREDIT [FREDIT] e ICT-UCELLS [UCELLS], en los cuales el VNTC ha participado durante los últimos 4 años. Una de las aportaciones del VNTC en el proyecto FREDIT ha sido el estudio e implementación de técnicas de linealización ópticas en dispositivos electro-ópticos para el aumento del margen dinámico de los receptores electrónicos digitales. El proyecto europeo UCELLS propone el concepto de clústeres de pico-celdas para la gestión de redes de comunicaciones UWB. Este proyecto contempla desarrollos tecnológicos en el ámbito de la coordinación entre dispositivos UWB, la distribución de señales UWB en RoF, la gestión de las redes de comunicaciones UWB, el desarrollo de estrategias de control y la gestión de las interferencias de otros sistemas de comunicaciones como WiMAX, Wi-Fi o UMTS en los escenarios de trabajo. El proyecto nacional FREDIT finalizó con éxito en el año 2008, y el proyecto europeo UCELLS se encuentra en desarrollo y concluirá a finales de 2010.

Otros proyectos y líneas de investigación como los sistemas ópticos integrados de bajo coste, los sistemas RoF y la convergencia y distribución de las actuales tecnologías radio, han influido claramente en el marco definitivo de esta Tesis Doctoral. También cabe destacar la participación en los proyectos europeos TOPRATE [TOPRATE] y IST-UROOF [UROOF] así como el apoyo recibido por parte de las redes de excelencia 
europeas E-photon/One+ [E-photon/One], ISIS [ISIS] y BONE [BONE] que han permitido difundir parte de los resultados de esta Tesis Doctoral en ámbitos internacionales.

\subsection{Estructura de la Tesis Doctoral}

Los principales contenidos de esta Tesis Doctoral se estructuran en 6 capítulos.

En el capítulo 1 se presenta una visión general del marco de la Tesis Doctoral y se enumeran las contribuciones originales derivadas de la misma. En el capítulo 2, se estudian las redes acceso vía cable y vía radio. Posteriormente, se plantean los requisitos para la convergencia inalámbrica de servicios basados en UWB y WiMAX en sistemas RoF para escenarios de corto alcance y elevado régimen binario. Por ejemplo, en escenarios de tipo WPAN en los cuales las redes de acceso basadas en sistemas RoF son de tipo pico-celular. A su vez, se exponen los aspectos tecnológicos más relevantes de UWB y WiMAX y se revisan los conceptos de coexistencia radio en entornos WPAN cuando se utilizan técnicas de mitigación de la interferencia como la detección y exclusión (DAA, Detect-and-Avoid), estas técnicas se consideran en futuras aplicaciones de tipo cognitive radio, para el adecuado despliegue de la tecnología UWB.

En el capítulo 3 se aborda la coexistencia de WiMAX y UWB en escenarios de redes inalámbricas personales. Para ello, se propone la evaluación experimental de la coexistencia inalámbrica de sistemas MB-OFDM UWB y WiMAX 802.16e en un escenario WPAN. Los resultados obtenidos proporcionan márgenes de seguridad para la interoperabilidad entre ambas tecnologías radio, mostrando cómo la futura implementación de técnicas de mitigación de la interferencia es necesaria para asegurar la coexistencia de ambas tecnologías y su futuro despliegue.

Posteriormente, el capítulo 4 abarca la evaluación de la distribución conjunta de UWB y WiMAX mediante redes basadas en sistemas RoF, que permite un acceso ubicuo a las comunicaciones por parte del usuario, disponer de redes de distribución transparentes al servicio inalámbrico transmitido y reducir costes. Se evalúan experimentalmente dos escenarios RoF para la distribución y coexistencia de UWB y WiMAX. En el primer escenario, se evalúa una red basada en un sistema RoF sobre fibra multimodo para la distribución conjunta de MB-OFDM UWB y WiMAX IEEE 802.16d. Por otro lado, en el segundo escenario se evalúa una red basada en un sistema RoF sobre fibra monomodo donde converge el acceso radio para MB-OFDM UWB y WiMAX IEEE 802.16e (Mobile WiMAX). Los resultados obtenidos proporcionan las limitaciones en sus prestaciones y el alcance máximo en el despliegue de las redes de acceso basadas en sistemas RoF asegurando la convergencia inalámbrica de ambas tecnologías. A su vez, se proponen y demuestran experimentalmente técnicas de multiplexación por división en polarización en la 
transmisión óptica cuyos resultados demuestran un aumento de la capacidad y el régimen binario agregado en los sistemas RoF, además de asegurar la distribución conjunta de UWB y WiMAX mediante sistemas RoF.

En el capítulo 5 se analiza la convergencia inalámbrica desde el punto de vista de los dispositivos clave en la transmisión RoF. En este caso, se estudia el modulador electro-óptico Mach-Zehnder como dispositivo clave que limita el margen dinámico en los sistemas RoF. Se presentan técnicas de linealización de carácter óptico del modulador Mach-Zehnder que permiten aumentar el margen dinámico del sistema RoF en el cual se implementan. Posteriormente, se propone y demuestra experimentalmente una arquitectura dual de moduladores Mach-Zehnder en paralelo como técnica de linealización óptica. Los resultados obtenidos indican un aumento del margen dinámico del modulador electro-óptico en presencia de señales de radiofrecuencia (RF, Radio-Frequency) de banda ancha.

Finalmente, en el capítulo 6 se presentan las conclusiones más importantes derivadas del trabajo realizado en esta Tesis Doctoral, así como las posibles líneas futuras planteadas tras la realización de este trabajo.

Nota: Las expresiones numéricas de esta Tesis Doctoral se han definido con el punto como separador de la parte entera de la parte decimal, de acuerdo a la acepción de punto para usos no lingüísticos realizada por la Real Academia Española de la Lengua [RAE]. 


\subsection{Contribuciones originales}

\subsubsection{Contribuciones originales relacionadas con la Tesis Doctoral}

A continuación se presenta un listado completo de las contribuciones científicas a las que ha dado lugar el trabajo desarrollado en esta Tesis Doctoral.

\subsubsection{Publicaciones en revistas internacionales}

- J. Perez, M. Morant, R. Llorente and J. Marti, "Joint Distribution of Polarization-Multiplexed UWB and WiMAX Radio in PON," IEEE Journal of Lightwave Technology, special issue on "Future Optical Networks" 2009, vol. 27, no. 12, pp. 1912-1919, Junio 2009.

- M. Morant, J. Perez, R. Llorente and J. Marti, "Combined Analysis of OFDM-UWB Transmission in Integrated Photonic-Wireless Optical Access Networks," IEEE Photonics Technology Letters, vol. 21, no 19, pp. 1378-1380, Octubre 2009.

- R. Llorente, T. Alves, M. Morant, M. Beltran, J. Perez, A. Cartaxo, and J. Marti, "Ultra-Wideband Radio Signals Distribution in FTTH Networks," IEEE Photonics Technology Letters, vol. 20, no. 11, pp. 945 - 947, Junio 2008.

\subsubsection{Contribuciones a congresos internacionales}

- J. Perez, M. Morant, M. Beltran, R. Llorente, "Performance of MB-OFDM UWB and WiMAX IEEE 802.16e Converged Radio-over-Fibre in PON," in Proc. IEEE International Topical Meeting on Microwave Photonics MWP09, Th4.41, Octubre 2009.

- M. Morant, J. Perez, M. Beltrán, R. Llorente, "Performance Evaluation of In-Building Radio-over- Fibre Distribution of Multi-Band OFDM UWB Signals," in Proc. IEEE International Topical Meeting on Microwave Photonics MWP09, Th4.13, Octubre 2009.

- J. Perez, M. Beltran, M. Morant, L. Cavallin, R. Llorente, "Protection Margins for Joint Operation of WiMAX 802.16e and WiMedia-defined UWB Radio in Personal Area Networks", in Proc. IEEE International Conference on Ultra-Wideband ICUWB09, pp. 723-727, Septiembre 2009. 
- $\quad$ M. Beltran, M. Morant, J. Perez, R. Llorente, "Performance Evaluation of OFDM and Impulse-Radio Ultra-Wideband over Fibre Distribution for In-Building Networks", in Proc. IEEE International Conference on Ultra-Wideband ICUWB09, pp. 348-352, Septiembre 2009.

- J. Perez, M. Morant, L. Cavallin, M. Beltran, R. Gaudino, R. Llorente, "Experimental analysis of WiMedia-defined UWB and WiMAX 802.16e coexistence in personal area networks," in Proc. ICT Mobile and Wireless Communications Summit ICT-Mobile Summit 2009, Junio 2009.

- J. Perez, M. Beltran, M. Morant, R. Llorente, A. Rahim-Biswas, R. Piesiewicz, M. Cotton, D. Fuehrer, B. Selva, I. Bucaille, S. Zeisberg, "Experimental Analysis of 3.5 GHz WiMAX 802.16e Interference in WiMedia-defined UWB Radio Transmissions," in Proc. IEEE Vehicular Technology Conference VTC09-Spring, pp. 1-5, Mayo 2009.

- M. Morant, J. Perez, R. Llorente, J. Martí, "Transmission of $1.2 \mathrm{Gbit} / \mathrm{s}$ Polarization-Multiplexed UWB Signals in PON with $0.76 \mathrm{Bit} / \mathrm{s} / \mathrm{Hz}$ Spectral Efficiency," in Proc. Optical Fibre Communication Conference OFC09, paper OTuJ6, pp. 1-3, Abril 2009.

- M. Morant, J. Perez, M. Beltran, R. Llorente, J. Marti, "Integrated performance analysis of UWB wireless optical transmission in FTTH networks," in Proc. Annual Meeting of the IEEE Lasers and Electro-Optics Society LEOSO8, pp. 87-88, Noviembre 2008.

- R. Alemany, J. Perez, R. Llorente, V. Polo, J. Martí, "Coexistence of WiMAX 802.16d and MB-OFDM UWB in Radio over Multi-mode Fibre Indoor Systems," in Proc. IEEE International Topical Meeting on Microwave Photonics MWP08, pp. 74-77, Septiembre 2008.

- $\quad$ M. Morant, M. Beltran, J. Perez, R. Llorente, "Impulse-Radio Ultra Wide-Band Signals Distribution in FTTH Networks," in Proc. ISIS 6th Workshop, Stockholm 2008.

- $\quad$ M. Beltran, M. Morant, J. Perez, and R. Llorente, "UWB wireless coexistence by fibre-based photonic ADC interference monitoring," in Proc. $6^{\text {th }}$ ISIS Workshop, Stockholm 2008

- $\quad$ R. Llorente, J. Perez, M. Beltrán, M. Morant, J. Martí, "UWB picocell clusters: Real-time interference monitoring," in Proc. ICT Mobile and Wireless Communications Summit ICT-Mobile Summit 2008, Mayo 2008. 
- R. Llorente; T. Alves; M. Morant; M. Beltran; J. Perez; A. Cartaxo; J. Marti, "Optical Distribution of OFDM and Impulse-Radio UWB in FTTH networks," in Proc. Optical Fibre Communication Conference OFC08, paper JWA109, Abril 2008.

- J. Perez; M. Beltrán; R. Llorente; J. Martí, "Performance Evaluation of the Parallel Mach-Zehnder Differential Linearization Architecture," in Proc. E-PhotonONe+ $/$ COST 291 Summer School 2007 "Advanced optical communications systems: from short range to long haul networks, ” Julio 2007.

\subsubsection{Contribuciones a congresos nacionales}

- R. Llorente, J. Perez, M. Beltran, J. Martí, "Convertidores analógico-digital fotónicos: Tecnología y Aplicaciones en Telecomunicación,” XVII Jornadas TELECOM I+D 2007, Mayo 2007.

\subsubsection{Informes técnicos}

- R. Llorente, J. Perez, M. Beltrán, M. Morant, J. Duplicy, D. Meshulam, "First report on the UWB technology evolution," ICT UCELLS Project Deliverable D7.2, Diciembre 2008.

- R. Llorente, M. Morant, J. Perez, M. Beltrán, T. Danon, S. Mantel, D. Schmertz, Y. Otiker, J. Duplicy, J. Puche, T.v. Waterschoot, V. Lenir, T. Alves, A. Cartaxo, J. Romme, N. Amiot, B. Uguen, Y. Lostanlen, R. Bañales, "Report on Cellular-UWB functionalities, real-time UWB spectrum monitoring functionalities. Photonic-ADC and Radio-over-Fibre architecture, suitable scenarios and technology state-of the-art," ICT UCELLS Project Deliverable D2.1, Octubre 2008.

- J. Perez, I.J. Niño, C. Calvo, R. Lluch, R. Llorente, "Project Presentation and Website set-up," ICT UCELLS Project Deliverable D7.1, Marzo 2008. 


\subsubsection{Otras contribuciones originales}

Durante el periodo de Tesis Doctoral también se han llevado a cabo otros trabajos, los cuales no han sido incluidos en esta Tesis. Este trabajo no incluido ha dado lugar a las siguientes contribuciones:

\subsubsection{Contribuciones a congresos internacionales}

- R. Alemany, J. Perez, R. Llorente, "UMTS radio-over-fiber pico-cell interconnection employing uncooled DFB lasers for multi-mode fibre modulation bandwidth enhancement," in Proc. Annual Meeting of the IEEE Lasers and Electro-Optics Society LEOSO9, paper ThK5, Octubre 2009.

- $\quad$ M. Beltrán, R. Sambaraju, A. La Porta, R. Llorente, J. Perez, "Photonic Generation and Detection of Impulse-Radio Ultra-Wideband Signals for Simultaneous Ranging and Communications", in Proc. IEEE International Conference on Ultra-Wideband ICUWB09, pp. 428-432, Septiembre 2009.

- $\quad$ M. Beltran, M. Morant, J. Perez, R. Llorente, J. Marti, "Photonic generation and frequency up-conversion of impulse-radio UWB signals," in Proc. Annual Meeting of the IEEE Lasers and Electro-Optics Society LEOSO8, pp.498-499, Noviembre 2008.

- M. Morant, R. Llorente, J. Perez, M. Beltran, J. Marti, T. Alves, A. Cartaxo, "Impact of Pilot Tone-assisted Equalization in Wimedia-defined OFDM-UWB signals Transmission in FTTH networks," in Proc. IEEE International Topical Meeting on Microwave Photonics MWP08, pp. 217-220, Septiembre 2008.

- $\quad$ R. Llorente, M. Beltran, J. Perez, N. Uehara, M.S. Khan, J. Marti, "Long-term and Short-term Spectral Stability Characterization of Supercontinuum Laser Sources," in Proc. Annual Meeting of the IEEE Lasers and Electro-Optics Society LEOS2006, pp. 685-686, Octubre 2006.

- $\quad$ R. Llorente; J. Perez; R. Clavero; J. Marti, "Linear crosstalk spectral analysis in DWDM networks by a real-time optical Fourier transformer," SPIE Opto-Ireland 2005: Opto-electronics, Photonics Devices and Optical Networks, in Proceedings of SPIE, volume 5825, pp. 474-481, Octubre 2005. 


\subsubsection{Contribuciones a congresos nacionales}

- J. Perez, R. Llorente; J. Martí, “Análisis espectral de la diafonía lineal en redes DWDM mediante transformadores ópticos de Fourier en tiempo real," $X X$ Simposium Nacional de la Unión Científica Internacional de Radio URSI 2005, Mayo 2005.

\subsubsection{Informes técnicos}

- $\quad$ M. Schmidt, M. Witte, F. Buchali, E. Corbel, A.I. Siahlo, A.T. Clausen, L.K. Oxenløwe, J. Seoane, R. Llorente, R. Clavero, J. Perez, "Report on dynamic dispersion compensation (GVD, PMD) and channel monitoring”, IST TOPRATE Project Deliverable D2.4, 2004

- $\quad$ M. Schmidt, M. Witte, F. Buchali, E. Corbel, A.I. Siahlo, A.T. Clausen, L.K. Oxenløwe, J. Seoane, R. Llorente, R. Clavero, J. Perez, "Public report on Terabit/s Transmission aspects within TOPRATE," IST TOPRATE Project Deliverable D2.5, 2004. 


\section{Capítulo 2. Integración de transmisiones radio en redes ópticas de acceso}

Tal y como se ha comentado en el capítulo anterior de esta Tesis Doctoral, existe una creciente demanda de mayores regímenes binarios, principalmente por parte de usuarios residenciales y de negocios [Kim09]. Esta demanda se puede satisfacer mediante dos estrategias: (i) la distribución conjunta o coexistencia de transmisiones radio en base a la tecnología inalámbrica adecuada a los servicios demandados, y (ii) mediante la distribución integrada de la conectividad inalámbrica en la red de acceso. Esta integración de la distribución se realiza de forma eficiente a través de la transmisión óptica de las señales analógicas radio para su posterior detección y radiación hasta el usuario final, mediante el enlace vía radiofrecuencia correspondiente. Si además la transmisión óptica de estas señales inalámbricas se realiza en su formato final, en base a las frecuencias recogidas por la regulación pertinente, se denomina tecnología radio-sobre-fibra ( $\mathrm{RoF}$, Radio-over-Fibre). Este tipo de tecnología, la cual se describe en la sección 2.3, es una posibilidad a considerar dentro las redes de nueva generación (NGN, Next Generation Networks) [Kaz07].

En este capítulo se estudia la tecnología utilizada en las redes ópticas de acceso, así como en las redes basadas en sistemas RoF, para poder identificar sus principales características a la hora de implementar redes de acceso integradas. A su vez, se exponen los diferentes estándares de comunicaciones inalámbricas, en este caso WiMAX y UWB, con el fin de definir los requisitos asociados a su despliegue y distribución en la red de acceso óptica, como son su régimen binario, el alcance máximo y las modulaciones utilizadas. Finalmente se describen técnicas avanzadas de mitigación de la interferencia, consideradas en el futuro despliegue de sistemas de comunicaciones UWB, con el objetivo de evaluar su posible integración en las redes de acceso. 


\subsection{Redes de acceso cableadas}

Las redes de acceso cableadas establecen la comunicación desde la red troncal al usuario final. Esta red también es conocida como última milla (last mile). Se considera usuario final tanto a un usuario particular, como a una empresa o a instituciones públicas con redes de usuarios internos interconectados.

El concepto de usuario final ha cambiado en los últimos años, puesto que ha dejado de ser sólo una conexión física localizada en una ubicación estática determinada, para convertirse en una red de usuario que proporciona conectividad en movimiento. Así pues, el objetivo último de las redes de acceso es prestar servicios en áreas donde están presentes estos usuarios. Estas redes de usuario localizadas tras las redes de acceso pueden ser redes de área local, (LAN, Local Area Network), redes de comunicaciones personales (PAN, Personal Area Network), de ámbito humano (HAN, Human Area Network) o incluso redes corporales (BAN, Body Area Network), donde, a diferencia de los casos anteriores, el medio de transmisión es la superficie del cuerpo humano, o también una combinación de ellas.

Las redes de acceso cableadas actuales provienen en su mayoría de las infraestructuras heredadas de los operadores telefónicos tradicionales, puesto que la red de telefonía conmutada desplegó inicialmente el acceso al cliente en el bucle local de abonado. Esto se refleja en que la mayoría de redes de acceso están formadas por par trenzado de cable de cobre y sólo desde hace relativamente poco tiempo se ha empezado a utilizar la fibra óptica.

La red conmutada se empleó para transmitir voz modulada analógicamente, en el margen de frecuencias entre $300 \mathrm{~Hz}$ y $3400 \mathrm{~Hz}$, y posteriormente se digitalizó este tráfico, permitiendo regímenes binarios de $54 \mathrm{kbit} / \mathrm{s}$ con módems bajo la norma v.90. Este proceso de digitalización evolucionó hacia la red digital de servicios integrados (RDSI), permitiendo la transmisión simultánea de voz y datos con regímenes binarios de hasta $2048 \mathrm{kbit} / \mathrm{s}$ según la normativa [ETSI96]. El sistema RDSI posibilitó la digitalización completa de la red de acceso, puesto que el tramo de red metropolitana comprendida por las centrales locales de los operadores ya estaba digitalizado desde los años 80, bajo la norma de la jerarquía digital plesiócrona, (PDH, Plesiochronous Digital Hierarchy) y el protocolo de transferencia asíncrona (ATM, Asynchronous Transfer Mode). A principios de los años 90, evoluciona hacia la jerarquía digital síncrona (SDH, Synchronous Digital Hierarchy) en Europa y la red óptica síncrona (SONET, Synchronous Optical Network) en EEUU principalmente.

El paso siguiente en la evolución del bucle de abonado ha sido la introducción de las tecnologías de línea de abonado digital (DSL, Digital Subscriber Line) basadas en 
modulación digital multiportadora, especialmente adecuadas para la transmisión sobre par trenzado de cobre. En la actualidad existen diversas variantes DSL. Por ejemplo, el DSL asimétrico (ADSL, Asymmetrical Digital Subscriber Line), orientado al mercado residencial debido a la asimetría del régimen binario proporcionado, siendo mayor en el sentido "hacia el usuario" (sentido descendente o downstream), que en el sentido "hacia la central local" (sentido ascendente o upstream). ADSL permite regímenes binarios de 8.128 Mbit/s en el sentido descendente y $640 \mathrm{kbit} / \mathrm{s}$ en el sentido ascendente [ANSIT1413], [ITUG992.1]. Actualmente, la versión ADSL2+ permite regímenes binarios de hasta $24 \mathrm{Mbit} / \mathrm{s}$ hacia el usuario y de $2 \mathrm{Mbit} / \mathrm{s}$ desde el usuario [ITUG992.5]. Estas tecnologías, al usar el par trenzado como medio de transmisión, se ven afectadas por la limitación en alcance máximo, debida a la atenuación y a la presencia de interconexiones y segmentos de línea de par trenzado sin cargar, lo que se traduce en una respuesta en frecuencia desfavorable a la transmisión. Por ejemplo, en ADSL2+, la distancia entre el usuario y la centralita telefónica no supera los $3 \mathrm{~km}$ [ITUG992.5]. La tecnología DSL de muy elevado régimen binario (VDSL, Very High Bitrate Digital Subscriber Line) permite regímenes binarios de hasta $26 \mathrm{Mbit} / \mathrm{s}$ en su configuración simétrica, y regímenes binarios de $52 \mathrm{Mbit} / \mathrm{s}$ en sentido descendente y hasta $12 \mathrm{Mbit} / \mathrm{s}$ en sentido ascendente en su configuración asimétrica [Nav02].

El siguiente paso en la evolución en las redes de acceso cableadas es el uso de cable coaxial para la provisión de servicios de televisión por cable (CATV, Cable Television) principalmente. Estas redes presentan una estructura en árbol que requiere un gran número de amplificadores y divisores para cubrir áreas extensas con numerosos usuarios. A fin de mejorar la arquitectura de red, es posible utilizar las redes coaxiales junto a interconexiones de fibra óptica, dando lugar a las redes híbridas fibra y coaxial (HFC, Hybrid-Fibre Coaxial) [Way99]. En estas redes, el cable coaxial es el medio mediante el cual se llega al punto de entrada o conexión con el cliente. Las redes HFC pueden entenderse como un paso intermedio hacia el siguiente estadio de redes de acceso, las redes de acceso todo-óptico en las cuales la fibra óptica llega hasta el usuario final. 


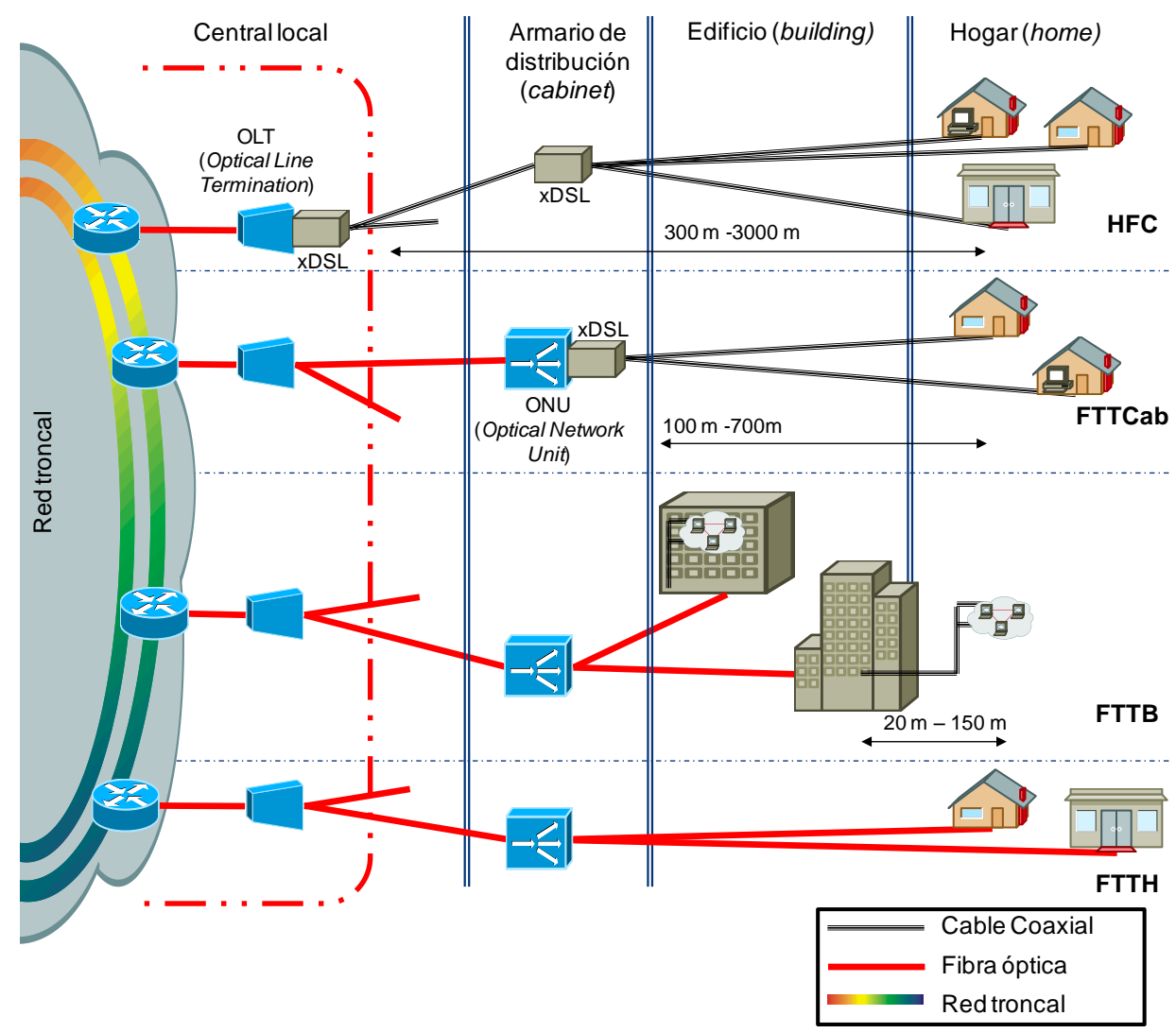

Figura 2.1. Esquemas de distribución FTTx y nomenclatura.

Las redes ópticas de acceso son denominadas de forma distinta dependiendo del punto de llegada del despliegue de fibra óptica realizado por el operador. En el caso de que la fibra óptica llegue hasta el edificio y luego se distribuya mediante cable coaxial, se denomina fibra hasta el edificio (FTTB, Fibre-to-the-Building). En cambio, si la fibra óptica llega hasta un armario de distribución, un área de clientes o una manzana de edificios, y luego continúa mediante cable coaxial hasta el usuario final, se denomina fibra hasta el armario de distribución (FTTCab, Fibre-to-the-Cabinet, FTTE, Fibre-to-the-Enclosure), fibra hasta la acera (FTTC, Fibre-to-the-Curb) y fibra hasta el nodo del barrio (FTTN, Fibre-to-the-Node/Neighbourhood), respectivamente. Finalmente, si la fibra óptica llega hasta el usuario final, se denomina fibra hasta el hogar (FTTH, Fibre-to-the-Home). El término general FTTx (Fibre-to-the- $x$ ) engloba a todos los términos anteriores. En la Figura 2.1 se pueden observar gráficamente estas variantes en el esquema de distribución FTTx. A su vez, en esta figura se observa la longitud de cable coaxial utilizado en cada esquema de distribución HFC o FTTx, y los dispositivos de distribución de tecnologías DSL, denominados xDSL. 
Las redes FTTx han tenido un fuerte impulso en los últimos años, como demuestran las tasas de penetración mostradas en la Figura 2.2. Este crecimiento también se refleja a nivel mundial con tasas de penetración FTTx del 5\% en EEUU, del $26 \%$ en Japón o del $44 \%$ en Corea del Sur.

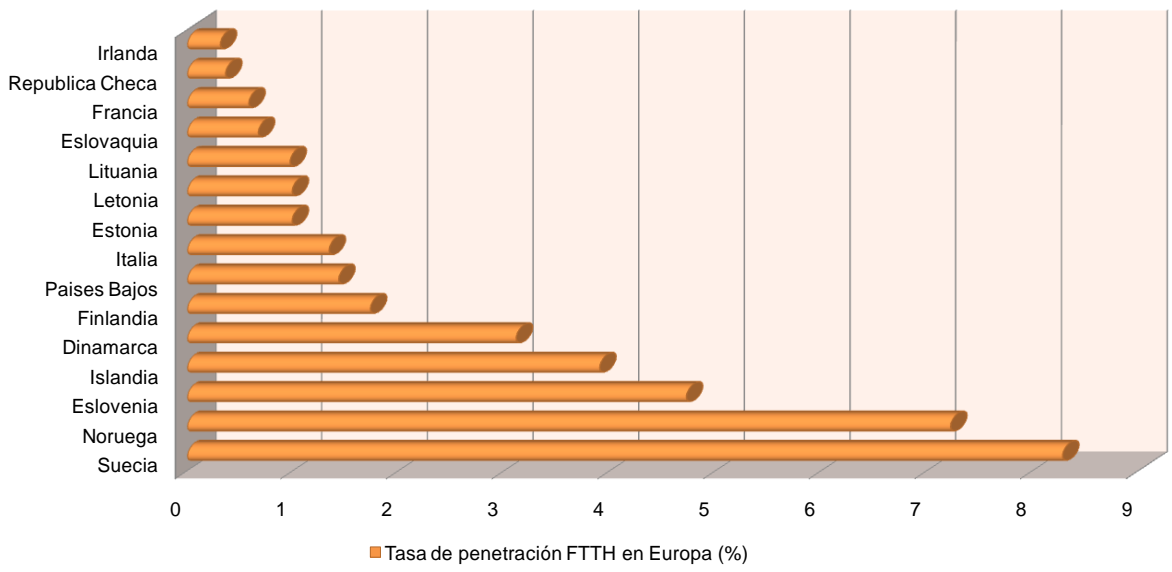

Figura 2.2.Tasa de penetración de la tecnología FTTH por número de hogares conectados en Europa, Octubre 2008 (Fuente: FTTH Council Europe).

Estas tasas de penetración indican que existe una ventaja en costes y es posible un modelo de negocio favorable utilizando FTTx. Por supuesto, este modelo de negocio considera los ingresos adicionales obtenidos de la distribución conjunta de servicios de voz, datos y vídeo denominado triple-play [Far02]. Este modelo puede extenderse mediante la agregación de servicios inalámbricos como WiMAX, dando lugar al denominado cuádruple-play. A su vez, la inclusión de otros servicios como los de tipo domótico o de seguridad en el hogar quedan englobados en el denominado quíntuple-play. La viabilidad del modelo de negocio se basa en distintos factores. En primer lugar, la reducción de costes que en los últimos años se ha producido en los equipos de FTTx por el factor de escala en su producción, especialmente en Asia. En segundo lugar, el coste de FTTx en nuevas áreas se ha equiparado al de las redes HFC, debido también a la facilidad de actualización y reutilización de este modelo de negocio, que permite obtener un valor añadido al planificar nuevos despliegues en zonas densamente pobladas. Por último, otro factor ha sido sin duda la madurez adquirida por esta tecnología con el desarrollo de recomendaciones relevantes por parte de la ITU-T (International Telecommunication Union - Telecommunication Standardization Sector), tales como ITU-T G.983 y ITU-T G.984 [ITUG983], [ITUG984]. Estas recomendaciones son consecuencia del trabajo realizado por los grupos internacionales de trabajo en redes ópticas como Full Service Access Networks [FSAN] y Ethernet in the First Mile [EFM]. Esta estandarización se ha trasladado a configuraciones 
específicas de FTTH denominadas redes ópticas pasivas (PON, Passive Optical Networks) [Cha06].

En la actualidad existen dos implementaciones mayoritarias en las redes ópticas de acceso, las punto-a-punto (PtP, Point-to-Point) y las punto-a-multipunto (PtMP, Point-to-Multipoint). Las soluciones PtP usan conexiones dedicadas de fibra óptica desde la central local, punto denominado "terminación de línea óptica" (OLT, Optical Line Termination), hasta el usuario final, pudiendo alcanzar el enlace hasta $80 \mathrm{~km}$ de distancia [Cha06]. Lógicamente, el coste de su despliegue es alto. En el caso de redes FTTB este coste se reduce gracias a que un grupo de usuarios comparte el enlace dedicado y los transductores del enlace. Este tipo de redes PtP se basan principalmente en Media Converters (MC) [Lin86], dispositivos que traducen electro-ópticamente la información para su transmisión óptica y soportan transmisiones desde Ethernet 10Base-T hasta Gigabit Ethernet (GbE) [EFM]. El uso de estos dispositivos permite una gestión remota por parte del operador, al incluirla dentro de los nodos Ethernet, aunque el mantenimiento de los nodos activos supone un coste mucho mayor [Cha06]. Por otro lado, las soluciones PtMP se basan principalmente en tecnologías PON para ofrecer servicios de elevado régimen binario en redes de acceso [Ste87]. Las redes PON se caracterizan por no incluir elementos activos (amplificadores, regeneradores) y presentar una topología de red en árbol, que minimiza el número de divisores ópticos, reduciendo las pérdidas de potencia y aumentando el alcance máximo de la red. La topología en árbol 1-a-N usuarios implica que el coste del OLT de red es compartido por todos los usuarios y también reduce el número de transceptores electro-ópticos (E/O) requeridos. La inclusión de técnicas de multiplexación por división en tiempo (TDM, Time Division Multiplexing) y en longitudes de onda (WDM, Wavelenght Division Multiplexing) en las redes PON facilita el acceso múltiple a todos los usuarios [Par04].

Las redes PON actuales están basadas en las iniciativas del grupo FSAN y EFM, que se han convertido en los estándares utilizados actualmente en FTTx. Las redes FTTx basadas en la iniciativa FSAN utilizan el protocolo ATM; las redes PON basadas en ATM se denominan ATM-PON (APON) [ITUG983]. Las redes APON se caracterizan por permitir divisores de hasta $1 \times 32$, con alcances de hasta $20 \mathrm{~km}$, proporcionando regímenes binarios por usuario de $155 \mathrm{Mbit} / \mathrm{s}$ y $622 \mathrm{Mbit} / \mathrm{s}$ en el enlace descendente y de $155 \mathrm{Mbit} / \mathrm{s}$ en el enlace ascendente. Dentro de este estándar se sitúan las denominadas redes PON de banda ancha (BPON, Broadband-Passive Optical Network), que reservan una o varias longitudes de onda para distribuir vídeo en el enlace descendente mediante una sola portadora de radiofrecuencia, que al estar localizada en la misma banda que los operadores tradicionales de CATV, en $1550 \mathrm{~nm}$, simplifica su distribución. Para ello, el espectro óptico en el enlace descendente se divide en 4 bandas permitiendo transmisión de vídeo, de servicios digitales basados en 
DWDM [ITUG959.1], tráfico de datos y reserva de longitudes de onda para futuros usos. Otra recomendación posterior de FSAN se tradujo en los estándares ITU-T G.984 [ITUG984] para redes PON Gigabit (GPON, Gigabit Passive Optical Network). Las redes GPON permiten divisores de hasta 1x128 y regímenes binarios simétricos de hasta $2.488 \mathrm{Gbit} / \mathrm{s}$. A su vez, estas redes aumentan la seguridad en el tráfico de datos con una encriptación basada en el algoritmo AES, y proporcionan una integración a nivel de trama que permite evitar protocolos de encapsulación.

Por otro lado, la iniciativa EFM derivó en el grupo de trabajo del IEEE para Ethernet en redes ópticas, cuyo estándar es IEEE 802.3ah [EFM]. Puesto que Ethernet es el formato de tráfico de datos más común en las redes locales y por lo tanto es el tráfico que generan las redes que forman Internet en su gran mayoría, es interesante la integración de ambos dominios. En 2004, se estandarizan las redes PON basadas en Ethernet (EPON, Ethernet-Passive Optical Network) mediante el estándar IEEE 802.3ah. En este estándar se recoge la nomenclatura ya propuesta por FSAN en G.983.x para evitar confusiones innecesarias, así como sus aportaciones en cuanto a longitudes de onda, tipos de fibra y longitudes de fibra. El IEEE 802.3ah permite alcances superiores a los $10 \mathrm{~km}$ en fibra monomodo para velocidades de $1.25 \mathrm{Gbit} / \mathrm{s}$, utiliza el mismo rango de longitudes de onda que el G.983.3 y sobre todo usa el formato de trama Ethernet en los paquetes de datos de esas redes. En este estándar se definen las redes PON basadas en Ethernet y las actuales basadas en Gigabit Ethernet (GEPON, Gigabit Ethernet-Passive Optical Network).

La inclusión de la tecnología WDM en las redes PON implica la utilización de diversas longitudes de onda óptica en una misma fibra para transmitir la información desde el OLT hasta el usuario final, en el punto de unidad óptica de red (ONT, Optical Network Unit). En recepción, cada longitud de onda se separa mediante dispositivos WDM y no mediante divisores de potencia óptica. Básicamente, se trata de un nuevo esquema en PtP, pero también puede ser llevado a situaciones PtMP. Esto se consigue de la siguiente forma: en lugar de tener un usuario final en ONT, se incorporan las redes PON basadas en TDM, con lo que se consigue dar servicio a múltiples usuarios y reducir los costes compartidos, al tener dispositivos láser dedicados para cada grupo de clientes por cada una de las redes superpuestas. Al mismo tiempo, este tipo de redes requiere de una sola fibra óptica para el tráfico de subida y de bajada, y reduce la complejidad al transmitir varios sistemas TDM sobre PON sobre una sola fibra. Este tipo de acceso se puede denominar acceso mediante WDM (WDMA) [Wag89]. La investigación en este tipo de redes ha permitido superar la barrera del alto coste implícito a la utilización de fuentes láser para cada longitud de onda, tanto en el OLT como en el ONT. Sin embargo, esto conlleva problemas de mantenimiento debido a la estabilidad con respecto a la temperatura o el consumo y el mantenimiento en el OLT y en el ONT. En general, el desarrollo de las redes PtMP WDM-PON tiene como 
principales ventajas: (i) mayor régimen binario disponible en cada ONT mediante una sola longitud de onda, (ii) aumento de la seguridad al utilizar enlaces WDM, (iii) balance de potencia más favorable, ya que sólo introduce 9-12 dB de perdidas por cada 16-32 usuarios servidos, y (iv) posibilidad de dar servicio a más usuarios por cada longitud de onda, cuestión intrínseca al superponer una red PON, teniendo redes de 1 x $N$ x $M$ usuarios [Shu08], siendo $N$ el número de redes PON y $M$ el número de usuarios de cada red PON.

El desarrollo de redes WDM-PON posibilita el desarrollo de redes más flexibles desde el punto de vista del ONT, permitiendo así dispositivos independientes de la longitud de onda (colorless) [Gro08]. En este tipo de redes se han propuesto fuentes láser de espectro muy ancho que son filtradas seleccionando la parte del espectro necesaria para cada ONT, este tipo de fuentes son del tipo diodos laser luminiscentes (LED, Light Emitting Diode). La ventaja asociada al uso de estas fuentes es su sencilla implementación y bajo coste. Sin embargo, la incoherencia óptica de estas fuentes no permite su uso conjunto si no han sido filtradas previamente, lo cual limita la flexibilidad de las redes y aumenta la posibilidad de colisiones. A su vez, las fuentes LED poseen baja potencia óptica y una limitada velocidad de modulación [Fri98]. Otras soluciones colorless se basan en el uso de fuentes Fabry-Perot (FP), que inyectan la señal óptica al ONU y luego la reciben de nuevo para transmitirla al OLT. En cada uno de estos casos, se sintoniza una longitud de onda en el FP para que actúe como una fuente de una sola longitud de onda, reduciendo el coste del sistema. Sin embargo, los FP de inyección-cerrada presentan limitaciones en cuanto al rango de longitudes sintonizables y la potencia requerida para ello [Lee05]. Cabe destacar el uso de amplificadores ópticos de semiconductor reflexivos (RSOA, Reflective Semiconductor Optical Amplifier) para permitir una mayor transparencia en la WDM-PON. En estos casos no existen fuentes láser en el ONT, puesto que la fuente láser se encuentra centralizada en el OLT, y una vez que las longitudes de onda llegan al ONT se reutilizan mediante moduladores externos o RSOA, con lo cual se puede enviar de nuevo la información en el enlace de subida con la misma señal óptica recibida. El uso de RSOA conlleva una mayor ganancia que con el uso de moduladores externos convencionales. Esta solución reduce el número de fuentes láser y de longitudes de onda, por lo que la gestión del sistema es mucho más fluida debido a tener un entorno más controlado y centralizado en el OLT. Sin embargo, estos sistemas basados en RSOA presentan sensibilidad a la polarización y un aumento en el coste de la gestión de este tipo de redes transparentes WDM-PON [Pra05a], [Are06].

En resumen, la demanda de servicios de elevado régimen binario ha supuesto el punto de inflexión definitivo para el desarrollo de las redes de acceso cableadas. Esto ha permitido el desarrollo de tecnologías para las infraestructuras de par trenzado de cobre ya instaladas, como por ejemplo las tecnologías VDSL, que permiten transmisiones de 
hasta $20 \mathrm{Mbit} / \mathrm{s}$ en áreas de hasta un kilómetro desde la centralita telefónica al usuario final. Paralelamente, también se han desarrollado las tecnologías HFC, que permiten regímenes binarios cercanos a los 10/20 Mbit/s en áreas de hasta medio kilómetro desde la terminación de fibra óptica. En la actualidad, las redes FTTx presentan un gran crecimiento, como muestra la Figura 2.2 con la representación de las tasas de penetración de las redes FTTH. Por ello, las redes ópticas se presentan como la tecnología adecuada para dar respuesta a la demanda futura de servicios. En la Tabla 2.1 se resumen las actuales tecnologías de redes de acceso por cable y fibra óptica, así como sus características de alcance máximo y régimen binario por usuario.

TABLA 2.1. RÉGIMEN BINARIO POR USUARIO Y ALCANCE MÁXIMO EN DISTINTAS TECNOLOGÍAS DE ACCESO CABLE

\begin{tabular}{lcc}
\hline \hline Servicio & Régimen binario/Usuario & Alcance máximo \\
\hline ADSL & $2 \mathrm{Mbit} / \mathrm{s}(\mathrm{estándar})$ & $5.5 \mathrm{~km}$ \\
\hline ADSL2+ & $16 \mathrm{Mbit} / \mathrm{s}$ & $1.5 \mathrm{~km}$ \\
\hline VDSL & $20 \mathrm{Mbit} / \mathrm{s}(\mathrm{estándar})$ & $1 \mathrm{~km}$ \\
\hline VDSL2 & $50 \mathrm{Mbit} / \mathrm{s}$ & $1 \mathrm{~km}$ \\
\hline Coaxial & $2 \mathrm{Mbit} / \mathrm{s}$ & $0.5 \mathrm{~km}$ \\
\hline BPON & $20 \mathrm{Mbit} / \mathrm{s} *$ & $20 \mathrm{~km}$ \\
\hline EPON & $60 \mathrm{Mbit} / \mathrm{s}$ & $20 \mathrm{~km}$ \\
\hline GPON & $40 \mathrm{Mbit} / \mathrm{s} *$ & $20 \mathrm{~km}$ \\
\hline \hline $\begin{array}{r}\text { régimen binario calculado en base a número de usuarios } \\
\text { considerados en los estándares pertinentes }\end{array}$
\end{tabular}

\subsection{Redes de usuario vía radiofrecuencia}

Las comunicaciones inalámbricas y móviles se encuentran en continuo crecimiento. Cabe destacar que sólo las comunicaciones móviles llegan a más del $70 \%$ de la población mundial, basándose tanto en estándares 2G (GSM, EGPRS) como en $3 \mathrm{G}$ (UMTS, HSPA). A su vez, existen futuros estándares ya en plena evaluación (LTE). La demanda de servicios de acceso a redes de datos y comunicaciones de tipo móvil auspicia el desarrollo de nuevas tecnologías radio. Debido a la heterogeneidad de los escenarios de aplicación, cualquier nueva tecnología radio debe proveer el acceso al usuario de forma independiente al escenario de aplicación.

Las redes de usuarios vía radiofrecuencia actuales deben responder a la demanda de acceso a servicios de elevado régimen binario mediante conexiones inalámbricas. Así pues, según el escenario de aplicación, nos encontramos desde las redes inalámbricas personales (WPAN, Wireless Personal Area Networks), para áreas menores de 10 metros, hasta redes inalámbricas extensas (WWAN, Wireless Wide Area Networks). En cada uno de estos escenarios, son varias las tecnologías que facilitan el acceso a los 
servicios, desde tecnologías de comunicaciones móviles que cubren territorios extensos geográficamente, como son las tecnologías 3G HSPA [3GPP], hasta aquellas que permiten el acceso de forma inalámbrica en escenarios de corta distancia WLAN, como son aquellas basadas en IEEE 802.11 (Wi-Fi) [IEEE80211]. Esta distribución según área de cobertura de las tecnologías de acceso radio, junto a la creciente demanda de servicios de elevado régimen binario, supone la extensión de las redes de acceso tradicionales a través de redes de usuario vía radiofrecuencia. Este tipo de redes permite un acceso móvil y ubicuo. Por otra parte, la creciente implantación de dispositivos que integran el acceso mediante tecnologías vía cable y vía radio en un mismo equipo, es un ejemplo de la tendencia hacia la integración universal del acceso de forma transparente al usuario. En este contexto, las redes vía radiofrecuencia son clave para implementar el acceso universal y asegurar el acceso en cualquier lugar en las mejores condiciones a cualquier usuario.

En los escenarios de redes WPAN nos encontramos con tecnologías radio de corto alcance, menores a 10 metros, lo cual, unido a su baja complejidad, bajo consumo de potencia y bajo coste, ha facilitado su rápida proliferación. En WPAN se pueden conformar grupos de usuarios cercanos (hot-spots) que comparten bandas de frecuencias sin licencia, como por ejemplo $2.4 \mathrm{GHz}$. Entre estas tecnologías WPAN, cabe destacar a la familia IEEE 802.15.1 o Bluetooth [IEEE80215.1], cuyo objetivo principal es el reemplazo de cables para el intercambio de datos entre dispositivos y el control de periféricos con regímenes binarios de hasta $3 \mathrm{Mbit} / \mathrm{s}$. Por otra parte, la tecnología ZigBee se implementa en WPAN para comunicaciones de radios digitales de bajo consumo. Esta tecnología implementa un bajo régimen binario en sus transmisiones y maximiza la vida útil de las baterías. ZigBee se despliega en entornos de comunicación industrial, facilitando servicios de comunicación entre dispositivos de tipo domótico, de procesos automatizados industriales y la adquisición de datos médicos como la monitorización de pacientes. Todos estos ejemplos de comunicaciones basadas en ZigBee poseen regímenes binarios de hasta $250 \mathrm{kbit} / \mathrm{s}$. ZigBee se considera dentro del grupo de tecnologías IEEE 802.15.4 [IEEE80215.4]. En los escenarios WPAN también existen tecnologías radio de elevado régimen binario, como la tecnología radio de banda ultra-ancha (UWB). UWB es una tecnología radio de corto alcance, hasta 10 metros, y elevados regímenes binarios, hasta $1 \mathrm{Gbit} / \mathrm{s}$ [ECMA-368]. UWB opera en las bandas de frecuencias de 3.1-10.6 GHz, $22-24 \mathrm{GHz}$ y $57-64 \mathrm{GHz}$, y transmite con anchos de banda superiores a los $50 \mathrm{MHz}$. UWB es una tecnología que se considera dentro del grupo de trabajo IEEE 802.15.3 [IEEE80215.3]. Estas características, unidas a su bajo consumo y su baja interferencia con otras tecnologías inalámbricas, sitúan a la tecnología de comunicaciones UWB como una candidata firme para la transmisión de datos a elevados regímenes binarios en escenarios de corto alcance para aplicaciones como la transmisión inalámbrica de audio y vídeo de alta-definición y el nuevo estándar inalámbrico de USB (WUSB). 
En los escenarios de tipo WLAN, con alcances menores a 100 metros, como oficinas, hogares, hot-spots en zonas de gran densidad de usuarios como aeropuertos, la tecnología predominante es IEEE 802.11, ya sea como parte de una estructura de distribución y acceso o de forma independiente ad-hoc. Actualmente, estas tecnologías operan en las bandas de frecuencia de $2.4 \mathrm{GHz}$ y $5 \mathrm{GHz}$, las cuales se consideran ISM (ISM, Industrial, Scientifical and Medical) y no requieren licencia de explotación. Esta tecnología permite extender Ethernet sobre el acceso inalámbrico, desde regímenes binarios de $54 \mathrm{Mbit} / \mathrm{s}$ mediante IEEE 802.11a, y hasta regímenes binarios de $248 \mathrm{Mbit} / \mathrm{s}$ en base a técnicas MIMO y IEEE $802.11 \mathrm{n}$, con alcances de hasta $150 \mathrm{~m}$. Las redes WLAN basadas en IEEE 802.11 implementan la capa física y de enlace de datos del modelo ISO, lo cual permite implementar el acceso inalámbrico de servicios de elevado régimen binario para redes de datos cableadas.

Las redes de radiofrecuencia que se despliegan en escenarios de corta distancia proporcionan acceso a la demanda de servicios de elevado régimen binario de forma inalámbrica. En estos escenarios de aplicación surgen las tecnologías para acceso inalámbrico de banda ancha (BWA, Broadband Wireless Access) bajo el estándar IEEE 802.16, conocido comúnmente como WiMAX. Esta serie de estándares definen el acceso radio de banda ancha fijo (FWA, Fixed Broadband Wireless Access) para áreas extensas de usuarios con regímenes binarios de hasta $134 \mathrm{Mbit} / \mathrm{s}$ y alcances de hasta $50 \mathrm{~km}$. Por otra parte, estas tecnologías se implementan en las banda de frecuencias de 10.6 GHz-66 GHz para comunicaciones radio mediante enlaces de visión directa (LOS, Line of Sight) de varias decenas de kilómetros de alcance en base al estándar IEEE 802.16d [IEEE80216d]. Este tipo de comunicaciones implementan enlaces punto-a-punto en el bucle de abonado para zonas de difícil acceso o zonas rurales con baja densidad de población. En situaciones sin LOS, las comunicaciones WiMAX se implementan en la banda de frecuencias de $2 \mathrm{GHz}-10 \mathrm{GHz}$ conforme al estándar IEEE 802.16e o Mobile WiMAX [IEEE80216e]. En el estándar IEEE 802.16d se contemplan comunicaciones radio de hasta $1 \mathrm{Gbit} / \mathrm{s}$ en enlaces LOS de varios $\mathrm{km}$, mientras que Mobile WiMAX se contempla comunicaciones en escenarios de movilidad con hasta $100 \mathrm{Mbit} / \mathrm{s}$ para varios cientos de metros de cobertura. El futuro desarrollo de WiMAX pretende incorporar parámetros mejorados de calidad de servicio (QoS, Quality-of-Service) para la realización de transferencia de usuarios entre redes. La implementación de estas características indican que WiMAX pretende ampliar su cobertura a redes WWAN, en las cuales se sitúan las redes de comunicaciones móviles $3 \mathrm{G}$.

Finalmente, cabe destacar que en las redes WWAN se está produciendo una evolución hacia una futura cuarta generación de telefonía móvil, la cual deberá estar definida en 2011 bajo las recomendaciones IMT-Advance 4G (IMT, International Mobile Telecommunications). En este punto, es necesario destacar que existen 
recomendaciones auspiciadas por el 3GPP, derivadas del actual desarrollo de LTE-Advance, y aquellas que se recogen en el nuevo IEEE 802.16m o WiMAX II, auspiciadas por las autoridades norteamericanas. Ambas recomendaciones son propuestas de estándares de comunicaciones móviles 4G, cuyo objetivo son las comunicaciones móviles de regímenes binarios mayores a $1 \mathrm{Gbit} / \mathrm{s}$ en WWAN y que marcarán la evolución de las comunicaciones móviles en los próximos años. En la Figura 2.3 se representa todo el conjunto de tecnologías de acceso inalámbrico actuales según el criterio de área de aplicación o cobertura.

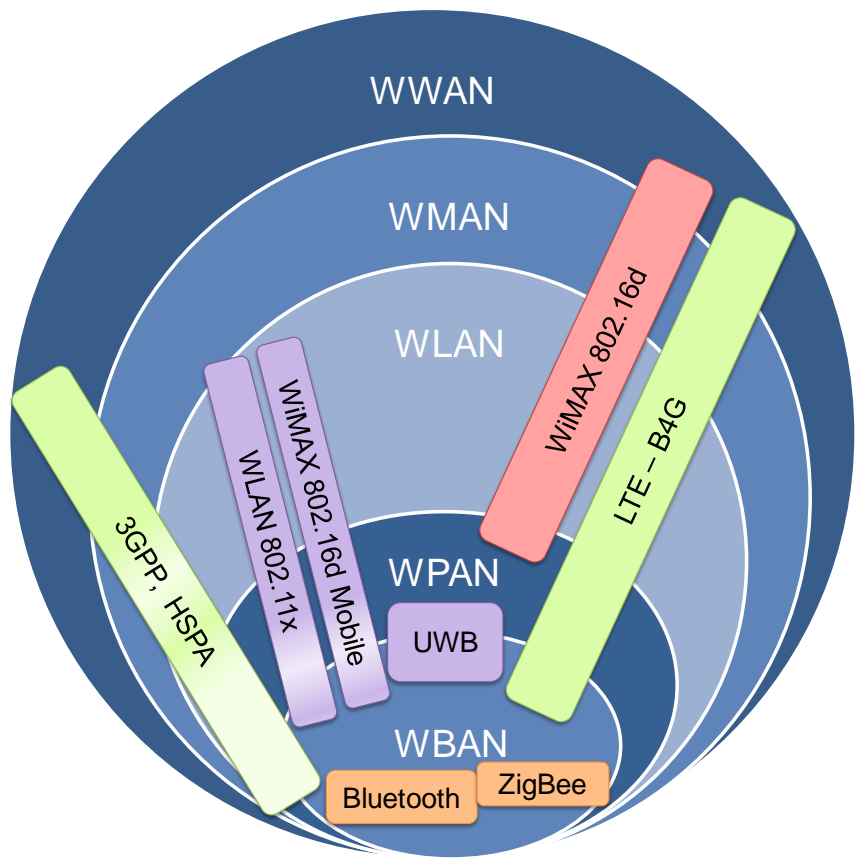

Figura 2.3.Tecnologías de comunicaciones vía radio según área de aplicación, de menor a mayor radio de cobertura.

Respecto al alcance, las redes vía radiofrecuencia actuales contemplan múltiples escenarios, desde coberturas celulares de área extensa mediante comunicaciones móviles $3 \mathrm{G}$, hasta entornos personales para la comunicación directa entre dispositivos como las transmisiones de datos Bluetooth. El principal reto al cual se enfrentan las redes vía radiofrecuencia es la capacidad de dar servicio a los usuarios en los diferentes escenarios, sujetos a distintas regulaciones. Por ejemplo, en escenarios de tipo WLAN, el reto se sitúa en aumentar el régimen binario de las comunicaciones desplegadas en las bandas de frecuencia ISM, que se encuentran sobreexplotadas. Este aumento se consigue mediante el uso de nuevas frecuencias, como son las bandas de frecuencias de 57-64 GHz o de $23 \mathrm{GHz}$, mediante modificaciones en las tecnologías radio actuales, 
buscando modulaciones más eficientes que reaprovechen el espectro, o mediante la reducción del alcance mediante redes pico- o femto-celulares en escenarios WLAN.

\subsection{Sistemas radio-sobre-fibra}

La tecnología RoF permite conjugar las redes ópticas de acceso y su gran ancho de banda con las redes vía radiofrecuencia y su gran flexibilidad. La transparencia y la flexibilidad son características de los sistemas RoF, por lo que se las considera una excelente herramienta para afrontar el desarrollo y evolución de las redes de acceso y facilitar el acceso inalámbrico de elevado régimen binario de forma flexible, muy versátil y con bajo coste [Oga92], [Wak02a]. Los sistemas RoF centralizan la generación y control de las señales de radiofrecuencia a transmitir mediante fibra óptica hacia antenas remotas o puntos de acceso inalámbricos, para su posterior acceso según la tecnología radio empleada.

La Figura 2.4 presenta un ejemplo de distribución de señales radio mediante un sistema RoF. En este escenario de aplicación la arquitectura de distribución RoF consta de cuatro bloques principales, una estación central (CS, Central Station), una serie de estaciones base (BS, Base Station), enlaces de fibra óptica y un terminal de cliente (CPE, Customer Premises Equipment) que permite el acceso del usuario, ya sea para su retransmisión, redistribución interior u otros aspectos del acceso al servicio. En este esquema de aplicación se puede diferenciar entre el enlace descendente, el establecido desde la CS hacia el usuario, y el enlace ascendente, el realizado desde el usuario hacia la CS. En el enlace descendente, se realiza una conversión en la CS de la señal transmitida de RF, desde frecuencias de RF a frecuencias ópticas del orden de $\mathrm{nm}$. Posteriormente, la señal óptica se propaga por la fibra hasta llegar a la BS. En la BS, la señal óptica se convierte de nuevo a señal de RF, tras lo cual es distribuida a través de cable coaxial hacia la antena emisora para su radiación hacia el CPE. En cambio, en el enlace descendente, la señal radio transmitida por el CPE se recibe en la antena y se transmite por cable coaxial hasta la BS, donde se realiza la conversión electro-óptica de la señal. Esta señal se transporta por la fibra óptica hasta la CS para su conversión a señal de radiofrecuencia y, tras un procesado electrónico, es retransmitida ópticamente hacia la red metropolitana correspondiente. De este modo, la complejidad se sitúa en la CS, lo cual permite la centralización y simplificación de la red. Este es el ejemplo más común, el cual puede variar trasladando parte de la inteligencia de red, como la conversión electro-óptica, a las antenas transceptoras, evitando así el tramo de cable coaxial y consiguiendo un sistema de distribución todo óptico antes de su distribución radio, aunque esto conlleve mayor complejidad en las antenas transceptoras y una simplificación de la BS. 


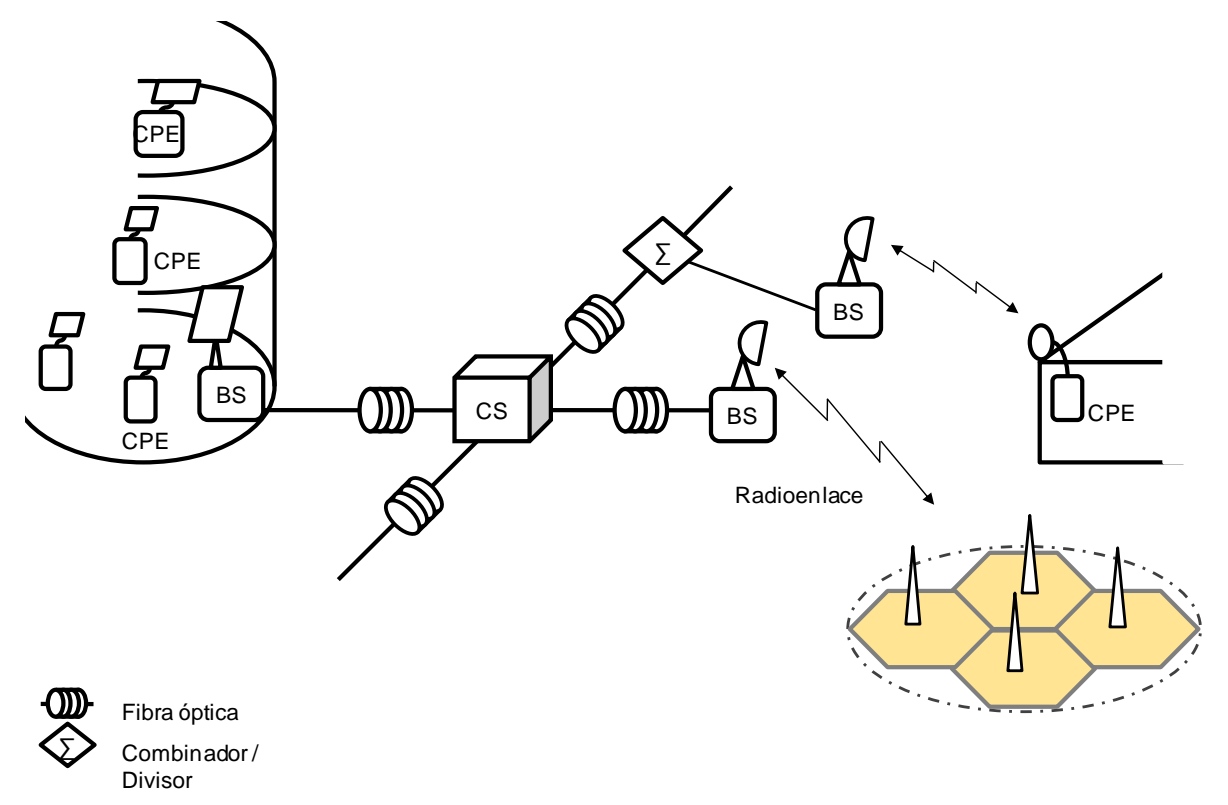

Figura 2.4. Arquitectura y escenario de aplicación de un sistema RoF.

Otro ejemplo de las ventajas de los sistemas RoF lo tenemos en los entornos micro/pico-celulares, como son los sistemas de antenas distribuidas (DAS, Distributed Antenna Systems) [Sal87b]. En estos sistemas se utilizan numerosos puntos de acceso, como por ejemplo antenas, conectados a una infraestructura de fibra óptica, que actúan como repetidores de la BS. Estos sistemas se plantearon inicialmente para extender y mejorar la cobertura en entornos interiores o de difícil acceso como túneles. Actualmente, los sistemas DAS responden a la demanda de acceso a diferentes servicios sobre el mismo sistema de distribución. Esta distribución requiere de una amplificación y gestión adecuada de los recursos radio según las necesidades del escenario [Cri09]. Un ejemplo de este tipo de sistemas lo tenemos en aeropuertos o en terminales de metro, donde se distribuyen servicios WLAN y se aumenta la cobertura $3 \mathrm{G}$ al mismo tiempo con la misma red de distribución de fibra óptica. Esto permite un uso eficiente de divisores y amplificadores ópticos.

Así pues, los sistemas RoF presentan una tecnología madura y de gran fiabilidad, que se emplea, por ejemplo, en aplicaciones científicas y militares, como es el procesado óptico de señales RF, en enlaces con antenas remotas, como por ejemplo para comunicaciones con satélites, o en la generación de frecuencias de milimétricas [See06]. Cabe destacar la utilización de la tecnología RoF para el transporte y gestión de señales entre estaciones base en redes de comunicaciones móviles 3G. 
En la siguiente sección se presentan los sistemas RoF atendiendo a los métodos usados para el transporte de la información radio.

\subsubsection{Multiplexación por división en subportadoras}

En este tipo de sistemas RoF, el transporte de la información se realiza mediante un múltiplex en frecuencia óptica de las señales a radiar posteriormente. Tradicionalmente, este tipo de sistemas de transmisión RoF se denominan de multiplexación por subportadoras (SCM, Sub-Carrier Multiplexing) [Ols98]. En los sistemas RoF con SCM, la señal RF se modula en intensidad sobre una portadora óptica, obteniendo en frecuencias ópticas una doble banda lateral alrededor de la portadora óptica donde se sitúan modulados los diferentes canales del múltiplex de RF, de ahí su denominación de subportadoras. La señal modulada ópticamente en intensidad por dispositivos electro-ópticos es transmitida por fibra óptica hasta llegar al terminal final, donde se detecta directamente mediante un foto-detector para su posterior radiación y distribución hacia el usuario final.
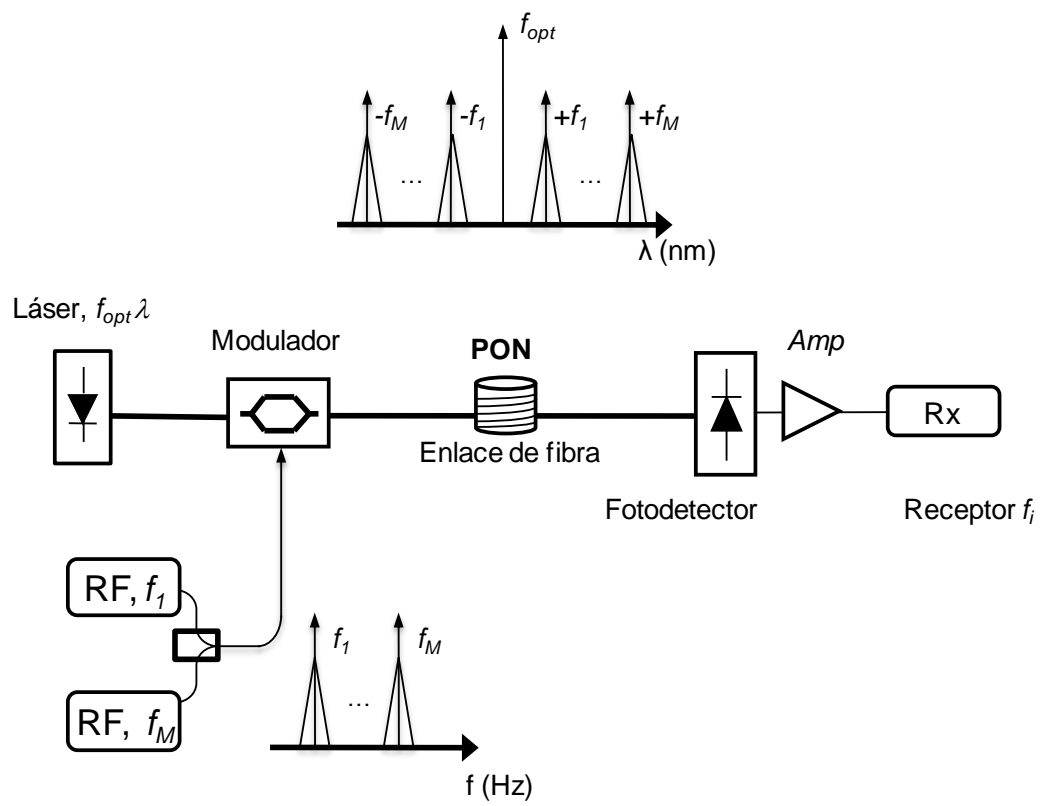

Figura 2.5. Concepto de sistema RoF con transmisión basada en SCM.

En la Figura 2.5 se presenta un esquema típico de sistema RoF basado en SCM. Un número de canales RF con frecuencias portadoras $f_{i}$ forma un múltiplex en frecuencia, el cual se modula en intensidad mediante un modulador externo Mach-Zehnder. En la señal óptica se distingue entonces una portadora óptica, generada por un láser, y bandas 
laterales donde se encuentra modulado ópticamente el múltiplex de RF. Esta señal óptica se transmite entonces a través de un enlace de fibra óptica, que habitualmente se corresponde con un sistema óptico de tipo PON. Finalmente, la señal óptica se detecta mediante un foto-detector adecuado al ancho de banda óptica de la señal modulada para realizar la conversión opto-eléctrica. Posteriormente, la señal eléctrica se amplifica y la $f_{i}$ del múltiplex de RF transmitido se demodula de acuerdo al canal deseado.

En el esquema de la Figura 2.5 se incluyen los principales elementos para el transporte de señales RF en sistemas RoF SCM: los elementos de conversión electro-óptica y opto-eléctrica, los láseres y los enlaces de fibra óptica. Estos elementos, introducen determinadas limitaciones sobre los sistemas RoF con SCM. La primera limitación la impone la atenuación de la fibra óptica, que se soslaya mediante el uso de amplificadores ópticos de banda ancha, como los EDFA (EDFA, Erbium Doped Fibre Amplifier) actuales. Otra limitación en los sistemas RoF viene impuesta por la fibra óptica, debido a su dispersión cromática asociada y a los efectos de no-linealidad o efecto Kerr [Agr02]. En sistemas digitales, estos efectos generan el ensanchamiento temporal de los símbolos y por lo tanto, introducen interferencia entre símbolos y aumentan la tasa de error. En sistemas analógicos con SCM, el efecto de la transmisión en fibra óptica se traduce tanto en el llamado efecto de supresión de portadora como en la distorsión por intermodulación. El efecto de supresión de portadora implica que para ciertas combinaciones de distancia de propagación en fibra óptica y frecuencia de modulación, no existe transmisión de la señal modulada. Por ello, este efecto limita el producto distancia por ancho de banda que caracteriza una fibra óptica. Cabe destacar que la no-linealidad de la fibra genera productos de intermodulación no deseados que degradan la señal propagada en la fibra, lo cual constituye una fuente de ruido para la transmisión óptica. En un sistema RoF basado en SCM, se ha de contabilizar la existencia de diversas fuentes de ruido que degradan la calidad de la señal transportada, como son los ruidos shot, térmico y el efecto de distorsión por intermodulación.

Para minimizar el efecto de la supresión de portadora, se han desarrollado técnicas como la introducción de una modulación de fase residual en los moduladores externos, comúnmente conocida como chirp, que permite incrementar el ancho de banda de modulación de la fibra [Pol99a]. También se ha propuesto el uso de redes difractivas de Bragg, y de fibras compensadoras de la dispersión, que implementan una dispersión inversa a la de la fibra óptica en transmisión [Nag01]. Por último, se ha estudiado la transmisión en bandas de frecuencias intermedia, que evita la degradación debida a la dispersión cromática de la fibra [Mar99], [Pol04]. 


\subsubsection{Multiplexación por división en longitudes de onda}

Este tipo de sistemas RoF transportan la señal RF en el dominio óptico mediante multiplexación por división en longitudes de onda (WDM, Wavelength Division Multiplexing). Esta técnica WDM incrementa la capacidad para el transporte óptico de señales al enviar diferentes múltiplex de señales RF en diferentes portadoras ópticas por la misma fibra. El interés en los sistemas WDM se debe al desarrollo de los amplificadores ópticos EDFA a mediados de los 90, que permiten amplificar un rango notable de longitudes de onda a la vez.

Aunque inicialmente se soportaban solo dos longitudes de onda a 1300 y $1550 \mathrm{~nm}$, actualmente se han llegado a amplificar simultáneamente hasta 160 longitudes de onda normalizadas por la ITU-T [ITUG694.1]. Esto ha permitido elevar la capacidad de transporte óptico a 40, 160 y $640 \mathrm{Gbit} / \mathrm{s}$, e incluso hasta 1 Tbit/s [Tsu01]. También cabe destacar la importancia de los formatos de modulación y codificación óptica de la señal en el aumento de la eficiencia de los sistemas WDM, sobre todo a partir del año 2000. Entre estos formatos tenemos detección coherente, multiplexación por división por polarización (PDM, Polarization Division Multiplexing), multiplexación mediante división en frecuencias ortogonales (OFDM, Orthogonal Frequency Division Multiplexing) y conformación de pulsos. Estas técnicas han permitido obtener eficiencias espectrales de transmisión de $9.3 \mathrm{bit} / \mathrm{s} / \mathrm{Hz}$ para transmisiones de $14 \mathrm{Gbit} / \mathrm{s}$ [Win05], de $5.6 \mathrm{bit} / \mathrm{s} / \mathrm{Hz}$ en $50 \mathrm{Gbit} / \mathrm{s}$ [Nak08] o de $4.2 \mathrm{bit} / \mathrm{s} / \mathrm{Hz}$ para $100 \mathrm{Gbit} / \mathrm{s}$ [San08]. Los estudios actuales sitúan la capacidad óptica de transmisión de un sistema WDM en una fibra monomodo en $11 \mathrm{bit} / \mathrm{s} / \mathrm{Hz}$ sobre una distancia de $2000 \mathrm{~km}$ [Ess09]. Este progreso en la eficiencia espectral de transmisión de los sistemas WDM, supone el consumo de múltiples recursos, lo cual impone una importante limitación en la eficiencia real de los sistemas WDM. Por ejemplo, el experimento que consigue una capacidad agregada de $25.6 \mathrm{Tbit} / \mathrm{s}$ con eficiencia espectral de $3.2 \mathrm{bit} / \mathrm{s} / \mathrm{Hz}$ [Gna07], consume 320 canales multiplexados en el tiempo electrónicamente (ETDM, Electronically Time-Division Multiplexing), cada uno con 2 bandas amplificadas ópticamente, 80 longitudes de onda por banda, 2 polarizaciones por longitud de onda y sobre cada polarización modulada a $80 \mathrm{Gbit} / \mathrm{s}$. Para poder aumentar la capacidad de los sistemas WDM actuales, se está investigando también la utilización de nuevos diseños de fibra no convencionales y de técnicas complementarias de multiplexación.

Las principales limitaciones de estos sistemas, al igual que en los sistemas SCM, son el efecto de las no-linealidad de la fibra óptica, el efecto de supresión de portadora y la no-linealidad de los amplificadores EDFA ópticos. Como se ha comentado anteriormente, se han propuesto múltiples esquemas y técnicas para evitar los efectos de supresión de portadora o atenuación por dispersión cromática, que en sistemas WDM, debido al uso de múltiples portadoras ópticas, es especialmente problemático. En estos 
sistemas, cabe destacar la utilización de fibra para la compensación por dispersión y la modulación en banda lateral única [Smi98]. Por otro lado, la no-linealidad de los amplificadores EDFA ópticos, supone un problema en sistemas de gran densidad de portadoras ópticas (DWDM, dense-WDM), con separación de $50 \mathrm{GHz}$ entre las distintas longitudes de onda. Debido a esto, es necesario introducir filtros ópticos que ecualicen la ganancia de amplificación de los EDFA. Otra opción es el uso de bandas superiores en longitud de onda, como la banda L, 1560-1610 nm, para amplificar longitudes de onda.

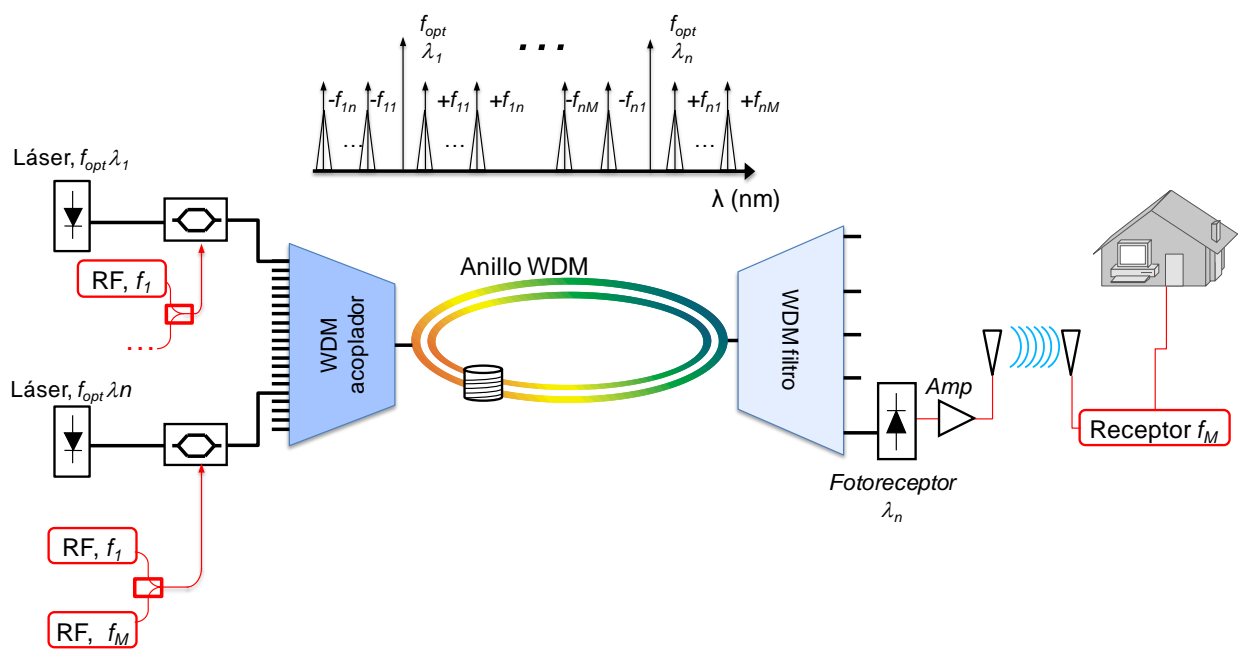

Figura 2.6.Sistema RoF con transporte óptico WDM.

Las redes de acceso basadas en RoF se basan en sistemas y dispositivos que permiten transportar de forma óptica las señales de milimétricas para posteriormente radiarlas, por lo tanto están comprendidas en el área de estudio de las comunicaciones ópticas denominada fotónica de microondas, microwave photonics [See06]. Los elementos clave en estos sistemas son los láseres que generan las portadoras ópticas, los moduladores, los amplificadores ópticos y los foto-detectores capaces de resolver la conversión electro-óptica de estas altas frecuencias de RF, tal y como se puede observar en el sistema RoF con WDM representado en la Figura 2.6.

En las últimas décadas, el desarrollo de los láseres ha sido muy importante para la evolución de los sistemas WDM. Actualmente, en los sistemas RoF se utilizan láseres de diodo tipo DFB (DFB, Distributed Feedback Laser), debido a su ancho de línea estrecho, su bajo ruido y alta linealidad. El uso de los láseres de cavidad vertical (VCSEL, Vertical-Cavity Surface-Emitting Laser) para modulación directa en sistemas RoF se ha incrementado debido a factores como su bajo coste y a su capacidad de transmitir en ventanas adecuadas para sistemas de fibras multimodo, primera ventana y 
segunda ventana de transmisión [Car02]. En cuanto a los moduladores externos, nos encontramos con moduladores basados en el efecto electro-óptico (EOM, Electro-Optical Modulator), compuestos por materiales como el niobato de litio, $\mathrm{LiNbO}_{3}$, o el arseniuro de galio, AsGa, que permiten realizar la conversión de frecuencias eléctricas a ópticas. Actualmente, se trabaja con moduladores con anchos de banda eléctricos superiores a $40 \mathrm{GHz}$ y también con materiales como los polímeros, con el objetivo de reducir la potencia consumida y realizar una conversión electro-óptica mucho más eficiente [Rab02]. Cabe destacar el uso de moduladores de electro-absorción (EAM, Electro-Absorption Modulator) con ancho de banda de trabajo de hasta $40 \mathrm{GHz}$ que permiten una alta integración con fuentes láser [Kaw01]. Estos esquemas de modulación en intensidad y detección directa son los más habituales en los sistemas de RoF y de comunicaciones ópticas, aunque también existen esquemas de tipo coherente [Joh01], [Kah06], de uso más limitado debido a su complejidad. Los foto-detectores actuales son capaces de trabajar con anchos de banda de varios cientos de GHz, y presentan una alta eficiencia de conversión opto-eléctrica [Kaw02].

Los sistemas RoF se consideran adecuados para el transporte y control de señales radio mediante fibra óptica, lo cual permite un grado de flexibilidad y una relación coste-eficiencia muy alta, que trasladada a los diferentes escenarios de aplicación implica importantes beneficios como se muestra en la Tabla 2.2.

TABLA 2.2. VentAJAS DE LOS SISTEMAS ROF EN LAS REDES DE ACCESO

\begin{tabular}{l|l}
\hline \hline \multicolumn{1}{c|}{ Aspectos técnicos } & \multicolumn{1}{c}{ Aspectos económicos y sociales } \\
\hline $\begin{array}{l}\text { Infraestructura versátil } \\
\begin{array}{l}\text { Flexibilidad } \\
\text { Alta relación coste eficiencia }\end{array}\end{array}$ & $\begin{array}{l}\text { Escenarios de oficinas y hogares; uso de } \\
\text { instalaciones previas } \\
\text { Seguridad y privacidad en las redes } \\
\text { Oportunidad de negocio para PYMES }\end{array}$ \\
\hline $\begin{array}{l}\text { Antenas distribuidas, menor potencia de } \\
\text { radiación }\end{array}$ & Uso hospitalario y adecuado a normas sanitarias \\
\hline $\begin{array}{l}\text { CRRM } \\
\text { Movilidad transparente al usuario }\end{array}$ & $\begin{array}{l}\text { Reutilización del ancho de banda inalámbrico de } \\
\text { acceso bajo demanda } \\
\text { Conexión inalámbrica ubicua y transparente }\end{array}$ \\
\hline $\begin{array}{l}\text { Sencilla actualización y bajo coste de } \\
\text { mantenimiento }\end{array}$ & $\begin{array}{l}\text { Recuperación rápida de la inversión, OPEX } \\
\text { Alta fiabilidad a corto y largo plazo }\end{array}$ \\
\hline \hline
\end{tabular}

En el caso de sistemas DAS, los sistemas RoF permiten centralizar muchos procesos en una estación central o BS, lo cual supone reducir la complejidad de las antenas o transceptores, flexibilizando el redimensionamiento y actualización del sistema. Otro de los motivos que ha facilitado la implantación de las redes basadas en sistemas RoF como redes de acceso ha sido la posibilidad de reusar la preinstalación 
existente de fibra multimodo (MMF, Multi-Mode Fibre) en entornos de oficinas e interiores, in-building, para redes de área local. Las redes MMF presentan un coste menor de mantenimiento debido a su amplio diámetro de núcleo, lo cual facilita la reparación de la fibra, reduciendo la complejidad y el coste asociado al despliegue de estas redes [Koo08]. Por último, los sistemas RoF permiten la centralización de procesos en estaciones base, lo que aumenta la eficiencia de la red de acceso al permitir implementar técnicas de procesado de señal como smart antennas o técnicas MIMO (Multiple-Input Multiple-Output). Otra de las ventajas de esta centralización de procesos es la posibilidad de gestionar de forma conjunta los recursos radio disponibles (CRRM, Common Radio Resource Management), como son las técnicas dinámicas de gestión del espectro. Una última ventaja es la implementación de algoritmos para la gestión de la movilidad de los usuarios de forma transparente a los servicios prestados para facilitar la conectividad inalámbrica.

\subsection{Técnicas de integración inalámbrica}

El trabajo presentado en esta Tesis Doctoral evalúa la coexistencia y la integración de las tecnologías de comunicaciones inalámbricas en las redes de acceso actuales, concretamente en los sistemas RoF.

La demanda de servicios de mayor régimen binario por parte de los usuarios finales conlleva aumentar la capacidad en cada celda de cobertura, lo cual implica una reducción de la cobertura inalámbrica de la misma. Un ejemplo lo tenemos en la creación de pico-celdas o sistemas pico-celulares inalámbricos gracias al uso de nuevas frecuencias del orden de las microondas [Sau07]. Por ejemplo, el estándar Wi-Fi IEEE 802.11g para redes inalámbricas WLAN permite regímenes binarios de hasta $54 \mathrm{Mbit} / \mathrm{s}$ en frecuencias del orden de $2.4 \mathrm{GHz}$ [IEEE80211g]. El estándar de acceso inalámbrico mediante microondas WiMAX IEEE 802.16 en su versión inicial propone regímenes binarios de hasta $100 \mathrm{Mbit} / \mathrm{s}$ para rangos de frecuencias entre $10 \mathrm{GHz}$ y $66 \mathrm{GHz}$. Las tecnologías de banda ultra-ancha como UWB de tipo impulsivo permiten regímenes binarios de hasta $480 \mathrm{Mbit} / \mathrm{s}$ en frecuencias en torno a los $24 \mathrm{GHz}$ y los $60 \mathrm{GHz}$. Estos ejemplos indican que para conseguir mayores regímenes binarios los estándares emplean mayores frecuencias, lo que se traduce en celdas de cobertura inalámbrica de menor radio, y la necesidad de aumentar el número de antenas transmisoras para poder dar servicio en la misma área de cobertura. La utilización de sistemas RoF permite implementar estas soluciones en escenarios como oficinas y grandes espacios interiores, centralizando procesos comunes a todas las antenas. En este tipo de sistemas, la generación y el procesado de señales RF se realiza de forma centralizada y se distribuye posteriormente a las antenas mediante el conexionado con fibra óptica. La utilización de sistemas RoF facilita la operatividad, el mantenimiento y la actualización de las redes de acceso. 
Los sistemas RoF se pueden clasificar en función del tipo de fibra óptica utilizado. Los sistemas RoF que usan fibra SMF (RoSMF, Radio-over-Single-Mode-Fibre) se integran mayoritariamente en redes de acceso inalámbricas fijas en exteriores, en las cuales la transmisión en el último tramo hacia el terminal de usuario se realiza vía radio. Entre otros ejemplos, este tipo de sistemas RoSMF se implementa en sistemas como WiMAX o LMDS. El uso de redes basadas en sistemas RoF con fibra MMF (RoMMF, Radio-over-Multi-Mode-Fibre) se contempla para escenarios de acceso inalámbrico interiores que requieren una gran flexibilidad en la gestión de redes locales. Por otro lado, en ambos tipos de sistemas RoF es necesario tener en cuenta las limitaciones impuestas por la utilización de la fibra óptica como son la atenuación, la dispersión cromática o los dispositivos de conversión electro-óptica y opto-eléctrica asociados a cada tipo de fibra.

\subsubsection{Sistemas pico-celulares basados en radio-sobre-fibra}

Un escenario de aplicación de las redes basadas en sistemas RoF son los sistemas pico-celulares. En estos sistemas, es posible distribuir y ofrecer cobertura inalámbrica a través de sistemas de antenas distribuidas (DAN, Distributed Antenna Network). Este tipo de sistemas proporciona conectividad y soporta las funcionalidades de traspaso de usuarios (hand-over) entre pico-celdas, posibilitando la movilidad del usuario a lo largo de toda el área de cobertura.

En la actualidad, este tipo de sistemas son emergentes y se encuentran bajo estudio, debido a la disponibilidad de diversas tecnologías inalámbricas que permiten un elevado régimen binario, lo cual permite diferentes configuraciones. Algunas de las aplicaciones de los sistemas pico-celulares son las comunicaciones para el intercambio de ficheros entre máquinas, la gestión de inventarios en almacenes, la seguridad en edificios o la interconexión a elevados regímenes binarios de dispositivos de forma inalámbrica en redes de área local en distancias cortas. Las redes de topología pico-celular se consideran tanto para la distribución de señales de frecuencias de milimétricas en bandas libres, como son las bandas de frecuencias de 40 o $60 \mathrm{GHz}$ para redes WLAN [Sto99], [Yu06], como para la distribución de señales de tecnologías inalámbricas estandarizadas como WLAN IEEE 802.11.

En la Figura 2.7 se presenta un esquema de arquitectura de red de usuario RoF basada en topología pico-celular para gestionar la cobertura en escenarios interiores. En este caso, se observa cómo cada pico-celda proporciona servicio a varios usuarios, y cómo la inteligencia de la red se concentra en un nodo central (CS, Central Station), lo cual permite tener varias redes de acceso pico-celulares gestionadas bajo el mismo nodo. Esto supone una mejor relación coste-eficiencia para los dispositivos usados en las mismas. Sin embargo, las antenas o interfaces radio constituyen la mayor desventaja 
en el desarrollo de estos sistemas, puesto que deben realizar la conversión E/O y viceversa y estas funciones elevan el coste de los mismos. Sin embargo, la introducción de productos comerciales con producción a gran escala disminuiría el coste efectivo de los mismos.

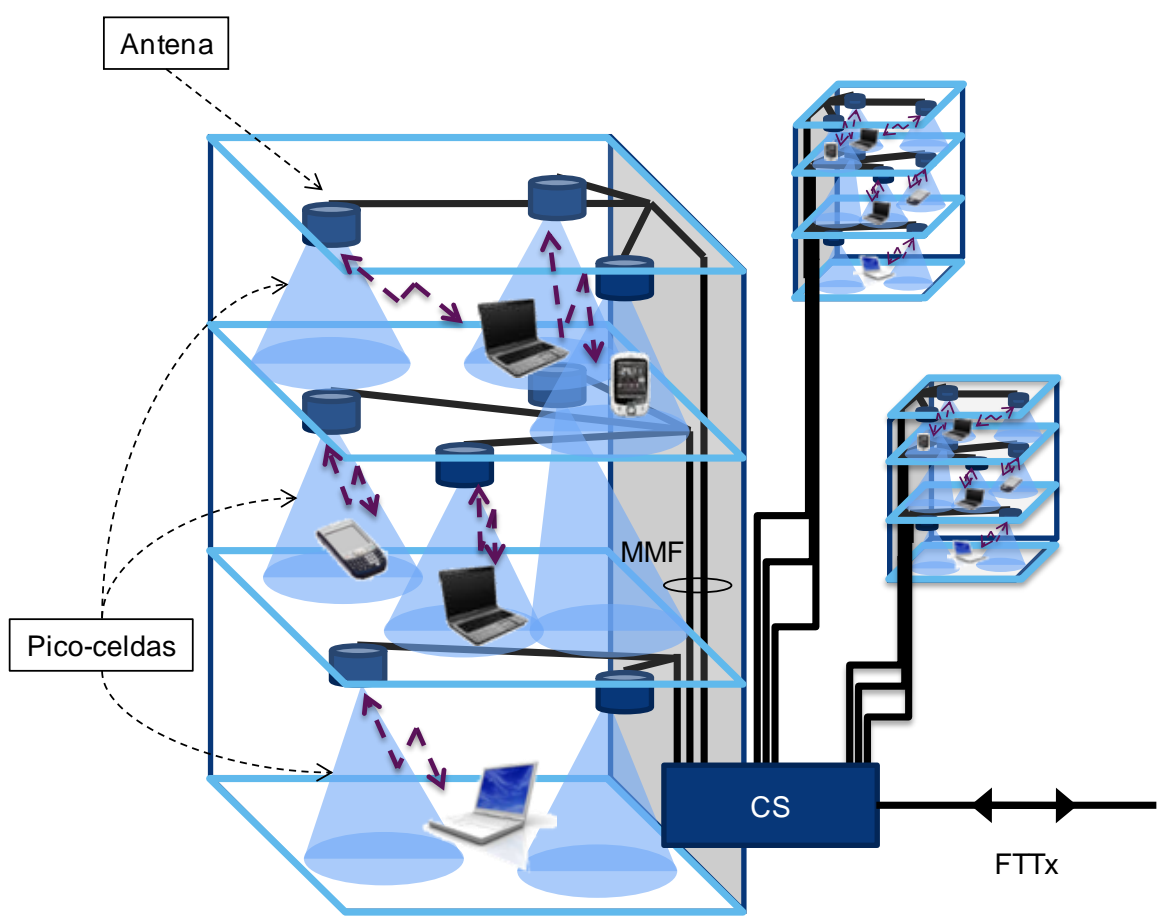

Figura 2.7.Arquitectura de una red de usuario basada en sistemas RoF con topología pico-celular de antenas distribuidas.

El reto que se plantea en las redes basadas en sistemas RoF es la distribución simultánea de varias tecnologías inalámbricas concurrentes en un mismo espacio, como es el caso de sistemas WLAN 802.11x, UWB, GSM o UMTS. Dentro de este tipo de tecnologías, la Voz sobre IP (VoIP, Voice-over-IP) es un servicio que muestra las posibilidades de los sistemas RoF pico-celulares, puesto que permite abaratar el coste tradicional de las llamadas equipo a equipo o equipo a teléfonos, y resulta de gran interés tanto en el entorno empresarial como en el residencial. Para un despliegue efectivo de esta cobertura multiservicio y multioperador en entornos interiores, la tecnología de los dispositivos RoF debe ser más práctica, sencilla y de menor coste. 


\subsection{Tecnologías radiofrecuencia disponibles comercialmente}

Actualmente la demanda de nuevos servicios móviles avanzados ubicuos y capaces de proporcionar un alto régimen binario está impulsando el desarrollo de nuevas tecnologías inalámbricas. Entre estos servicios nos encontramos con la distribución de vídeo de alta definición (HDTV), el acceso a contenidos de Internet de tipo streaming de vídeo y audio, el uso de aplicaciones de comunicaciones audio/vídeo basadas en IP o la gestión de la seguridad de entornos interiores. Estos servicios presentan requisitos comunes en cuanto a elevado régimen binario, movilidad, ubicuidad y disponibilidad universal de acceso radio.

Para satisfacer estas demandas, surgen nuevas tecnologías radio, presentes en múltiples escenarios de aplicación, desde en entornos personales y locales hasta en las redes metropolitanas. La Tabla 2.3 muestra las tecnologías radio disponibles comercialmente según el escenario de aplicación objetivo y su régimen binario máximo. Es importante destacar que la coexistencia en un mismo espacio físico de las tecnologías mostradas en esta tabla es imprescindible para la convergencia en la red de acceso, a fin de proporcionar conectividad en los dispositivos actuales, por ejemplo en ordenadores portátiles que combinan acceso Wi-Fi, Bluetooth y WiMAX [LEN09].

TABLA 2.3. TECNOLOGÍAS DE COMUNICACIONES INALÁMBRICAS EN ENTORNOS DE CORTO Y MEDIO ALCANCE

\begin{tabular}{c|c|c|c}
\hline \hline Área inalámbrica & Tecnología inalámbrica & Estándar IEEE & Régimen Binario \\
\hline \multirow{3}{*}{ WPAN } & Bluetooth & IEEE 802.15 .1 & $3 \mathrm{Mbit} / \mathrm{s}$ \\
\cline { 2 - 4 } & ZigBee & IEEE 802.15 .4 & $250 \mathrm{kbit} / \mathrm{s}$ \\
\cline { 2 - 4 } & UWB & IEEE 802.15 .3 & $2 \mathrm{Gbit} / \mathrm{s}$ \\
\hline WLAN & Wi-Fi & IEEE 802.11 & $54 \mathrm{Mbit} / \mathrm{s}$ \\
\hline WMAN & WiMAX & IEEE 802.16 & $1 \mathrm{Gbit} / \mathrm{s}$ \\
\hline \hline
\end{tabular}

En la Figura 2.8 se muestra la canalización y organización del espacio radioeléctrico para las tecnologías de comunicaciones inalámbricas disponibles comercialmente por debajo de $10.6 \mathrm{GHz}$, en particular Wi-Fi, WiMAX, UWB, Bluetooth, HSPA, 3GPP, GPS, WCDMA, GPRS, EDGE y Mobile TV. En la misma figura se observa la potencia máxima de transmisión para cada una de las tecnologías radio mostradas, según las normas regulatorias de radiocomunicaciones a nivel mundial definidas por diferentes entes regulatorios, como la FCC norteamericana [FCC], la ARIB japonesa [ARIB] o la ETSI en el ámbito europeo [ETSI]. Cabe destacar que la potencia máxima de transmisión en la banda de frecuencias para UWB considerada en esta figura viene definida por la regulación [FCC02.48] 


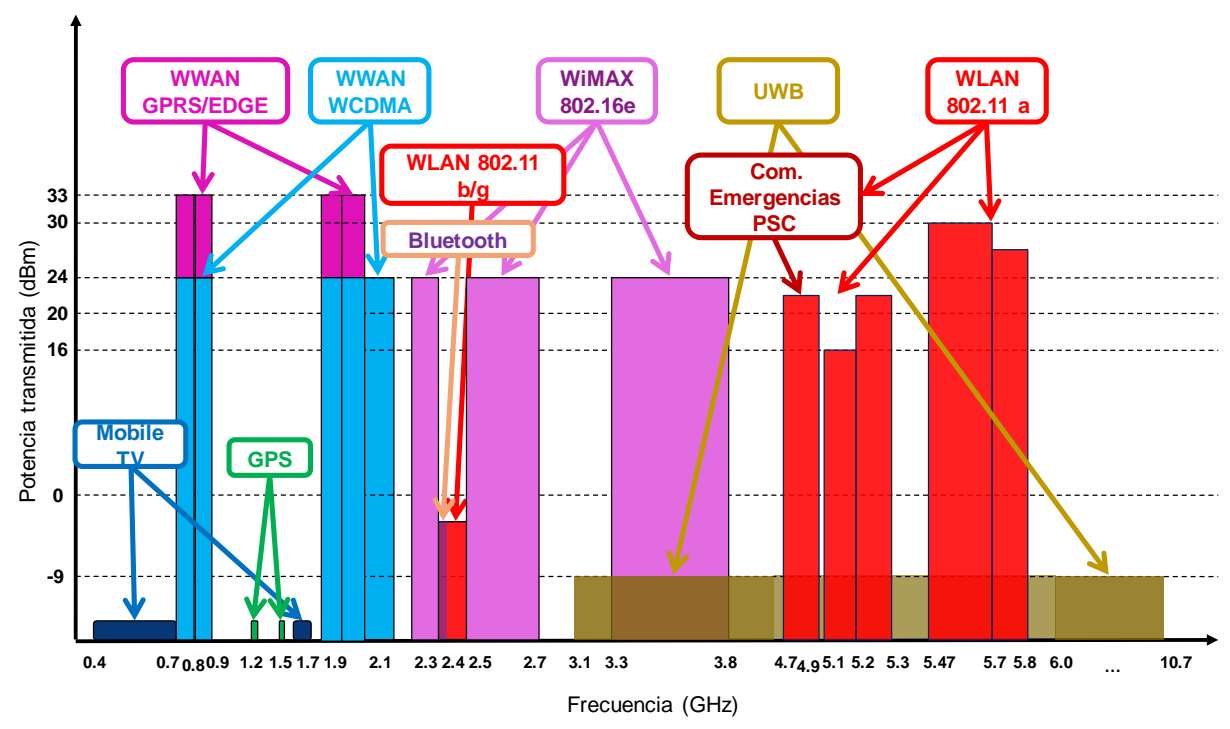

Figura 2.8. Frecuencias de trabajo y potencia transmitida de diversas tecnologías inalámbricas por debajo de $10.6 \mathrm{GHz}$.

Como se puede observar en la Figura 2.8, las comunicaciones WPAN, como Bluetooth, ZigBee y UWB, se solapan en el espectro de radiofrecuencia con comunicaciones WLAN tipo IEEE 802.11, y también con comunicaciones WWAN como WiMAX.

El despliegue de un sistema de comunicaciones WPAN supone contar con la presencia de muchos puntos o sistemas radiantes debido a la existencia de muchos usuarios de la misma tecnología radio en un espacio físico limitado, como un entorno de oficina. Si todos los usuarios funcionaran a la máxima potencia de transmisión regulada, se provocarían problemas reales de interferencia inalámbrica. La investigación sobre la coexistencia entre sistemas inalámbricos es un asunto esencial para el despliegue y desarrollo de cualquier tecnología inalámbrica. Por ello, los estudios de coexistencia en entornos de corto y medio alcance para escenarios convencionales son importantes y necesarios. Este tipo de estudios de investigación de la coexistencia facilitan el despliegue adecuado de comunicaciones radio emergentes como, por ejemplo, la coexistencia entre UWB y sistemas GPS, UMTS, DCS 1800 [Giu05] y televisión digital por satélite (DTV, Digital Television) [Cot05] y Bluetooth [Ham03].

Esta Tesis Doctoral se centra en el estudio de la coexistencia de las tecnologías radio UWB y WiMAX. Ambas tecnologías proporcionan servicios de elevado régimen binario en entornos de corto y medio alcance. El estudio de la coexistencia e interferencia entre ambas permite un despliegue eficiente en los entornos personales de 
tipo WPAN. Por ello, a continuación, se exponen las características y funcionalidades de ambos sistemas de comunicaciones.

\subsection{Características técnicas de WiMAX}

La tecnología WiMAX [IEEE80216e] proporciona servicio inalámbrico de elevado régimen binario para usuarios fijos y móviles en cualquier escenario, desde áreas metropolitanas a interiores de edificios. El primer despliegue comercial WiMAX se produce en 2006 en Corea del Sur con el despliegue de servicio WiMAX en $2.3 \mathrm{GHz}$, conocido bajo el nombre WiBRO, en el área metropolitana de Seúl para ofertar servicios de audio y vídeo de alta calidad. En un reciente estudio se estiman en unos 140 millones los potenciales usuarios de WiMAX a nivel mundial para 2012 [WiM08]. El WiMAX Forum [Wim] es un grupo industrial sin ánimo de lucro cuyo objetivo es asegurar la interoperabilidad entre los diferentes productos WiMAX basados en la familia de estándares IEEE 802.16, a través de un proceso de certificación. En este grupo se encuentran agrupados operadores, industrias electrónicas, proveedores de servicios, fabricantes de chips e industria de contenidos. La actividad de este grupo es el eje fundamental del desarrollo actual de WiMAX, puesto que su actividad permite asegurar la compatibilidad de todos los productos comerciales.

WiMAX es una tecnología de acceso inalámbrico que se basa en los estándares proporcionados por el grupo de trabajo IEEE 802.16 que desarrolla el estándar de acceso inalámbrico de elevado régimen binario en redes de área metropolitana o Wireless MAN [IEEE80216a].

La irrupción del estándar WiMAX con características de movilidad o Mobile WiMAX es una respuesta al cambio en el modelo tradicional de redes móviles celulares, buscando aumentar la capacidad y escalabilidad de los sistemas, y crear arquitecturas compatibles con el trafico IP, además de asegurar la transparencia al usuario y el acceso a nuevos servicios basados en Internet. Todo ello permite el desarrollo de una solución completa (e2e, End-to-End) que responda a estas demandas y a las nuevas oportunidades que de ellas se desprenden, como por ejemplo en las futuras recomendaciones en torno comunicaciones móviles $4 \mathrm{G}$, recogidas en IMT-Advance prevista para principios de 2010.

Mobile WiMAX surgió ligado en 2005 al nuevo estándar IEEE 802.16-2005 revisión 1.0, también conocido como IEEE 802.16e, que soporta WiMAX con especificaciones concretas de parámetros de calidad de servicio y movilidad [IEEE80216e], [IEEE80216d]. Paralelamente, WiMAX Forum planteó un perfil de requisitos para Mobile WiMAX basado en este estándar que permitió incluirlo en su proceso de certificación. Todo estos trabajos dieron como resultado la adopción de 
Mobile WiMAX como el $6^{\circ}$ interfaz radio de la familia IMT-2000 de ITU en 2007 [ITUM1457], definido como una tecnología de acceso dúplex OFDM por división en el tiempo (OFDMA-TDD, OFDM Access Time-Division Duplexing) para redes de tipo WMAN. Con ello, WiMAX IEEE 802.16e se convierte en el estándar que permite la convergencia para el acceso de servicios de elevado régimen binario en redes fijas y móviles. A la tecnología WiMAX IEEE 802.16e también se le conoce como acceso de banda ancha por microondas (BWA, Broadband Wireless Access).

La tecnología Mobile WiMAX también permite la implementación de técnicas de procesado MIMO y de conformado de haces o beamforming. Cabe indicar que la revisión 1.0 de WiMAX, basada en IEEE 802.16e, solo definió a TDD como el modo de operación para las bandas de $2.3 \mathrm{GHz}, 2.5 \mathrm{GHz}, 3.5 \mathrm{GHz}$ y $8.75 \mathrm{GHz}$ inicialmente, pero las revisiones realizadas en el seno de WiMAX Forum ya contemplan FDD como formato de operación en la revisión 1.5.

La compresión, el estudio y la pormenorización de la tecnología radio WiMAX IEEE 802.16e queda fuera del objetivo de esta Tesis Doctoral. Sin embargo, es necesario conocer ciertos parámetros importantes a nivel de capa física de transmisión para contextualizarla en el marco de esta Tesis Doctoral.

\subsubsection{Mobile WiMAX}

La principal característica de Mobile WiMAX es el uso de OFDMA como tecnología de acceso. OFDMA proporciona diversidad en frecuencia frente a desvanecimientos multicamino y por lo tanto es adecuado para situaciones sin visión directa (NLOS, Non-Light of Sight). Esto permite la utilización de WiMAX IEEE 802.16e en frecuencias por debajo de $10.66 \mathrm{GHz}$. OFDMA incluye una subcanalización que permite reubicar dinámicamente los recursos temporales y de frecuencia disponibles para cada usuario a través de las subtrama de subida (UL, Uplink) y bajada (DL, Downlink). De esta forma, varios usuarios asignados a diferentes grupos de frecuencias de subportadoras pueden transmitir simultáneamente en el tiempo. Los usuarios son recolocados dinámicamente en la duración de la subtrama tanto en tiempo como en frecuencia. Esta asignación dinámica aumenta la eficiencia de los recursos radio disponible, pero a su vez aumenta la complejidad del control de la capa física [Ste07].

Otra característica de la revisión 1.0 de WiMAX 802.16e es el uso de de TDD como forma de operación en el sistema. Como se especificaba ya en [IEEE80216d], TDD permite el tráfico asimétrico entre DL y UL. El tráfico asimétrico aumenta la reutilización espectral y la eficiencia del sistema en comparación con la operación de división por duplexión en frecuencia (FDD, Frequency-Division Duplexing), que 
requiere de anchos de banda iguales para DL y UL. El uso de un canal común para las transmisiones UL y DL en TDD simplifica el diseño de los transceptores WiMAX. TDD permite la reubicación de recursos radio para soportar tráfico variable, además asegura la reciprocidad en el canal de transmisión, lo cual permite disponer de conocimiento del canal en transmisión y aplicar determinadas técnicas MIMO, especialmente beamforming.

Como se ha comentado anteriormente, la estructura de la trama WiMAX 802.16e es compleja y muy flexible. La duración de una trama WiMAX 803.16e suele ser de unos $5 \mathrm{~ms}$ [IEEE80216e] y se divide en subtramas para DL y UL de duración flexible, separadas entre ambas por pequeños tiempos de transición, denominados TTG y RTG, para evitar colisiones en la transmisión. En la Figura 2.9 se muestra la estructura y canalización de una trama TDD de WiMAX 802.16e. En una trama de WiMAX IEEE 802.16e se definen los siguientes canales físicos:

- Preámbulo (preamble): se transmite siempre en el primer símbolo OFDM de la trama en DL y se usa para sincronización del canal radio entre estación base (BS) y terminal (TS, Terminal Station) y para tareas de reconocimiento.

- Trama de cabecera de control (FCH, Frame Control Header): es continuación al preámbulo y proporciona información de configuración de la trama, como el esquema de codificación o los subcanales usados.

- DL-MAP, UL-MAP: indican información sobre la ubicación de los recursos en las subtramas DL y UL.

- UL ranging: es un subcanal que usan los TS para realizar medidas continuas de tiempo, frecuencia y ajustes de potencia conforme lo exigen los diferentes anchos de banda configurados.

- UL CQICH: es un canal ubicado para que el terminal pueda recibir información de retorno del estado del canal.

- UL ACK: se ubica para que el TS pueda realimentar información útil para el control de la comunicación. 


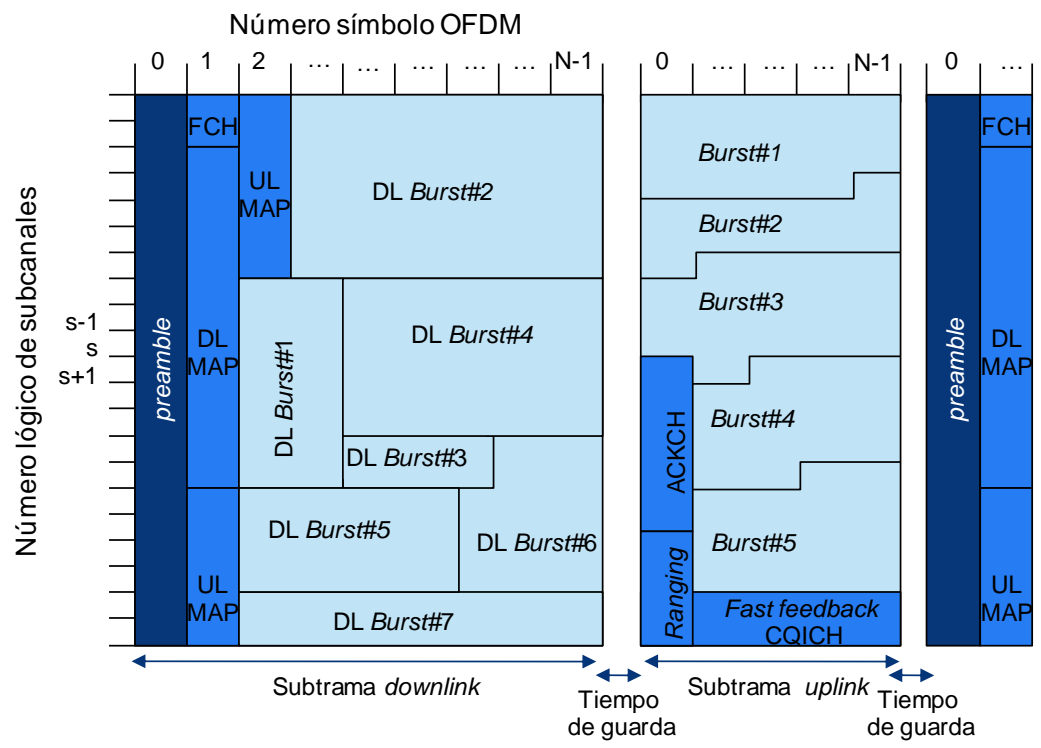

Figura 2.9. Estructura de trama TDD para Mobile WiMAX [IEEE80216e].

En el estándar Mobile WiMAX, la capa radio SOFDMA-PHY (SOFDMA, Scalable OFDM Access) define anchos de banda nominales que varían desde $1.25 \mathrm{MHz}$ hasta $20 \mathrm{MHz}$, en cada caso se utilizan desde 128 hasta 2048 puntos FFT (FFT, Fast-Fourier Transform), permitiendo comunicaciones escalables variando el espaciado entre subportadoras [IEEE80216e]. A su vez, las diferentes combinaciones de modulaciones disponibles y tasas de codificación permiten disponer de un gran abanico de regímenes binarios. Un ejemplo de la escalabilidad SOFDMA que permite WiMAX 802.16e lo tenemos en los parámetros de la Tabla 2.4. En ella se fijan el espaciado entre subportadoras y la duración del símbolo OFDM y se observa cómo se escalan los parámetros de la señal WiMAX dependiendo del ancho de banda nominal y del tamaño de la FFT. Cabe recordar que el número de puntos FFT hace referencia al número de subportadoras usadas en por OFDMA.

Tabla 2.4. Parámetros OFDM escalable Para IEEE 802.16E de 5 MS dE DURACIÓN CON CP DE 1/8

\begin{tabular}{|c|c|c|c|c|c|}
\hline "Ancho de banda nominal (MHz) & 1.25 & 2.5 & 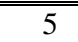 & 10 & 20 \\
\hline Frecuencia de muestreo (MHz) & 1.4 & 2.8 & 5.6 & 11.2 & 22.4 \\
\hline Puntos FFT & 128 & 256 & 512 & 1024 & 2048 \\
\hline Espaciado subportadoras $(\mathrm{kHz})$ & \multicolumn{5}{|c|}{10.94} \\
\hline $\begin{array}{l}\text { Duración de símbolo OFDM }(\mu \mathrm{s}) \\
\left(T_{s}=1 / \text { Espaciado Subportadora) }\right.\end{array}$ & \multicolumn{5}{|c|}{102.86} \\
\hline Tiempo útil símbolo $(\mu \mathrm{s})$ & \multicolumn{5}{|c|}{$91.43 \mu \mathrm{s}$} \\
\hline $\begin{array}{l}\text { Prefijo cíclico }(\mu s) \\
\text { (T. útil símbolo/8) }\end{array}$ & \multicolumn{5}{|c|}{$11.43 \mu \mathrm{s}$} \\
\hline
\end{tabular}


La tecnología WiMAX 802.16e permite disponer de cobertura en áreas de hasta $8.4 \mathrm{~km}$ para anchos de banda nominales de $5 \mathrm{MHz}$ y $10 \mathrm{MHz}$.

Actualmente, Mobile WiMAX se encuentra estandarizado por IEEE 802.16e [IEEE80216e], y también con las revisiones 1.0 y 1.5 realizadas por el WiMAX Forum. Este conjunto de estándares y revisiones completa la definición de la tecnología Mobile WiMAX a nivel de comportamiento de red y parámetros radio. A su vez, se está trabajando en el seno de IEEE 802.16 en la transición desde esta revisión 1 hacia la revisión 2 o futura IEEE $802.16 \mathrm{~m}$, que busca cumplir con los requisitos de IMT-Advance con respecto a la próxima generación de redes móviles prevista para principios de 2010.

La normativa que rige el despliegue de las redes WiMAX por debajo de $10.6 \mathrm{GHz}$ queda reflejada en las diferentes decisiones de los organismos reguladores al respecto. Mención especial merece la regulación europea del 2007 ECC/07/02, que regula el despliegue de las comunicaciones BWA o WiMAX 802.16e en la banda de $3.4 \mathrm{GHz}$ a $3.8 \mathrm{GHz}$ [ECC07.02].

\subsection{Características técnicas de UWB}

La tecnología UWB se caracteriza por su gran ancho de banda espectral. Una señal de RF se define como de banda ultra-ancha cuando su ancho de banda a $-3 \mathrm{~dB}$ de su frecuencia central es como mínimo el $25 \%$ de su ancho de banda total. Las primeras contribuciones en el campo de la tecnología UWB fueron realizadas por el Dr. Ross y el Dr. Bennett [Ben78] y por el Dr. Harmuth [Har81]. Cabe destacar que en 1973 se concedió la primera patente en comunicaciones UWB a los trabajos realizados por el Dr. Ross desde los primeros años 60 en colaboración con el Dr. Robbins y el Dr. Fontana [Ros73]. En los años 70, UWB fue denominada como tecnología de portadora libre (carrierless), banda base o de impulso en banda base. Durante los años 80, la tecnología UWB de pulsos cortos de gran ancho de banda se desarrolló mediante programas militares a nivel mundial, desde EEUU hasta la extinta URSS [Gle84], debido a sus aplicaciones en el ámbito de las comunicaciones militares, de los sistemas radar, de los sistemas de reconocimiento y de otras aplicaciones puntuales. A finales de los años 80 se hicieron públicos los primeros desarrollos de la tecnología UWB de portadora libre, y fue entonces cuando se comenzó a adoptar el término UWB. En 1986 el Dr. Ross y el Dr. Fontana realizaron la primera prueba de campo de un sistema de comunicaciones UWB de pulsos cortos. En 1994, se inventó el radar de impulsos de micro potencia (MIR), que propuso por primera vez un sistema radar UWB que operaba a muy baja potencia con la única alimentación de baterías. Este radar permitía implementar algoritmos de detección muy sensibles a la dirección de llegada [Mce94]. 
Este gran interés en UWB se ve reflejado en el año 2000 cuando la agencia de regulación de las comunicaciones (FCC, Federal Communications Comission) [FCC] de los EEUU propone regular el uso de UWB para usos civiles, bajo la normativa [FCC02.48]. En esta normativa se definen tres casos de uso de los dispositivos UWB para uso civil: (i) radiocomunicaciones y sistemas de medida, (ii) uso en comunicaciones vehiculares tipo radar y (iii) sistemas de reconocimiento o imaging como radares de penetración en tierra o muros, o sistemas de reconocimiento médico y de vigilancia. Con esta normativa, se reconoce a la tecnología UWB como una tecnología civil de grandes aplicaciones. Esta Tesis Doctoral se centra en el uso de UWB como tecnología radio para comunicaciones.

\subsubsection{Principio de operación}

La tecnología de radiocomunicaciones UWB queda enmarcada en su uso comercial para comunicaciones en el espectro radioeléctrico de $3.1 \mathrm{GHz}$ hasta $10.6 \mathrm{GHz}$, según la normativa de EEUU, Europa y de la mayoría de regiones del mundo [ETSI08], [FCC02.48]. Cabe destacar que en esta normativa una señal RF es considerada como de comunicaciones radio UWB cuando su ancho de banda fraccional a $-10 \mathrm{~dB}$ es mayor del $20 \%$ de su frecuencia de trabajo, como mínimo $500 \mathrm{MHz}$ de ancho de banda según la [FCC02.48], o mayor de $50 \mathrm{MHz}$ de ancho de banda a $-23 \mathrm{~dB}$ según la normativa europea [ETSI08]. Al ser considerado como un sistema de radiocomunicaciones sin licencia de banda ultra-ancha, los sistemas UWB deben coexistir en muchos casos con otros sistemas de comunicaciones de banda estrecha con licencia. Esta coexistencia obliga a regular las potencias de emisión y recepción de los sistemas UWB para evitar escenarios de interferencia no deseada. A este respecto los diferentes grupos de trabajo en los organismos regulatorios internacionales, como la FCC, la ETSI o la ARIB japonesa proponen un marco espectral para la regulación de la potencia de emisión UWB en la banda de 3.1 a $10.6 \mathrm{GHz}$ para aplicaciones interiores o de corto alcance. Esta regulación define la densidad de potencia máxima transmitida (EIRP, Equivalent Isotropically Radiated Power) en la banda de interés en $-41.3 \mathrm{dBm} / \mathrm{MHz}$ y también define el máximo valor de potencia de pico en la banda de trabajo como de $0 \mathrm{dBm} / 50 \mathrm{MHz}$.

Las regulaciones de la potencia transmitida según la posición de su banda de frecuencia en el espectro radioeléctrico se conocen como máscaras espectrales de potencia. Como se observa en la Figura 2.10, para UWB se definen diferentes máscaras espectrales de potencia. Se observa como generalmente se adopta el valor de $-41.3 \mathrm{dBm} / \mathrm{MHz}$, aunque éste varía en Europa y Japón en ciertas bandas para proteger sistemas públicos de comunicaciones especiales. Además tanto en Europa como en Japón se proponen técnicas para proteger y minimizar la interferencia de UWB sobre otras tecnologías como WiMAX o servicios de radiolocalización. El uso de estas 
técnicas condiciona en parte la máscara espectral de potencia UWB, como se observa para aquellas que usan técnicas de mitigación de la interferencia del tipo por reducción del ciclo de trabajo (LDC, Low Duty Cicle) y por detección y exclusión o evitación (DAA, Detect-and-Avoid).

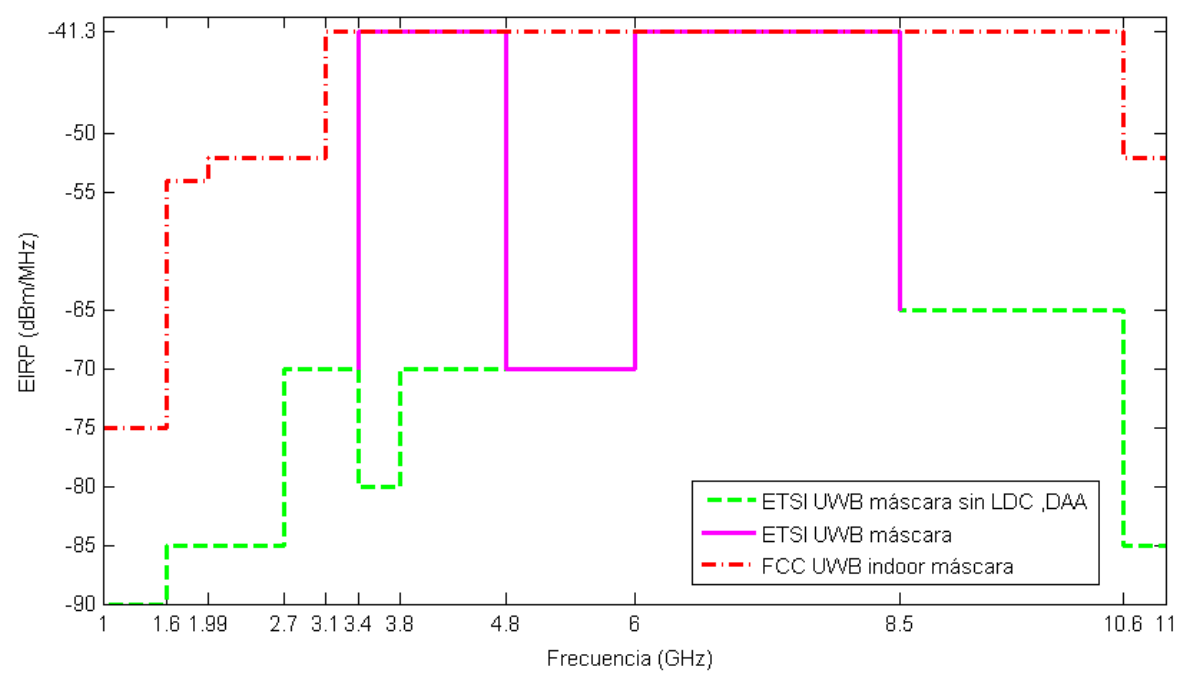

Figura 2.10. Máscara espectral de máxima EIRP para UWB radio según las regulaciones internacionales vigentes.

El nivel de EIRP máximo de $-41.3 \mathrm{dBm} / \mathrm{MHz}$ se especifica a partir de los estudios realizados sobre la relación de potencia transmitida y el alcance para determinados regímenes binarios. Según los estudios de los diferentes entes reguladores, la regulación de potencia mostrada en la Figura 2.10 permite no perjudicar de forma ostensible a sistemas con licencia y también coexistir con otros como IEEE 802.11 en espacios reducidos, donde con esta potencia UWB se pueden realizar comunicaciones con regímenes binarios superiores a los $100 \mathrm{Mbit} / \mathrm{s}$. UWB se define por lo tanto como un sistema de radiocomunicaciones para enlaces radio de corta distancia a muy alta velocidad, o para enlaces de media y larga distancia a muy baja velocidad en aplicaciones de sensado, seguridad o transmisiones sensibles. En el estudio y evaluación experimental presentados en esta Tesis Doctoral se ha considerado a UWB como una tecnología de radiocomunicaciones para enlaces de corta distancia y elevados regímenes binarios.

UWB es una tecnología inalámbrica de gran interés en los entornos académicos e industriales debido a su versatilidad para un gran número de aplicaciones. Por ejemplo, en aplicaciones de corto alcance UWB puede llegar a exhibir hasta $1 \mathrm{Gbit} / \mathrm{s}$ de régimen binario [Lun06], lo que la convierte en perfecta candidata para transmisiones 
inalámbricas de elevados regímenes binarios como son los actuales formatos de entretenimiento audiovisual de alta definición o conexiones de datos inalámbricas a elevado régimen binario en cortas distancias. Otros entornos de implementación UWB surgen en aplicaciones de comunicaciones vehiculares de corta distancia [Pai07], como radares en vehículos, transmisiones coche a coche (C2C, Car-to-Car) o comunicaciones infraestructuras-coche (I2V, Infrastructure-to-Vehicule). Estas aplicaciones utilizan la banda de $22 \mathrm{GHz}$ a $29 \mathrm{GHz}$ bajo la actual normativa UWB [FCC02.48], [ECC04]. Existen otras aplicaciones de tipo radar basadas en tecnología UWB como las realizadas en la banda $\mathrm{V}$ de $\mathrm{RF}$, es decir, por encima de los $65 \mathrm{GHz}$ [Str05], plenamente vigentes aunque fuera del objetivo de esta Tesis Doctoral. El gran auge de UWB viene respaldado por características inherentes a esta tecnología radio como son su inmunidad al desvanecimiento multicamino, su baja probabilidad de intercepción, su baja interferencia entre canales UWB y su capacidad de establecer comunicaciones a través de paredes [Koh04].

En entornos de aplicaciones UWB de corta distancia existen dos implementaciones UWB claramente diferenciadas: aquellas de portadora libre o carrierless y aquellas que usan múltiples portadoras o multi-carrier. Dentro de la tecnología carrierless UWB se encuentran los sistemas denominados de impulsos de radio UWB (IR-UWB, Impulse Radio UWB), puesto que basa su sistema de comunicaciones en la transmisión de pulsos de alta energía y muy corta duración. En los sistemas de tipo IR-UWB, el pulso se sube en frecuencia y su ancho de banda se adecua a la frecuencia de la aplicación correspondiente. Los sistemas de pulsos UWB utilizan diversos formatos de generación de pulsos en sus comunicaciones. Por ejemplo, existen comunicaciones IR-UWB basadas en time-modulated UWB (TM-UWB), que emiten de forma discontinua pulsos ultra cortos o formas de onda pulsadas en base a monociclos. Otro ejemplo son las comunicaciones UWB donde los pulsos se transmiten codificando su fase en secuencias de pulsos denominados direct sequence phase code UWB (DSC-UWB), lo cual permite aumentar la cantidad de información transmitida y mejorar la respuesta frente al desvanecimiento multicamino. Este tipo de sistemas también se conoce como UWB de secuencia directa (DS-UWB) [Con99]. Por último, tenemos los sistemas denominados fast stepped frequency chirps UWB (FC-UWB). En este caso, los sistemas generan una señal que cumple con los parámetros UWB mediante un barrido del oscilador local en frecuencia. Este tipo de sistemas es habitual en radares de penetración (GPR, Ground Penetration Radar) para detección de minas, rescate de personas y objetos sepultados entre otros [Sti97], [Cor95].

Por otra parte, las tecnologías multiportadora UWB disponen de canales divididos en bandas de mínimo $500 \mathrm{MHz}$, esta división permite disponer de un acceso múltiple y aumentar la capacidad de los sistemas UWB. Para implementar este acceso se utilizan varias portadoras. El acceso múltiple para multiportadora UWB se realiza mediante 
multiplexación en código, como CDMA, o mediante el acceso por multiplexación en frecuencias ortogonales, OFDM. Los sistemas de acceso por código son aquellos que mediante secuencia directa, espectro ensanchado y el uso de varias portadoras son capaces de abarcar todo el espectro UWB disponible. Por ejemplo, los sistemas DS-CDMA, MC-CDMA y multi-tone MT-CDMA UWB [Pra96]. La propuesta de acceso multiportadora basada en portadoras ortogonales y saltos de frecuencia en su acceso y transmisión se conoce como multibanda OFDM UWB (MB-OFDM UWB, Multi-Band OFDM UWB) [Bat03]. MB-OFDM UWB propone una canalización del espectro UWB disponible en 14 subcanales de $528 \mathrm{MHz}$, agrupados a su vez en 6 canales. Entre sus características cabe destacar el ensanchamiento espectral que supone el uso de 128 subportadoras OFDM por cada subcanal UWB y la codificación de la transmisión para realizar saltos entre frecuencias en el tiempo para permitir una mayor robustez frente al desvanecimiento multicamino. En la Figura 2.11 se muestra un esquema de las diferentes tecnologías UWB desarrolladas hasta la actualidad.

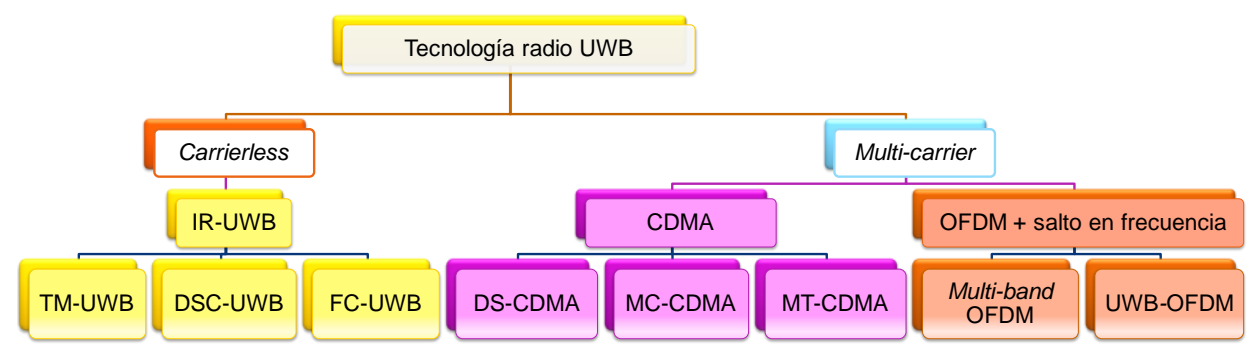

Figura 2.11. Implementaciones de la tecnología UWB.

\subsubsection{Sistemas basados en impulse-radio}

La tecnología IR-UWB se basa en la transmisión de pulsos de duración muy corta. La transmisión de estos pulsos no requiere de una portadora, ya que debido a la duración del pulso se consigue adecuar su transmisión al ancho de banda deseado sin canalizaciones previas.

Los primeros sistemas de generación de señales IR-UWB se desarrollaron a principios de los años 70. Habitualmente la generación de pulsos IR-UWB se basa en monociclos. Existen múltiples técnicas de generación de pulsos IR-UWB, entre las que cabe destacar aquellas de tipo fotónico. Por ejemplo, en [Kur06] se propone un sistema de generación que emplea la supresión de portadora residual, lo cual permite adecuar la máscara espectral UWB del espectro subido en frecuencia gracias a pulsos rectangulares. Otras técnicas utilizan moduladores electro-ópticos $\mathrm{MZ}$ funcionando en la zona no lineal para subir en frecuencia monociclos gaussianos o pulsos doublet, generados mediante transmisores eléctricos comerciales [Leg07]. Por otra parte, la generación óptica de pulsos también se puede realizar mediante el uso de moduladores 
MZ-EOM duales en paralelo con señales eléctricas gaussianas [Cha08b]. Existen técnicas de generación de pulsos que consideran sistemas de láser pulsado junto a MZ-EOM y foto-detectores balanceados [Bel08]. Otras estructuras más complejas para la generación de pulsos IR-UWB comprenden la subida en frecuencia de los monociclos mediante la rotación no lineal de la polarización presente en los amplificadores ópticos de semiconductor. En estas propuestas los monociclos se generan usando pulsos eléctricos gaussianos y discriminadores de frecuencia [Fu08]. Cabe destacar las técnicas de generación de pulsos IR-UWB en la banda de frecuencias de milimétricas, por ejemplo para su aplicación en la banda de $60 \mathrm{GHz}$ [Bel09]. Esta banda de frecuencias se está considerando para futuras comunicaciones radio de elevado régimen binario [Sno07].

Por otra parte, una ventaja inherente a la generación óptica de señales IR-UWB es la detección fotónica que reduce la interferencia con otros sistemas [Kur06], [Leg07].

Actualmente IR-UWB es una tecnología de gran interés para aplicaciones de corto alcance, como comunicaciones exteriores del tipo C2C y I2V en vehículos [Pai06], y para radares de reconocimiento de corto alcance y alta resolución [Str05], por ejemplo radares de alcance de hasta $10 \mathrm{~m}$ en $24 \mathrm{GHz}$ [Mei05]. Las técnicas de generación IR-UWB muestran que es una tecnología con gran aplicación, tanto para las futuras bandas de $60 \mathrm{GHz}$, como para comunicaciones vehiculares y aplicaciones radar en $24 \mathrm{GHz}$.

El estudio y la evaluación experimental mostrados en esta Tesis Doctoral se han basado en la implementación de la tecnología UWB radio basada en MB-OFDM. La compresión, el estudio y la pormenorización de la tecnología MB-OFDM UWB queda fuera del objetivo de esta Tesis Doctoral. Sin embargo, es necesario conocer con mayor profundidad ciertos parámetros que definen a esta tecnología.

\subsubsection{Sistemas basados en multi-band OFDM}

La tecnología de multiportadora basada en OFDM, para comunicaciones UWB de elevado régimen binario y entornos de corto alcance se promovió inicialmente en el seno del consorcio industrial Multi-band OFDM Alliance (MBOA), bajo el amparo de Intel, Sony y Alereon. Posteriormente, en el año 2005, este consorcio se unió al grupo de interés WiMedia Alliance junto con HP, Samsung y Texas Instruments con el objetivo de fomentar el uso y el desarrollo eficaz de esta tecnología UWB. Al mismo tiempo que MBOA comenzaba sus actividades en torno a MB-OFDM UWB, el grupo de interés $U W B$ Forum, formado por Motorola y Freescale, se fundó para el fomento de la tecnología UWB basada en DS-UWB, como soporte radio para dispositivos de comunicaciones de elevado régimen binario y corto alcance. 
Esta confrontación industrial en cuanto al sistema o tecnología UWB más adecuado para entornos WPAN ha tenido su reflejo en el proceso de estandarización, que ha llevado a cabo el grupo de trabajo del IEEE para escenarios de comunicaciones inalámbricas de elevado régimen binario para entornos personales, es decir, el IEEE 802.15 TG3a. Este grupo de trabajo TG3a debía elegir la tecnología de base radio UWB para comunicaciones WPAN de elevado régimen binario (PHY High Rate) pero se disolvió sin decidir una estandarización concreta. Actualmente, su estandarización a nivel físico está supeditada a los trabajos que el grupo de acceso al medio TG3b en el seno de IEEE 802.15 está llevando a cabo. El futuro del estándar IEEE 802.15.3 se decidirá en base a las conclusiones de los estudios realizados por el grupo TG3b y de la penetración comercial de las tecnologías UWB. Cabe destacar que DS-UWB se ha adoptado como tecnología radio en el grupo de trabajo para entornos WPAN de baja régimen binario, IEEE 802.13 TG4a. Este grupo lleva a cabo la estandarización para conseguir un formato de tecnología radio que presente gran ancho de banda, menor consumo, y bajo régimen binario dirigido a aplicaciones de reconocimiento, posicionamiento y control

Tras acabar los trabajos de TG3a, el consorcio WiMedia-MBOA recogió las contribuciones UWB de nivel físico (PHY UWB) que se habían realizado y las presentó a la asociación internacional ECMA. Ésta aceptó la propuesta y publicó a finales de 2005 un estándar para fabricación y posterior desarrollo de componentes basado en MB-OFDM UWB. Este estándar técnico es el ECMA-368 [ECMA368] que incorpora la capa física radio de la tecnología MB-OFDM UWB y también detalles sobre la capa de acceso al medio (MAC, Medium Access Layer). En este estándar se plantea la utilización de MB-OFDM UWB para aplicaciones de localización (ranging) y para comunicaciones radio de elevado régimen binario. Además, se lanzó conjuntamente la especificación ECMA-369 [ECMA369], en la cual se desarrolla la interfaz hardware MAC-PHY para MB-OFDM UWB. En el año 2007, estas especificaciones fueron reconocidas como estándares ISO. Esto ha supuesto la adopción definitiva de MB-OFDM UWB como tecnología para WPAN de elevado régimen binario. Las especificaciones que cumplen con la normativa ISO/IEC son:

i) ISO/IEC 26907:2007 - Information technology - Telecommunications and information exchange between systems - High Rate Ultra Wideband PHY and MAC Standard [ISO26907].

ii) ISO/IEC 26908:2007 - Information technology - MAC-PHY Interface for ISO/IEC 26907 [ISO26908].

La especificación completa de MB-OFDM UWB ha permitido disponer de una tecnología UWB que conlleva una capa radio, PHY, y una de interfaz común de acceso 
al medio, MAC-PHY. Esto permite a los grupos de interés que desarrollan aplicaciones de corto alcance con elevados regímenes binarios, la opción de elegir la plataforma radio MB-OFDM UWB como base para sus futuros desarrollos, como se muestra en la Figura 2.12. En el año 2009, el grupo de interés especial WiMedia Alliance anunció sus planes de realizar la transferencia completa de su tecnología al grupo de fabricantes de USB (USB-IF, USB Implementers Forum) para el desarrollo de Wireless USB [USB09] y también al grupo de interés Bluetooth para el desarrollo del Bluetooth 3.0 [BLU09]. Esto ha supuesto el cese inmediato de las actividades de WiMedia Alliance. Se espera que con este traspaso tecnológico, MB-OFDM UWB encuentre el apoyo tecnológico, industrial y comercial necesario para convertirse en un estándar radio de facto para comunicaciones de corto alcance y elevados regímenes binarios.

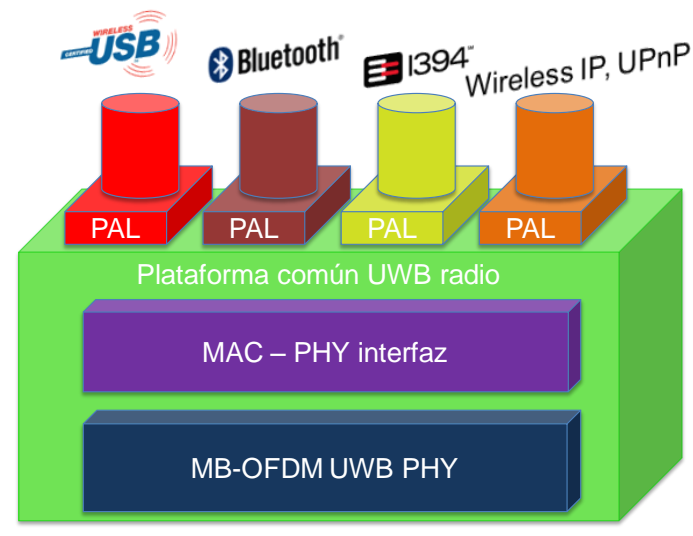

Figura 2.12. Plataforma MB-OFDM UWB radio y sus aplicaciones actuales y futuras. PAL: Protocol Adaptation Layer, capa para la adaptación de protocolos.

Tras haber mostrado la regulación de la tecnología MB-OFDM UWB, también llamada WiMedia-defined UWB, se exponen a continuación sus especificaciones radio. Estas especificaciones han sido definidas en el estándar ECMA-368 [ECMA368].

\subsubsection{Especificaciones radio MB-OFDM UWB}

La máscara espectral de potencia MB-OFDM UWB está situada en la banda de frecuencias entre $3.1 \mathrm{GHz}$ y $10.6 \mathrm{GHz}$, y proporciona 14 canales o bandas de $528 \mathrm{MHz}$ de ancho de banda cada uno [FCC02.48], [ECMA368]. Las bandas se agrupan a su vez en 6 grupos de bandas (BG, Band Group), donde el BG5 solo tiene 2 bandas y el BG6 comparte las bandas de BG3 y BG4. 


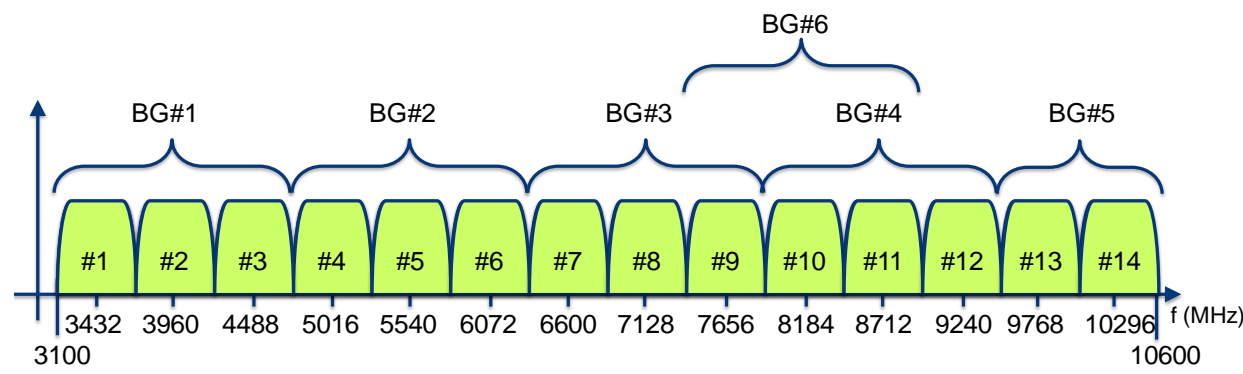

Figura 2.13. Plan de frecuencias MB-OFDM UWB.

La Figura 2.13 muestra el plan de frecuencias MB-OFDM UWB. Se define como banda obligatoria (mandatory) para comunicaciones MB-OFDM UWB a aquellos canales presentes por debajo de la frecuencia $5 \mathrm{GHz}$, en este caso la banda BG\#1 formada por las subbandas $\{1,2,3\}$. La banda BG1 se ha definido como mandatory debido a que este grupo de canales es más robusto frente a los desvanecimientos multicamino [Bat03]. En la Tabla 2.5 se presenta de forma detallada el plan de frecuencias para MB-OFDM UWB mostrado en la Figura 2.13.

Tabla 2.5. Plan Detallado de fRecuencias EN MB-OFDM UWB

\begin{tabular}{c|c|c|c|c}
\hline \hline Banda BG & Banda_ID $\left.\mathbf{( n}_{\mathbf{b}}\right)$ & $\mathbf{f}_{\text {inferior }}(\mathbf{M H z})$ & $\mathbf{f}_{\text {central }}(\mathbf{M H z})$ & $\mathbf{f}_{\text {superior }}(\mathbf{M H z})$ \\
\hline \multirow{3}{*}{1} & 1 & 3.168 & 3.432 & 3.696 \\
\cline { 2 - 5 } & 2 & 3.696 & 3.960 & 4.224 \\
\cline { 2 - 5 } & 3 & 4.224 & 4.488 & 4.752 \\
\hline \multirow{3}{*}{3} & 4 & 4.752 & 5.016 & 5.280 \\
\cline { 2 - 5 } & 5 & 5.280 & 5.544 & 5.808 \\
\cline { 2 - 5 } & 6 & 5.808 & 6.072 & 6.336 \\
\cline { 2 - 5 } & 7 & 6.336 & 6.600 & 6.864 \\
\cline { 2 - 5 } & 8 & 6.864 & 7.128 & 7.392 \\
\hline \multirow{3}{*}{4} & 9 & 7.392 & 7.656 & 7.920 \\
\cline { 2 - 5 } & 10 & 7.920 & 8.184 & 8.448 \\
\cline { 2 - 5 } & 11 & 8.448 & 8.712 & 8.976 \\
\cline { 2 - 5 } 5 & 13 & 8.976 & 9.240 & 9.504 \\
\cline { 2 - 5 } & 14 & 9.504 & 9.768 & 10.032 \\
\cline { 2 - 5 } & 9 & 10.032 & 10.296 & 10.560 \\
\cline { 2 - 5 } & 10 & 7.392 & 7.656 & 7.920 \\
\hline \hline
\end{tabular}

La canalización del sistema MB-OFDM UWB se basa en un conjunto de códigos de tiempo-frecuencia (TFC, Time-Frequency Codes) que codifican la frecuencia central donde se transmite cada símbolo OFDM. Cada TFC indica la codificación para 6 símbolos OFDM (OFDM burst). Los grupos BG1 al BG4 y el BG6, al estar 
compuestos por 3 bandas cada uno, tienen los mismos códigos TFC pero usando sus bandas asignadas. Por ejemplo, TFC1 en BG1 se codifica mediante la secuencia de salto entre bandas 1-2-3-1-2-3, para BG4 este código se codifica mediante la secuencia 10-11-12-10-11-12 y para BG6 este código se codifica mediante la secuencia 9-10-11-9-10-11. Cabe destacar que para BG5 sólo se definen tres TFC propios, los códigos de tipo TFC5, TFC6 y TFC8, donde sólo se usan las dos bandas 13 y 14.

En la Tabla 2.6 y Tabla 2.7 se muestran los códigos TFC para BG1 y BG5. En el estándar se denomina TFI (TFI, Time-Frequency Interleaved) a aquellos códigos que saltan en frecuencia entre las tres bandas de un BG, en este caso los códigos TFC1 al TFC4. FFI (FFI, Fixed-Frequency Interleaved) son aquellos códigos que solo usan una banda y no tienen salto en frecuencia, como TFC5 al TFC7, y por último TFI2 (TFI2, Two-band TFI) son aquellos códigos que realizan salto en frecuencia entre dos de las tres bandas de un BG, en este caso los códigos TFC8 al TFC10.

\section{TABLA 2.6. CANALIZACIÓN MB-OFDM SEGúN CóDIGOS TFC PARA BG1}

\begin{tabular}{c|c|c|c|c|c|c|c}
\hline \hline Código TFC & Secuencia básica / preámbulo & \multicolumn{6}{|c}{ Número de banda $\left(\mathbf{n}_{\mathbf{b}}\right)$ según TFC } \\
\hline 1 & 1 & 1 & 2 & 3 & 1 & 2 & 3 \\
\hline 2 & 2 & 1 & 3 & 2 & 1 & 3 & 2 \\
\hline 3 & 3 & 1 & 1 & 2 & 2 & 3 & 3 \\
\hline 4 & 4 & 1 & 1 & 3 & 3 & 2 & 2 \\
\hline 5 & 5 & 1 & 1 & 1 & 1 & 1 & 1 \\
\hline 6 & 6 & 2 & 2 & 2 & 2 & 2 & 2 \\
\hline 7 & 7 & 3 & 3 & 3 & 3 & 3 & 3 \\
\hline 8 & 8 & 1 & 2 & 1 & 2 & 1 & 2 \\
\hline 9 & 9 & 1 & 3 & 1 & 3 & 1 & 3 \\
\hline 10 & 10 & 2 & 3 & 2 & 3 & 2 & 3 \\
\hline \hline
\end{tabular}

TABLA 2.7. CANALIZACIÓN MB-OFDM SEgúN CóDIGOS TFC PARA BG5

\begin{tabular}{c|c|c|c|c|c|c|c}
\hline \hline Código TFC & Secuencia básica / preámbulo & \multicolumn{5}{|c}{ Número de banda $\left(\mathbf{n}_{\mathbf{b}}\right)$ según TFC } \\
\hline 5 & 5 & 13 & 13 & 13 & 13 & 13 & 13 \\
\hline 6 & 6 & 14 & 14 & 14 & 14 & 14 & 14 \\
\hline 8 & 8 & 13 & 14 & 13 & 14 & 13 & 14 \\
\hline \hline
\end{tabular}

En la Figura 2.14 se observa la canalización tiempo-frecuencia y su comportamiento de forma cualitativa para la transmision de paquetes de datos OFDM, en este caso con código TFC1 en BG1. También se muestra la definición cualitativa de parámetros temporales en la transmisión de paquetes de datos MB-OFDM UWB como son el prefijo cíclico, el periodo y el intervalo de guarda. 


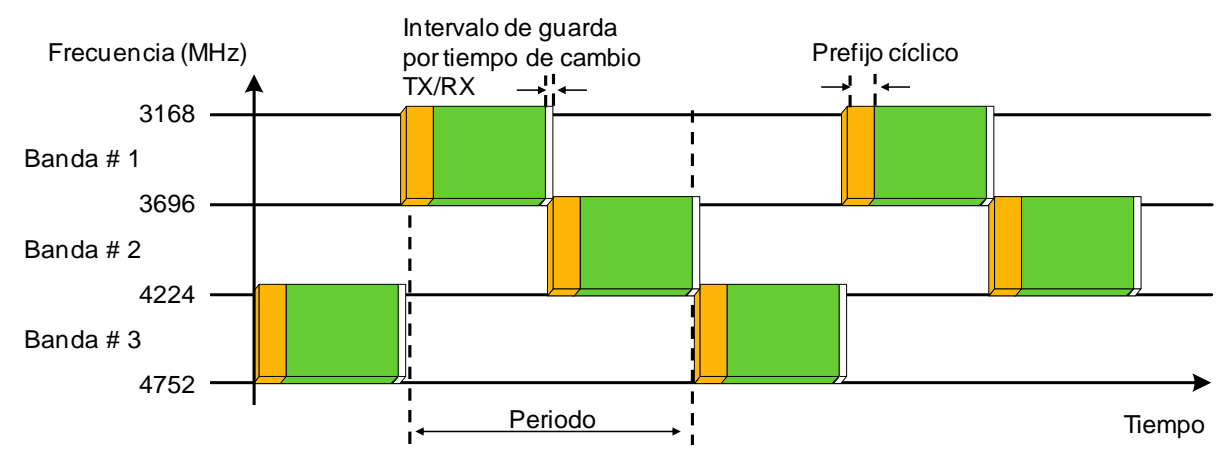

Figura 2.14. Transmisión tiempo-frecuencia de símbolos OFDM en MB-OFDM UWB con TFC1 en BG1.

Cada banda MB-OFDM UWB de ancho de banda de $528 \mathrm{MHz}$ está dividida en 128 subportadoras ortogonales entre sí y separadas $4.125 \mathrm{MHz}$. La separación entre portadoras establece la duración temporal del símbolo OFDM, de valor $242.42 \mathrm{~ns}$. Al símbolo OFDM se le añade un prefijo cíclico nulo de $70.08 \mathrm{~ns}$ para conformar una duración total de símbolo de 312.5 ns. Los parámetros temporales del símbolo OFDM se recogen en la Tabla 2.8 .

TABla 2.8. ParÁMETROS TEMPORALES de LA SEÑAl MB-OFDM báSICA

\begin{tabular}{l|l|l}
\hline \hline Parámetro & Descripción & Valor \\
\hline$f_{s}$ & Frecuencia de muestreo & $528 \mathrm{MHz}$ \\
\hline$N_{F F T}$ & Número total de subportadoras (FFT size $)$ & 128 \\
\hline$N_{D}$ & Número de subportadoras de datos & 100 \\
\hline$N_{P}$ & Número de subportadoras piloto & 12 \\
\hline$N_{G}$ & Número de subportadoras de guarda & 10 \\
\hline$N_{T}$ & Número total de subportadoras en uso & $122\left(=N_{D}+N_{P}+N_{G}\right)$ \\
\hline$D_{f}$ & Separación frecuencial entre subportadoras & $4.125 \mathrm{MHz}\left(=f s / N_{F F T}\right)$ \\
\hline$T_{F F T}$ & Periodo IFFT y FFT & $242.42 \mathrm{~ns}(\Delta \mathrm{f}-1)$ \\
\hline$N_{Z P S}$ & Número de muestras en zero-padded suffix & 37 \\
\hline$T_{Z P S}$ & Duración temporal de zero-padded suffix & $70.08 \mathrm{~ns}\left(=N_{Z P S} / f s\right)$ \\
\hline$T_{S Y M}$ & Intervalo entre símbolos & $312.5 \mathrm{~ns}\left(=T_{F F T}+T_{Z P S}\right)$ \\
\hline$F_{S Y M}$ & Tasa de símbolo & $3.2 \mathrm{MHz}\left(=T_{S Y M}-1\right)$ \\
\hline$N_{S Y M}$ & Número total de muestras por símbolo & $165\left(=N_{F F T}+N_{Z P S}\right)$ \\
\hline \hline
\end{tabular}

En el conjunto de 128 subportadoras de un símbolo OFDM existen 6 portadoras nulas, una de ellas la central, tres en la parte derecha o superior y dos en la parte izquierda o baja de la banda. El resto de subportadoras se dividen en 12 subportadoras piloto, 10 subportadoras de guarda y 100 portadoras de datos, como se puede observar en la Figura 2.15, donde la numeración de las subportadoras se presenta con los valores de -61 hasta +61 . 


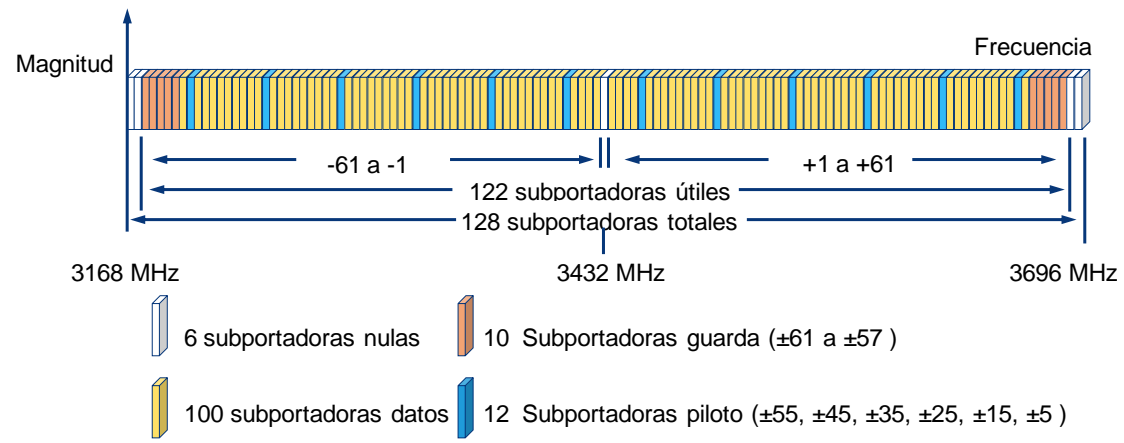

Figura 2.15. Configuración de subportadoras OFDM para MB-OFDM UWB.

El paquete MB-OFDM UWB está formado por una concatenación de símbolos OFDM con diversa funcionalidad, que va desde la sincronización, la estimación de canal, la cabecera del paquete, hasta la carga o payload de datos. El preámbulo de un paquete MB-OFDM está formado por aquellos símbolos dedicados a la sincronización y estimación de canal. Seguidamente se encuentra la cabecera del paquete, este subconjunto lo forman 42 símbolos OFDM como se muestra en la Tabla 2.9. La longitud del conjunto de datos del paquete UWB varía en función del régimen binario escogido. Para el máximo régimen binario MB-OFDM UWB de $480 \mathrm{Mbit} / \mathrm{s}$ la longitud máxima de datos en un paquete son 1968 símbolos OFDM.

TABLA 2.9. CARACTERÍSTICAS FUNCIONALES DE UN PAQUETE DE SEÑAL MB-OFDM UWB BÁSICA

\begin{tabular}{l|l|l}
\hline \hline Parámetro & Descripción & Valor \\
\hline$N_{p f}$ & $\begin{array}{l}\text { Número de símbolos de la secuencia de } \\
\text { sincronización }\end{array}$ & $\begin{array}{l}\text { Preámbulo estándar: } 24 \\
\text { Preámbulo burst: } 12\end{array}$ \\
\hline$T_{p f}$ & Duración de la secuencia de sincronización & $\begin{array}{l}\text { Preámbulo estándar: } 7.5 \mu \mathrm{s} \\
\text { Preámbulo burst: } 3.75 \mu \mathrm{s}\end{array}$ \\
\hline$N_{c e}$ & N. símbolos de la secuencia de estimación de canal & 6 \\
\hline$T_{c e}$ & Duración de la secuencia de estimación canal & $1.875 \mu \mathrm{s}$ \\
\hline$N_{\text {sync }}$ & Número de símbolos de preámbulo PLCP & $\begin{array}{l}\text { Preámbulo estándar: } 30 \\
\text { Preámbulo burst: } 18\end{array}$ \\
\hline$T_{\text {sync }}$ & Duración del preámbulo PLCP & $\begin{array}{l}\text { Preámbulo estándar: } \\
9.375 \mu \mathrm{s} \\
\text { Preámbulo burst: } 5.625 \mu \mathrm{s}\end{array}$ \\
\hline$N_{h d r}$ & Número de símbolos de la cabecera PLCP & 12 \\
\hline$T_{h d r}$ & Duración de la cabecera PLCP & $3.75 \mu \mathrm{s}$ \\
\hline$N_{\text {frame }}$ & Número de símbolos PSDU & $6 \times\left[\frac{8 \times L E N G T H+38}{N_{I B P 6 S}}\right]$ \\
\hline$T_{\text {frame }}$ & Duración de PSDU & $6 \times\left[\frac{8 \times L E N G T H+38}{N_{I B P G S}}\right] \times T_{S Y M}$ \\
\hline$N_{\text {packet }}$ & Número de símbolos total del paquete & $N_{s v n c}+N_{h d r}+N_{\text {frame }}$ \\
\hline$T_{p a c k e t}$ & Duración del paquete & $\left(N_{s y n c}+N_{h d r}+N_{f r a m e} \times T_{S Y M}\right.$ \\
\hline \hline
\end{tabular}


El estándar MB-OFDM UWB contempla dos tipos de modulaciones, QPSK y modulación de doble portadora (DCM, Dual Carrier Modulation), para transmitir los datos sobre las 100 subportadoras disponibles, y establece 10 regímenes binarios que van desde $53.3 \mathrm{Mbit} / \mathrm{s}$ hasta $480 \mathrm{Mbit} / \mathrm{s}$. La modulación QPSK se usa para transmisiones de hasta $200 \mathrm{Mbit} / \mathrm{s}$ con diferentes tasas de codificación (coding rates) según sea el régimen binario. Para los regímenes binarios de 320, 400 y $480 \mathrm{Mbit} / \mathrm{s}$ se utiliza la modulación DCM. En esta modulación la señal se representa por grupos de 4 bits, y cada grupo de 4 bits se mapea en 2 constelaciones diferentes, esta modulación es similar a la modulación 16 QAM. Cada constelación posee una subportadora que está separada 50 subportadoras de la anterior, es decir, alrededor de unos $206 \mathrm{MHz}$, lo que aumenta la robustez de la modulación ante desvanecimientos simultáneos de ambas subportadoras.

En la Tabla 2.10 se puede observar la correspondencia entre la modulación, las tasas de codificación $(R)$ y el régimen binario para comunicaciones MB-OFDM UWB.

TABla 2.10. PARÁMETros de MOdUlaCión dE LA SEÑAL MB-OFDM bÁSICA

\begin{tabular}{c|c|c|c|c|c|c}
\hline \hline $\begin{array}{c}\text { Régimen } \\
\text { Binario } \\
(\text { Mbit/s) }\end{array}$ & Modulación & $\begin{array}{c}\text { Tasa de } \\
\text { codificación } \\
(\mathbf{R})\end{array}$ & FDS & TDS & $\begin{array}{c}\text { Bits } \\
\text { codificados } \\
\text { por cada 6 } \\
\text { símb. OFDM } \\
\left(\mathbf{N}_{\text {CBP6S }}\right)\end{array}$ & $\begin{array}{c}\text { Bits de } \\
\text { información } \\
\text { por cada 6 } \\
\text { símb. OFDM } \\
\left(\mathbf{N}_{\text {IBP6S }}\right)\end{array}$ \\
\hline 53.3 & QPSK & $1 / 3$ & SI & SI & 300 & 100 \\
\hline 80 & QPSK & $1 / 2$ & SI & SI & 300 & 150 \\
\hline 106.7 & QPSK & $1 / 3$ & NO & SI & 600 & 200 \\
\hline 160 & QPSK & $1 / 2$ & NO & SI & 600 & 300 \\
\hline 320 & QPSK & $5 / 8$ & NO & SI & 600 & 375 \\
\hline 400 & DCM & $1 / 2$ & NO & NO & 1200 & 600 \\
\hline 480 & DCM & $3 / 4$ & NO & NO & 1200 & 900 \\
\hline \hline
\end{tabular}




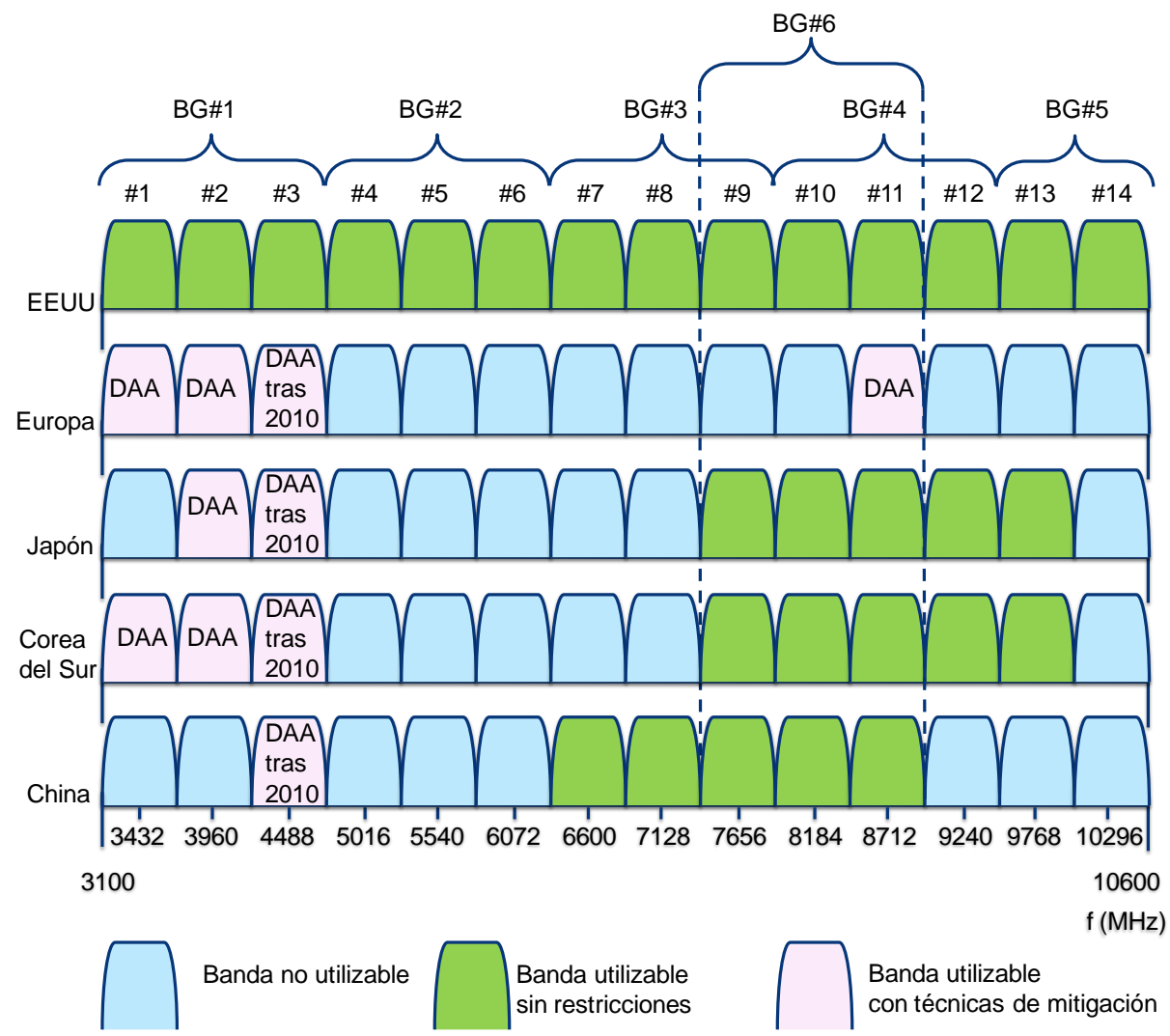

Figura 2.16. Estado actual de las regulaciones MB-OFDM UWB a nivel mundial, según WiMedia Alliance en Enero de 2009 [Wim].

En la Figura 2.16 se muestran las regulaciones internacionales que afectan actualmente a MB-OFDM UWB. En esta figura se observa como en muchos marcos regulatorios, como el europeo, no todas las bandas de frecuencias dadas por el estándar [ECMA368] están disponibles para su uso. A su vez, se observa cómo se introduce el concepto de técnica de mitigación DAA para la implementación adecuada de MB-OFDM UWB en ciertas bandas de frecuencia. Este tipo de técnicas tiene como objetivo detectar y evitar la interferencia con sistemas radio de banda estrecha con licencia en la banda de trabajo MB-OFDM UWB, mientras está en funcionamiento una transmisión UWB. La regulación espectral de UWB y la implementación de técnicas de mitigación de la interferencia son cuestiones reflejadas en la normativa de los organismos reguladores internacionales referente al despliegue futuro de MB-OFDM UWB [ECC06.04]. En la Figura 2.16 se indican las bandas de frecuencia donde se deben implementar técnicas DAA para asegurar el correcto despliegue 
de MB-OFDM UWB. A su vez, también se incluye la moratoria para la implantación de técnicas DAA en Europa y Asia hasta finales de 2010.

Tras revisar las tecnologías radio WiMAX y MB-OFDM UWB se exponen los principios de coexistencia de estas tecnologías en escenarios WPAN.

\subsection{Principios de coexistencia en WPAN}

Las redes personales inalámbricas (WPAN) se caracterizan por proveer servicios en zonas de cobertura radio menores a 10 metros. En este tipo de entornos, la presencia de los sistemas de comunicaciones de elevado régimen binario como UWB y WiMAX provoca situaciones de interferencia con otros sistemas de banda estrecha, como son Wi-Fi o Bluetooth.

Por otra parte, los sistemas MB-OFDM UWB y Mobile WiMAX se implementan en el espacio radioeléctrico por debajo de $10.6 \mathrm{GHz}$, lo cual provoca situaciones de interferencia entre ambos. Esta interferencia se produce fundamentalmente debido a que el modo UWB mandatory se implementa en la banda de frecuencias de $3.1 \mathrm{GHz}$ a 4.8 GHz y Mobile WiMAX se implementa en la banda de frecuencias entre $3.4 \mathrm{GHz}$ a $3.8 \mathrm{GHz}$ [IEEE80216e].

La tecnología Mobile WiMAX se está desplegando en las redes inalámbricas actuales gracias a los productos comerciales de compañías como Alvarion, Aperto o Motorola. Por ejemplo, la solución llave en mano Mobile WiMAX de Alvarion [ALV09c] cumple con el estándar IEEE 802.16e y permite extender el acceso WiMAX fijo [IEEE80216d] a entornos de movilidad. Según sus especificaciones, dispone de interfaces compatibles con los estándares de comunicaciones móviles 3GPP con compatibilidad de contenidos multimedia IP (IMS, IP Multimedia Subsystem) y de cable. A su vez, también existen ordenadores portátiles preparados para comunicaciones inalámbricas basadas en WiMAX [LEN09], módems USB para conexiones Mobile WiMAX [FRA09] y circuitos integrados como la plataforma Centrino $2^{\text {tm }}$ de INTEL Inc. con soporte para comunicaciones basadas en IEEE 802.16 [INT09]. Esta disponibilidad de productos confirma la presencia de WiMAX en diferentes escenarios, desde WPAN hasta WWAN.

La tecnología MB-OFDM UWB se está desarrollando de forma importante en entornos WPAN gracias a su inclusión en productos comerciales. El informe público realizado en el seno del proyecto europeo UCELLS sobre la evolución comercial de la tecnología UWB [Llo09], muestra aplicaciones MB-OFDM UWB como son el USB inalámbrico (WUSB) [USB09], la transmisión de datos multimedia de alta definición HDMI (HDMI, High-Definition Multimedia Interface) de forma inalámbrica o Wireless $H D M I$, la inclusión en dispositivos portátiles o la elección de MB-OFDM UWB como 
base tecnológica para Bluetooth 3.0 [BLU09]. Este informe indica la creciente evolución de UWB y cómo su presencia en entornos WPAN es cada vez mayor gracias a los futuros formatos WUSB y Bluetooth 3.0 [USB09], [BLU09].

En la Figura 2.17 se presentan dispositivos comerciales con tecnología Mobile WiMAX y MB-OFDM UWB.

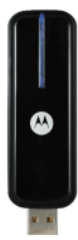

(a)

(b)

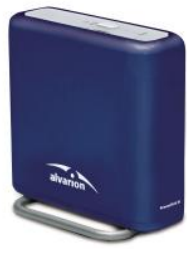

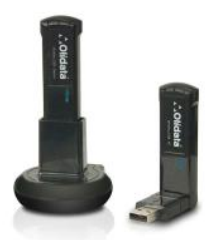

(c)

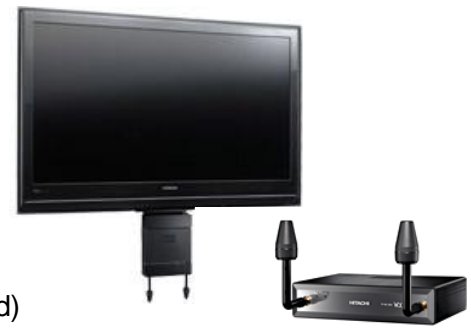

Figura 2.17. Dispositivos Mobile WiMAX (a) módem USB Motorola wi4 WiMAX USBw 100 [MOT09], (b) dispositivo Mobile WiMAX BreezeMAX Si de Alvarion Ltd. [ALV09b], y de dispositivos MB-OFDM UWB, (c) dispositivos Wireless USB Adapter Set de Olidata S.p.A. [OLI09], (d) televisión WOOO LCD HITACHI con Wireless HDMI [HIT09].

Este tipo de productos se enmarcan dentro de las comunicaciones radio en entornos WPAN. Los productos WiMAX permiten el aumento de la cobertura radio para servicios móviles de elevado régimen binario en entornos interiores. A su vez, los productos UWB proporcionan conexiones de muy elevado régimen binario de corto alcance. Comparando los regímenes binarios de ambas tecnologías, hasta $480 \mathrm{Mbit} / \mathrm{s}$ en MB-OFDM UWB y menores de $100 \mathrm{Mbit} / \mathrm{s}$ en WiMAX, y comparando también el objetivo de sus aplicaciones, se puede concluir que ambas son tecnologías complementarias y coexistentes en entornos WPAN.

El estudio de la coexistencia y complementariedad entre tecnologías UWB y WiMAX es esencial para definir de forma precisa el marco regulador para la implementación adecuada de ambas tecnologías. Este estudio es un paso previo para alcanzar los objetivos de esta Tesis Doctoral.

La coexistencia espectral en la tecnología UWB se especifica en el estándar ECMA-368 [ECMA368] y en las regulaciones internacionales mediante técnicas de mitigación de la interferencia y procedimientos que aseguren una correcta implementación de UWB en presencia de otras tecnologías radio. Es decir, UWB debe permitir la interoperabilidad con otros sistemas de radiocomunicaciones desplegados en el mismo escenario. Por ejemplo, existen redes WPAN desplegadas como Wi-Fi, ZigBee y Bluetooth. A su vez, en entornos WPAN están presentes otras redes como GSM, HSPA y WiMAX 802.16d/e. Este tipo de redes WPAN y sistemas de 
comunicaciones inalámbricas conforman las situaciones de interferencia y coexistencia que UWB debe resolver para su adecuado despliegue.

A este respecto, la regulación UWB en el ámbito de la Unión Europea propone técnicas para la mitigación de la interferencia de UWB en sistemas radio con licencia. La decisión del 21 de abril de 2009 de la comisión europea, para la utilización armonizada del espectro radioeléctrico para los equipos que utilizan tecnología de banda ultra-ancha [DEC09], propone, basándose en estudios de la CEPT [ECC08.10], la implementación de técnicas de mitigación de la interferencia, en particular DAA, para sistemas MB-OFDM UWB, y LDC para implementaciones UWB de tipo impulsivo.

En las normativas UWB de Japón y Corea del Sur también se establecen técnicas de mitigación de la interferencia para el correcto funcionamiento de UWB ante la presencia de otros sistemas radio con licencia en su espectro de emisión, principalmente mediante técnicas DAA [Nik08].

\subsubsection{Técnica de mitigación por reducción del ciclo de trabajo}

La técnica de mitigación LDC propuesta en la regulación [ECC08.10] tiene como objetivo regular la emisión y mitigar las interferencias de los dispositivos UWB dedicados a posicionamiento y a aplicaciones de baja régimen binario. La tecnología radio UWB implementada en este tipo de aplicaciones es la de tipo impulsiva, tal y como se muestra en el grupo de trabajo IEEE 802.15 TG4a.

Este tipo de tecnología UWB tiene como objetivo redes de sensores. Por ejemplo, en el ámbito doméstico, aplicaciones como la domótica o de ayuda al geo-posicionamiento detallado para vigilancia de personas. Otras aplicaciones se sitúan en entornos industriales, como son la localización de procesos en una cadena productiva, de elementos dentro de un almacén, la gestión del personal o aplicaciones de control y seguimiento de flotas. Todas estas aplicaciones poseen un bajo factor de actividad, es decir, no necesitan realizar transmisiones continuas de datos.

Los principales parámetros para las transmisiones de ráfagas (burst) que contienen pulsos de tipo UWB que implementan LDC son:

- Ciclo de trabajo, porcentaje sobre el tiempo total de transmisión en el cual se transmiten señales burst válidas.

- $T_{o n}$, duración de un pulso de tipo burst, sin tener en cuenta el número de pulsos UWB que contenga el mismo. 
- $\quad T_{o f f}$, intervalo de tiempo entre dos burst mientras la transmisión UWB se mantiene en estado de inactividad (IDLE).

En la decisión europea para el uso de UWB basado en técnicas de mitigación [ECC08.10] encontramos los siguientes parámetros referidos a la implementación de UWB con LDC, resumidos en la Tabla 2.11.

\section{TABLA 2.11. PARÁMETROS PARA LA IMPLEMENTACIÓN DE LA TÉCNICA DE MITIGACIÓN LDC PARA UWB}

\begin{tabular}{l|l}
\hline \hline$T_{\text {on }}$ máximo & $5 \mathrm{~ms}$ \\
\hline $\mathrm{T}_{\text {off }}$ medio & $\geq 38 \mathrm{~ms}$ (promediado sobre 1 segundo) \\
\hline$\sum T_{\text {off }}$ & $>950 \mathrm{~ms}$ por segundo \\
\hline$\sum T_{\text {on }}$ & $<18 \mathrm{~s}$ por hora \\
\hline EIRP & $\begin{array}{l}\text { La densidad espectral media EIRP y la } \\
\text { potencia de pico EIRP se definen de acuerdo a } \\
\text { la normativa vigente durante el intervalo } T_{\text {on }} .\end{array}$ \\
\hline \hline
\end{tabular}

\subsubsection{Técnica de mitigación por detección y exclusión}

Las técnicas de mitigación basadas en DAA para MB-OFDM UWB se definen para controlar y prevenir las interferencias sobre aplicaciones BWA, como son los sistemas WiMAX en la banda 3.4-4.2 GHz y los servicios de radiolocalización en las bandas 3.1-3.4 GHz y 8.5-9 GHz.

La propuesta realizada en el informe de Junio de 2008 del ECC [ECC120] detalla el protocolo DAA y su funcionalidad a partir de la definición de diferentes zonas, donde se autoriza un determinado nivel de potencia emitida UWB. Estas zonas corresponden con el nivel de aislamiento mínimo necesario entre el sistema radio víctima potencial y el sistema interferente UWB. Para los diferentes sistemas radio víctimas, se definen en cada zona unos niveles de potencia máxima o niveles de protección para la transmisión UWB. Estos niveles de protección, junto con el aislamiento mínimo establecido, tienen como finalidad no interferir en la funcionalidad operativa de los sistemas radio víctimas basándose en la definición de protección equivalente definida en [ECC06.04]. Las zonas de protección definidas para la técnica de mitigación DAA son las zonas de protección (1, 2, 3), localizadas en la banda de $3.4 \mathrm{GHz}$ a $4.2 \mathrm{GHz}$, y dos zonas de protección (A, B) localizadas en la banda de $3.1 \mathrm{GHz}$ a $3.4 \mathrm{GHz}$ y 8.5 GHz a $9 \mathrm{GHz}$.

Los dispositivos UWB que implementan DAA, antes de establecer un enlace UWB de comunicaciones, deben realizar una monitorización de las señales RF en determinadas bandas de frecuencia presentes en su entorno de trabajo durante un tiempo 
mínimo, para poder detectar cualquier señal radio en funcionamiento que se considere víctima. La duración de esta fase inicial se denomina tiempo inicial de testeo de disponibilidad del canal. Según los datos obtenidos durante el análisis del canal de transmisión, el dispositivo UWB determinará en qué zona de trabajo DAA se encuentra y cómo debe configurarse al respecto. Además, los dispositivos UWB con DAA deben ser capaces de detectar cambios en las señales RF de su entorno para adaptarse y cambiar su configuración de emisión máxima.

En la normativa europea se definen los procedimientos de selección de la zona de aislamiento y los niveles máximos de potencia emitida UWB adecuados.

(i) Para la banda de 3.1-3.4 GHz y $8.5-9 \mathrm{GHz}$ se establece un solo umbral de detección $D_{\text {thresh }}$ que define dos zonas de protección, zona A y zona B. Ante señales detectadas con niveles por encima del umbral, se limita la potencia de emisión UWB a niveles inferiores a $-41.3 \mathrm{dBm} / \mathrm{MHz}$ para toda la banda bajo estudio, es decir, $300 \mathrm{MHz}$ para la banda de $3.1 \mathrm{GHz}$ a $3.4 \mathrm{GHz}$ y $500 \mathrm{MHz}$ para la banda de $8.5 \mathrm{GHz}$ a $9 \mathrm{GHz}$, situándose entonces en la zona $\mathrm{A}$. Si el nivel detectado es inferior al umbral, el interferente UWB puede emitir con niveles de $-41.3 \mathrm{dBm} / \mathrm{MHz}$, y se sitúa en la zona $\mathrm{B}$.

(ii) Para la banda de $3.4-4.2 \mathrm{GHz}$ se propone un sistema DAA basado en dos umbrales de señal detectada en el canal de transmisión o enlace de subida de $-38 \mathrm{dBm}$ y de $-61 \mathrm{dBm}$. Así, se establecen niveles de emisión UWB limitados según el umbral de señal detectada por el dispositivo UWB. Si el nivel de señal está por debajo de $-38 \mathrm{dBm}$, se limita la señal UWB a $-80 \mathrm{dBm} / \mathrm{MHz}$ y el dispositivo UWB se encuentra en la zona 1. Si el nivel de la señal detectada se sitúa entre $-38 \mathrm{dBm}$ y $-61 \mathrm{dBm}$, se limita la señal UWB a $-65 \mathrm{dBm} / \mathrm{MHz}$ y el dispositivo UWB se encuentra en la zona 2. Finalmente, si el nivel de las señales detectadas es igual o menor a $-61 \mathrm{dBm}$, se establece el límite de emisiones UWB en $-41.3 \mathrm{dBm} / \mathrm{MHz}$ y se sitúa al dispositivo UWB en la zona 3.

Para señales del enlace de subida detectadas en las bandas de $3.4-3.6 \mathrm{GHz}$ o 3.6-3.8 GHz, la banda de exclusión es de $200 \mathrm{MHz}$. Además, el desarrollo de la técnica DAA para la detección de servicios BWA permite reducir la banda de exclusión a un mínimo de $20 \mathrm{MHz}$, debido a las características de los sistemas BWA.

La combinación de este umbral de señal detectada, junto con el tiempo de testeo de la disponibilidad del canal y la probabilidad de detección, permiten asegurar según el informe [ECC120] la protección de los terminales BWA en más del $99.75 \%$ del tiempo de funcionamiento.

El funcionamiento de DAA no sólo es obligatorio para los dispositivos UWB de forma individual, sino que también es necesario que lo implementen y lo pongan en 
funcionamiento aquellos que conforman redes UWB. Así, durante el funcionamiento de una red UWB, formada por al menos dos dispositivos, los dispositivos deben tener la posibilidad de negociar quién de ellos realiza la detección y compartir posteriormente la información relativa a este proceso de detección de DAA. Por ejemplo, en la transmisión de un archivo de gran tamaño entre dos dispositivos UWB, el receptor puede realizar las tareas de detección DAA y luego compartir la información con el transmisor. Además, es conveniente que quien realice estas tareas de detección sea un dispositivo alimentado continuamente para extender la duración de la batería en dispositivos UWB móviles. Ante estos aspectos de funcionamiento se recomienda que la detección continua sea el modo empleado en todo dispositivo UWB cuando éste se encuentre en operaciones de recepción de datos.

Los principales parámetros DAA para comunicaciones MB-OFDM UWB son:

- Tiempo mínimo inicial de testeo de la disponibilidad del canal, $T_{\text {avail }}$ : tiempo mínimo durante el cual el dispositivo UWB busca posibles señales radio víctimas antes de comenzar su funcionamiento o Power On.

- Umbral de señal detectada, $D_{\text {thresh }}$ : se define como la potencia recibida por parte de la posible víctima radio. Define la transición entre dos zonas de protección adyacentes. Este umbral de detección se especifica para el conector de antena asumiendo una ganancia de antena de $0 \mathrm{dBi}$ en el proceso de detección. También se puede expresar como un límite de campo eléctrico.

- Nivel de exclusión (avoidance) o densidad de potencia de transmisión UWB máxima: máxima densidad de potencia de transmisión que se configura en el dispositivo UWB dependiendo de la zona de protección en la cual se encuentra.

- Ancho de banda de exclusión por defecto: indica el ancho de banda mínimo de la señal víctima que requiere protección.

- Tiempo máximo de DAA, $T_{\text {avoid }}$ : máxima duración temporal entre un cambio en las condiciones RF ambientales en el entorno de un dispositivo UWB y la adaptación necesaria de los parámetros de funcionamiento del mismo.

- Probabilidad de detección: probabilidad de acertar en la decisión correcta en un dispositivo MB-OFDM UWB que implemente DAA debido a la presencia de una señal víctima antes de comenzar la transmisión o debido a cambios en la configuración RF durante el funcionamiento de un dispositivo UWB. 
Los parámetros técnicos de la Tabla 2.12 y Tabla 2.13 se han obtenido gracias a estudios realizados sobre escenarios de sistemas de radiolocalización en presencia de dispositivos MB-OFDM UWB [ECC120], [ECC08].

TABla 2.12. PARÁMEtros DAA PARA LA BANDA 3.1 - 3.4 GHz

\begin{tabular}{|c|c|c|c|}
\hline Parámetro & & Zona A & Zona B \\
\hline $\begin{array}{l}\text { Tiempo mínimo inicial de } \\
\text { disponibilidad del canal }\end{array}$ & $\bar{T} T_{\text {avail }}$ & \multicolumn{2}{|c|}{$14 \mathrm{~s}$} \\
\hline Tiempo máximo de DAA & $T_{\text {avoid }}$ & \multicolumn{2}{|c|}{$150 \mathrm{~s}$} \\
\hline $\begin{array}{l}\text { Probabilidad de detección en el modo "escucha } \\
\text { antes de habla" para el patrón de test dado por ETSI } \\
\text { EN } 302065\end{array}$ & & \multicolumn{2}{|c|}{$99 \%$} \\
\hline $\begin{array}{l}\text { Probabilidad de detección operando en modo de } \\
\text { detección continua cuando el UWB está } \\
\text { funcionando según el patrón de test dado por ETSI } \\
\text { EN302065 }\end{array}$ & & \multicolumn{2}{|c|}{$97 \%$} \\
\hline Umbral de detección & $D_{\text {thresh }}$ & $-38 \mathrm{dBm}$ & $-38 \mathrm{dBm}$ \\
\hline $\begin{array}{l}\text { Nivel de exclusión o eludible (Densidad espectral } \\
\text { de potencia transmisión UWB máxima) }\end{array}$ & & $-70 \mathrm{dBm} / \mathrm{MHz}$ & $-41.3 \mathrm{dBm} / \mathrm{MHz}$ \\
\hline Ancho de de banda eludible por defecto & & \multicolumn{2}{|c|}{$300 \mathrm{MHz}$} \\
\hline
\end{tabular}

TABLA 2.13. PARÁMETros DAA PARA LA BANDA 8.5 - 9 GHz

\begin{tabular}{|c|c|c|c|}
\hline Parámetro & & Zona A & Zona B \\
\hline $\begin{array}{l}\text { Tiempo mínimo inicial } \\
\text { disponibilidad del canal }\end{array}$ & $\overline{T_{\text {avail }}}$ & \multicolumn{2}{|c|}{$14 \mathrm{~s}$} \\
\hline Tiempo máximo de DAA & & \multicolumn{2}{|c|}{$150 \mathrm{~s}$} \\
\hline $\begin{array}{l}\text { Probabilidad de detección en el modo "escucha } \\
\text { antes de habla" para el patrón de test dado por ETSI } \\
\text { EN } 302065\end{array}$ & & \multicolumn{2}{|c|}{$99 \%$} \\
\hline $\begin{array}{l}\text { Probabilidad de detección operando en modo de } \\
\text { detección continua cuando el UWB está } \\
\text { funcionando según el patrón de test dado por ETSI } \\
\text { EN302065 }\end{array}$ & & \multicolumn{2}{|c|}{$97 \%$} \\
\hline Umbral de detección & $D_{\text {thresh }}$ & $-61 \mathrm{dBm}$ & $-61 \mathrm{dBm}$ \\
\hline $\begin{array}{l}\text { Nivel de exclusión o eludible (Densidad espectral } \\
\text { de potencia transmisión UWB máxima) }\end{array}$ & & $-65 \mathrm{dBm} / \mathrm{MHz}$ & $-41.3 \mathrm{dBm} / \mathrm{MHz}$ \\
\hline Ancho de banda eludible por defecto & & \multicolumn{2}{|c|}{$500 \mathrm{MHz}$} \\
\hline
\end{tabular}

En la Tabla 2.14 y Tabla 2.15 se presentan los parámetros DAA para la banda 3.4-4.2 GHz. Aparte de estos parámetros técnicos recomendados por la ECC, también se observan otros datos como el tiempo de DAA adecuado según el tipo de servicio que se esté implementando por los terminales BWA. 
TABla 2.14. PARÁMetros DAA PARa la BANDA 3.4 - 4.2 GHz

\begin{tabular}{|c|c|c|c|c|}
\hline Parámetro & & Zona 1 & Zona 2 & Zona 3 \\
\hline $\begin{array}{l}\text { Tiempo mínimo inicial de } \\
\text { testeo de la disponibilidad del } \\
\text { canal }\end{array}$ & $T_{\text {avail }}$ & \multicolumn{3}{|c|}{$5.1 \mathrm{~s}$} \\
\hline $\begin{array}{l}\text { Probabilidad de detección para } \\
\text { la operación inicial de } \\
\text { detección después } \\
\text { encenderse el dispositivo UWB. }\end{array}$ & & \multicolumn{3}{|c|}{$99 \%$} \\
\hline $\begin{array}{l}\text { Umbral de detección para el } \\
\text { enlace ascendente }\end{array}$ & $\begin{array}{l}D_{\text {thresh }} \\
\text { (UL) }\end{array}$ & $\begin{array}{l}\geq-38 \mathrm{dBm} \\
\left(D_{\text {thresh } 1)}\right)\end{array}$ & $\begin{array}{l}<-38 \mathrm{dBm} \\
\left(D_{\text {thresh } 1}\right) \\
\geq-61 \mathrm{dBm} \\
\left(D_{\text {thresh } 2)}\right. \\
\end{array}$ & $\begin{array}{l}<-61 \mathrm{dBm} \\
\left(D_{\text {thresh } 2)}\right)\end{array}$ \\
\hline $\begin{array}{l}\text { Nivel de exclusión o eludible } \\
\text { (Densidad espectral de potencia } \\
\text { transmisión UWB máxima) }\end{array}$ & & $-80 \mathrm{dBm} / \mathrm{MHz}$ & $-65 \mathrm{dBm} / \mathrm{MHz}$ & $-41.3 \mathrm{dBm} / \mathrm{MHz}$ \\
\hline $\begin{array}{l}\text { Ancho de de banda eludible por } \\
\text { defecto }\end{array}$ & & \multicolumn{3}{|c|}{$3.4-3.6 \mathrm{GHz}$ o $3.6-3.8 \mathrm{GHz}$} \\
\hline
\end{tabular}

\section{TABLA 2.15. TIEMPO DAA SEGÚN SERVICIO BWA PARA 95\% PROBABILIDAD DE DETECCIÓN}

\begin{tabular}{c|c|c}
\hline \hline $\begin{array}{c}\text { Modo para test del } \\
\text { Sistema BWA }\end{array}$ & Tiempo de DAA & $\begin{array}{c}\text { Probabilidad de detección (para } \\
\text { un modo de funcionamiento de } \\
\text { detección continua)) }\end{array}$ \\
\hline VoIP & $2 \mathrm{~s}$ & $95 \%$ \\
\hline Navegación Web & $15 \mathrm{~s}$ & $95 \%$ \\
\hline Modo Sleep o espera & $60 \mathrm{~s}$ & $95 \%$ \\
\hline Difusión Multimedia & $15 \mathrm{~s}$ & $95 \%$ \\
\hline \hline
\end{tabular}

Dentro de la literatura referente a la implementación de técnicas DAA en terminales UWB, cabe destacar los trabajos de [Mis07a] y [Giu08]. Por ejemplo, en [Mis07a] se exponen diferentes métodos DAA como la anulación de subportadoras donde se detecta la presencia de la víctima radio, el enventanado en el dominio temporal para aumentar la atenuación en la banda deseada, la cancelación de subportadoras en la banda de interferencia y el uso de técnicas de codificación de canal para reubicar esa información UWB en subportadoras adyacentes. También se propone el uso de un filtrado tipo notch centrado en las subportadoras UWB que deben ser evitadas por la interferencia introducida por sistemas radio con licencia. Todas estas propuestas de implementación práctica de DAA requieren atenuaciones del orden de $-35 \mathrm{~dB}$ a $-40 \mathrm{~dB}$ para cumplir con los parámetros técnicos DAA, lo cual supone una reprogramación de los circuitos internos de los terminales UWB que puede llegar a ser muy costosa computacionalmente. 
Para una correcta implementación de las técnicas avanzadas de mitigación de la interferencia aquí expuestas, es necesario ampliar el estudio a otros escenarios de coexistencia. Este estudio es necesario para los trabajos expuestos en los siguientes capítulos de esta Tesis Doctoral. 



\section{Capítulo 3. Coexistencia radio en entornos de redes personales}

Tal y como se ha comentado en el capítulo 2 de esta Tesis Doctoral, la demanda de servicios inalámbricos de elevado régimen binario proporcionando conectividad permanente y ubicua puede ser satisfecha mediante las tecnologías inalámbricas WiMAX y UWB. A su vez, la creciente penetración de las tecnologías inalámbricas en el mercado residencial, requiere un estudio de la coexistencia entre estos sistemas inalámbricos para asegurar su adecuada operación, una vez desplegados de forma masiva. La coexistencia entre ambos sistemas se ha establecido mediante la regulación en vigor que indica las bandas de frecuencia y las densidades espectrales potencia máxima de operación. Sin embargo, el futuro despliegue masivo de estos sistemas en escenarios comunes de aplicación y la evolución de las técnicas de transmisión en radiofrecuencia son motivos por los cuales es necesario investigar en detalle las coexistencia de estos dos estándares inalámbricos. En concreto:

(i) La regulación vigente se ha basado en estudios realizados en escenarios y ejemplos de aplicación convencionales. Sin embargo, las técnicas de integración de la transmisión óptica y radio en las redes de acceso integradas tienen como objetivo la provisión simultánea de múltiples señales inalámbricas, por ejemplo, mediante la implementación de clústeres de pico-celdas como se propone en el proyecto UCELLS [UCELLS]. Esto supone considerar la evaluación de nuevos escenarios de coexistencia entre UWB y WiMAX para adecuar la regulación actual y proponer cambios si fuese necesario.

(ii) La evolución de las técnicas de transmisión y recepción de radiofrecuencia, en las cuales se añade cierta inteligencia mediante la introducción de técnicas avanzadas de mitigación de la interferencia, como detección y exclusión DAA, y la utilización 
de técnicas que tienen en cuenta el entorno radio de los transmisores y receptores, conocidas como radios cognitivas (cognitive radio) [Mit99].

En este capítulo se expone el estudio de la coexistencia radio en escenarios de redes personales entre las tecnologías inalámbricas UWB y WiMAX. El estudio se ha centrado en la tecnología UWB basada en MB-OFDM UWB [ECMA368], puesto que es la implementación de la tecnología UWB con mayor disponibilidad en el mercado de la electrónica de consumo. Mientras que para el caso de las comunicaciones WiMAX se ha centrado en el estándar basado en IEEE 802.16e [IEEE80216e]. En primer lugar, se identifican los parámetros clave de la coexistencia en escenarios de redes personales o WPAN para ambas tecnologías. Posteriormente, en base a las medidas experimentales realizadas, se proponen márgenes de protección para la coexistencia e interoperabilidad de ambas en entornos WPAN. Estos márgenes de protección muestran la necesidad de implementar técnicas de mitigación de la interferencia para asegurar la coexistencia de ambas tecnologías y su futuro despliegue.

\subsection{Coexistencia entre MB-OFDM UWB y WiMAX 802.16e}

El estudio experimental se ha centrado en las tecnologías MB-OFDM UWB y WiMAX 802.16e debido a su complementariedad y la alta probabilidad de coexistencia a corto plazo. Esta complementariedad radica en que ambas tecnologías proporcionan elevados regímenes binarios y en su coexistencia en escenarios comunes, como edificios de oficinas. UWB es capaz de proporcionar regímenes binarios de hasta $1 \mathrm{Gbit} / \mathrm{s}$ [Lun06] y WiMAX de hasta $100 \mathrm{Mbit} / \mathrm{s}$ según los desarrollos IEEE 802.16m. A su vez, UWB se define para enlaces de corta distancia, mientras que WiMAX permite la extensión de la cobertura inalámbrica en entornos de difícil acceso como interiores de edificios. Además, la canalización espectral de UWB y WiMAX por debajo de 10.6 GHz indica una clara interferencia entre la tecnología WiMAX 802.16e centrada en $3.5 \mathrm{GHz}$ y la primera banda MB-OFDM UWB BG1 mandatory implementada entre $3.168 \mathrm{GHz}$ y $4.758 \mathrm{GHz}$.

El escenario WPAN de referencia utilizado para el estudio de la coexistencia de UWB y WiMAX contempla aplicaciones como ordenadores equipados con ambas tecnologías [LEN09], dispositivos UWB como llaves USB inalámbricas [OLI09] y dispositivos móviles WiMAX [MOT09], como se observa en la Figura 3.1. 


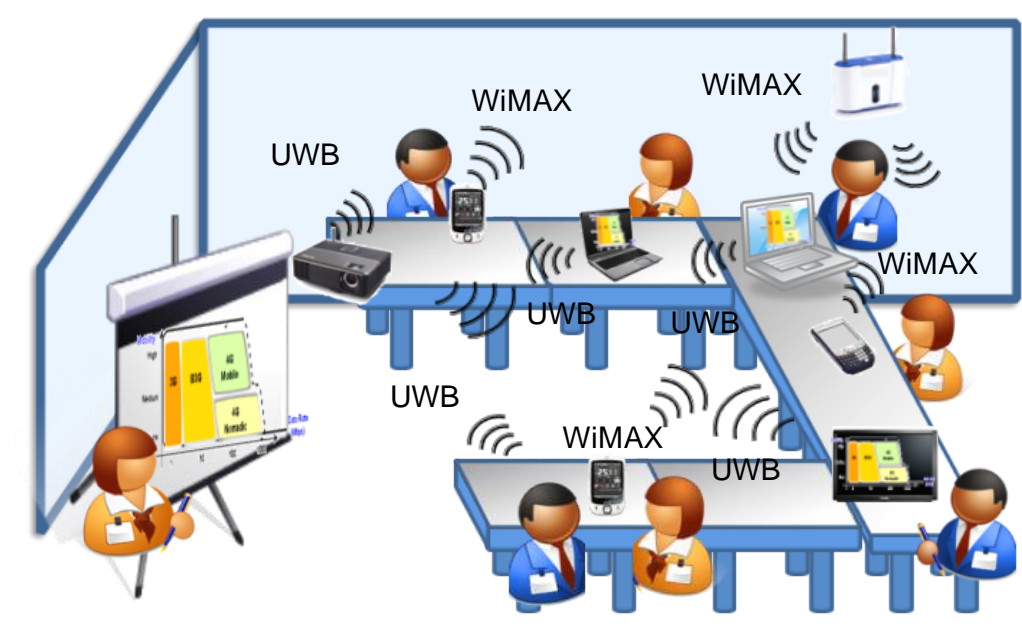

Figura 3.1. Escenario conceptual de coexistencia radio entre UWB y WiMAX.

La futura interoperabilidad entre UWB y otras tecnologías radio presentes en su entorno de aplicación ha sido regulada mediante el uso de técnicas de mitigación de la interferencia como DAA [ECC120], como se comentó en el capítulo 2 de esta Tesis Doctoral. Este tipo de técnicas de mitigación permite definir las capacidades de detección y exclusión controlada de la interferencia UWB sobre posibles víctimas radio como las comunicaciones WiMAX o servicios de radiolocalización. Este tipo de cuestiones se alinean en el marco de trabajo de las técnicas de conocimiento de información espectral de las transmisiones radio dentro del conjunto de futuras aplicaciones conocidas como cognitive radio [Mis07b].

El estudio de la interferencia entre UWB y otras tecnologías de banda estrecha como UMTS, GSM, GPS o la televisión digital por satélite, ha sido investigado tanto analíticamente como experimentalmente en [Por03], [Giu05], [Cot06], [Cas07]. Sin embargo, el impacto de UWB sobre las tecnologías WiMAX en la banda de $3.1 \mathrm{GHz}$ a 4.2 GHz solo se ha investigado de forma analítica en [Rah07], [Sno07]. También cabe destacar que se han publicado estudios analíticos sobre los procedimientos DAA para la coexistencia de UWB y WiMAX, analizando parámetros importantes como la probabilidad de detección [Mis07a], [Rah08], [Fac09].

En esta Tesis Doctoral se ha evaluado la coexistencia de MB-OFDM UWB y WiMAX 802.16e en un escenario WPAN de tipo oficina. Este escenario es una sala de reuniones perteneciente a la Universidad Politécnica de Valencia, que se caracteriza por el uso de materiales comunes como plásticos, cementos y maderas tanto en su mobiliario como en su concepción estructural. Para realizar el estudio experimental en 
primer lugar, es necesario definir las características de esta sala y como estas delimitan el modelo del canal de transmisión sobre el que se va a trabajar.

Los grupos TG3a y TG4a en el seno de IEEE 802.15 trabajan en la estandarización de un modelo de canal común para la evaluación de las comunicaciones UWB. El modelo de canal UWB en IEEE 802.15.3 se estableció en 2003 [Foe03], y actualmente, en el grupo TG4a, se está desarrollando un modelo de canal UWB para escenarios de baja velocidad. En el estudio experimental realizado en esta Tesis Doctoral se ha adoptado el modelo de canal establecido por el grupo TG3a para escenarios de corta distancia y transmisiones UWB de elevados regímenes binarios. A continuación se exponen las principales características de este modelo estándar del canal UWB.

\subsubsection{Modelo estándar del canal UWB}

El modelo estándar del canal UWB presentado en IEEE 802.15 TG3a por [Foe03], se basa en el conocido modelo de canal de Saleh-Valenzuela (SV) [Sal87a], pero variando la estadística de desvanecimiento del canal para adaptarla a las condiciones radioeléctricas que suponen las comunicaciones UWB. Para una mayor profundidad en los aspectos matemáticos que rodean a este modelo de canal de propagación UWB, es conveniente revisar el informe [Foe03]. Los parámetros característicos más relevantes de este modelo, así como los diferentes modelos de propagación a pequeña escala son los siguientes:

- Pérdidas de propagación o path loss. En el estándar se especifican estas pérdidas de propagación en espacio libre, con una frecuencia central $f_{c}=\sqrt{f_{L} f_{H}}$, donde $f_{L}$ y $f_{H}$ son los límites de frecuencia superior e inferior del espectro de la señal a $-10 \mathrm{~dB}$.

- Desvanecimiento lento o shadowing. Se asume que el shadowing del canal UWB sigue una distribución lognormal con una desviación estándar de $3 \mathrm{~dB}$ sobre la potencia media recibida, es decir, siendo el shadowing $X_{\sigma}[d B] \approx N\left(0, \sigma^{2}\right)$, donde $N\left(0, \sigma^{2}\right)$ es una distribución Gaussiana de media nula y varianza $\sigma^{2}$, con $\sigma=3 \mathrm{~dB}$.

- Desvanecimientos a pequeña escala o small-scale fading. En el modelo SV la amplitud del camino $|\alpha(c, l)|$ puede ser una distribución lognormal, de tipo Nakagami o de tipo Rayleigh. Para el canal UWB estándar se adopta la de tipo lognormal.

A partir de las pérdidas de propagación del canal y de los modelos de desvanecimiento lento y desvanecimientos a pequeña escala, se definen 4 modelos de 
canal según el escenario de aplicación de UWB denominados CM1, CM2, CM3, y CM4. CM1 es un modelo para escenario con visión directa (LOS) y una separación entre el transmisor y el receptor no superior a $4 \mathrm{~m}$. CM2 se sitúa en el mismo radio de cobertura pero para condiciones de visión indirecta (NLOS). CM3 es un modelo para situaciones NLOS con distancias entre transmisor y receptor de entre $4 \mathrm{~m}$ y $10 \mathrm{~m}$. En cambio CM4, es un modelo para escenarios con propagación multicamino y una importante dispersión por retardo, caracterizándose por valores mínimos de ensanchamiento temporal o delay spread de $25 \mathrm{~ns}$.

En la Tabla 3.1 se incluyen los principales parámetros de cada uno de estos modelos de canal, como son: el retardo medio por exceso $\left(\tau_{m}\right)$, el valor cuadrático medio del retardo $\left(\tau_{r m s}\right)$, el número de rayos medio dentro de un margen de $10 \mathrm{~dB}$ sobre el rayo con mayor energía $\left(N_{10 d B}\right)$, y el número medio de caminos que contienen el $85 \%$ de la energía transmitida en el canal $\left(N_{e}\right)$. También se incluyen las pérdidas por propagación de cada modelo de canal a un metro de distancia del transmisor $\left(P L_{0}(d B)\right)$.

Tabla 3.1. Parámetros característicos modelo de Canal UWB

\begin{tabular}{l|c|c|c|c}
\hline \hline Parámetros & CM1 & CM2 & CM3 & CM4 \\
\hline$\tau_{m}(\mathrm{~ns})$ & 5.0 & 9.9 & 15.9 & 30.1 \\
\hline$\tau_{r m s}(\mathrm{~ns})$ & 5.0 & 8.0 & 15.0 & 25.0 \\
\hline$N_{l O d B}$ & 12.5 & 15.3 & 24.9 & 41.2 \\
\hline$N_{e}$ & 20.8 & 33.9 & 64.7 & 123.3 \\
\hline$P L_{0}(\mathrm{~dB})$ & 47 & 50.5 & 50.5 & 50.5 \\
\hline \hline
\end{tabular}

\subsection{Análisis experimental y márgenes de protección en la coexistencia radio}

En este capítulo de esta Tesis Doctoral se ha evaluado la coexistencia de MB-OFDM UWB y WiMAX 802.16e en un escenario WPAN de tipo oficina. Este escenario es una sala de reuniones de la Universidad Politécnica de Valencia con dimensiones menores a $60 \mathrm{~m}^{2}$ y un mobiliario compuesto por madera, plástico y metales, tal y como se muestra en la fotografía de la Figura 3.2. A su vez, al estar situada la sala de reuniones en un edificio del campus universitario, existe la presencia de un punto de acceso $\mathrm{Wi}-\mathrm{Fi}$ a $2.4 \mathrm{GHz}$ y por lo tanto en el escenario de coexistencia WPAN bajo estudio. 


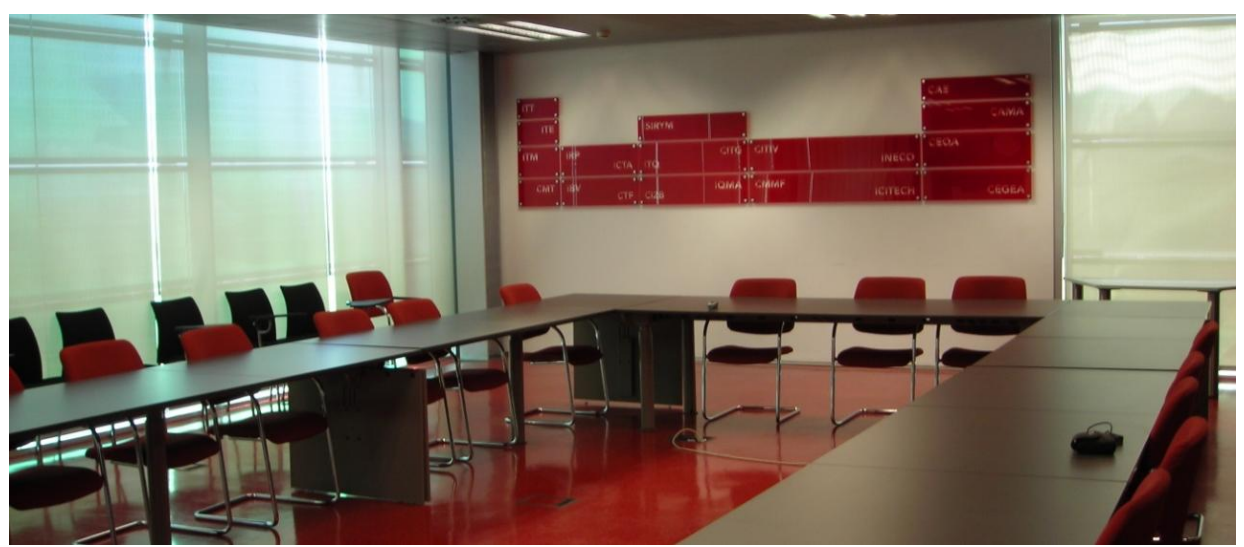

Figura 3.2. Sala de reuniones de la Universidad Politécnica de Valencia utilizada para la evaluación experimental de la coexistencia radio entre UWB y WiMAX.

En este escenario, se proponen diversas situaciones para evaluar la coexistencia entre un enlace WiMAX basado en IEEE 802.16e y un enlace MB-OFDM UWB basado en [ECMA368]. Como criterio para evaluar la calidad de los enlaces inalámbricos presentes en estos escenarios, se considera la magnitud del error del vector de señal recibida (EVM, Error Vector Magnitude), que es una figura de mérito en las comunicaciones de modulaciones digitales. El valor EVM es la medida del error cometido entre los símbolos medidos en recepción y aquellos esperados según el formato de modulación de la comunicación, como se observa en la Figura 3.3. El valor EVM permite inferir el comportamiento del receptor con anterioridad al cálculo de tasas de error de bit (BER, Bit Error Rate) de la comunicación.

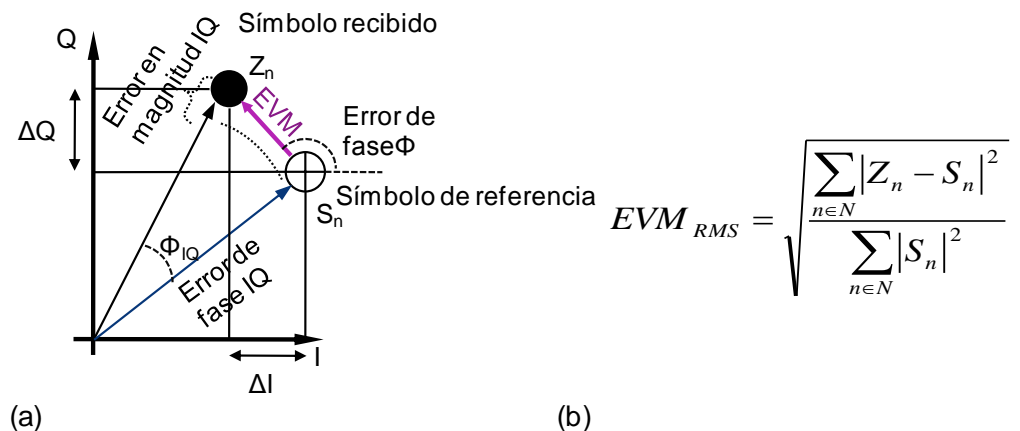

Figura 3.3. Definición de EVM en base a (a) representación grafica y a el (b) cálculo en función del símbolo recibido $Z_{\mathrm{n}} \mathrm{y}$ el símbolo de referencia $\mathrm{S}_{\mathrm{n}}$.

La relación directa entre los valores de EVM y los valores de BER de un sistema de comunicaciones es factible siempre que el ruido predominante en el sistema de 
comunicaciones sea de tipo Gaussiano [Sha06]. Sin embargo, en modulaciones QAM de tipo OFDM es bastante habitual evaluar la calidad de la constelación recibida en términos de EVM [Hu07]. Además, la evaluación de la calidad del enlace en términos de EVM permite obtener un análisis más completo de la distorsión del enlace en términos como la ganancia o el ruido de fase en la transmisión, [Has97], [Geo04].

\subsubsection{Escenario de medida experimental}

A continuación se detallan las características del escenario considerado en el estudio de la coexistencia radio entre MB-OFDM UWB y WiMAX 802.16e y el montaje experimental realizado. La distribución de los terminales UWB y el enlace WiMAX en la sala de reuniones se observa en la Figura 3.4. En la misma se incluye la localización de los diferentes elementos de transmisión y medida de este estudio. En la Figura 3.4 podemos observar en (a) el plano de la planta del edificio donde se sitúa la sala de reuniones y en (b) la distribución y localización de los equipos de trabajo para las medidas.

(a)

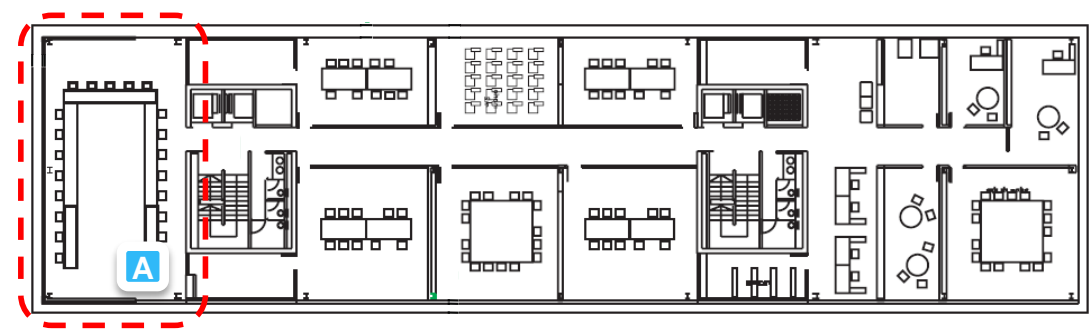

(b)

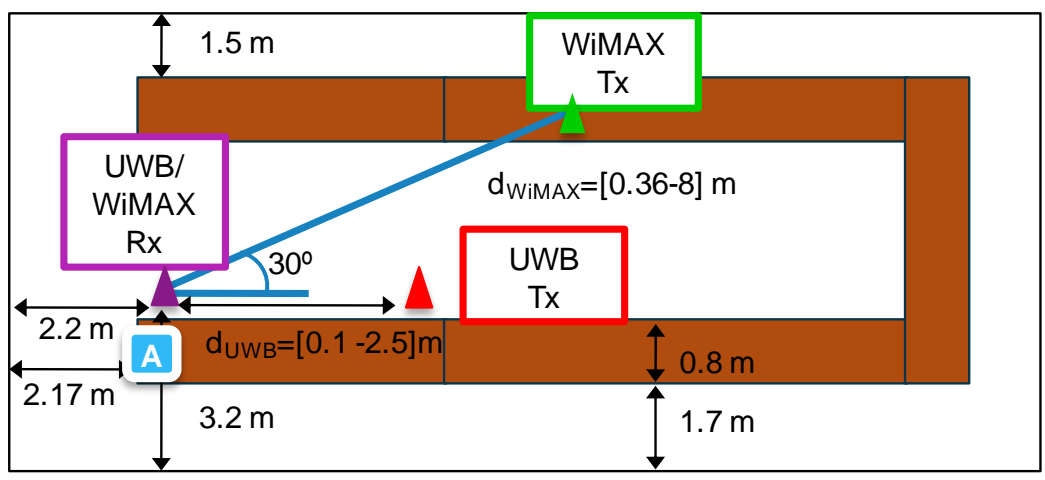

Figura 3.4. (a) Situación de la sala de reuniones. (b) Distribución y localización de los equipos usados en el estudio de coexistencia. La marca $\langle\mathrm{A}\rangle$ se usa como referencia entre el plano real y la distribución recreada.

En la Figura 3.4 (b) se observa como los enlaces inalámbricos tienen visión directa (LOS). Además, el enlace UWB bajo estudio no supera los $4 \mathrm{~m}$ de distancia entre transmisor y receptor, ante lo cual podemos asumir un modelo de canal UWB estándar 
del tipo CM1, con pérdidas de propagación en el primer metro del enlace de unos $42 \mathrm{~dB}$.

La separación angular ente ambos enlaces es de $30^{\circ}$, esta posición se ha escogido para evaluar el caso de mayor interferencia. A su vez, permite evaluar el comportamiento de terminales BWA para futuras sectorizaciones del acceso radio WiMAX ante la presencia de comunicaciones UWB.

El receptor WiMAX y el receptor UWB se ubican en el mismo punto para analizar la coexistencia en una situación de integración de servicios inalámbricos en futuros dispositivos, como por ejemplo ordenadores portátiles o terminales móviles. La altura de los transmisores y receptores se ha delimitado a un metro para poder estar situados por encima de la altura estándar de una mesa y una silla.

Las distancias de separación de cada enlace han sido escogidas atendiendo a los siguientes criterios. Las distancias UWB han sido escogidas de acuerdo con las especificaciones del estándar [ECMA368], en el cual se indica que para configuraciones de $200 \mathrm{Mbit} / \mathrm{s}$ en escenarios tipo oficina (multicamino) se pueden alcanzar transmisiones de hasta 3 m o 4 m [ECMA05]. En cambio, las distancias de separación del enlace WiMAX han sido escogidas para cumplir con las diferentes zonas que se establecen en la técnica de mitigación DAA para la banda de $3.4 \mathrm{GHz}$ a $4.2 \mathrm{GHz}$ [ECC120], como se comentó en el capítulo 2 de esta Tesis Doctoral.

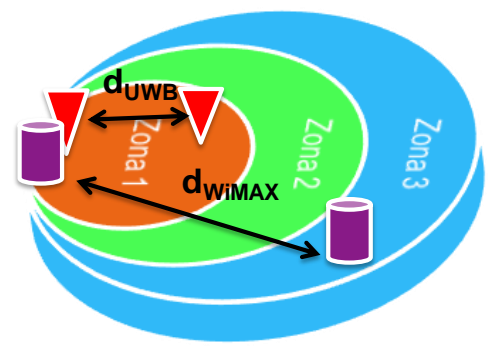

\begin{tabular}{|c|c|c|c|c|}
\hline \multirow{2}{*}{$d_{\text {WimAX }}$} & Zona1 & $0.36 \mathrm{~m}$ & $1 \mathrm{~m}$ & $1.7 \mathrm{~m}$ \\
\cline { 2 - 5 } & Zona2 & $3 \mathrm{~m}$ & $5 \mathrm{~m}$ \\
\cline { 2 - 5 } & Zona3 & \multicolumn{2}{|c|}{$8 \mathrm{~m}$} & $10 \mathrm{~m}$ \\
\hline \multicolumn{2}{|c|}{$\mathrm{d}_{\text {UWB }}$} & \multicolumn{2}{|c|}{$0.1 \mathrm{~m} \ldots 2.5 \mathrm{~m}$} \\
\hline
\end{tabular}

Figura 3.5. Distancias de los enlaces UWB y WiMAX y situación de las zonas 1, 2, 3 para técnicas de mitigación de la interferencia basadas en DAA.

Las distancias de separación del enlace WiMAX ( $\left.\mathrm{d}_{\text {WiMAX }}\right)$ y UWB $\left(\mathrm{d}_{\mathrm{UWB}}\right)$ escogidas se muestran en la Figura 3.5. Los valores $d_{\text {WiMAX }}$ se han determinado según la potencia de señal WiMAX recibida, permitiendo establecer umbrales de señal para la separación en zonas de exclusión, como se indica en [ECC120] para implementar DAA en futuros dispositivos UWB. Para determinar los valores $\mathrm{d}_{\mathrm{WiMAX}}$, se ha colocado en la posición de recepción del esquema de la Figura 3.4 (b) un transmisor WiMAX BWA basado en IEEE 802.16e, con una potencia de $200 \mathrm{~mW}$ o $23 \mathrm{dBm}$, siendo este el mayor nivel de potencia regulada para terminales BWA TS. A su vez, se ha usado una antena 
omnidireccional UWB para poder obtener el nivel de señal detectado tal y como se realizaría en un dispositivo UWB con técnicas DAA implementadas. Las medidas han sido capturadas mediante un analizador de espectros de radiofrecuencia.

Tras varias campañas de medidas en las cuales se han realizado las medidas con visión directa entre la antena y el TS BWA, se ha comprobado que en la sala de reuniones se cumplen los requisitos para poder establecer tres zonas de actuación de acuerdo con las técnicas DAA. Se ha delimitado el umbral de $-38 \mathrm{dBm}$ para las zonas 1 y 2 en una distancia de $3 \mathrm{~m}$, y el umbral de $-61 \mathrm{dBm}$ para las zonas 2 y 3 en una distancia cercana a $8 \mathrm{~m}$. Ante estos umbrales se ha escogido un intervalo de distancias en el enlace WiMAX de $\{0.36,1,1.7,3,5,8,10\}$ m para obtener resultados de acuerdo con las recomendaciones de implementación de técnicas de mitigación DAA en dispositivos UWB. A su vez, se pretende que estos resultados sean relevantes para las actividades de regulación en marcha en el ámbito de UWB. La elección de $0.36 \mathrm{~m}$ para la mínima distancia interferente WiMAX se realiza de acuerdo a resultados anteriores con terminales FWA y UWB mostrados en [Rah07].

Los equipos utilizados para implementar el enlace MB-OFDM UWB son desarrollos de evaluación de la compañía Wisair Ltd., en concreto el modelo DV9110. Estos módulos permiten generar señales UWB basadas en el estándar ECMA 368 en la banda obligatoria mandatory BG1 que comprende 3 subbandas de $528 \mathrm{MHz}$ de ancho de banda. Esto permite usar los 10 códigos de tiempo-frecuencia (TFC) disponibles según el estándar. Se ha optado por implementar un enlace MB-OFDM UWB con regímenes binarios de 53.3 Mbit/s y $200 \mathrm{Mbit} / \mathrm{s}$ de modulación QPSK. Para analizar el efecto de los diferentes TFC en el enlace UWB ante la presencia de WiMAX, se ha escogido un TFC que contemple el salto en frecuencia, TFC1, y otro código que no lo contemple y sea interferente con WiMAX 802.16e en la banda de $3.5 \mathrm{GHz}$, en este caso TFC5. Los principales parámetros del enlace MB-OFDM UWB se muestran en la Tabla 3.2.

TABLA 3.2. PARÁMETROS DEL ENLACE MB-OFDM UWB IMPLEMENTADO

\begin{tabular}{l|c|c}
\hline \hline TFC & TFC1 & TFC5 \\
\hline \multirow{2}{*}{ Banda de frecuencias } & Band group \#1, & Band \#1 \\
\cline { 2 - 3 } & $\{3.168-4.752 \mathrm{GHz}\}$ & $\{3.168-3.696 \mathrm{GHz}\}$ \\
\hline Frecuencia central & $3.96 \mathrm{GHz}$ & $3.432 \mathrm{GHz}$ \\
\hline Régimen binario & \multicolumn{2}{|c}{$53.3 \mathrm{Mbit} / \mathrm{s}, 200 \mathrm{Mbit} / \mathrm{s}$} \\
\hline EIRP & \multicolumn{2}{|c}{$-41.3 \mathrm{dBm} / \mathrm{MHz}$} \\
\hline \hline
\end{tabular}


En la Figura 3.6 se muestra el espectro de las señales MB-OFDM UWB generadas. Estos espectros han sido medidos antes de la antena transmisora del enlace MB-OFDM UWB para las configuraciones de TFC1 y TFC5 con $200 \mathrm{Mbit} / \mathrm{s}$.
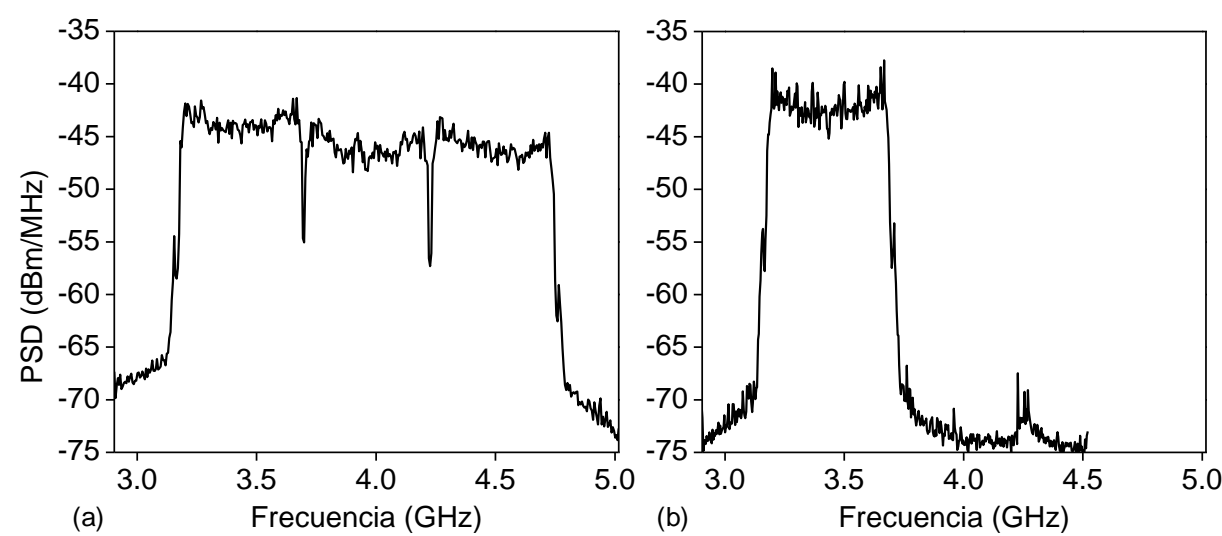

Figura 3.6. Espectro de la señal UWB generada para una configuración tipo (a) TFC1 y (b) TFC5 de $200 \mathrm{Mbit} / \mathrm{s}$. RBW = $1 \mathrm{MHz}$.

El enlace MB-OFDM UWB se ha implementado mediante 2 módulos transceptores UWB DV9110 y dos antenas omnidireccionales diseñadas para UWB de tipo patch In4Tel-omni con $0 \mathrm{dBi}$ de ganancia. Se ha utilizado un amplificador de bajo ruido y atenuador de radiofrecuencia para adecuar la señal UWB recibida al margen dinámico de los equipos de medida de EVM usados en este estudio. La medida del parámetro de calidad EVM se ha realizado mediante a un analizador digital de la señal en tiempo real, Agilent DSA 80000B junto con el software de análisis vectorial VSA 89600. Este DSA permite un análisis completo hasta $12 \mathrm{GHz}$ con $40 \mathrm{GS} / \mathrm{s}$.

El enlace WiMAX se ha implementado conforme al estándar IEEE 802.16e [IEEE80216e]. La señal WiMAX se ha generado conforme a un terminal interior TS BWA. Cabe recordar que BWA basa su acceso en OFDM escalable (SOFDMA) con modulación QPSK. La señal WiMAX 802.16e se ha generado en la banda de frecuencias de $3.5 \mathrm{GHz}(3.4 \mathrm{GHz}-3.8 \mathrm{GHz})$ de uso en Europa y Asia fundamentalmente [ECC07.02]. Los anchos de banda nominales de la señal WiMAX generada son $5 \mathrm{MHz}, 10 \mathrm{MHz}$ y $20 \mathrm{MHz}$ y con una modulación QPSK 1/2 CTC. Los principales parámetros de la enlace WiMAX implementado se muestran en la Tabla 3.3. 
Tabla 3.3. Parámetros del enlace WiMAX basado en IEEE 802. 16E

\begin{tabular}{l|c|c|c}
\hline \hline Frecuencia central & \multicolumn{3}{|c}{$3.5 \mathrm{GHz}$} \\
\hline Ancho de banda nominal & $5 \mathrm{MHz}$ & $10 \mathrm{MHz}$ & $20 \mathrm{MHz}$ \\
\hline Espaciado subportadoras & $5.46875 \mathrm{kHz}$ & $10.9375 \mathrm{kHz}$ & $21.875 \mathrm{kHz}$ \\
\hline $\begin{array}{l}\text { Duración de símbolo } \\
\text { (Ts=1 / Espaciado Subportadora) }\end{array}$ & $182.86 \mu \mathrm{s}$ & $102.85 \mu \mathrm{s}$ & $57.14 \mu \mathrm{s}$ \\
\hline Sobremuestreo & \multicolumn{3}{|c}{$24 / 28$} \\
\hline Periodo de guarda (G) & \multicolumn{3}{|c}{$1 / 8$} \\
\hline Modulación & \multicolumn{3}{|c}{ QPK (1/2 CTC) } \\
\hline Régimen binario en DL & $3.17 \mathrm{Mbit} / \mathrm{s}$ & $6.34 \mathrm{Mbit} / \mathrm{s}$ & $12.68 \mathrm{Mbit} / \mathrm{s}$ \\
\hline \hline
\end{tabular}

La señal WiMAX 802.16e se ha implementado mediante un generador de señales vectoriales (Agilent ESG 4483C $250 \mathrm{KHz}-6 \mathrm{GHz}$ ) y la sintetización de la señal en el programa Agilent N7615B Signal Studio. La señal se ha generado con una EIRP de $23 \mathrm{dBm}(200 \mathrm{~mW})$ a la salida de antena de acuerdo con IEEE 802.16 part 3 Radio Conformance Tests [IEEE80216b]. Esta potencia de señal se corresponde con la utilización de antenas WiMAX para terminales interiores de tipo plano, HUBER+SUHNER SPA 3500/65/9/0V, con 9 dBi de ganancia y la generación de la señal WiMAX con una potencia de $15 \mathrm{dBm}$ a la salida del ESG. La captura y análisis de los datos del enlace WiMAX se han realizado en un analizador de espectros digital R\&S FSQ40, que permite medidas de señales RF hasta $40 \mathrm{GHz}$. Este analizador implementa un software para la demodulación de señales WiMAX según IEEE 802.16d y IEEE 802.16e, lo que ha permitido evaluar los parámetros de calidad del enlace tales como la constelación recibida, la demodulación de la señal y el EVM asociado.

En la Figura 3.7 se muestran los espectros de la señal WiMAX 802.16e generada para los anchos de banda nominales (BW) de $5 \mathrm{MHz}, 10 \mathrm{MHz}$ y $20 \mathrm{MHz}$.

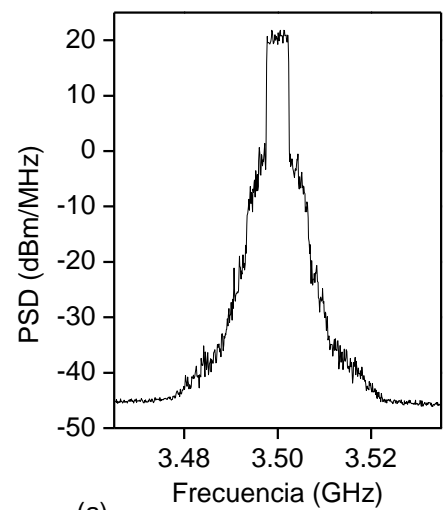

(a)

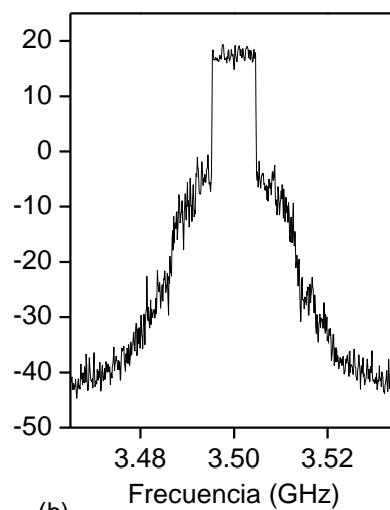

(b)

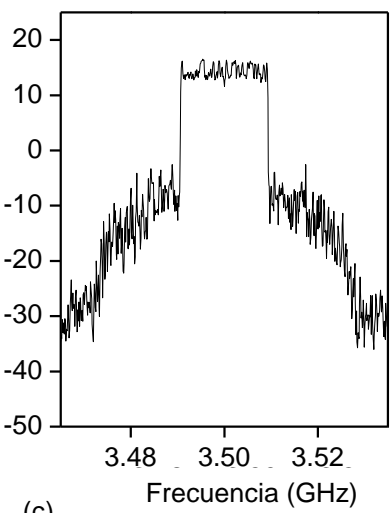

(c)

Figura 3.7. Espectros de la señal WiMAX 802.16e generada para un ancho de banda nominal de (a) $5 \mathrm{MHz}$, (b) $10 \mathrm{MHz}$ y (c) $20 \mathrm{MHz}$. RBW = 1 MHz. 
El estudio de la coexistencia se plantea en primer lugar contemplando el impacto de MB-OFDM UWB sobre un enlace WiMAX 802.16e y posteriormente, como el impacto de WiMAX 802.16e sobre el enlace MB-OFDM UWB.

En este estudio se ha utilizado la siguiente notación. WiMAX $10 \mathrm{MHz} \mathrm{BW}$, es un enlace WiMAX 802.16e configurado con un ancho de banda nominal de $10 \mathrm{MHz}$, donde el valor del ancho de banda nominal varía según el estudio experimental. MB-OFDM UWB TFC1 $53.3 \mathrm{Mbit} / \mathrm{s}$, es un enlace MB-OFDM UWB configurado con un código TFC de salto en frecuencia y un régimen binario de $53.3 \mathrm{Mbit} / \mathrm{s}$. Estos parámetros pueden variar según el estudio experimental. Por último, EVM UWB o EVM WiMAX, es el EVM medido sobre un enlace UWB o WiMAX, respectivamente.

\subsubsection{Prestaciones enlace WiMAX 802.16e en presencia de MB-OFDM UWB}

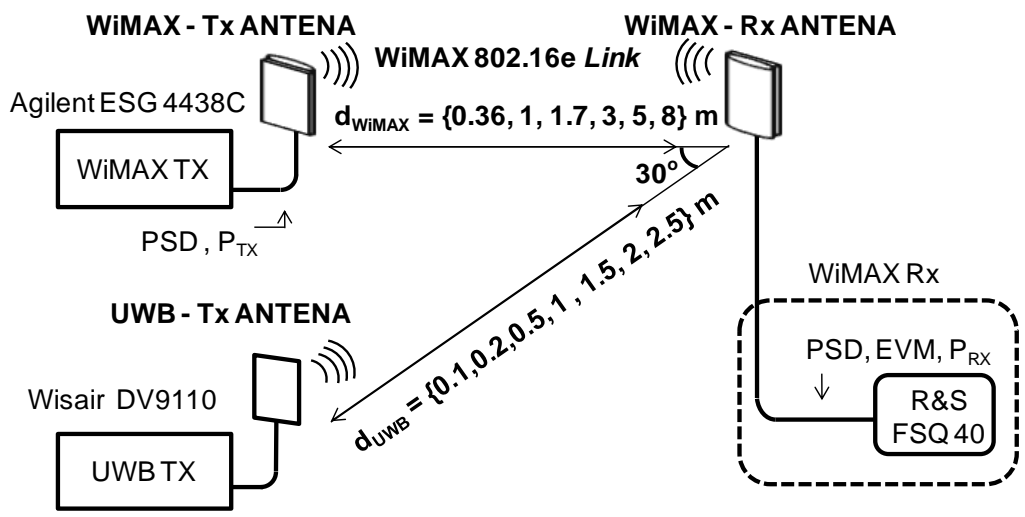

Figura 3.8. Montaje experimental para el estudio del enlace WiMAX 802.16e en presencia de un interferente MB-OFDM UWB.

Para evaluar las prestaciones de un enlace WiMAX 802.16e en presencia de un interferente MB-OFDM UWB se ha implementado el montaje experimental mostrado en la Figura 3.8. En este esquema se muestran los parámetros asociados con la distancia del enlace (link) WiMAX, la situación de los equipos de medida FSQ y ESG, así como la localización del módulo interferente MB-OFDM UWB.

En este escenario, la señal WiMAX se ve interferida por UWB al compartir el espectro RF de transmisión. Este solapamiento espectral se observa en la Figura 3.9, donde se muestra el espectro de la señal de un enlace WiMAX $10 \mathrm{MHz} \mathrm{BW}$ con $\mathrm{d}_{\text {WiMAX }}=5 \mathrm{~m}$ ante la interferencia de un enlace MB-OFDM UWB TFC1 situado a $\mathrm{d}_{\text {UWB }}$ de $0.10 \mathrm{~m}$ y $1 \mathrm{~m}$ respectivamente. En la Figura 3.9 se observa como aun ante el efecto de solapamiento espectral de UWB sobre una señal WiMAX el nivel de rechazo entre ambas señales es superior a $35 \mathrm{~dB}$, lo cual asegura la transmisión WiMAX [ECC120] [IEEE802.16e]. 

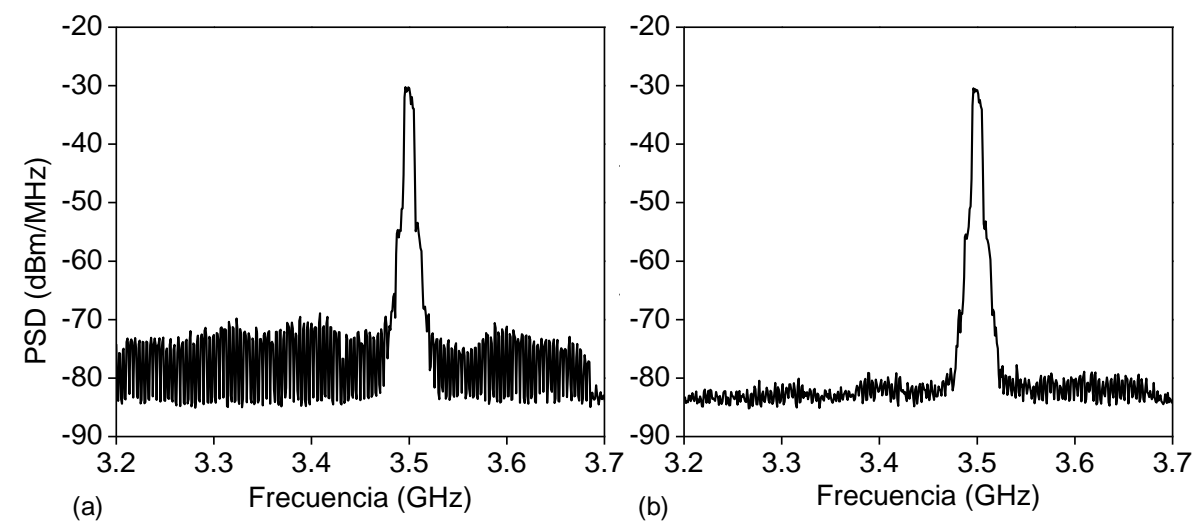

Figura 3.9. Espectros de la señal WiMAX 802.16e recibida para un ancho de banda nominal de $10 \mathrm{MHz}$ y $d_{\text {WiMAX }}=5 \mathrm{~m}$ en presencia de un interferente MB-OFDM UWB TFC1 situado en (a) $d_{U W B}=0.10 \mathrm{~m}$ y (b) $d_{U W B}=1 \mathrm{~m}$. RBW = 1 MHz.

En primer lugar se analiza las prestaciones del enlace WiMAX en el escenario bajo estudio. Para ello, se han generado señales WiMAX sin presencia de interferentes UWB activos. En la Tabla 3.4 se muestra el EVM medido para las diferentes distancias del enlace WiMAX en esta situación. A su vez, se ha medido la atenuación por propagación de la potencia WiMAX recibida. Por ejemplo, en una configuración WiMAX $10 \mathrm{MHz}$ BW se ha recibido una potencia de $-4.92 \mathrm{dBm}$ para $\mathrm{d}_{\text {WiMAX }}=0.36 \mathrm{~m},-15.20 \mathrm{dBm}$ para $\mathrm{d}_{\text {WiMAX }}=3 \mathrm{~m}$ y $-24.46 \mathrm{dBm}$ para $\mathrm{d}_{\text {WiMAX }}=8 \mathrm{~m}$.

TABLA 3.4. EVM MEDIDO PARA WIMAX SIN PRESENCIA UWB

\begin{tabular}{l|l|l|l|l|l|l}
\hline \hline \multirow{2}{*}{$\mathbf{B W}$} & \multicolumn{5}{|c}{$\mathbf{d}_{\text {WiMAX }}$} \\
\cline { 2 - 7 } & $\mathbf{0 . 3 6} \mathbf{~ m}$ & $\mathbf{1 ~} \mathbf{~ m}$ & $\mathbf{1 . 7} \mathbf{~ m}$ & $\mathbf{3 ~ m}$ & $\mathbf{5 ~ m}$ & $\mathbf{8 ~ m}$ \\
\hline $\mathbf{5} \mathbf{M H z}$ & $-15.59 \mathrm{~dB}$ & $-15.58 \mathrm{~dB}$ & $-15.56 \mathrm{~dB}$ & $-15.53 \mathrm{~dB}$ & $-15.50 \mathrm{~dB}$ & $-15.51 \mathrm{~dB}$ \\
\hline $\mathbf{1 0} \mathbf{M H z}$ & $-15.61 \mathrm{~dB}$ & $-15.62 \mathrm{~dB}$ & $-15.61 \mathrm{~dB}$ & $-15.60 \mathrm{~dB}$ & $-15.54 \mathrm{~dB}$ & $-15.54 \mathrm{~dB}$ \\
\hline $\mathbf{2 0} \mathbf{M H z}$ & $-15.64 \mathrm{~dB}$ & $-15.63 \mathrm{~dB}$ & $-15.60 \mathrm{~dB}$ & $-15.62 \mathrm{~dB}$ & $-15.60 \mathrm{~dB}$ & $-15.58 \mathrm{~dB}$ \\
\hline \hline
\end{tabular}

Según el estándar WiMAX 802.16e, el valor de EVM que garantiza una comunicación WiMAX con modulación QPSK 1/2 es - 15 dB. Los valores mostrados en la Tabla 3.4, indican que el enlace WiMAX implementado en este escenario posibilita la comunicación WiMAX sin presencia de interferente MB-OFDM UWB.

A continuación se muestran el EVM medido para configuraciones WiMAX con $\mathrm{d}_{\text {WiMAX }}$ de $0.36 \mathrm{~m}, 3 \mathrm{~m}$ y $8 \mathrm{~m}$ ante la presencia de un interferente MB-OFDM UWB. En la Figura 3.10 se ha mostrado el impacto de las diferentes configuraciones MB-OFDM UWB con diferente régimen binario y TFC sobre un enlace WiMAX de $\mathrm{d}_{\text {WiMAX }}=0.36 \mathrm{~m}$. Para esta distancia tan corta entre terminales WiMAX, las diferencias 
entre las diferentes configuraciones de ancho de banda nominal WiMAX son mínimas. Se observa que para una misma configuración WiMAX, el efecto de la presencia de las diferentes configuraciones del interferente UWB degrada de la misma forma el enlace WiMAX. Por ejemplo, para un enlace WiMAX $10 \mathrm{MHz} B W$ con $\mathrm{d}_{\text {WiMAX }}=0.36 \mathrm{~m}$, el valor de EVM sin presencia de interferente UWB es $-15.61 \mathrm{~dB}$, ante la presencia de MB-OFDM UWB TFC5 $53.3 \mathrm{Mbit} / \mathrm{s}$ a $\mathrm{d}_{\mathrm{UWB}}=0.1 \mathrm{~m}$ es de $-15.38 \mathrm{~dB}$, y para UWB TFC5 $200 \mathrm{Mbit} / \mathrm{s}$ a la misma distancia es de $-15.388 \mathrm{~dB}$.
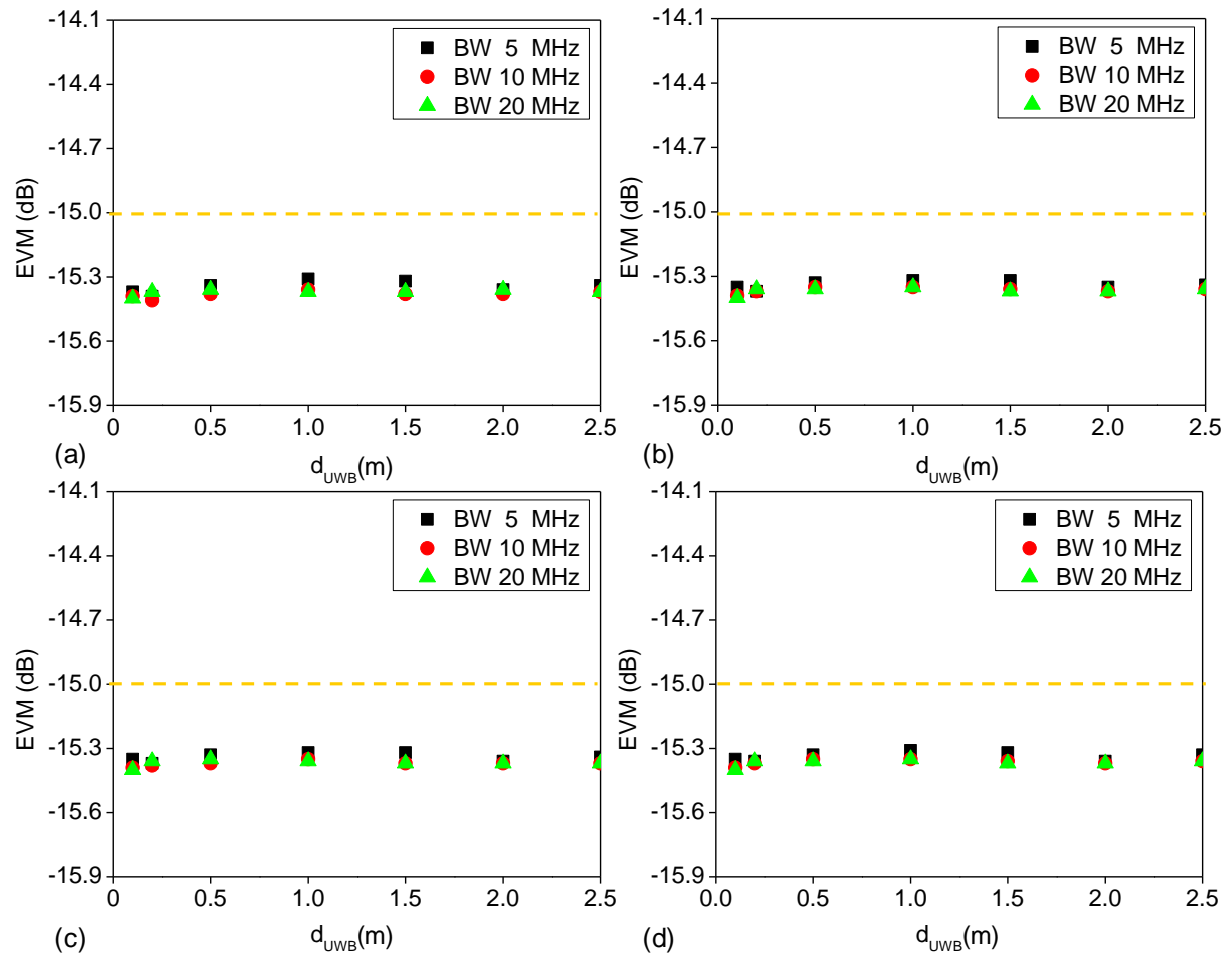

Figura 3.10. EVM medido para un enlace WiMAX 5 MHz, 10 MHz y 20 MHz BW con $d_{\text {WiMAX }}=0.36 \mathrm{~m}$ ante la presencia de MB-OFDM UWB (a) TFC1, (b) TFC5 53.3 Mbit/s, y para MB-OFDM UWB (c) TFC1 y (d) TFC5 $200 \mathrm{Mbit} / \mathrm{s}$. 

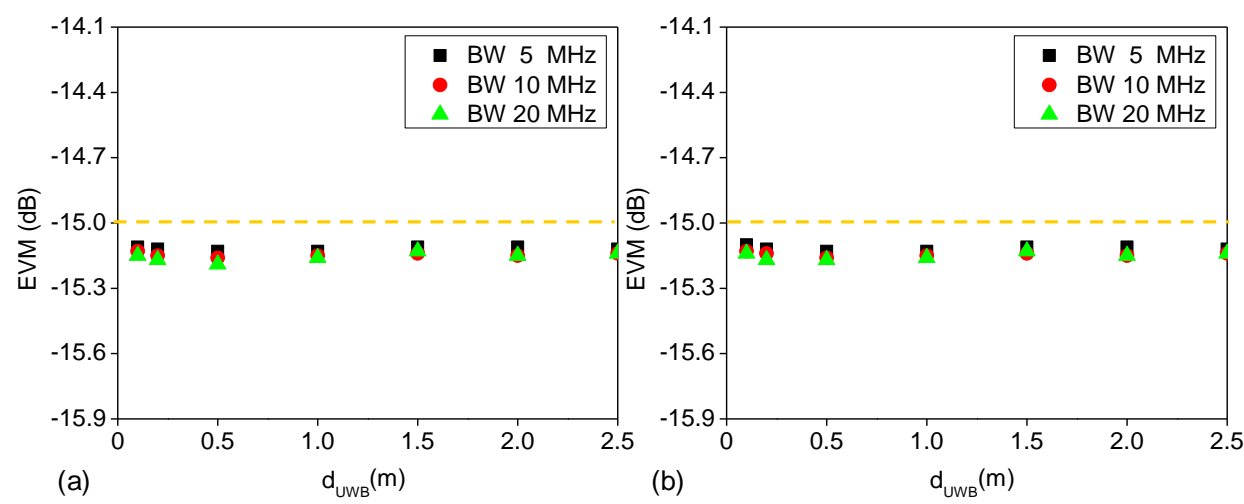

Figura 3.11. EVM medido para un enlace WiMAX 5 MHz, 10 MHz y 20 MHz BW con $d_{\text {WiMAX }}=3 \mathrm{~m}$ ante la presencia de MB-OFDM UWB (a) TFC1 y (b) TFC5 $200 \mathrm{Mbit} / \mathrm{s}$.
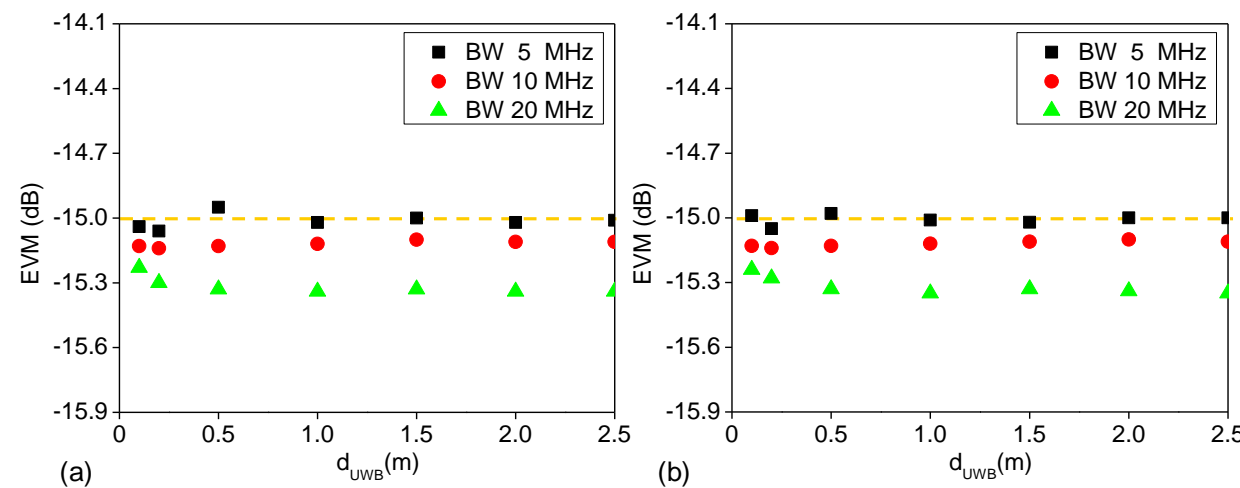

Figura 3.12. EVM medido para un enlace WiMAX de $5 \mathrm{MHz}, 10 \mathrm{MHz}$ y $20 \mathrm{MHz} B W$ con $\mathrm{d}_{\text {WiMAX }}=8 \mathrm{~m}$ ante la presencia de MB-OFDM UWB (a) TFC1 y (b) TFC5 $200 \mathrm{Mbit} / \mathrm{s}$.

La Figura 3.11 y Figura 3.12 muestran las prestaciones del enlace WiMAX para $\mathrm{d}_{\text {WiMAX }}=3 \mathrm{~m}$ y $\mathrm{d}_{\text {WiMAX }}=8 \mathrm{~m}$ ante la presencia de un interferente MB-OFDM UWB configurado con TFC1 y TFC5 $200 \mathrm{Mbit} / \mathrm{s}$. Los valores EVM WiMAX ante la presencia de un interferente MB-OFDM UWB 53.3 Mbit/s no han sido considerados debido a que la penalización para las dos configuraciones de régimen binario para el interferente MB-OFDM UWB no difieren en más de 0.03 dB EVM, como se observa comparando el EVM mostrado en la Figura 3.10 (a) y (c).

En la Figura 3.10, Figura 3.11 y Figura 3.12 se observa como el aumento de la distancia en el enlace WiMAX produce una disminución del EVM. A su vez, se observa como en la zona 3 según DAA, es decir para $d_{\text {WiMAX }} \geq 8 \mathrm{~m}$, la configuración con menor ancho de banda nominal se vuelve más vulnerable a la interferencia MB-OFDM UWB 
debido tanto a la distancia del enlace WiMAX como a su menor ancho de banda. Esta relación entre el EVM WiMAX y el ancho de banda nominal WiMAX se observa claramente en la Figura 3.11, donde conforme aumenta el ancho de banda nominal se obtienen mejores resultados de EVM WiMAX. No obstante, todas las configuraciones WiMAX evaluadas proporcionan resultados por debajo del umbral de $-15 \mathrm{~dB}$ EVM WiMAX, lo cual garantiza la comunicación sobre el enlace WiMAX implementado.

En la Figura 3.13 se muestra el EVM WiMAX medido para diferentes anchos de banda nominales. Se han evaluado las prestaciones del enlace WiMAX ante un interferente MB-OFDM UWB TFC5 $200 \mathrm{Mbit} / \mathrm{s}$, configuración en la cual el solapamiento espectral de UWB sobre la señal WiMAX es continuo.

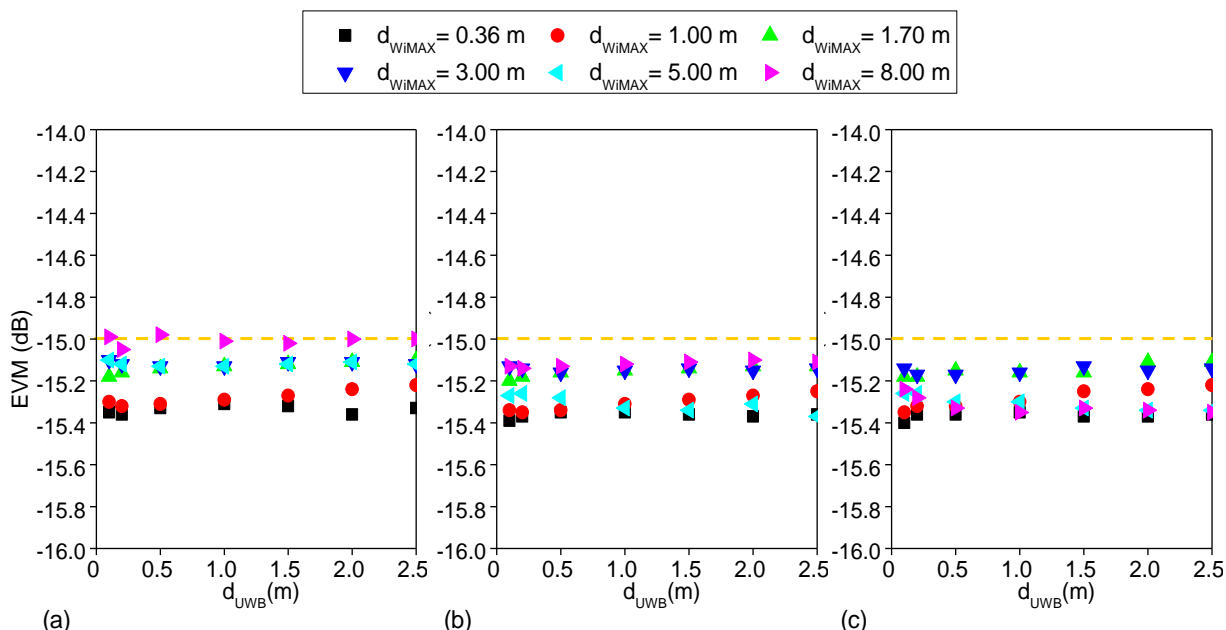

(a)

(b)

(c)

Figura 3.13. EVM medido para WiMAX (a) 5 MHz, (b) 10 MHz y (c) 20 MHz BW en presencia de MB-OFDM UWB TFC5 $200 \mathrm{Mbit} / \mathrm{s}$.

El EVM WiMAX mostrado en la Figura 3.13 indica que la comunicación sobre el enlace WiMAX implementado es viable en todos los casos. Sin embargo, para el caso de un enlace WiMAX $5 \mathrm{MHz}$ BW para $\mathrm{d}_{\text {WiMAX }}>5 \mathrm{~m}$, el interferente MB-OFDM UWB debe estar situado en un margen de seguridad mayor de $2.5 \mathrm{~m}$ como se observa en la Figura 3.13 (a). Los valores EVM WiMAX indican que la degradación sufrida en el enlace WiMAX ante la presencia de UWB no es superior a $0.5 \mathrm{~dB}$ EVM. Por ejemplo, para un enlace WiMAX $10 \mathrm{MHz} \mathrm{BW}$ en $\mathrm{d}_{\text {WiMAX }}=5 \mathrm{~m}$, el valor de EVM sin presencia UWB es de $-15.64 \mathrm{~dB}$, y ante la presencia de UWB TFC1 a una distancia de $\mathrm{d}_{\mathrm{UWB}}=0.5 \mathrm{~m}$, el valor de EVM WiMAX disminuye a $-15.28 \mathrm{~dB}$.

La evaluación realizada en las diferentes configuraciones de enlace WiMAX, indica que ante un aumento del ancho de banda nominal de la configuración WiMAX la 
calidad del enlace mejora y es más robusto a la presencia de interferentes MB-OFDM UWB. Por ejemplo, para un enlace WiMAX $5 \mathrm{MHz} \mathrm{BW}$, se observa una penalización de hasta $0.5 \mathrm{~dB}$ por la presencia de un interferente MB-OFDM UWB en un área menor de $2.5 \mathrm{~m}$.

\subsubsection{Prestaciones enlace MB-OFDM UWB en presencia de WiMAX 802.16e}

Para evaluar el comportamiento de un enlace MB-OFDM UWB en presencia de un interferente WiMAX 802.16e, se ha implementado el montaje experimental mostrado en la Figura 3.14. El enlace UWB está compuesto por módulos DV9110, y la señal UWB recibida es analizada mediante un DSA. La generación de las señales UWB y WiMAX que conforman el enlace MB-OFDM UWB y el interferente WiMAX 802.16e, se han implementado en base a los parámetros recogidos por la Tabla 3.2 y Tabla 3.3, respectivamente. En el esquema de la Figura 3.14 se muestran las distancias del enlace (link) UWB, y las distancias del interferente WiMAX acordes con las zonas DAA. En la recepción de la señal UWB se ha utilizado un amplificador de bajo ruido para adecuar la señal UWB recibida al margen dinámico disponible en el DSA, que permite evaluar el EVM asociado al enlace radio UWB.

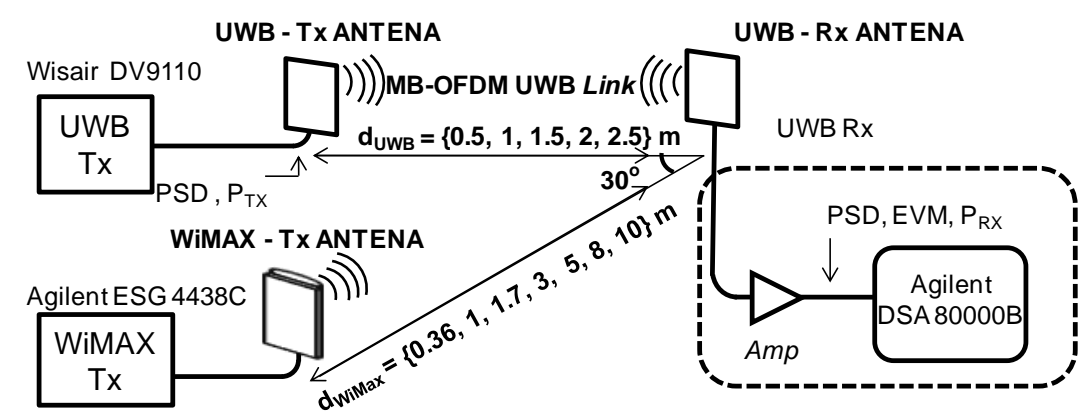

Figura 3.14. Montaje experimental para el estudio del enlace MB-OFDM UWB en coexistencia con WiMAX.

La Tabla 3.5 muestra el EVM UWB medido para el enlace MB-OFDM UWB TFC1 y TFC5 con diferentes régimen binario sin presencia de interferente WiMAX. Cabe destacar que el umbral EVM UWB para asegurar la comunicación entre dispositivos UWB es -14.4 dB según [ECMA368]. 
TABLA 3.5. EVM MEDIDO PARA MB-OFDM UWB SIN PRESENCIA WIMAX

\begin{tabular}{|c|c|c|c|c|c|c|}
\hline \multirow{2}{*}{\multicolumn{2}{|c|}{ TFC y régimen binario }} & \multicolumn{5}{|c|}{ "Distancia enlace $d_{\text {UWB }}$} \\
\hline & & $0.5 \mathrm{~m}$ & $1 \mathrm{~m}$ & $1.5 \mathrm{~m}$ & $2 \mathrm{~m}$ & $2.5 \mathrm{~m}$ \\
\hline \multirow{2}{*}{ TFC1 } & $53.3 \mathrm{Mbit} / \mathrm{s}$ & $-22.05 \mathrm{~dB}$ & $-21.93 \mathrm{~dB}$ & $-21.50 \mathrm{~dB}$ & $-20.62 \mathrm{~dB}$ & $-21.18 \mathrm{~dB}$ \\
\hline & $200 \mathrm{Mbit} / \mathrm{s}$ & $-21.72 \mathrm{~dB}$ & $-21.62 \mathrm{~dB}$ & $-21.27 \mathrm{~dB}$ & $-21.21 \mathrm{~dB}$ & $-21.24 \mathrm{~dB}$ \\
\hline \multirow{2}{*}{ TFC5 } & $53.3 \mathrm{Mbit} / \mathrm{s}$ & $-22.89 \mathrm{~dB}$ & $-22.63 \mathrm{~dB}$ & $-22.05 \mathrm{~dB}$ & $-21.49 \mathrm{~dB}$ & $-21.47 \mathrm{~dB}$ \\
\hline & $200 \mathrm{Mbit} / \mathrm{s}$ & $-22.39 \mathrm{~dB}$ & $-22.24 \mathrm{~dB}$ & $-21.75 \mathrm{~dB}$ & $-21.02 \mathrm{~dB}$ & $-20.67 \mathrm{~dB}$ \\
\hline
\end{tabular}

En la Figura 3.15 se observa el espectro recibido para una configuración MB-OFDM UWB TFC1 y TFC5 $53.3 \mathrm{Mbit} / \mathrm{s}$ con un enlace de $\mathrm{d}_{\mathrm{UWB}}=1 \mathrm{~m}$, ante la presencia de un interferente WiMAX $10 \mathrm{MHz}$ BW situado a $\mathrm{d}_{\text {WiMAX }}=5 \mathrm{~m}$. Se puede observar como existe un solapamiento espectral entre MB-OFDM UWB y WiMAX 802.16e.

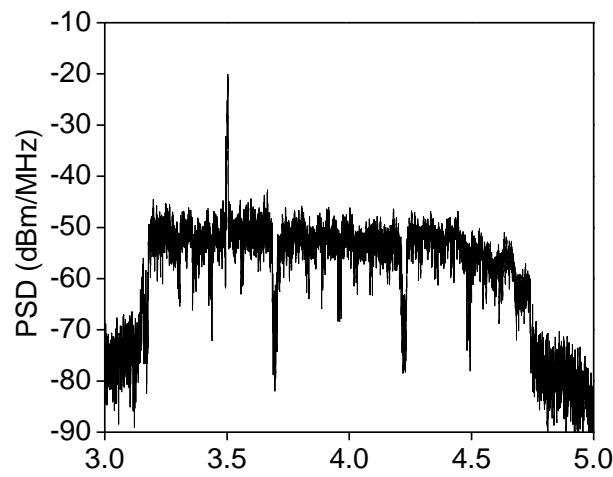

(a) Frecuencia $(\mathrm{GHz})$

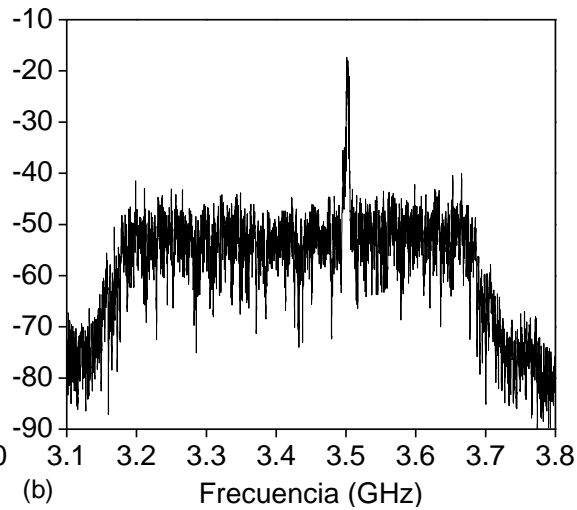

Figura 3.15. Espectros recibidos MB-OFDM UWB (a) TFC1 y (b) TFC5

53.3 Mbit/s para $d_{U W B}=1 \mathrm{~m}$ ante la presencia de un interferente WiMAX $10 \mathrm{MHz}$ BW situado a $\mathrm{d}_{\text {WiMAX }}=5 \mathrm{~m}$. $\mathrm{RBW}=1 \mathrm{MHz}$. 

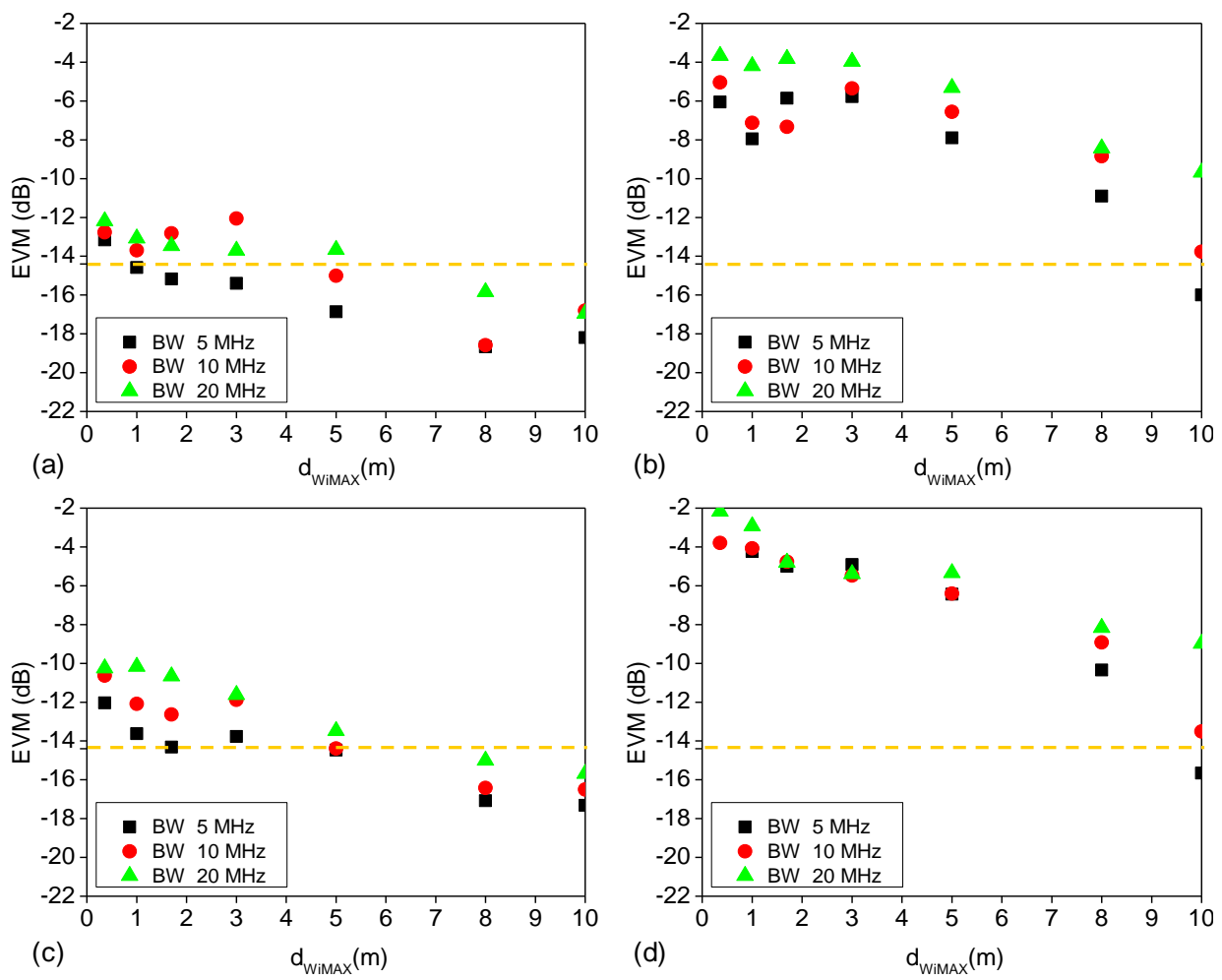

Figura 3.16. EVM medido para un enlace MB-OFDM UWB (a) TFC1 y (b) TFC 5 $53.3 \mathrm{Mbit} / \mathrm{s}$, y (c) TFC1, (d) TFC $5200 \mathrm{Mbit} / \mathrm{s}$ con $\mathrm{d}_{\mathrm{UWB}}=\mathbf{0 . 5} \mathrm{m}$ para diferentes configuraciones WiMAX interferente. 

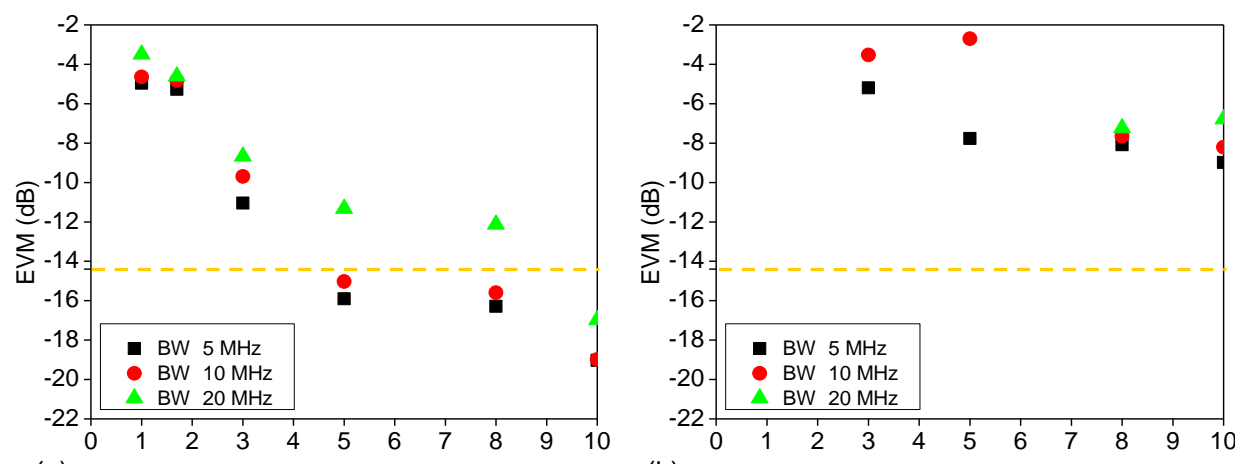

(a)

(b)
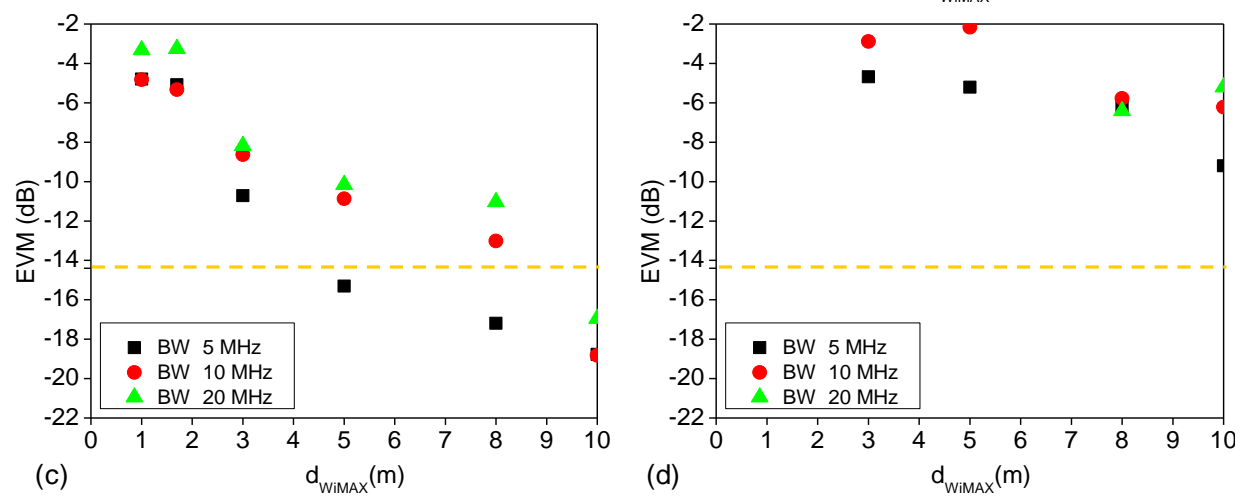

Figura 3.17. EVM medido para un enlace MB-OFDM UWB (a) TFC1 y (b) TFC 5 53.3 Mbit/s, y (c) TFC1, (d) TFC $5200 \mathrm{Mbit} / \mathrm{s}$ con $\mathrm{d}_{\mathrm{UWB}}=1 \mathrm{~m}$ para diferentes configuraciones WiMAX interferente. 

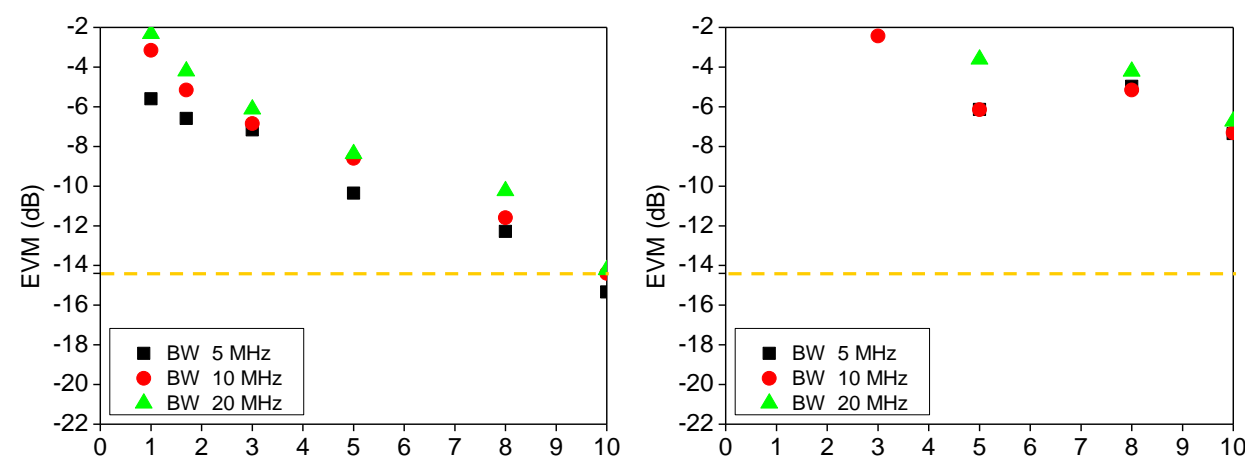

(a)

(b)
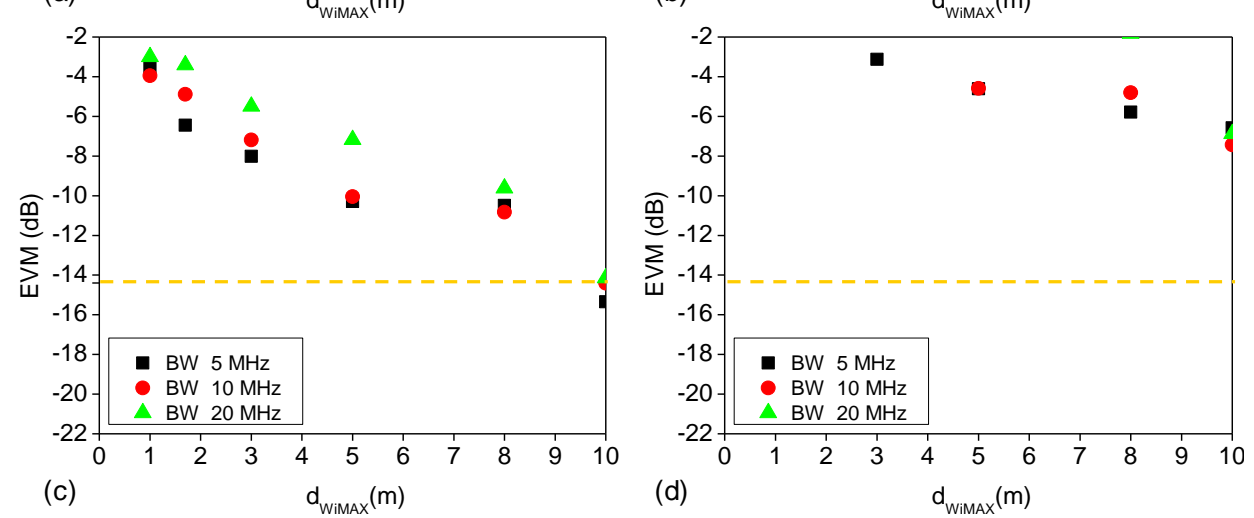

Figura 3.18. EVM medido para un enlace MB-OFDM UWB (a) TFC1 y (b) TFC 5 $53.3 \mathrm{Mbit} / \mathrm{s}$, y (c) TFC1, (d) TFC $5200 \mathrm{Mbit} / \mathrm{s}$ con $d_{\mathrm{UWB}}=2 \mathrm{~m}$ para diferentes configuraciones WiMAX interferente.

En la Figura 3.16, Figura 3.17 y Figura 3.18 se representa el umbral EVM UWB como una línea discontinua con valor $-14.4 \mathrm{~dB}$.

El EVM medido para el enlace UWB en presencia de WiMAX, se muestra en la Figura 3.16 para una distancia de enlace UWB $\mathrm{d}_{\mathrm{UWB}}=0.5 \mathrm{~m}$, en la Figura 3.17 para $\mathrm{d}_{\mathrm{UWB}}=1 \mathrm{~m}$ y en la Figura 3.18 para $\mathrm{d}_{\mathrm{UWB}}=2 \mathrm{~m}$. A su vez, en estas figuras se muestra el EVM UWB medido para cada una de las 4 configuraciones del enlace MB-OFDM UWB, es decir TFC1 y TFC5 con $53.3 \mathrm{Mbit} / \mathrm{s}$ y $200 \mathrm{Mbit} / \mathrm{s}$. A partir de los valores EVM UWB mostrados en estas figuras, se pueden inferir que la configuración TFC5 es la que sufre una mayor degradación por la presencia del interferente WiMAX.

Con respecto al impacto de las diferentes configuraciones WiMAX, se ha observado como al reducir el ancho de banda nominal WiMAX, aumenta las prestaciones del enlace MB-OFDM UWB. Por ejemplo, para un enlace MB-OFDM UWB TFC1 $53.3 \mathrm{Mbit} / \mathrm{s}$ con $\mathrm{d}_{\mathrm{UWB}}=1 \mathrm{~m}$, la comunicación UWB es factible si el 
interferente WiMAX está situado a $\mathrm{d}_{\text {WiMAX }}>5 \mathrm{~m}$. Sin embargo, para el mismo enlace UWB con $200 \mathrm{Mbit} / \mathrm{s}$ el margen de seguridad para comunicaciones UWB aumenta hasta $\mathrm{d}_{\text {WiMAX }}>8 \mathrm{~m}$ ante cualquier interferente WiMAX. Por ejemplo, la penalización sufrida por un enlace MB-OFDM UWB TFC1 $200 \mathrm{Mbit} / \mathrm{s} \mathrm{d}_{\mathrm{UWB}}=1 \mathrm{~m}$ ante un interferente WiMAX $10 \mathrm{MHz}$ BW situado a $\mathrm{d}_{\text {WiMAX }}=5 \mathrm{~m}$ es de $10 \mathrm{~dB}$ EVM UWB, ya que se obtiene -21.62 dB EVM UWB sin presencia WiMAX y -10.36 dB EVM UWB para la presencia del interferente WiMAX.
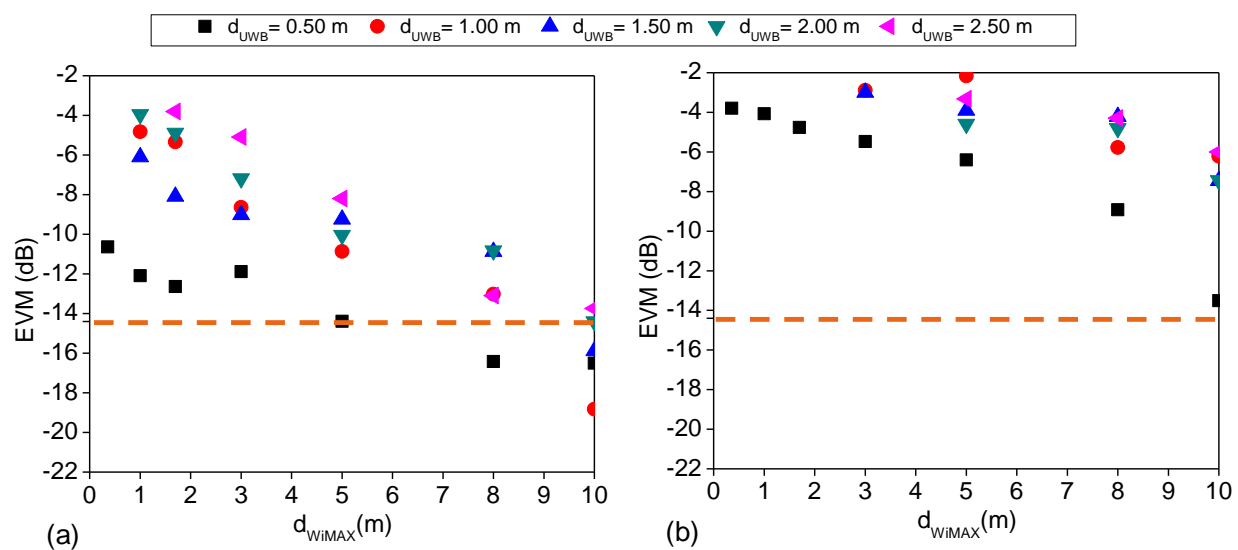

Figura 3.19. EVM medido para un enlace MB-OFDM UWB (a) TFC1 y (b) TFC 5 $200 \mathrm{Mbit} / \mathrm{s}$ en presencia de interferente WiMAX 10 MHz BW.

Los valores EVM UWB de la Figura 3.19 muestran la variación del enlace MB-OFDM UWB conforme a $\mathrm{d}_{\mathrm{UWB}}$ ante la presencia de un interferente WiMAX $10 \mathrm{MHz}$ BW. La Figura 3.19 indica que para un interferente WiMAX fijo, los valores EVM UWB empeoran al aumentar $\mathrm{d}_{\mathrm{UWB}}$. Para el caso de un enlace MB-OFDM UWB TFC1 $200 \mathrm{Mbit} / \mathrm{s}$ en presencia de un interferente WiMAX $10 \mathrm{MHz} \mathrm{BW}$ la comunicación UWB es viable si el interferente está situado en $\mathrm{d}_{\mathrm{WiMAX}} \geq 10 \mathrm{~m}$. Por ejemplo, para un enlace MB-OFDM UWB TFC1 $200 \mathrm{Mbit} / \mathrm{s}$ con $\mathrm{d}_{\mathrm{UwB}}=1 \mathrm{~m}$ sin interferencia, el valor EVM es $-21.62 \mathrm{~dB}$ y ante la presencia de un interferente WiMAX $10 \mathrm{MHz} B W$ situado en $\mathrm{d}_{\text {WiMAX }}=5 \mathrm{~m}$ disminuye hasta $-15.64 \mathrm{~dB}$ EVM UWB.

Para poder analizar la interferencia que introduce WiMAX sobre UWB, se ha evaluado la EVM presente en cada subportadora UWB. La Figura 3.20 muestra el EVM medido por cada subportadora. En la misma figura se representa mediante línea continua el valor medio de EVM por subportadora, el EVM de las subportadoras de tipo data de MB-OFDM UWB se representa con asteriscos azules, el EVM de las subportadoras piloto mediante círculos rojos y el EVM de las subportadoras de guarda mediante cuadrados violetas. Los valores EVM se miden considerando más de un símbolo OFDM, por ello aparecen múltiples valores para una misma subportadora. 

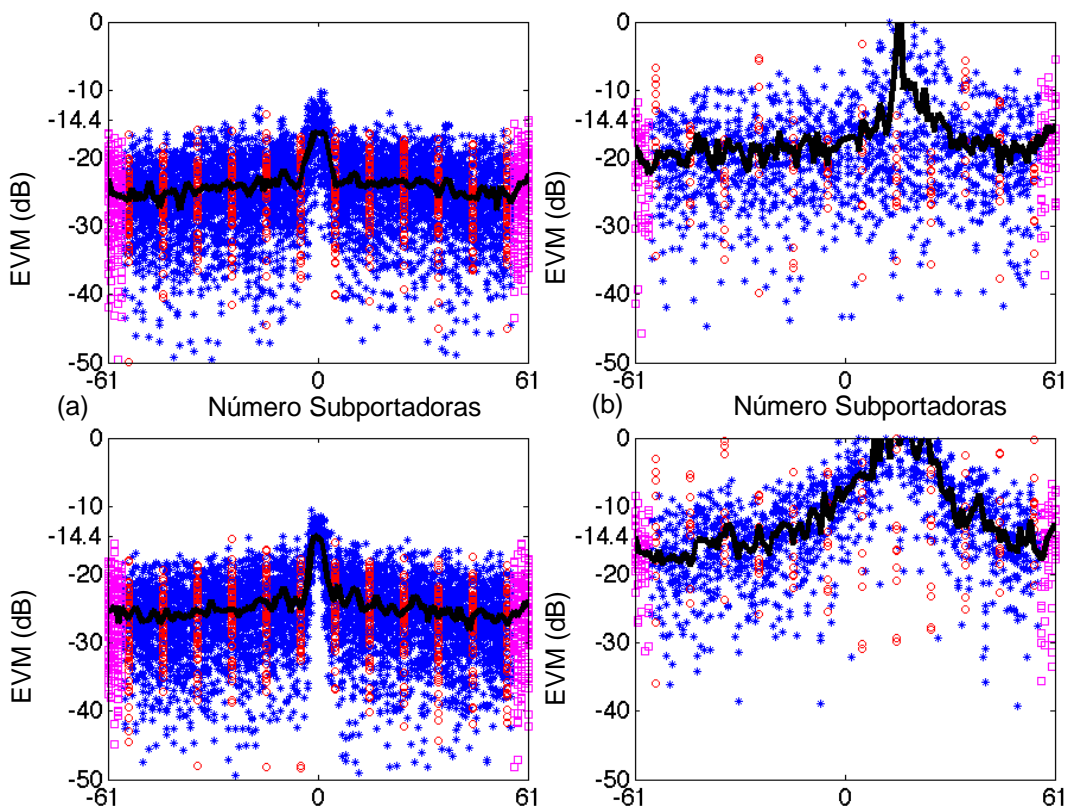

(b)

Número Subportadoras

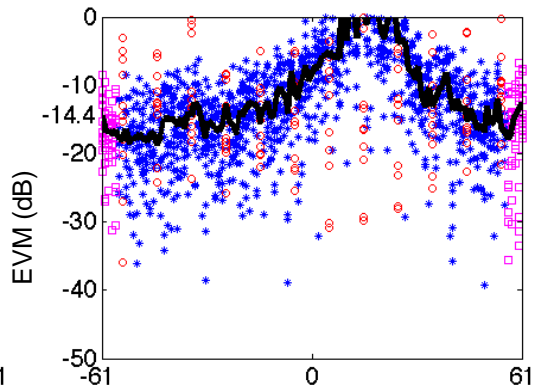

(d) Número Subportadoras

Número Subportadoras

Figura 3.20. EVM medido por subportadora MB-OFDM UWB TFC1 $53.3 \mathrm{Mbit} / \mathrm{s}$ (a) sin WiMAX, (b) con WiMAX $5 \mathrm{MHz}$ BW sito en $\mathrm{d}_{\text {WiMAX }}=1 \mathrm{~m}$; y para MB-OFDM UWB TFC5 53.3 Mbit/s (c) sin WiMAX y con (d) WiMAX 5 MHz BW sito en $d_{\text {WiMAX }}=1 \mathrm{~m}$.

Los valores EVM expuestos en la Figura 3.20 indican que la presencia de WiMAX no solo se traduce en una reducción del valor medio EVM del enlace MB-OFDM UWB, sino que también se traduce en la degradación de un número concreto de subportadoras del enlace MB-OFDM UWB como se observa en Figura 3.20 (b) y (d). A su vez, se observa de forma gráfica como este efecto sobre las subportadoras de cada símbolo OFDM recibido es mayor para la configuración MB-OFDM UWB TFC5 que para MB-OFDM UWB TFC1, como mostraban los valores EVM UWB expuestos anteriormente. 

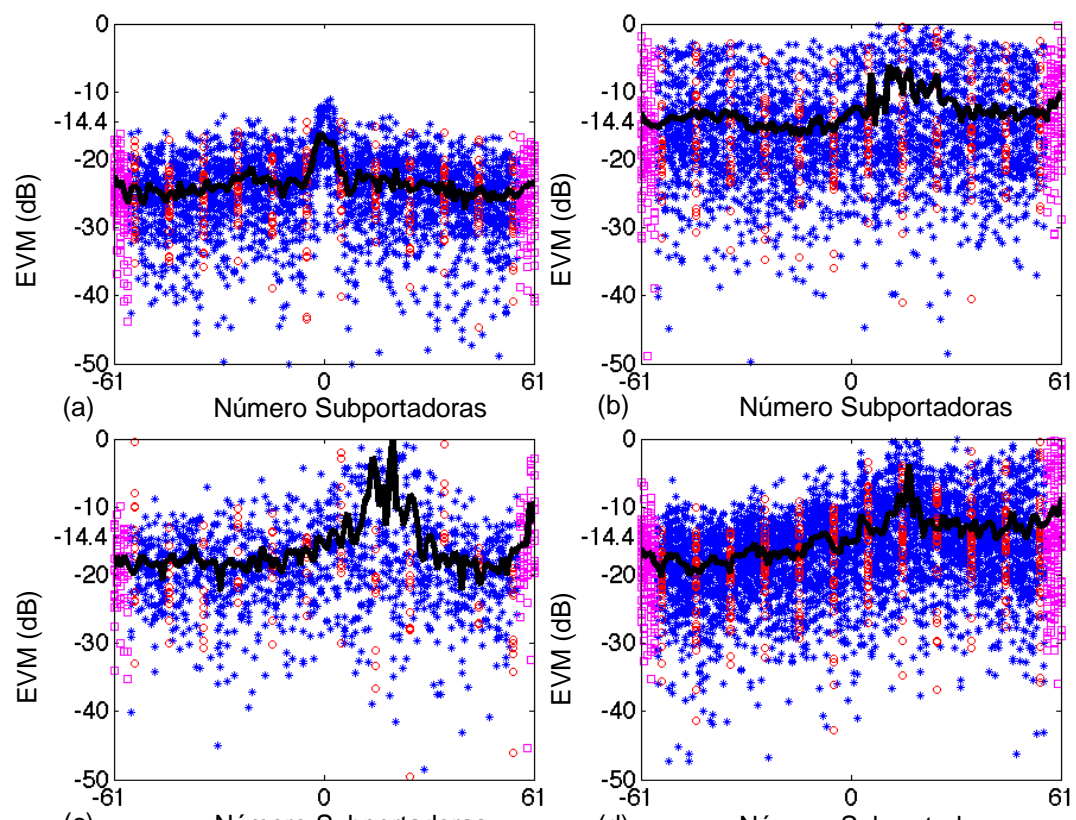

(c)

Número Subportadoras

(d)

Número Subportadoras

Figura 3.21. EVM medido por subportadora MB-OFDM UWB TFC1 $200 \mathrm{Mbit} / \mathrm{s}$ (a) sin WiMAX, (b) con WiMAX $5 \mathrm{MHz}$ BW sito en $\mathrm{d}_{\text {WiMAX }}=1 \mathrm{~m}$; para MB-OFDM UWB TFC1 53.3 Mbit/s con (c) WiMAX $10 \mathrm{MHz}$ BW sito en $\mathrm{d}_{\text {WiMAX }}=1$ m y (d) WiMAX $5 \mathrm{MHz} B W$ sito en $\mathrm{d}_{\text {WiMAX }}=3 \mathrm{~m}$.

La Figura 3.21 (a) y (b) muestra el EVM medido en cada subportadora para un enlace con configuración MB-OFDM UWB TFC1 $200 \mathrm{Mbit} / \mathrm{s}$ ante la presencia de WiMAX. Se observa como un rango concreto de subportadoras se ven degradadas de forma más intensa ante la presencia del interferente WiMAX, al igual que se muestra en la Figura 3.20 (b).También se observa la variación de EVM UWB para las subportadoras al aumentar el BW del interferente WiMAX en la Figura 3.21 (c), siendo más intensa la degradación pero localizada en el mismo rango de subportadoras. En la Figura 3.21 (d) se muestra el impacto al aumentar la distancia del interferente WiMAX. Al comparar la Figura 3.21 (c) y (d) se observa como al aumentar la separación del interferente WiMAX disminuye la degradación del EVM UWB.

En esta evaluación experimental, se han mostrado los márgenes de distancia o márgenes de seguridad para la interoperabilidad entre un enlace UWB y un interferente WiMAX. Se ha observado como el ancho de banda nominal WiMAX afecta al rendimiento del enlace UWB, de forma que ante mayores anchos de banda nominal WiMAX los valores EVM UWB empeoran. Se ha mostrado que un enlace MB-OFDM UWB TFC1 200 Mbit/s funciona correctamente si el interferente WiMAX 10 MHz BW está situado en unos márgenes de seguridad de $d_{\text {WiMAX }}>8 \mathrm{~m}$. Concretamente, para un 
enlace MB-OFDM UWB TFC1 con $\mathrm{d}_{\mathrm{UWB}}=1 \mathrm{~m}$, se garantiza la comunicación si el interferente WiMAX $10 \mathrm{MHz}$ BW está situado en $\mathrm{d}_{\text {WiMAX }} \geq 5 \mathrm{~m}$. En cambio la configuración MB-OFDM UWB TFC5 se ve afectada de forma muy intensa por la presencia del interferente WiMAX, estableciendo un margen de seguridad con distancias para el interferente mayores de $10 \mathrm{~m}$.

Por otra parte, se ha evaluado el EVM relativo de cada subportadora. Esta evaluación ha aportado datos sobre el comportamiento de las subportadoras MB-OFDM UWB ante la interferencia WiMAX. Se ha observado que la degradación de las subportadoras siempre se sitúa en un mismo rango de subportadoras de los símbolos OFDM recibidos. Los resultados aquí obtenidos corroboran la necesidad de establecer técnicas de mitigación de la interferencia para poder garantizar la comunicación UWB ante la presencia de interferentes WiMAX, como por ejemplo en el caso de la configuración MB-OFDM UWB TFC5.

\subsubsection{Prestaciones enlace MB-OFDM UWB con diferentes máscaras espectrales de potencia en presencia de WiMAX 802.16e}

Tras estudiar el comportamiento de un enlace MB-OFDM UWB basado en ECMA-368, se plantea la evaluación de otros escenarios con interferencias controladas que permitan modificar parámetros estándar como la EIRP del sistema. Un ejemplo de estos escenarios, son aquellos de tipo pico-celular en los cuales se cumple la regulación entre las diferentes tecnologías radio y UWB en el límite de la celda, pudiendo relajar esta normativa en el interior de las mismas. Este tipo de escenarios requieren de la implementación de sistemas de monitorización del espectro en toda el área de la celda, que permitan conocer en tiempo real las interferencias, tanto en el interior de la celda como en el límite de cobertura de la misma, y actuar en su mitigación. Es decir, es necesaria la utilización de técnicas avanzadas de mitigación de la interferencia aplicadas a entornos inalámbricos no solo a un dispositivo, siendo estas aplicaciones del tipo radio cognitiva.

En esta Tesis Doctoral se propone modificar ciertos parámetros del estándar como son la canalización en frecuencia y la máscara de potencia espectral. En concreto, se ha evaluado el comportamiento de un enlace MB-OFDM UWB con variaciones de su EIRP ante la presencia de interferentes como WiMAX mediante el montaje experimental mostrado en la Figura 3.22. En este montaje se ha evaluado un enlace UWB de solo $1 \mathrm{~m}$ y $53.3 \mathrm{Mbit} / \mathrm{s}$. La configuración de las señales UWB y WiMAX generadas se basan en los mismos parámetros presentados en la Tabla 3.2 y Tabla 3.3. Para poder modificar la EIRP de la señal UWB transmitida, se ha implementado un par amplificador y atenuador RF tras el módulo generador UWB, Ampl+att en la Figura 3.22. A su vez, se dispone de un amplificador de bajo ruido en el receptor UWB para 
adecuar la señal recibida al margen dinámico del DSA que evalúa el EVM del enlace UWB. Los dispositivos que conforman los enlaces WiMAX y UWB, los equipos de medida y el generador WiMAX utilizados en este montaje experimental son los mismos que los descritos en la sección anterior.

UWB - Tx ANTENA

UWB - Rx ANTENA

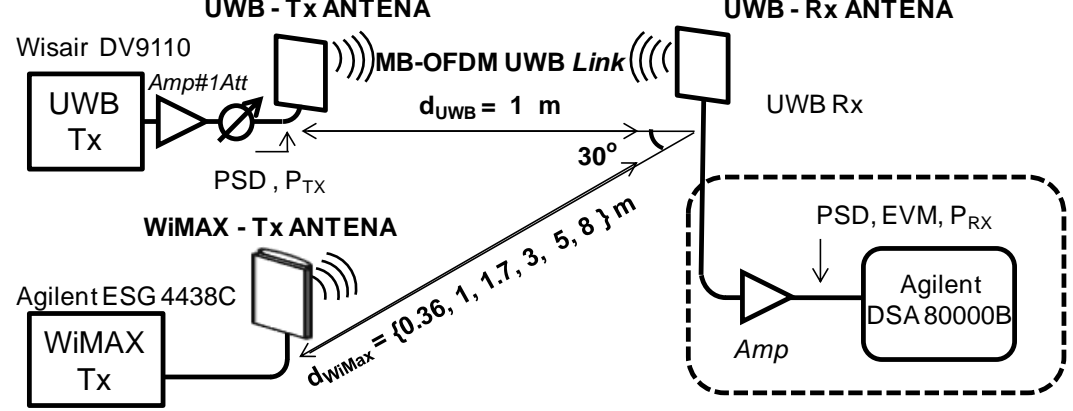

Figura 3.22. Montaje experimental para el estudio del enlace MB-OFDM UWB con variaciones de su EIRP máxima.

Cabe destacar que se han escogido como valores de EIRP el valor normalizado de EIRP máxima $-41.3 \mathrm{dBm} / \mathrm{MHz}$, un valor de $-35 \mathrm{dBm} / \mathrm{MHz}$ y otro valor de $-21 \mathrm{dBm} / \mathrm{MHz}$, todos ellos medidos en la toma de la antena UWB Tx de la Figura 3.22.
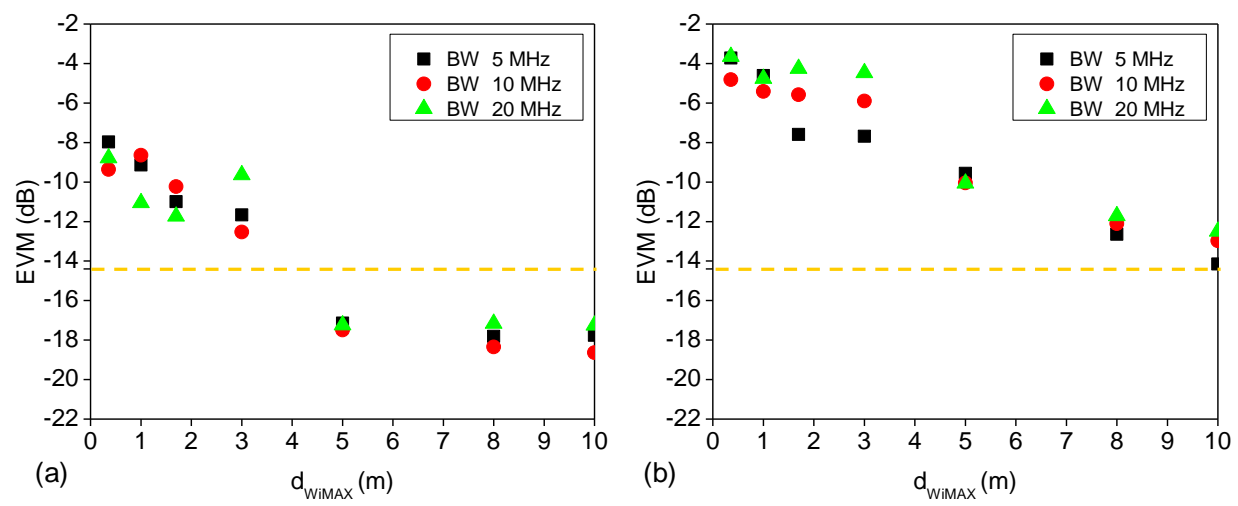

Figura 3.23. EVM medido para un enlace MB-OFDM UWB (a) TFC1 y (b) TFC5 53.3 Mbit/s en presencia de WiMAX, con EIRP según [ECMA368].

En la Figura 3.23 se muestra el EVM medido para el enlace UWB cuando este transmite con una potencia EIRP de $=41.3 \mathrm{dBm} / \mathrm{MHz}$ conforme al estándar. Se observa que para el enlace MB-OFDM UWB TFC1 $53.3 \mathrm{Mbit} / \mathrm{s}$ con $\mathrm{d}_{\mathrm{UWB}}=1 \mathrm{~m}$ los valores se sitúan por debajo del umbral de -14.4 dB EVM UWB cuando el interferente WiMAX está situado en un área de $\mathrm{d}_{\mathrm{WiMAX}} \geq 5 \mathrm{~m}$. A su vez, se observa también que la 
configuración MB-OFDM UWB TFC5 presenta una mayor degradación ante la presencia del interferente WiMAX.
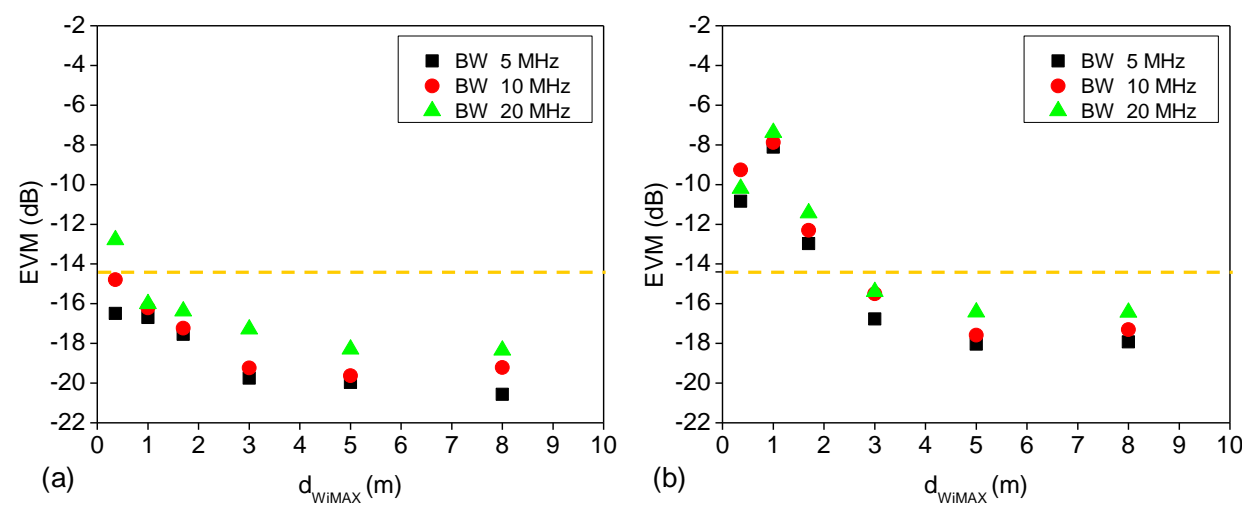

Figura 3.24. EVM medido para enlace MB-OFDM UWB (a) TFC1 y (b) TFC5 53.3 Mbit/s en presencia de WiMAX, con EIRP máxima de $-35 \mathrm{dBm} / \mathrm{MHz}$

En la Figura 3.24 se muestra el EVM medido para un enlace MB-OFDM UWB con una EIRP de transmisión de $-35 \mathrm{dBm} / \mathrm{MHz}$. Se observa que al aumentar el ancho de banda nominal del interferente WiMAX empeoran los valores EVM UWB de este enlace. Sin embargo, la configuración MB-OFDM UWB TFC1 53.3 Mbit/s garantiza la comunicación si el interferente WiMAX está situado a $\mathrm{d}_{\text {WiMAX }} \geq 0.36 \mathrm{~m}$, lo que supone garantizar la comunicación UWB para la mayoría de las distancias del enlace implementado. A su vez, la configuración MB-OFDM UWB TFC5 presenta un comportamiento más robusto ante el interferente WiMAX, puesto que posibilita una comunicación UWB cuando el interferente WiMAX se sitúa a distancias $\mathrm{d}_{\text {WiMAX }} \geq 3 \mathrm{~m}$. Estos valores muestran una mejora notable en el rendimiento del enlace UWB, aumentando de forma significativa su cobertura y la robustez frente a interferencias WiMAX. 

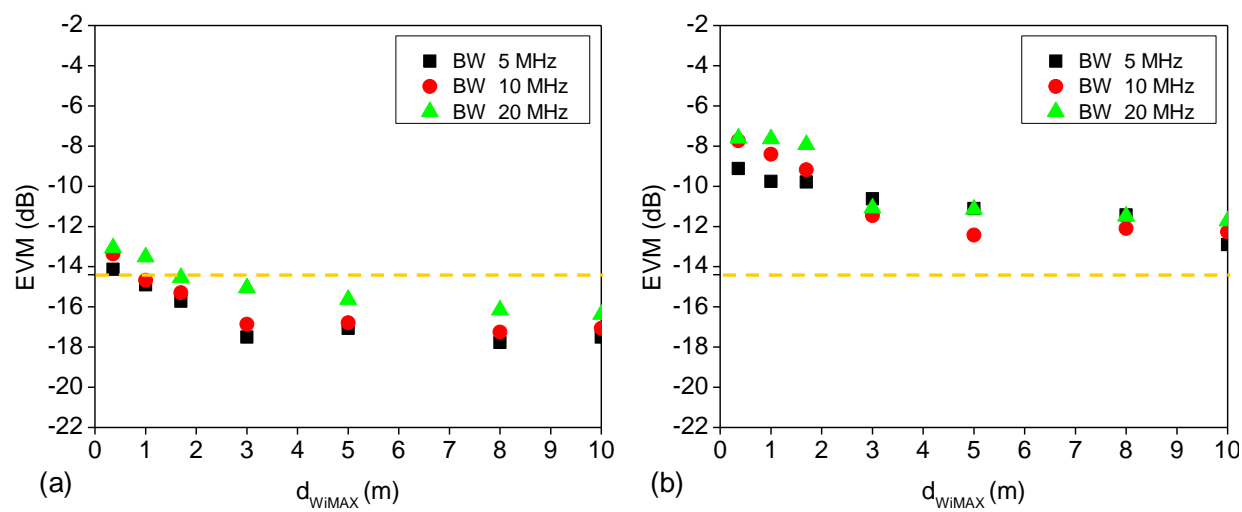

Figura 3.25. EVM medido para un enlace MB-OFDM UWB (a) TFC1, (b) TFC5 53.3 Mbit/s en presencia de WiMAX, con EIRP máxima de $-21 \mathrm{dBm} / \mathrm{MHz}$.

Por último, se evalúa el enlace MB-OFDM UWB con una EIRP máxima en transmisión de $-21 \mathrm{dBm} / \mathrm{MHz}$. En la Figura 3.25 se muestran el EVM obtenido en esta configuración. Para el caso de un enlace MB-OFDM UWB TFC1 en presencia de un interferente WiMAX, la comunicación UWB es viable si el interferente está situado en $\mathrm{d}_{\text {WiMAX }} \geq 1.7 \mathrm{~m}$. En cambio, para la configuración MB-OFDM UWB TFC5 no es posible garantizar la comunicación.

Comparando la Figura 3.24 y la Figura 3.25, se observa como el EVM UWB medido para el enlace MB-OFDM UWB con EIRP de $-21 \mathrm{dBm} / \mathrm{MHz}$ es peor que para $-35 \mathrm{dBm} / \mathrm{MHz}$. Esta situación es debida a cuestiones de saturación de potencia recibida en el receptor UWB, de distorsión de la señal transmitida y de ruido de amplificación en el transmisor UWB debido a los valores elevados de amplificación.

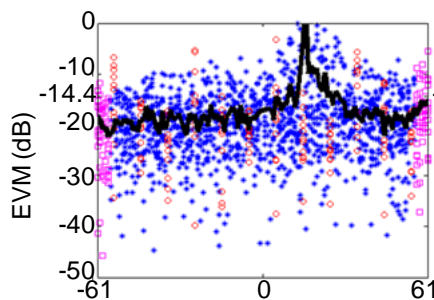

(a) Número Subportadoras

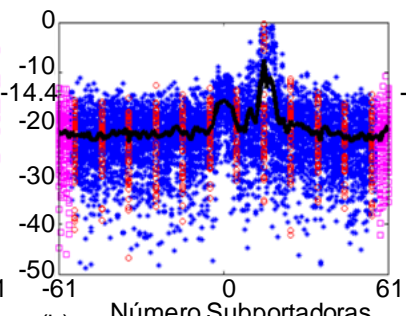

(b)

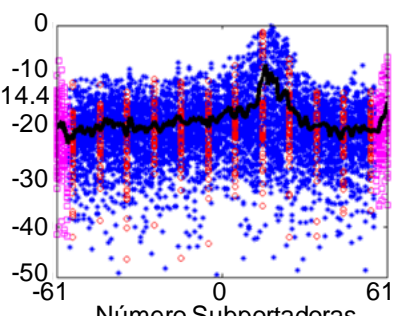

(c)

Figura 3.26. EVM medido por subportadora MB-OFDM UWB TFC1 53.3 Mbit/s con EIRP (a) $-41.3 \mathrm{dBm} / \mathrm{MHz}$ (b) $-35 \mathrm{dBm} / \mathrm{MHz}$ y (c) $-21 \mathrm{dBm} / \mathrm{MHz}$, ante presencia interferente WiMAX 5 MHz BW.

En la Figura 3.26 se muestra el EVM medido para cada subportadora para los enlaces UWB configurados con diferentes valores EIRP. Se observa como el aumento de potencia de transmisión se refleja en una menor degradación del grupo de 
subportadoras afectadas por la interferencia WiMAX. La comparación entre los enlaces de $-35 \mathrm{dBm} / \mathrm{MHz}$ y $-21 \mathrm{dBm} / \mathrm{MHz}$ indica que no se mejoran los valores de EVM, y se observa cómo este aumento empeora el conjunto de valores EVM de todas las subportadoras del sistema. Este comportamiento indica que se introduce una distorsión en el enlace fruto de la amplificación en transmisión o de la saturación del receptor.

Los resultados experimentales mostrados en esta sección indican que el aumento de EIRP no siempre implica una mejora de las prestaciones del enlace UWB y su comportamiento ante interferentes WiMAX. Sin embargo, se ha observado que con -35 dBm/MHz EIRP se aumenta la coexistencia entre UWB y WiMAX comparado con los valores obtenidos para $-41 \mathrm{dBm} / \mathrm{MHz}$. En la Tabla 3.6 se muestran los márgenes de seguridad para la interoperabilidad entre UWB y WiMAX con estas configuraciones UWB.

TABLA 3.6. MÁRgENES INTERFERENTE WiMAX 802.16E PARA COMUNICACIÓN MB-OFDM UWB

\begin{tabular}{|c|c|c|c|}
\hline \multirow{2}{*}{ MB-OFDM UWB } & \multicolumn{3}{|c|}{ EIRP máxima transmitida } \\
\hline & $-41.3 \mathrm{dBm} / \mathrm{MHz}$ & $-35 \mathrm{dBm} / \mathrm{MHz}$ & $-21 \mathrm{dBm} / \mathrm{MHz}$ \\
\hline TFC1 & $\mathrm{d}_{\text {WiMAX }} \geq 5 \mathrm{~m}$ & $\mathrm{~d}_{\text {WiMAX }} \geq 0.36 \mathrm{~m}$ & $\mathrm{~d}_{\text {WiMAX }} \geq 3 \mathrm{~m}$ \\
\hline TFC5 & $\mathrm{d}_{\text {WiMAX }}>10 \mathrm{~m}$ & $\mathrm{~d}_{\text {WiMAX }} \geq 3 \mathrm{~m}$ & $\mathrm{~d}_{\text {WiMAX }}>10 \mathrm{~m}$ \\
\hline
\end{tabular}

En resumen, en este capítulo se ha presentado un estudio experimental de la coexistencia de la tecnología MB-OFDM UWB frente a la tecnología WiMAX Mobile en escenarios de redes personales.

La evaluación del impacto de MB-OFDM UWB sobre un enlace WiMAX 802.16e indica que la robustez del enlace WiMAX está relacionada tanto con el ancho de banda nominal del mismo, como con la distancia en la cual está situado el interferente UWB. En los resultados se observa como al aumentar el ancho de banda nominal en un enlace WiMAX mejora el valor EVM, con lo cual aumenta la calidad del enlace. No obstante, todas las configuraciones WiMAX evaluadas proporcionan resultados por debajo del umbral de - 15 dB EVM WiMAX que garantiza una comunicación WiMAX QPSK 1/2. Sin embargo, la configuración WiMAX $5 \mathrm{MHz}$ BW obtiene valores EVM cercanos al umbral. Esto indica que es conveniente que el interferente UWB esté situado a una distancia superior a $2.5 \mathrm{~m}$ del enlace WiMAX.

El impacto de WiMAX sobre MB-OFDM UWB muestra que el aumento del ancho de banda nominal de WiMAX empeora el EVM UWB medido. Los valores medidos indican que la configuración MB-OFDM UWB TFC5, sin salto en frecuencia, sufre una 
degradación muy intensa debido a la presencia del interferente WiMAX en un área menor a $10 \mathrm{~m}$. Este estudio muestra la necesidad de establecer márgenes de seguridad en torno a los $5 \mathrm{~m}$ para garantizar la interoperabilidad del enlace MB-OFDM UWB ante la presencia de un enlace WiMAX. La evaluación del EVM en MB-OFDM UWB en cada subportadora indica que la interferencia WiMAX siempre afecta a un mismo grupo de subportadoras. El conocimiento del número de subportadoras afectadas y su posición supone una información muy valiosa para desarrollar técnicas de mitigación de la interferencia DAA en futuros desarrollos de dispositivos UWB.

Finalmente, se ha evaluado el comportamiento de enlaces MB-OFDM UWB con una densidad de potencia transmitida EIRP superior a la permitida por el estándar. Los resultados aquí mostrados indican que el aumento de EIRP no siempre implica una mejora la calidad del enlace y su robustez ante el interferente WiMAX, puesto que este aumento de potencia puede conllevar problemas de saturación y distorsión en los transceptores UWB. Sin embargo, se han observado mejoras en los márgenes de protección frente a interferentes WiMAX para una EIRP UWB de $-35 \mathrm{dBm} / \mathrm{MHz}$. Por ejemplo, las distancias de seguridad entre un enlace MB-OFDM UWB TFC1 y un enlace WiMAX $10 \mathrm{MHz}$ BW disminuyen de $5 \mathrm{~m}$ a $0.36 \mathrm{~m}$ al aumentar la EIRP UWB de $-41.3 \mathrm{dBm} / \mathrm{MHz}$ a $-35 \mathrm{dBm} / \mathrm{MHz}$.

Como conclusión, los resultados aquí obtenidos corroboran la necesidad de establecer técnicas avanzadas de mitigación de la interferencia, como DAA, para poder garantizar la comunicación MB-OFDM UWB ante la presencia de interferentes WiMAX, como por ejemplo en el caso de la configuración MB-OFDM UWB TFC5. 


\section{Capítulo 4. Coexistencia e integración en redes de acceso basadas en radio-sobre-fibra}

La distribución conjunta mediante redes ópticas de acceso de señales inalámbricas de elevado régimen binario, surge como respuesta al aumento en la demanda de servicios por parte del usuario como el acceso a Internet de alta velocidad, la demanda de contenidos en línea de alta definición y videojuegos en línea. Esta distribución conlleva cuestiones relacionas con la transmisión de señales inalámbricas en redes ópticas de acceso y su posterior coexistencia en el enlace vía radiofrecuencia hacia el usuario final. Las redes que contemplan la distribución en fibra óptica de señales RF y su posterior acceso radio son denominadas redes de acceso integradas. A su vez, si la transmisión óptica de las señales RF se realiza en su formato final, en base a las frecuencias recogidas por la regulación pertinente, estas redes se denominan sistemas radio-sobre-fibra (RoF, Radio-over-Fibre). Las redes o sistemas RoF constituyen una posibilidad a considerar dentro del concepto de redes de nueva generación (NGN, Next Generation Networks) [Kaz07].

En este capítulo de la Tesis Doctoral, se estudia las prestaciones de la transmisión RoF en redes de acceso de señales UWB y WiMAX. En primer lugar, se exponen los conceptos básicos de la distribución de señales RF en sistemas RoF. Posteriormente, se evalúa la distribución simultánea de señales UWB y WiMAX en sistemas RoF. Finalmente, se propone y demuestra experimentalmente una técnica novedosa de transmisión óptica basada en división por polarización para la distribución conjunta de UWB y WiMAX en sistemas RoF. En definitiva, en este capítulo se presentan los resultados obtenidos de la evaluación de la coexistencia inalámbrica de UWB y WiMAX en sistemas RoF, que permiten delimitar las prestaciones y las limitaciones de su aplicación en el despliegue de redes de acceso integradas. 


\subsection{Componentes básicos en un sistema radio-sobre-fibra}

Los componentes básicos en todo enlace óptico son aquellos que constituyen sus etapas de generación o conversión $\mathrm{E} / \mathrm{O}$, transmisión óptica y recepción o conversión O/E. En la etapa de generación, las señales de RF se transforman a la banda de frecuencias ópticas. En la etapa de transmisión, estas señales se propagan mediante un medio de fibra óptica monomodo o multimodo y finalmente, en recepción, se sitúan dispositivos capaces de recuperar la señal de RF a partir de la señal recibida en frecuencias, como se observa en la Figura 4.1.

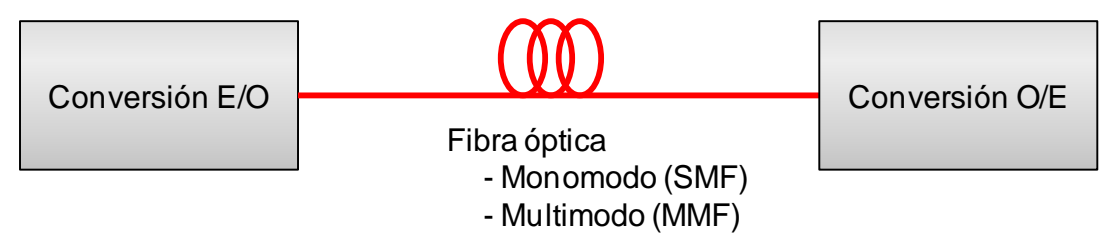

Figura 4.1. Componentes básicos de un sistema RoF.

\subsubsection{Medio óptico}

Las redes de acceso actuales basadas en sistemas RoF se dividen en dos tipos de sistemas según utilicen como medio de transmisión la fibra óptica multimodo (MMF) o la fibra óptica monomodo (SMF), es decir, sistemas radio-sobre-fibra multimodo (RoMMF, Radio-over-Multi-Mode Fibre) y sistemas radio-sobre-fibra monomodo (RoSMF, Radio-over-Single-Mode Fibre). Como ya se comentó en el capítulo 2 de esta Tesis Doctoral, las redes ópticas basadas en fibra MMF, en particular las redes basadas en sistemas RoMMF, han sido ampliamente instaladas en el despliegue de redes de fibra óptica en entornos LAN desde los años 80. Esta amplia implementación es debida a que el mayor diámetro de núcleo de la fibra MMF facilita el conexionado entre fibras [Koo08]. En cambio, los sistemas RoF basados en SSMF se utilizan para la distribución de servicios inalámbricos en last mile, proporcionando servicio a antenas fijas que distribuyen la señal de forma inalámbrica al usuario final, como sucede con las tecnologías LMDS, WiMAX 802.16d o la interconexión de estaciones base de telefonía móvil [Pol99b].

La fibra SMF de tipo estándar tiene un diámetro de núcleo de $9 \mu \mathrm{m}$. Esta fibra es la más implementada en comunicaciones ópticas de larga distancia y su ancho de banda permite capacidades de hasta $41 \mathrm{Pbit} / \mathrm{s}$ por kilómetro [Cha08a].

La fibra MMF más comúnmente instalada tiene un diámetro de núcleo de $62.5 \mu \mathrm{m}$. Dentro de las fibras MMF, cabe destacar la presencia de las denominadas fibras de plástico (POF, Plastic Optical Fibre), con diámetros de $1 \mathrm{~mm}$ de núcleo, lo que implica 
un débil confinamiento de los modos propagados en la fibra y por lo tanto mayores pérdidas que las fibras MMF comunes. Sin embargo, las fibras POF tienen un coste de instalación y mantenimiento muy reducido. La fibra MMF se ha implementado en comunicaciones ópticas de corta y media distancia en entornos interiores como redes universitarias, edificios de oficinas y entornos domésticos. Las redes ópticas basadas en MMF son consideradas de bajo coste, debido a su diámetro y facilidad de manejo.

La fibra MMF se encuentra en redes ópticas de edificios de oficinas con longitudes preinstaladas del orden de $300 \mathrm{~m}$, distancia que permite cubrir la mayoría de conexiones con regímenes binarios de $10 \mathrm{Gbit} / \mathrm{s}$, cumpliendo así con el estándar 10GBASE-SX [Mon08]. Las redes MMF han ido variando históricamente sus características para poder adaptarse a los regímenes binarios que exigen los nuevos estándares para redes locales. La fibra MMF se puede clasificar según el estándar ISO 11801 [ISO11801], basándose en su ancho de banda modal $(\mathrm{MHz} \cdot \mathrm{km})$ y diámetro del núcleo. Según este estándar existen fibras MMF de tipo OM1, que poseen un diámetro de núcleo de $62.5 \mu \mathrm{m}$ con ancho de banda modal de $160-200 \mathrm{MHz} \cdot \mathrm{km}$, las cuales conforman las primeras instalaciones MMF. Posteriormente, al progresar las técnicas de fabricación de fibras ópticas, se consiguieron fibras MMF de menor diámetro como las MMF de tipo OM2, con $50 \mu \mathrm{m}$ de diámetro de núcleo y hasta $500 \mathrm{MHz} \cdot \mathrm{km}$ de ancho de banda modal. Estos dos tipos de fibras son las que actualmente se encuentran preinstaladas mayoritariamente, como indica el informe [Fla07]. Por último, la fibra MMF de tipo OM3 es aquella con $50 \mu \mathrm{m}$ de diámetro de núcleo y hasta $2000 \mathrm{MHz} \cdot \mathrm{km}$ de ancho de banda modal en $850 \mathrm{~nm}$ y optimizada para su uso con VCSEL. Estas fibras MMF tipo OM3 permiten la implementación de $10 \mathrm{GbE}$ en enlaces de hasta $550 \mathrm{~m} \mathrm{MMF}$. El principal problema de las redes MMF es la existencia de distintos tipos de fibra MMF en una misma red, es decir, OM1, OM2 o OM3, puesto que tradicionalmente la actualización de la fibra se realiza por tramos en función de las necesidad.

Las limitaciones asociadas al uso de fibra óptica como medio de transmisión en enlaces RoF son aquellas conocidas en la literatura para enlaces ópticos analógicos. Entre estas limitaciones cabe destacar la dispersión por velocidad de grupo (GVD, Group Velocity Dispersion) también conocida como dispersión cromática, la dispersión por modo de polarización (PMD, Polarization Mode Dispersion) y la dispersión modal asociada a la fibra óptica.

La GVD es consecuencia de la dependencia del índice de refracción de la fibra con la longitud de onda de la señal transmitida. Esta dependencia provoca que las diferentes componentes espectrales de la señal óptica se propaguen a diferente velocidad, ensanchando así la señal y aumentando la posibilidad de interferencia entre símbolos (ISI, Inter-Symbol Interference) en las transmisiones digitales y de atenuación selectiva en frecuencia en las transmisiones tipo RoF [Sch95]. El valor típico de GVD en una 
fibra SSMF para $1550 \mathrm{~nm}$ es de $16 \mathrm{ps} \cdot \mathrm{nm}^{-1} \cdot \mathrm{km}^{-1}$. Se han propuesto métodos de compensación de la GVD mediante el uso de fibras con GVD negativo, como son las fibras SMF compensadoras de la dispersión (DCF, Dispersion-Compensating Fibre). Otros métodos de compensación de la GVD se basan en técnicas de procesado digital, donde se procesa la GVD en el dominio eléctrico de la señal mediante el uso de compensadores y ecualizadores electrónicos [Jan07b].

La PMD es un proceso que tiene su origen en la birrefringencia del núcleo de la fibra óptica, y se ve afectado por factores mecánicos, como las vibraciones o tensiones en la fibra, y por factores ambientales como la temperatura. Para minimizar este proceso, se han propuesto históricamente métodos de fabricación de fibra óptica buscando una menor asimetría en la fabricación del núcleo. Actualmente, los niveles de PMD en fibra óptica son del orden de $0.1 \mathrm{ps} \cdot \mathrm{km}^{-1 / 2}$. A su vez, se ha propuesto la compensación electrónica de la PMD para aplicaciones de distribución de enlaces de señales de microondas, como WiMAX en sistemas RoF [Djo07].

La dispersión modal es un efecto propio de las fibras MMF. La distorsión de la señal óptica por dispersión modal es consecuencia de la diferente velocidad de propagación de cada uno de los modos excitados en la fibra MMF, lo cual afecta de forma notable al ancho de banda de trabajo de la fibra. En estos casos, se ha propuesto la compensación electrónica de la dispersión modal para comunicaciones OFDM y aplicable en redes basadas en sistemas RoMMF según [Loew5].

\subsubsection{Conversión electro-óptica}

La conversión electro-óptica $(\mathrm{E} / \mathrm{O})$ se realiza mediante modulación directa sobre un láser de la señal de RF y modulación indirecta mediante moduladores externos alimentados por fuentes ópticas.

En la conversión E/O mediante modulación externa de señales RF, se utilizan habitualmente moduladores externos de tipo Mach-Zehnder o de electro-absorción [Dag99]. Los moduladores externos poseen un gran ancho de banda, un bajo nivel de señal de control de modulación (drive) y una buena linealidad. Sin embargo, en la mayoría de los casos, los moduladores externos tienen un elevado coste de fabricación debido a su rango de frecuencias de trabajo del orden de milimétricas.

Por el contrario, la conversión mediante modulación directa se considera de bajo coste, ya que se utiliza un solo dispositivo donde la señal moduladora cambia directamente la intensidad del láser. Los principales parámetros de estas fuentes son el ancho de banda de modulación, la longitud de onda óptica y la eficiencia del láser. Con respecto al ancho de banda de modulación, es posible encontrar dispositivos de 
modulación directa como láseres de semiconductor con anchos de banda de hasta $30 \mathrm{GHz}$ [Mat97].

Actualmente, el dispositivo más utilizado para la modulación directa es el diodo láser integrado de semiconductor en una configuración Fabry-Perot, DFB o VCSEL [Cap07]. El laser de semiconductor con estructura Fabry-Perot se forma al introducir dos espejos en una estructura de semiconductor, el cual forma una cavidad donde resuenan modos longitudinales del material, generalmente uniones p-i-n. A su vez, la cavidad tiene un número de modos longitudinales en los cuales se produce una resonancia gracias a una interferencia de tipo constructiva. Por ello, cuando una longitud de onda se encuentra cercana a alguno de los modos longitudinales la cavidad FP se sintoniza a esa longitud de onda para su emisión láser [Agr02]. El láser DFB tiene una estructura similar al Fabry-Perot [Agr02], pero aumenta la selectividad de frecuencias RF en su cavidad mediante el uso de un filtro. En este caso, el filtro utilizado es del tipo ranurado de Bragg (FBG, Fibre Bragg Grating), construido en el medio activo del láser DFB que permite una variación periódica del índice modal del mismo. Existen dispositivos DFB comerciales con anchos de banda funcionales cercanos a $10 \mathrm{GHz}$, y de forma experimental, se ha conseguido hasta $37 \mathrm{GHz}$ de ancho de banda en frecuencias ópticas de $1550 \mathrm{~nm}$ [Bac03]. Por otra parte, en sistemas RoF de bajo coste, se utiliza la tecnología láser de modulación directa VCSEL [Chi03], [Per06]. Esta tecnología se considera de bajo coste debido a sus reducidas dimensiones, puesto que se utilizan cavidades inferiores a $1 \mu \mathrm{m}$ con buenas eficiencias de acoplo que permiten operar en una sola longitud de onda. En estos momentos, la tecnología VCSEL no permite anchos de banda de trabajo tan amplios como los DFB. Existen dispositivos VCSEL que trabajan en las frecuencias ópticas de $850 \mathrm{~nm}, 1310 \mathrm{~nm}$ y $1550 \mathrm{~nm}$. Podemos encontrar VCSEL comerciales con anchos de banda de hasta $3-4 \mathrm{GHz}$ en $1310 \mathrm{~nm}$ y $1550 \mathrm{~nm}$, y de hasta $7 \mathrm{GHz}$ en $850 \mathrm{~nm}$. Otra característica de los VCSEL es su desviación en longitud de onda por temperatura, lo que requiere utilizar sistemas de estabilización en la mayoría de implementaciones con láseres VCSEL [Har03].

\subsubsection{Conversión opto-electrónica}

La última etapa de un sistema RoF es la conversión opto-electrónica (O/E). En ella se recupera la señal modulada en la portadora óptica mediante un foto-detector que convierte la intensidad óptica a corriente eléctrica. Los parámetros clave de un foto-detector son la eficiencia cuántica, el ancho de banda y la linealidad [Agr02]. Los foto-detectores para aplicaciones RoF requieren un ancho de banda de trabajo suficiente, una alta eficiencia en la conversión $\mathrm{O} / \mathrm{E}$ y soportar niveles altos de potencia óptica. Existen fotodiodos (PD, Photo-Diode) de alta velocidad en las bandas de $1310 \mathrm{~nm}$ y $1550 \mathrm{~nm}$ con anchos de banda de hasta $300 \mathrm{GHz}$ [Ito00], y PD comerciales con anchos de banda de trabajo de hasta $100 \mathrm{GHz}$. Cabe destacar la posibilidad de 
utilizar foto-receptores, es decir, un dispositivo que integra un PD y un amplificador RF. Los foto-receptores permiten aumentar la sensibilidad del PD y mejorar su eficiencia de conversión $\mathrm{O} / \mathrm{E}$, aunque el ancho de banda del conjunto esté limitado por el amplificador integrado.

\subsubsection{Integración del acceso inalámbrico}

En cuanto a la distribución simultánea de múltiples servicios en sistemas RoF, es habitual su implementación en redes de acceso basadas en tipologías inalámbricas pico-celulares [Wak02a], [Sau07]. Las tipologías pico-celulares proporcionan servicios inalámbricos a entornos WPAN o de reducido tamaño. En este caso, los sistemas RoF distribuyen múltiples servicios simultáneamente a las diferentes pico-células WPAN, creando redes distribuidas de pico-celdas que permiten ampliar la cobertura. Un ejemplo de este tipo de sistemas son las redes distribuidas de antenas, que dan servicio mediante la utilización de redes de acceso basadas en distribución RoF.

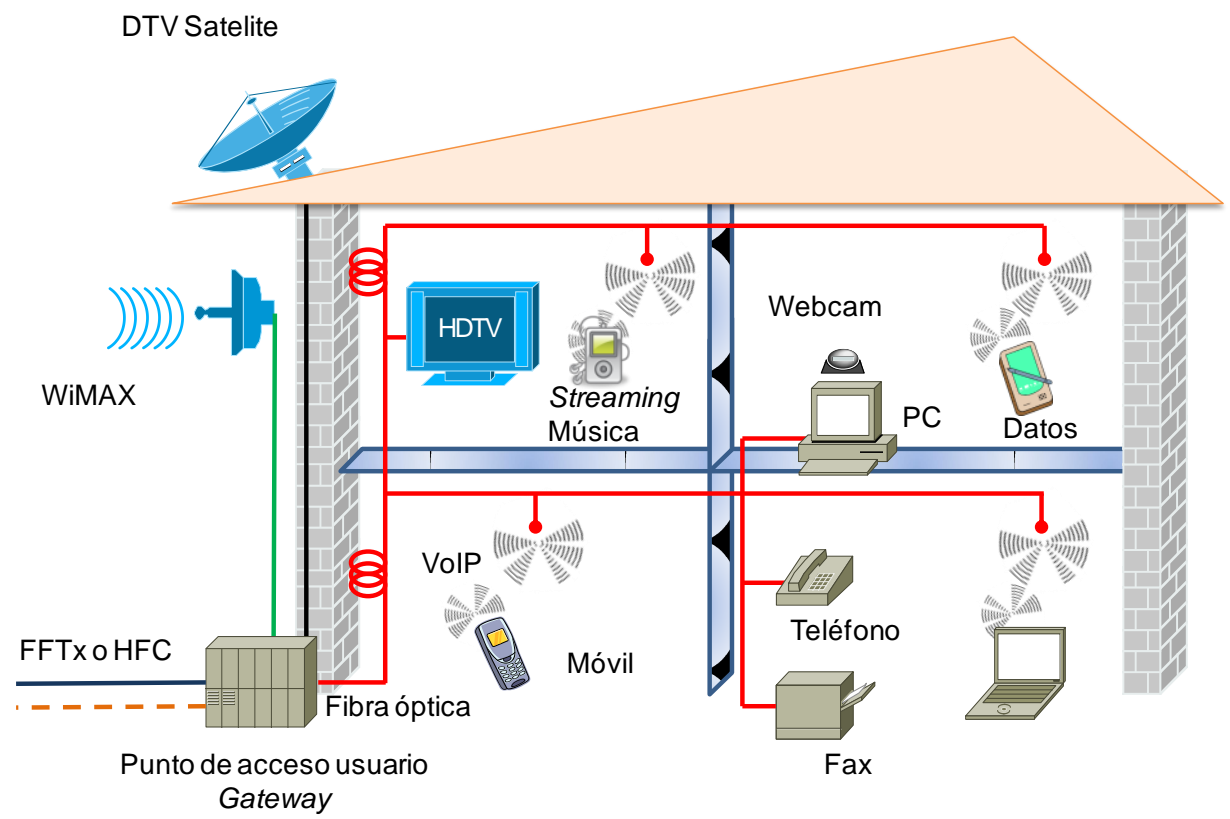

Figura 4.2. Representación de distribución multiservicio en entornos interiores basada en redes con transmisión RoF.

En la Figura 4.2 se representa una red basada en RoF que distribuye múltiples servicios de diversos operadores en un entorno de tipo doméstico, similar a las redes distribuidas pico-celulares y a las redes de antenas distribuidas. 
La demanda de acceso a servicios de elevados regímenes binarios proporcionados por tecnologías radio emergentes como UWB y WiMAX, conlleva cambios en las redes de distribución basadas en RoF, puesto que deben dar acceso a mayor número de usuarios con mayores regímenes binarios. Como se comentó en el capítulo 2 de esta Tesis Doctoral, una solución propuesta en [Sau07] es la reducción del área de cobertura radio, lo cual reduce el número de usuarios. En este caso, para dar cobertura al mismo número de usuarios, se debe aumentar el número de pico-células en los sistemas RoF. Otras estrategias para el aumento de la cobertura total del sistema son la utilización de técnicas MIMO o técnicas de beamforming en las antenas [Kob08]. Estas soluciones conllevan el aumento de los elementos E/O, como las antenas, y por lo tanto aumenta la complejidad en la gestión de los mismos.

Uno de los aspectos más importantes en el desarrollo de los sistemas RoF como base de redes de acceso, es asegurar la convergencia inalámbrica para la distribución de múltiples servicios dentro de la misma celda de cobertura. La convergencia inalámbrica conlleva la coexistencia en la distribución óptica y posterior acceso radio integrado entre diferentes señales radio, por ejemplo señales WiMAX para el acceso en entornos WWAN y transmisiones UWB para comunicaciones WPAN.

Actualmente, la mayor parte de la fibra óptica instalada en redes de edificios y universidades o in-building, entre un $85 \%$ y un $90 \%$, es fibra multimodo [Fla07]. Por este motivo, uno de los campos de mayor interés es la investigación de técnicas de transmisión sobre MMF que permitan la reutilización de la infraestructura existente para la distribución de múltiples servicios inalámbricos. Los sistemas RoMMF se han utilizado para la distribución de señales radio como IEEE 802.11, GSM o UMTS en entornos de oficinas mediante redes de antenas distribuidas [Cri09]. A su vez, también se han propuesto sistemas RoMMF que integran la convergencia inalámbrica de múltiples servicios. Por ejemplo, la distribución centralizada de IR-UWB y IEEE 80.11b WLAN con enlaces de fibra MMF de hasta $600 \mathrm{~m}$ propuesta en [Luo05]. Otro ejemplo, lo tenemos en la evaluación de un sistema pico-celular híbrido RoMMF para la transmisión simultánea de Gigabit Ethernet, WLAN IEEE 802.11a/g e IR-UWB propuesta en [Sim05], que permite enlaces de fibra MMF de hasta $300 \mathrm{~m}$.

En cuanto a la distribución simultánea de señales en sistemas RoF con fibra SSMF, se han propuesto diversos sistemas RoSSMF para la distribución simultánea de señales WLAN y UWB. Por ejemplo, en [Guo08], se ha propuesto la distribución simultánea de MB-OFDM UWB y WLAN 802.11a/g para enlaces de fibra SSMF de hasta $5 \mathrm{~km}$. A su vez, la saturación de las frecuencias libres ISM por debajo de $10.66 \mathrm{GHz}$, como son $2.4 \mathrm{GHz}, 3.5 \mathrm{GHz}$ y $5 \mathrm{GHz}$, y la necesidad de reducir el tamaño de las celdas de cobertura inalámbricas, plantea el uso de bandas superiores de frecuencias milimétricas como $40 \mathrm{GHz}$ y $60 \mathrm{GHz}$. En este caso, se han propuesto esquemas de modulación SSB 
para conseguir regímenes binarios de hasta $2.5 \mathrm{Gbit} / \mathrm{s}$ para portadoras de $40 \mathrm{GHz}$ sobre $44 \mathrm{~km}$ de fibra SSMF [Wib05], y de $10 \mathrm{Gbit} / \mathrm{s}$ con enlaces $21 \mathrm{~km}$ de fibra SSMF y $2 \mathrm{~m}$ de alcance radio para portadoras $\mathrm{RF}$ a $33 \mathrm{GHz}$ [Gon09].

En el capítulo 3 de esta Tesis Doctoral, se ha evaluado la coexistencia de UWB y WiMAX en entornos WPAN. En este capítulo, se evaluará la coexistencia e integración de UWB y WiMAX en redes basadas en sistemas RoF multimodo y monomodo. Los resultados obtenidos permitirán evaluar y definir las prestaciones de una red de acceso basada en RoF con convergencia inalámbrica para servicios de elevado régimen binario

\subsection{Análisis experimental de la coexistencia UWB y WiMAX en sistemas radio-sobre-fibra multimodo}

Tras analizar los componentes básicos de los sistemas RoF se propone la transmisión conjunta de UWB y WiMAX en sistemas RoF utilizando fibra multimodo (RoMMF) como medio óptico de transmisión. En este apartado se evalúan experimentalmente las prestaciones de la técnica propuesta y se identifican los principales factores que limitan la transmisión conjunta UWB y WiMAX en sistemas RoMMF.

\subsubsection{Transmisión en fibra óptica multimodo}

Las prestaciones de los sistemas RoMMF para la distribución conjunta de señales inalámbricas con elevados regímenes binarios están limitadas por la dispersión modal asociada a las fibras MMF, que reduce su ancho de banda efectivo. Esta limitación es importante teniendo en cuenta que se disponen de fibras de tipo OM1 y OM2 preinstaladas en la mayoría de redes de distribución interiores. Para el desarrollo de sistemas RoMMF, se han propuesto diversas técnicas de transmisión sobre MMF que intentan aumentar este ancho de banda, soslayando la limitación impuesta por la dispersión intermodal asociada a la fibra MMF [Koo08]. Entre estas técnicas, se encuentra la multiplexación por diversidad de grupos de modos, en la cual la excitación selectiva de subconjuntos de modos en la fibra MMF produce canales paralelos de comunicación [Stu00], [Koo02], [Koo03]. Otra técnica es la multiplicación de frecuencia óptica (OFM, Optical Frequency Multiplication), que se basa en la generación de armónicos de RF mediante conversión de modulación en frecuencia en modulación de intensidad (FM-IM), gracias a un filtro óptico paso banda periódico [Lar06]. Otras técnicas emplean la codificación multinivel, como CDMA [Rad97], o técnicas MIMO [Sha05]. Sin embargo, la técnica más empleada es la multiplexación por subportadora SCM [Ty102], [Wak02b], así como las técnicas de multiplexación por longitud de onda WDM [Gu06]. La aplicación de estas técnicas de transmisión óptica sobre MMF posibilita la realización de sistemas RoMMF con tecnologías radio en 
milimétricas, facilitando su uso como soporte para las redes de acceso inalámbrico [Yue04], [Pep05].

Una vez expuestas las principales características de los sistemas RoMMF, a continuación se evalúa su funcionamiento ante la distribución simultánea de comunicaciones radio que proporcionan elevados regímenes binarios: WiMAX y UWB.

Como se ha comentado en el capítulo 2 de esta Tesis Doctoral, WiMAX y UWB son tecnologías radio que permiten transmisiones de elevados regímenes binarios, hasta $1 \mathrm{Gbit} / \mathrm{s}$ en UWB y hasta $12 \mathrm{Mbit} / \mathrm{s}$ en WiMAX. UWB es un sistema radio de banda ultra-ancha cuyos canales radio ocupan bandas de más de $500 \mathrm{MHz}$ [FCC02.48]. En cambio, WiMAX es un sistema radio con anchos de banda de hasta $20 \mathrm{MHz}$ [Koh04], [IEEE80216e]. El espectro UWB ocupa la banda de frecuencias de $3.1 \mathrm{GHz}$ a $10.6 \mathrm{GHz}$ para MB-OFDM UWB [ECMA368]. UWB es un sistema sin licencia de explotación cuyo espectro se solapa con otros sistemas radio con licencia, como WiMAX. UWB y WiMAX se solapan en las bandas de frecuencia de $3.3 \mathrm{GHz}$ a $3.8 \mathrm{GHz}$ y de $5.4 \mathrm{GHz}$ a $5.825 \mathrm{GHz}$. UWB permite proveer elevados regímenes binarios en escenarios de corto alcance, del tipo WPAN, como oficinas u hogares. Sin embargo, WiMAX implementa servicios de elevado régimen binario en redes inalámbricas de tamaño medio, como WMAN en campus universitarios y WLAN en centros comerciales o edificios de oficinas [Pra06]. Estas tecnologías son complementarias, como se observa al comparar las velocidades y los escenarios de aplicación de ambas. A su vez, la coexistencia en un mismo espacio físico es una consecuencia lógica del desarrollo de las comunicaciones radio en entornos de tipo oficina o interiores.

En este tipo de entornos in-building o interiores, las redes ópticas basadas en MMF predominan sobre las basadas en SMF. Como se ha comentado al inicio de este capítulo, las redes ópticas basadas en MMF suponen el $90 \%$ de las redes preinstaladas en oficinas y con enlaces de hasta $300 \mathrm{~m}$. Además, las soluciones de bajo coste RoF se basan en enlaces de fibra MMF, debido a su fácil manejo y bajo coste de mantenimiento y despliegue, y en el uso de dispositivos de bajo coste como los láseres VCSEL y los foto-receptores, generalmente operando en $850 \mathrm{~nm}$ [Das06], [Mar02]. Estas características de presencia de infraestructura preinstalada y bajo coste, son las que posicionan las redes basadas en sistemas RoMMF como candidatas para la evaluación de distribución simultánea de WiMAX y UWB.

En la actualidad, la utilización de RoMMF para la distribución de señales UWB permite aumentar la cobertura del enlace radio UWB estándar, por ejemplo, se estima una cobertura estándar de 3-4 metros para MB-OFDM UWB a $200 \mathrm{Mbit} / \mathrm{s}$ [ECMA05]. A su vez, permite desplegar una cobertura en todo el hogar, como muestra la Figura 4.4. En esta figura, se muestran dos ejemplos de la extensión del acceso radio UWB 
mediante RoMMF en edificios, basados en segmentación celular e interconexión de áreas dispersas, respectivamente. Los sistemas RoMMF responden al concepto de distribución mediante RoF de tipo pico-celular, y conllevan la centralización del procesado de las señales y de la gestión de los servicios asociados, permitiendo un rápido despliegue y un menor coste de mantenimiento de los sistemas. Estudios recientes de sistemas MB-OFDM UWB sobre MMF indican la posibilidad de establecer enlaces RoF MB-OFDM UWB de 600 metros MMF para $53.3 \mathrm{Mbit} / \mathrm{s}$ [Yee07], y también enlaces de $750 \mathrm{~m}$ de longitud de fibra MMF y $1 \mathrm{~m}$ de alcance radio [Ben08].

(a)

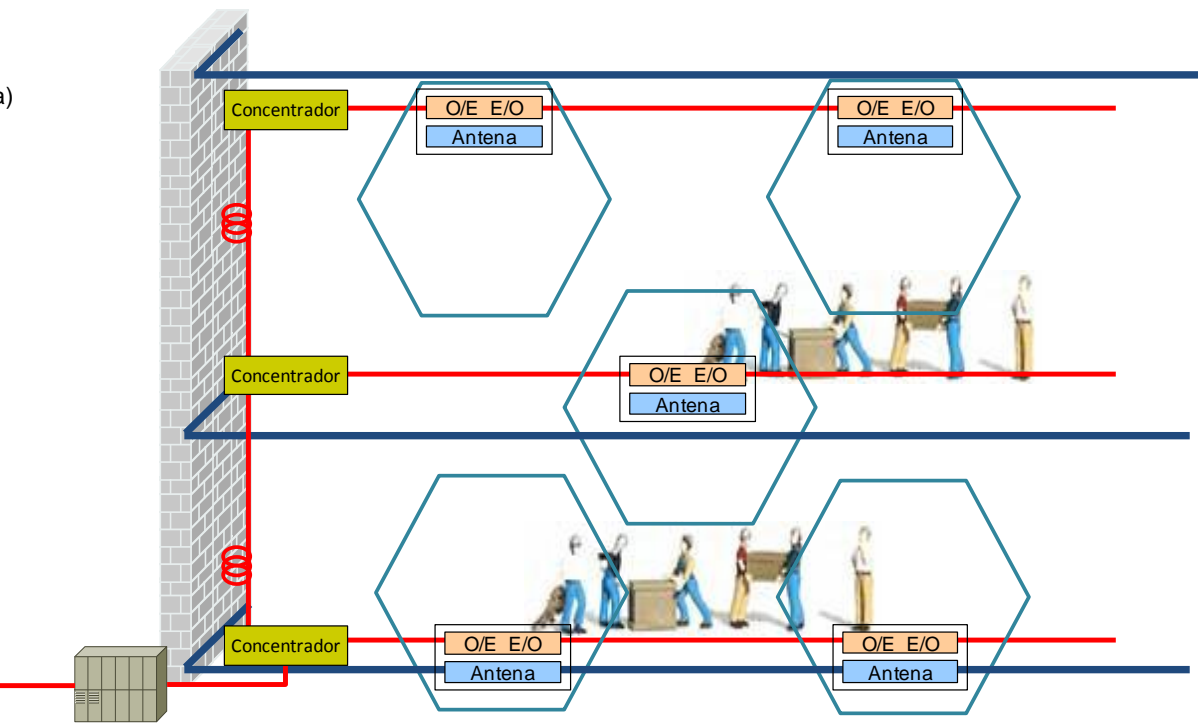

Punto de acceso usuario

Gateway

(b)

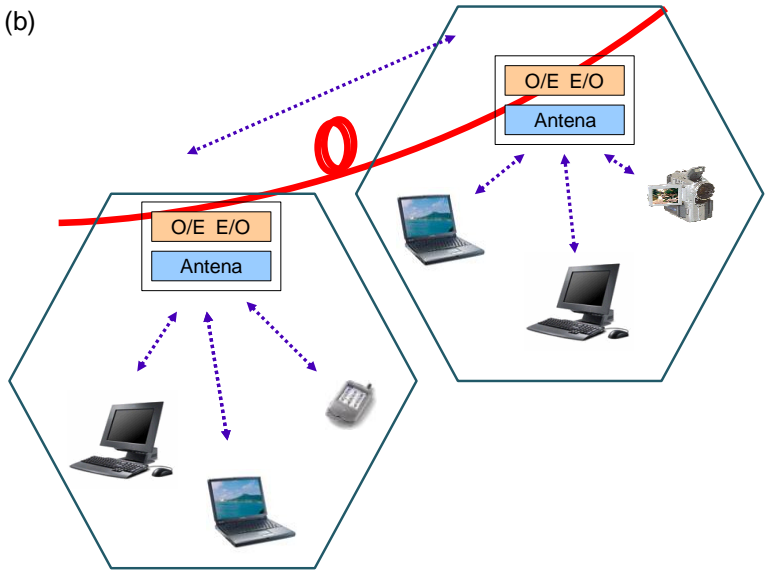

Figura 4.3. Ejemplos de aplicación de sistemas RoMMF en la distribución de UWB para la extensión del acceso mediante (a) la segmentación celular en edificios y (b) la conexión entre áreas dispersas [Ran07]. 
La distribución de señales WiMAX mediante sistemas RoMMF, permite aumentar el área de cobertura utilizando redes de antenas distribuidas (DAS) [Sal87b]. El uso de RoMMF para la distribución de señales WiMAX permite una gran flexibilidad en el diseño de las redes DAS, así como una cobertura cuasi continua para espacios como túneles o áreas comerciales. Ejemplos de la implantación de este tipo de redes han sido la cobertura inalámbrica en eventos deportivos, como la copa del mundo de fútbol del 2006 de Alemania [Cas03].

En la presentación de los resultados experimentales en este capítulo de la Tesis Doctoral se sigue la siguiente notación. WiMAX $10 \mathrm{MHz}$ BW Pol. A, es una señal WiMAX 802.16e configurada con un ancho de banda nominal de $10 \mathrm{MHz}$, donde el valor del ancho de banda nominal varía según el estudio experimental, configurada según una polarización de tipo Pol. A, la cual puede variar según el esquema implementado. MB-OFDM UWB TFC1 $200 \mathrm{Mbit} / \mathrm{s}$, es una señal MB-OFDM UWB configurada con un código TFC de salto en frecuencia y un régimen binario de $200 \mathrm{Mbit} / \mathrm{s}$. Estos parámetros pueden variar según el estudio experimental. Por último, EVM UWB o EVM WiMAX son el EVM medido sobre las señales UWB o WiMAX, respectivamente.

\subsubsection{Montaje experimental}

La coexistencia inalámbrica de UWB y WiMAX en sistemas RoMMF se ha planteado en esta Tesis Doctoral desde el punto de vista de la transmisión en fibra y las limitaciones en la propagación radio. Para ello, se propone la evaluación experimental de la coexistencia entre MB-OFDM UWB y WiMAX 802.16d en una transmisión conjunta sobre un enlace MMF y su posterior transmisión radio. En este estudio se escoge WiMAX 802.16d debido a que el objetivo es la extensión de la cobertura WiMAX en el interior de un edificio. A su vez, se considera la distribución simultánea de datos con elevados regímenes binarios en base a UWB, como señales HDTV.

Este estudio experimental se ha llevado a cabo en un entorno de oficinas de un edificio de investigación de la Universidad Politécnica de Valencia. Este entorno se caracteriza por presentar zonas de visibilidad parcial, es decir, el emisor se ubica en el interior de una sala de reuniones, mientras que el receptor se traslada a lo largo de un pasillo anexo.

En la Figura 4.4 se muestra el esquema del montaje experimental realizado para evaluar la coexistencia inalámbrica de MB-OFDM UWB y WiMAX 802.16d en un sistema RoMMF. 


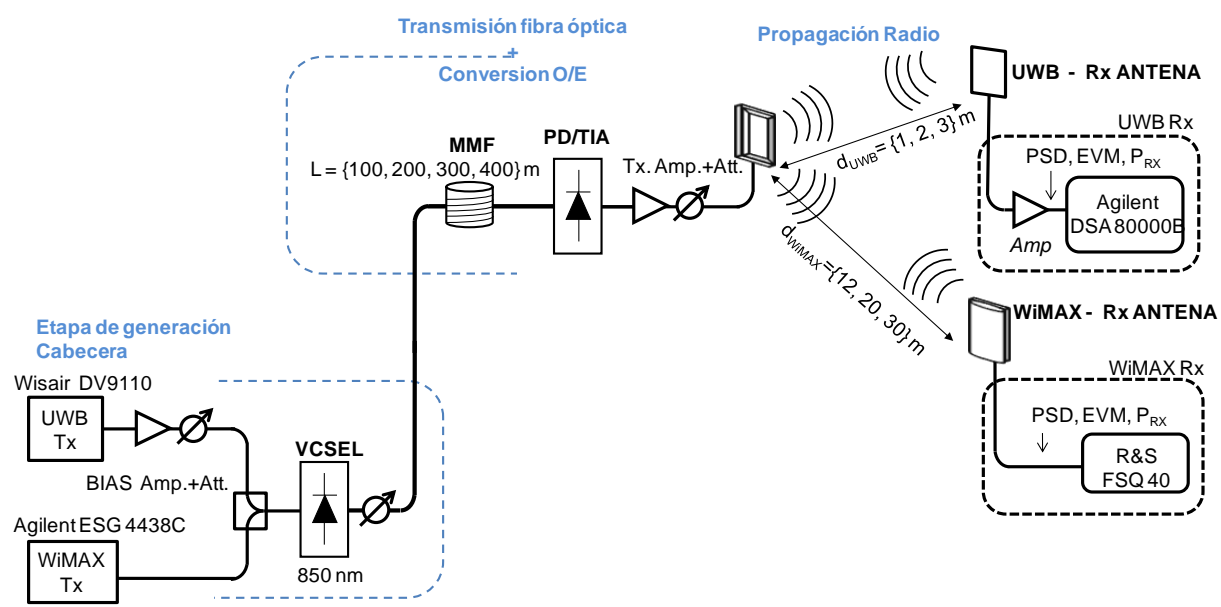

Figura 4.4. Montaje experimental para la evaluación de la coexistencia inalámbrica mediante un sistema RoMMF.

Este montaje experimental se ha dividido en tres secciones principales. La primera sección contempla la etapa de cabecera o generación de señales MB-OFDM UWB y WiMAX IEEE 802.16d. La segunda sección está formada por la etapa de transmisión óptica en MMF y la conversión O/E. La última sección está formada por el canal de propagación radio y los equipos receptores del usuario final.

La generación de las señales UWB y WiMAX se ha realizado en la sección de cabecera. La señal WiMAX se ha implementado de acuerdo al estándar IEEE 802.16d [IEEE80216e] mediante un generador de señales vectoriales, Agilent ESG E4438C, y un software de procesado de señales. Se ha generado una señal WiMAX con una frecuencia central de $3.5 \mathrm{GHz}$ y un ancho de banda de $3.5 \mathrm{MHz}$. La señal WiMAX implementa una modulación 64 QAM, consiguiendo regímenes binarios de $13.1 \mathrm{Mbit} / \mathrm{s}$. Los principales parámetros de la señal WiMAX están resumidos en la Tabla 4.1.

TABla 4.1. Parámetros de la SEÑAL WiMAX IEEE 802.16D geNERADA

\begin{tabular}{l|c}
\hline \hline Frecuencia central & $3.5 \mathrm{GHz}$ \\
\hline Ancho de banda nominal & $3.5 \mathrm{MHz}$ \\
\hline N. Subportadoras & 256 \\
\hline Modulación & $64 \mathrm{QAM}$ \\
\hline Espacio entre subportadoras & $15.625 \mathrm{kHz}$ \\
\hline Régimen binario & $13.1 \mathrm{Mbit} / \mathrm{s}$ \\
\hline \hline
\end{tabular}

La señal MB-OFDM UWB se ha implementado mediante un módulo de comunicaciones UWB de evaluación avanzada, el modelo DV9110 de la compañía Wisair Ltd. Las señales MB-OFDM UWB se han generado conforme al estándar 
ECMA 368 [ECMA368]. Estas señales están centradas en la banda BG1 de MB-OFDM UWB, de $3.1 \mathrm{GHz}$ a $4.75 \mathrm{GHz}$, que comprende tres subbandas de $528 \mathrm{MHz}$ de ancho de banda. En la sección UWB se opta por generar una transmisión QPSK de $200 \mathrm{Mbit} / \mathrm{s}$ con un código de tiempo-frecuencia TFC1 que implica salto en frecuencia entre las tres subbandas, como se comentaba en el capítulo 2 de esta Tesis Doctoral. Los principales parámetros de la señal UWB generada se muestran en la Tabla 4.2.

TABla 4.2. ParÁMETROS de la SEÑAl MB-OFDM UWB GENERAdA

\begin{tabular}{l|c}
\hline \hline TFC & TFC1 \\
\hline \multirow{2}{*}{ Banda de frecuencias } & BG1 \\
& $\{3.168-4.752 \mathrm{GHz}\}$ \\
\hline Frecuencia central & $3.96 \mathrm{GHz}$ \\
\hline Régimen binario & $200 \mathrm{Mbit} / \mathrm{s}$ \\
\hline EIRP & $-41.3 \mathrm{dBm} / \mathrm{MHz}$ \\
\hline \hline
\end{tabular}

Las señales MB-OFDM UWB y WiMAX generadas se modulan de forma directa mediante un láser de tipo VCSEL para su conversión E/O. Para asegurar que el VCSEL se encuentra en la región de trabajo más eficiente y lineal, se ha utilizado un par amplificador RF de bajo ruido (LNA, Low Noise Amplifier) y atenuador RF, denominado BIAS Amp.+Att. en la Figura 4.4. Esto permite ajustar la potencia de la señal UWB y WiMAX entregada al VCSEL. Las señales WiMAX y MB-OFDM UWB se combinan eléctricamente antes de ser convertidas al dominio óptico a través de la modulación directa de un VCSEL que trabaja en la ventana de $850 \mathrm{~nm}$, VCSEL Truelight TTR-1F45-447 4.25 Gbps.

La segunda sección contempla la transmisión mediante fibra MMF de las señales moduladas en el VCSEL para su posterior foto-detección. En este caso, se ha considerado una fibra MMF con un núcleo de diámetro $50 \mu \mathrm{m}$, es decir fibra MMF de tipo OM2. Para esta evaluación, las longitudes de la fibra MMF escogidas son de $100 \mathrm{~m}, 200 \mathrm{~m}, 300 \mathrm{~m}$ y $400 \mathrm{~m}$. La conversión O/E se realiza mediante un foto-detector PIN con amplificador de transimpedancia integrado (TIA, Transimpedance Amplifier) en $850 \mathrm{~nm}$, Truelight TRR-8F55-643 4.25Gbps 850nm GaAs PINTIA. Tras la foto-detección, la señal RF se amplifica mediante un LNA para ajustar cada señal a los parámetros de potencia especificados en cada estándar. Para ello, se emplea un LNA con una ganancia de $40 \mathrm{~dB}$ y una figura ruido de $2.5 \mathrm{~dB}(\mathrm{G}=40 \mathrm{~dB}, \mathrm{NF}=2.5 \mathrm{~dB})$. De esta forma se completa la etapa de transmisión óptica en MMF y la conversión O/E.

La señal eléctrica procedente del LNA alimenta a una antena para su propagación radio hacia los usuarios finales. La antena de transmisión escogida es el modelo HUBER+SUHNER SWA 0764/360/6/30/V. Se trata de una antena de interiores 
multiservicio, diseñada para distribuir múltiples señales inalámbricas como Wi-Fi, WiMAX, Bluetooth y UWB, y con una ganancia de $7.5 \mathrm{dBi}$.

La sección final agrupa la transmisión inalámbrica conjunta hacia el usuario final, contemplando el canal radio y los receptores o usuarios finales UWB y WiMAX. La transmisión inalámbrica simultánea de WiMAX y UWB se ha realizado sobre un enlace radio cuyas longitudes varían según la situación del receptor UWB y WiMAX.

El receptor UWB lo conforma una antena omnidireccional diseñada para comunicaciones UWB de tipo patch, In4Tel-omni con $0 \mathrm{dBi}$ de ganancia, y un analizador de señales digitales con un software de análisis vectorial para evaluar los parámetros de calidad del enlace UWB, Agilent DSA 80000B y software VSA 89600, respectivamente. Cabe destacar la inclusión de un LNA tras la antena receptora UWB, para poder compensar las pérdidas introducidas por el canal radio y adaptar la señal recibida al margen dinámico del DSA, como se observa en el conjunto UWB Rx de la Figura 4.4.

El receptor WiMAX está compuesto por una antena plana de tipo subscriber para comunicaciones WiMAX con $9 \mathrm{dBi}$ de ganancia, HUBER+SUHNER SPA 3500/65/9/0V, y un analizador de espectros digital avanzado, R\&S FSQ40, como se observa en el conjunto WiMAX Rx de la Figura 4.4. El analizador FSQ40 lleva asociado un software para el análisis de comunicaciones WiMAX basadas en IEEE $802.16 \mathrm{~d} / \mathrm{e}$, lo que permite evaluar los parámetros de la calidad del enlace radio WiMAX desplegado.

Para poder comparar la calidad de la transmisión de las señales UWB y WiMAX en este sistema RoMMF, se opta por el estudio de la magnitud de error vectorial recibida (EVM, Error Vector Magnitude), conocida figura de mérito en las comunicaciones digitales.

\subsubsection{Evaluación experimental prestaciones coexistencia inalámbrica}

En primer lugar, se analiza el comportamiento del VCSEL ante la potencia RF entregada por parte de la sección de generación de señales UWB y WiMAX. Este análisis permite estimar el rango óptimo de potencia de señales RF que maximizan la eficiencia de conversión E/O.

En la Figura 4.5 se representa el EVM medido para MB-OFDM UWB y WiMAX 802.16d cuando estas señales se transmiten de forma individual sobre el enlace de fibra MMF. Se presentan los datos obtenidos para enlaces de $100 \mathrm{~m}$ a $400 \mathrm{~m}$ MMF y también para una transmisión sin fibra, directamente del VCSEL al PIN/TIA denominada B2B (Back-to-Back). La potencia de RF media a la entrada del VCSEL de 
cada servicio inalámbrico se ha variado dentro de los márgenes que permiten ambas tecnologías. A su vez, se ha representado mediante una línea discontinua el umbral de EVM que asegura una comunicación viable tanto para MB-OFDM UWB, $-14.4 \mathrm{~dB}$ EVM UWB [ECMA368], como para WiMAX, -31 dB EVM WiMAX para una comunicación WiMAX 64 QAM [IEEE80216d].
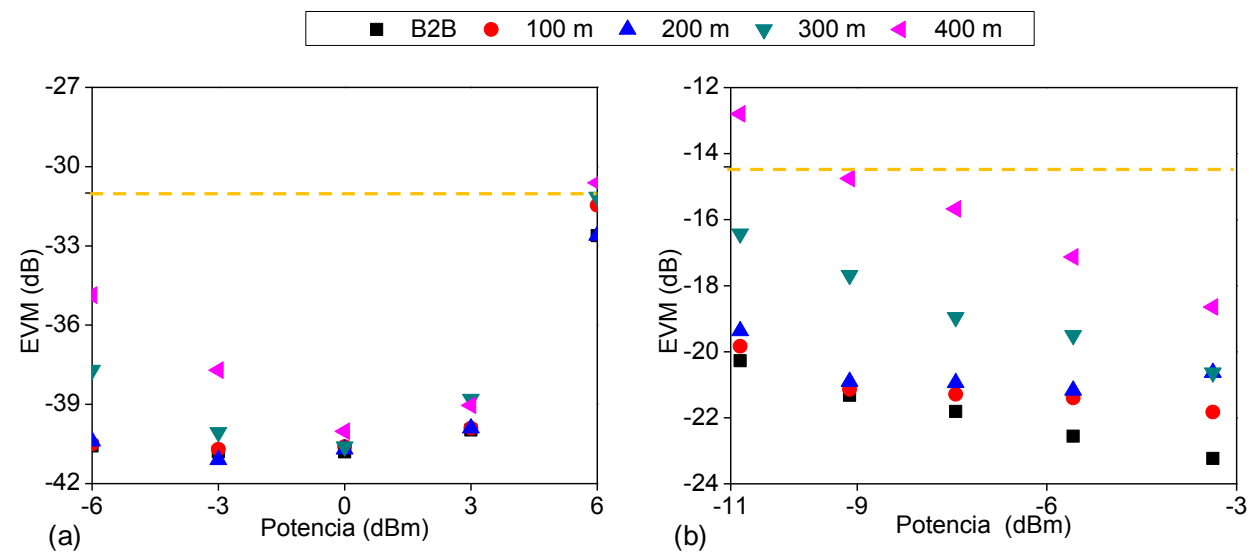

Figura 4.5. EVM medido para diferentes potencias RF en VCSEL tras la transmisión sobre MMF de la señal (a) WiMAX 802.16d y (b) MB-OFDM UWB.

En la Figura 4.5 (a) se observa como para valores bajos de potencia RF WiMAX de entrada, se produce una degradación en el EVM WiMAX del enlace MMF, aunque se mantiene por debajo del umbral de -31 dB EVM WiMAX en todos los casos evaluados. En la Figura 4.5 (b) se observa esta distorsión para valores bajos de potencia RF UWB a la entrada del VCSEL, llegando en este caso a sobrepasar el umbral EVM UWB. Sin embargo, en ambas gráficas se observa el efecto de la dispersión modal asociada a la fibra MMF. Esta dispersión modal se traduce en una degradación lineal conforme aumenta la distancia MMF bajo estudio, lo que provoca que para un enlace de $400 \mathrm{~m}$ MMF los valores de EVM para UWB sufran una degradación más intensa. Para configurar correctamente la cabecera RF, se han escogido valores de RF donde el efecto predominante sea la dispersión modal en MMF y no la distorsión introducida por los valores de la potencia RF de entrada. En base a los valores EVM mostrados en la Figura 4.5 , se escogen valores de potencia RF entre $0 \mathrm{dBm}$ a $3 \mathrm{dBm}$ para WiMAX y de $-6 \mathrm{dBm}$ a $-3 \mathrm{dBm}$ para MB-OFDM UWB.

Por último, se configura el par amplificador atenuador asociado al generador UWB para asegurar la coexistencia RF de ambos sistemas. se selecciona un rechazo entre ambos de unos $35 \mathrm{~dB}$ [ECC120]. Para ello, se escoge un valor de $3 \mathrm{dBm}$ de potencia $\mathrm{RF}$ en la banda WiMAX y de $-6 \mathrm{dBm}$ de potencia en la banda utilizada por MB-OFDM UWB. 
En la Figura 4.6 (a) se muestra el espectro RF conjunto tras la adecuación de los niveles de la cabecera de RF a la entrada del VCSEL. En la Figura 4.6 (b) se muestra el espectro tras una transmisión óptica de $400 \mathrm{~m}$ MMF, siendo este el caso de mayor degradación por dispersión modal en el esquema propuesto. Se observa cómo se cumple el rechazo de $35 \mathrm{~dB}$ entre ambos sistemas UWB y WiMAX.

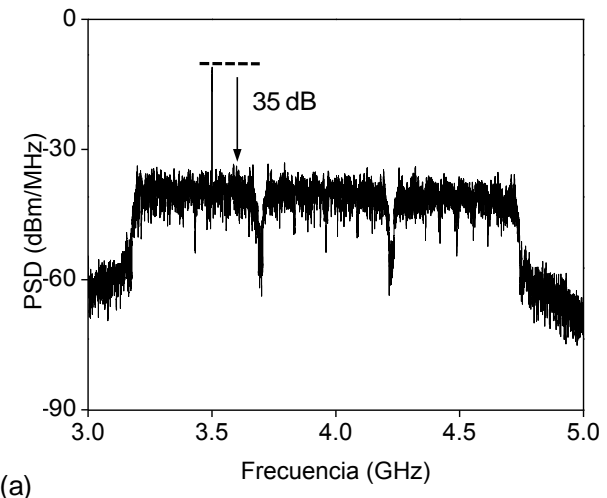

(a)

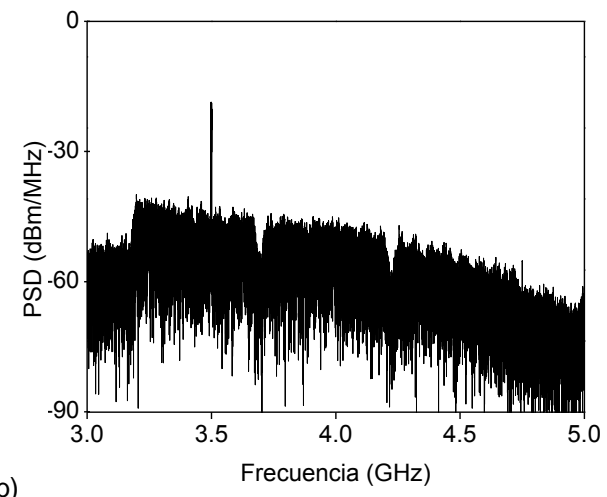

(b)

Figura 4.6. Densidad espectral de potencia de la señal conjunta UWB y WiMAX (a) antes del VCSEL y tras la transmisión óptica sobre (b) $400 \mathrm{~m}$ de MMF. RBW = 1 MHz.

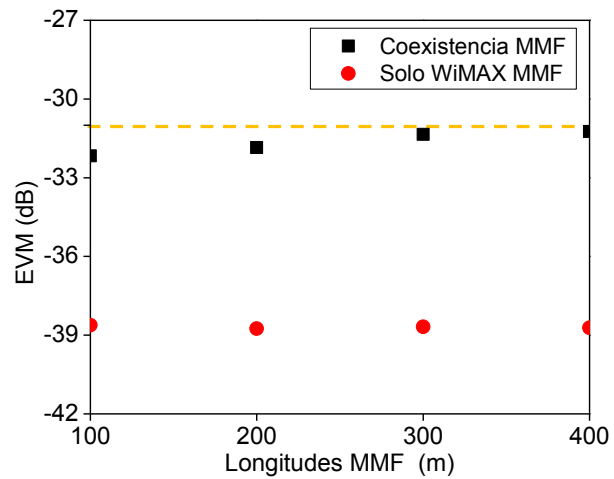

(a)

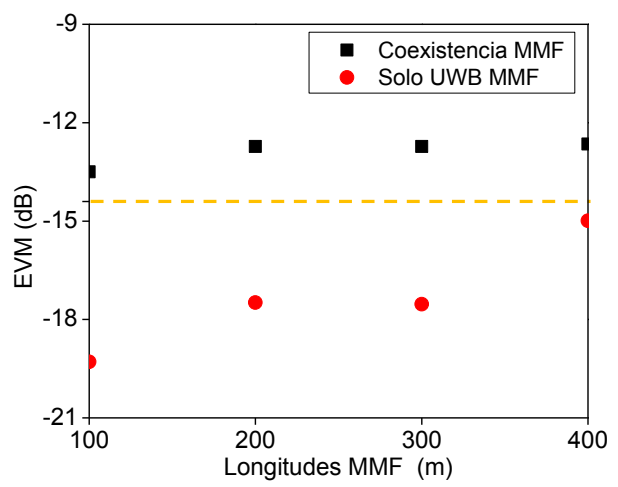

(b)

Figura 4.7. EVM medido para trasmisiones sobre diferentes longitudes MMF de (a) MB-OFDM UWB y (b) WiMAX 802.16d en el sistema propuesto.

El EVM mostrado en la Figura 4.7 se ha medido tras la conversión O/E de la señal óptica en el PIN/TIA, para la configuración RF adoptada para esta evaluación. En la Figura 4.7 (a), se muestra el EVM medido para una señal WiMAX 64 QAM en una transmisión RoMMF aislada y también para una transmisión RoMMF en presencia de MB-OFDM UWB TFC1 $200 \mathrm{Mbit} / \mathrm{s}$. Se observa cómo se produce una degradación en los valores de EVM por la presencia de UWB. Por ejemplo, para una longitud de $100 \mathrm{~m}$ 
MMF, los valores EVM en la situación WiMAX aislada son de $-38.6 \mathrm{~dB}$, mientras que aumentan hasta $-32.1 \mathrm{~dB}$ EVM WiMAX ante la presencia de UWB. A su vez, se observa cómo aumenta la distorsión lineal asociada a la dispersión modal por el aumento en la longitud del enlace MMF. Por ejemplo, para 400 m MMF de transmisión conjunta, se obtiene un valor de $-31.24 \mathrm{~dB}$ EVM WiMAX, mientras que para 100 m MMF, este valor es de -32.1 dB EVM WiMAX.

En la Figura 4.7 (b) el EVM UWB medido muestra cómo la presencia de WiMAX degrada de forma considerable la transmisión simultánea de MB-OFDM UWB en MMF. Para una longitud de $100 \mathrm{~m}$ MMF, el EVM medido en la situación UWB aislada es de $-19.1 \mathrm{~dB}$ EVM UWB, mientras que aumenta hasta $-13.5 \mathrm{~dB}$ EVM UWB para la situación de distribución conjunta. A su vez, el EVM UWB medido muestra la distorsión lineal asociada a la dispersión modal, la cual degrada el valor EVM para UWB conforme aumenta la longitud del enlace MMF.

Tras analizar la parte de transmisión óptica del sistema RoMMF para la transmisión simultánea de UWB y WiMAX, se ha evaluado el enlace radio. La evaluación del enlace radio UWB tras la transmisión óptica sobre $\mathrm{MMF}$, se ha realizado sobre distancias de $1 \mathrm{~m}, 2 \mathrm{~m}$ y $3 \mathrm{~m}$. El EVM medido para el enlace radio UWB obtenido se muestra en la Figura 4.8.

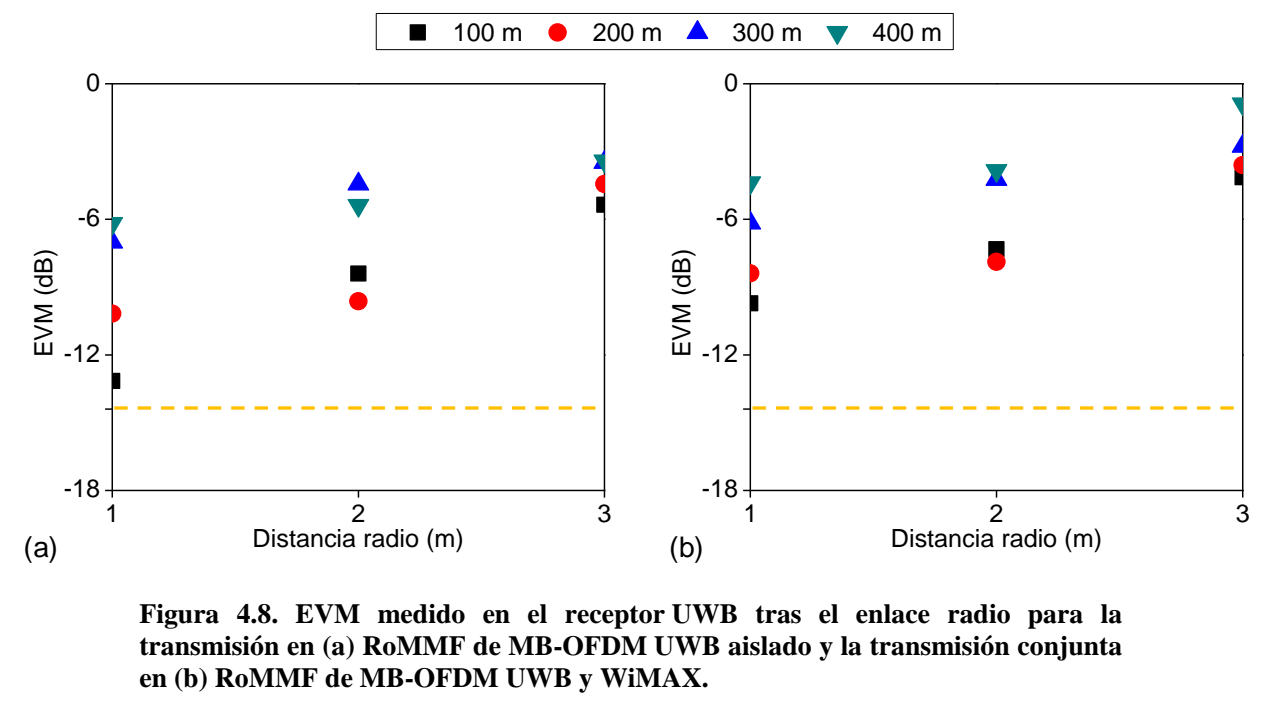

En la Figura 4.8 (a) se presenta el EVM medido cuando el sistema RoMMF implementa sólo la distribución de MB-OFDM UWB TFC1 200 Mbit/s. En la Figura 4.8 (b) se presenta el EVM medido al realizar una transmisión RoMMF conjunta con WiMAX 64 QAM. Comparando ambas figuras, se observa cómo el canal radio introduce 
distorsión en la comunicación UWB. Esta distorsión no es tan intensa como la debida a la transmisión óptica sobre MMF. Por ejemplo, una distribución de MB-OFDM UWB TFC1 $200 \mathrm{Mbit} / \mathrm{s}$ sobre un sistema RoMMF con $200 \mathrm{~m}$ MMF y un enlace radio de $2 \mathrm{~m}$, presenta un valor de EVM de $-9.62 \mathrm{~dB}$, mientras que si se transmite conjuntamente con WiMAX 64 QAM, se degrada hasta -7.88 dB EVM UWB.

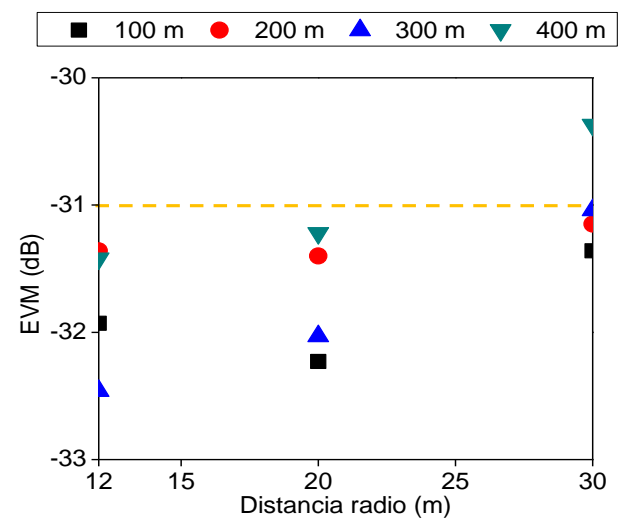

Figura 4.9. EVM medido en el receptor WiMAX tras el enlace radio para una transmisión conjunta de MB-OFDMUWB y WiMAX mediante un sistema RoMMF.

En la Figura 4.9 se muestra el EVM medido en el enlace radio WiMAX 802.16d. Estos valores indican la posibilidad de establecer un enlace radio WiMAX de hasta $30 \mathrm{~m}$ de alcance con una distribución óptica de 300 m MMF.

Los resultados experimentales obtenidos para la distribución simultánea de señales UWB y WiMAX mediante sistemas RoMMF, muestran la limitación en ancho de banda que supone la dispersión modal asociada a las de fibras MMF. A su vez, en el caso de una transmisión simultánea de WiMAX y UWB, el enlace UWB sufre una mayor degradación, como se observa en los valores de EVM UWB obtenidos. En cambio, el enlace WiMAX, presenta una degradación menor, permitiendo comunicaciones de hasta $300 \mathrm{~m}$ MMF con $30 \mathrm{~m}$ de enlace radio.

Por otra parte, en esta evaluación experimental se ha demostrado como las limitaciones de ancho de banda que presentan elementos como el VCSEL y el PIN/TIA han impuesto una distorsión muy notable que no permite la interoperabilidad efectiva entre UWB y WiMAX en RoMMF. Como solución se propone asegurar una mayor linealidad de los dispositivos E/O para rangos de frecuencias de trabajo altas y aumentar el ancho de banda de trabajo. 


\subsection{Análisis experimental de la coexistencia UWB y WiMAX en sistemas radio-sobre-fibra estándar monomodo}

La utilización de redes ópticas de acceso basadas en FTTx, permite la distribución simultánea de señales inalámbricas WiMAX y UWB sin necesidad de actualizar la parte de la transmisión óptica de las redes de acceso integradas. En concreto, las redes basadas en sistemas RoSSMF posibilitan la interconexión de múltiples escenarios inalámbricos.

La distribución de señales UWB mediante sistemas RoSSMF aparece reflejada en [Bel08] y [Llo08a]. En estas referencias, se expone el concepto de la utilización de sistemas RoF para el control de transceptores UWB. El objetivo es asegurar el acceso e interoperabilidad de los usuarios a contenidos de alto régimen binario como transmisiones de vídeo de alta definición mediante la conformación de una red UWB. A su vez, se define el concepto de pico-celda UWB como aquella donde un transceptor UWB conectado a una red basada en un sistema RoF, realiza las funciones de nodo de acceso UWB ante otros transceptores presentes en una WPAN de reducidas dimensiones. Este nodo de acceso UWB implementa operaciones de control de la interferencia tanto en la propia pico-celda como en pico-celdas vecinas. Esta red de nodos UWB posibilita la centralización de la gestión y la implementación de algoritmos de monitorización espectral en tiempo real en cada pico-celda. De esta forma, se asegura la interoperabilidad con otros sistemas inalámbricos presentes en la misma pico-celda.

Por otro lado, la utilización de sistemas RoSSMF para la distribución de WiMAX permite aplicaciones muy diversas que van desde la ampliación de la cobertura WiMAX hasta la centralización de la gestión de redes de sensores basadas en WiMAX. Por ejemplo, en [Mar07] se propone un sistema RoSSMF para la distribución de WiMAX IEEE 802.16d. En este sistema, se transmite la señal WiMAX mediante modulación directa de un láser DFB y se evalúan transmisiones WiMAX 64-QAM 3/4 con valores de -36.8 dB EVM para $5 \mathrm{~km}$ de fibra SSMF. La distribución de señales WiMAX en sistemas RoSSMF permite la reducción de equipamiento en la red desplegada, mejoras en la gestión de las comunicaciones y el acceso a servicios de elevado régimen binario a través de WiMAX en redes interiores. Estas ventajas se exponen en los trabajos recogidos en la publicación [Cor09] sobre las aplicaciones de la tecnología WiMAX en distribuciones sobre fibra.

\subsubsection{Distribución UWB mediante sistemas RoSSMF}

Las primeras aportaciones en el campo de la distribución de señales UWB en sistemas RoSSMF se realizaron en base a la tecnología IR-UWB, puesto que los 
primeros desarrollos civiles en tecnología radio UWB se basaron en técnicas de transmisión de pulsos ultra-cortos, como se comentó en el capítulo 2 de esta Tesis Doctoral.

En [Tan05] y [Ong06], se presentan resultados sobre la transmisión óptica de señales UWB en sistemas RoSSMF. En estos trabajos, se propone la transmisión de un sistema IR-UWB basado en un monociclo Gaussiano en la banda de $3 \mathrm{GHz}$ a $6 \mathrm{GHz}$ con longitudes de hasta $500 \mathrm{~m}$ de fibra SSMF. En [Kas08], se presenta un sistema IR-UWB a $1.25 \mathrm{Gbit} / \mathrm{s}$ distribuido sobre fibra SSMF que proporciona resultados de simulación con alcances de hasta $25 \mathrm{~km}$ SSMF con $1 \mathrm{~km}$ de fibra compensadora de la dispersión.

Sin embargo, se puede considerar [Llo08b] como la primera publicación que presenta resultados experimentales de una distribución de señales IR-UWB a $1.25 \mathrm{Gbit} / \mathrm{s}$ sobre una red FTTH, comparados con la implementación de un distribución de señales OFDM-UWB. Este trabajo, presenta la evaluación de un sistema de distribución de señales UWB a $1.25 \mathrm{Gbit} / \mathrm{s}$, tanto Multicarrier-OFDM UWB como IR-UWB, adecuadas para la transmisión de señales de audio y vídeo de alta definición sobre enlaces FTTH de fibra SSMF. Los resultados expuestos demuestran la posibilidad de realizar enlaces FTTH de hasta $50 \mathrm{~km}$ para transmitir señales UWB de $1.25 \mathrm{Gbit} / \mathrm{s}$.

Los resultados de las referencias [Tan05], [Ong06], [Kas08] y [Llo08b], muestran la viabilidad de la utilización de las redes basadas en sistemas RoSSMF para el transporte y la distribución de señales UWB, en este caso IR-UWB. Los dispositivos clave en los sistemas RoF ya han sido descritos con anterioridad, aunque cabe destacar la revisión de los mismos propuesta en [Jaz08]. En esta revisión, se ha mostrado el análisis de un sistema RoSSMF propuesto para distribuir IR-UWB y MB-OFDM UWB. A su vez, este análisis ha planteado que la modulación externa mediante MZM introduce mayor distorsión en las bandas de 3.1-5.1 GHz en comparación con láseres de modulación directa DFB. Al mismo tiempo, este trabajo ha mostrado cómo los sistemas MB-OFDM UWB son más adecuados para su transmisión óptica en SSMF que los IR-UWB, ya que estos últimos presentan mayor sensibilidad ante la distorsión no lineal. La distribución MB-OFDM UWB en sistemas RoSSMF también ha sido propuesta en [Llo08b] con señales OFDM-UWB no estándar de $1.25 \mathrm{Gbit} / \mathrm{s}$ para enlaces de hasta $50 \mathrm{~km}$ SSMF.

A continuación, y como paso previo a la evaluación de la coexistencia inalámbrica de MB-OFDM UWB y WiMAX, se muestra la evaluación experimental de un sistema de transmisión RoSSMF para la distribución de MB-OFDM UWB. 


\subsubsection{Prestaciones de la distribución MB-OFDM UWB en RoSSMF}

La evaluación de la distribución MB-OFDM UWB en sistemas RoSSMF se basa en el concepto descrito en la Figura 4.10. En esta figura se observa como la terminación óptica de línea (OLT, Optical Line Termination) se sitúa como parte final de la red óptica troncal y que además, suele asociarse a la central local que da servicio a un número dado de usuarios (CO, Central Office). Desde esta OLT, se distribuye la señal UWB a través de enlaces de fibra óptica de tipo SSMF, conformando una red FTTH, hasta llegar al equipamiento del cliente donde se encuentran las unidades ópticas de red (ONU, Optical Network Unit). Dependiendo de la distancia de transmisión en la red FTTH, se utilizan secciones de amplificación óptica o nodos remotos [Pra05b]. Cabe destacar que MB-OFDM UWB es una tecnología radio adecuada para la distribución en redes FTTH. Esto es debido a que las degradaciones que sufre en su transmisión óptica, tales como la dispersión cromática, la distorsión lineal intracanal o el ruido de fase no lineal, pueden ser compensadas mediante procesado electrónico en el receptor, como se propone en [Low07], [Jaz08].

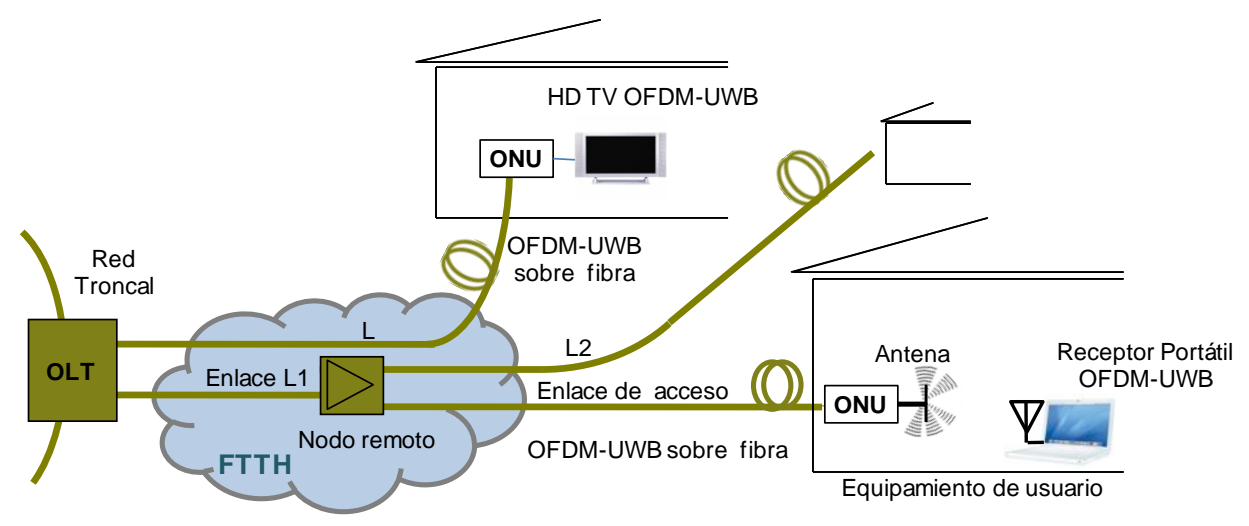

Figura 4.10. Esquema conceptual para la distribución de MB-OFDM UWB en redes FTTH mediante sistemas RoF.

En la evaluación experimental llevada a cabo en esta sección, se han propuesto dos arquitecturas FTTH claramente diferenciadas para la distribución óptica: (i) aquella que no incluye elementos ópticos activos en la transmisión, conocida como redes ópticas pasivas (PON, Passive Optical Network), y en la cual se han demostrado alcances de hasta $20 \mathrm{~km}$ SSMF [Jan07a], [Rer07], y (ii) aquella que incluye enlaces de fibra SSMF con amplificación óptica. En esta evaluación, se ha propuesto en primer lugar el estudio de la distribución MB-OFDM UWB tras su transmisión óptica mediante enlaces SSMF, y en segundo lugar, el estudio en recepción tras la foto-detección, filtrado, amplificación y posterior propagación radio hacia el usuario final. 


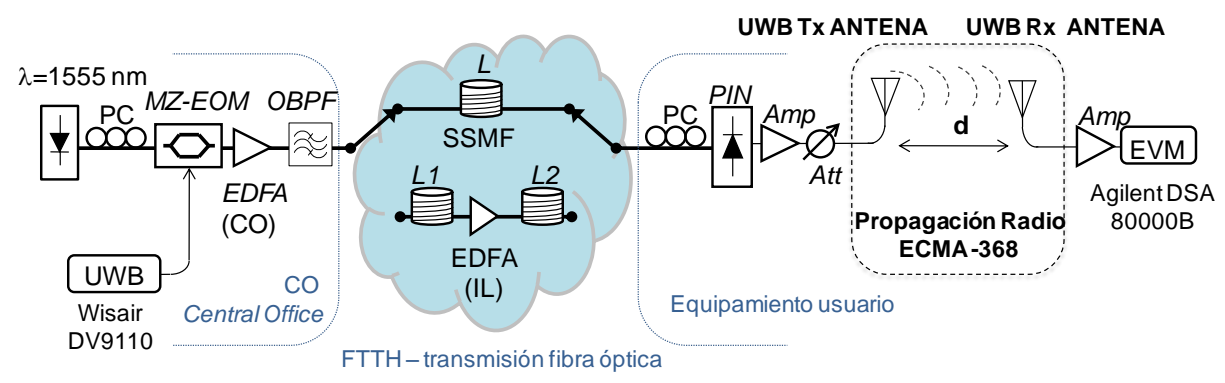

Figura 4.11. Esquema del montaje experimental para la evaluación de la distribución de MB-OFDM UWB en un sistema RoSSMF con enlaces FTTH.

En la Figura 4.11 se observa el montaje experimental propuesto en esta evaluación. En la sección CO, se ha implementado la generación de señales MB-OFDM UWB y posterior entrega a la red FTTH para su transporte. La señal MB-OFDM UWB ha sido generada mediante un módulo de evaluación UWB DV9110. Este módulo genera señales MB-OFDM UWB de acuerdo con el estándar ECMA-368 [ECMA368] para la banda de frecuencias BG1. En este montaje se han utilizado dos módulos DV9110, donde uno de ellos se asocia a un código TFC5 y el otro a un código TFC6 para BG1, denominados canal $\mathrm{Ch} 1$ y $\mathrm{Ch} 2$ respectivamente. El régimen binario implementado en cada canal es de $200 \mathrm{Mbit} / \mathrm{s}$, lo cual permite obtener un régimen binario agregado de $400 \mathrm{Mbit} / \mathrm{s}$ por usuario. El valor de $200 \mathrm{Mbit} / \mathrm{s}$ se elige por ser el régimen binario más elevado para la modulación QPSK en MB-OFDM UWB según [ECMA368]. Estos dos canales de señales UWB se han modulado ópticamente en un modulador Mach-Zehnder con tensión de media onda de $\mathrm{V}_{\pi}=4.5 \mathrm{~V}$ y con ancho de banda de trabajo analógico de $20 \mathrm{GHz}$. Para la modulación óptica de la señal UWB se ha utilizado un láser de cavidad externa (ECL, External Cavity Laser), centrado en una longitud de onda de $\lambda=1550 \mathrm{~nm}$ y que proporciona una potencia óptica de pico de unos $13 \mathrm{dBm}$. La señal modulada se ha inyectado a un amplificador óptico EDFA Amonics 30-B-FA, con una ganancia de $\mathrm{G}=23 \mathrm{~dB}$ para una potencia de saturación de $\mathrm{P}_{\mathrm{sat}}=13 \mathrm{dBm}$, que se denomina EDFA (CO) en la Figura 4.11. La señal óptica amplificada se ha filtrado mediante un filtro óptico paso banda (OBPF, Optical Band-Pass Filter) para reducir el ruido añadido por el EDFA fuera de la banda óptica de interés. El nivel de potencia óptica entregada a la fibra SSMF se ha controlado mediante el EDFA (CO). Este tipo de funcionalidad se incluye habitualmente en los OLT [Jan07b]. En la Tabla 4.3 se muestran los principales parámetros de la señal MB-OFDM UWB bajo estudio. 


\section{TABla 4.3. Parámetros de la SEÑal MB-OFDM UWB PARA EL SISTEMA RoSSMF BAJO ESTUDIO}

\begin{tabular}{c|c|c}
\hline \hline Canal & Ch1 & Ch2 \\
\hline TFC & TFC5 & TFC6 \\
\hline \multirow{2}{*}{ Banda de frecuencias } & Subbanda 1\# & Subbanda 2\# \\
\cline { 2 - 3 } & $\{3.168-3.696 \mathrm{GHz}\}$ & $\{3.696-4.224 \mathrm{GHz}\}$ \\
\hline Frecuencia central & $3.432 \mathrm{GHz}$ & $3.960 \mathrm{GHz}$ \\
\hline Régimen binario & \multicolumn{2}{|c}{$200 \mathrm{Mbit} / \mathrm{s}, \mathrm{QPSK}$} \\
\hline EIRP & \multicolumn{2}{|c}{$-41.3 \mathrm{dBm} / \mathrm{MHz}$} \\
\hline \hline
\end{tabular}

Posteriormente, la señal óptica generada se entrega a la sección de transmisión en fibra SSMF. Esta transmisión óptica se ha realizado sobre enlaces de $5 \mathrm{~km}, 10 \mathrm{~km}$ y $25 \mathrm{~km}$ de fibra SSMF. En un segundo caso, se han considerado dos enlaces de $25 \mathrm{~km}$ SSMF con amplificación óptica intermedia, mediante un EDFA intermedio (IL, Inline Amplification), que forman un enlace total de $50 \mathrm{~km}$ SSMF tal y como se observa en la Figura 4.11. Este EDFA intermedio tiene una ganancia de $\mathrm{G}=23 \mathrm{~dB}$ para una potencia de saturación de $\mathrm{P}_{\text {sat }}=13 \mathrm{dBm}$ y se denomina EDFA (IL) en la Figura 4.11. Cabe destacar que el efecto de la PMD en el caso de enlaces SSMF de hasta $25 \mathrm{~km}$ es muy residual. A partir de caracterizaciones realizadas experimentalmente en laboratorio mediante un analizador de redes ópticas Advantest Q7760, este valor de PMD es de tan solo $0.08 \mathrm{ps} / \mathrm{km}^{1 / 2}$ para la aproximación de primer orden. Otros factores como la GVD, que se presenta bajo el efecto de la supresión de portadora [Sch95], no son de importancia para la longitud de los enlaces SSMF evaluados.

Tras la transmisión óptica en los enlaces FTTH, la señal se ha entregado a la sección denominada equipamiento del cliente (CPE, Customer Premises Equipment). En esta sección, la señal se foto-detecta mediante un fotodiodo PIN con $0.7 \mathrm{~A} / \mathrm{W}$ de responsividad y ancho de banda de trabajo de hasta $45 \mathrm{GHz}$, U2T XPDV2040R. Posteriormente, la señal se ha amplificado para ajustar su EIRP en la antena UWB transmisora a $-41.3 \mathrm{dBm} / \mathrm{MHz}$, valor máximo de EIRP permitido según la regulación vigente [FCC02.48], [ETSI08]. El ajuste de la EIRP se ha realizado mediante un amplificador LNA con ganancia de $26 \mathrm{~dB}$ y un atenuador variable de $0 \mathrm{~dB}$ a $9 \mathrm{~dB}$, como se observa en la Figura 4.11. El controlador de polarización (PC, Polarization Controller) permite ajustar manualmente el estado de polarización de la señal óptica para maximizar la eficiencia del foto-detector. Estas funcionalidades se localizan habitualmente en la ONU en las redes FTTH [Jan07b]. El enlace radio se ha implementado mediante dos antenas omnidireccionales diseñadas para UWB de tipo patch In4Tel-omni con $0 \mathrm{dBi}$ de ganancia. Una vez transmitida vía radio, la señal MB-OFDM UWB es recibida y amplificada de nuevo para evaluar la calidad de la transmisión UWB mediante la medida del factor EVM en un analizador digital de 
señales DSA, Agilent DSA 80000B, con el software de procesado de señal VSA 89600. La reamplificación mediante un LNA con $\mathrm{G}=26 \mathrm{~dB}$ permite ajustar la señal MB-OFDM UWB recibida al margen dinámico del DSA.

En primer lugar, se han evaluado los resultados obtenidos para la distribución de la señal MB-OFDM UWB analizando únicamente las prestaciones de la transmisión óptica SSMF, sin considerar el enlace radio.

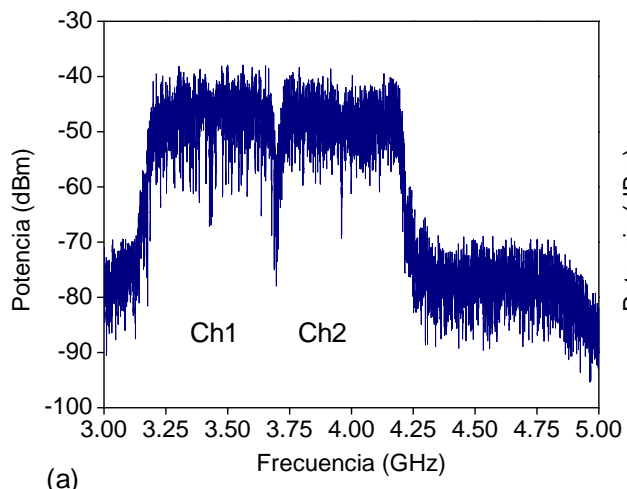

(a)

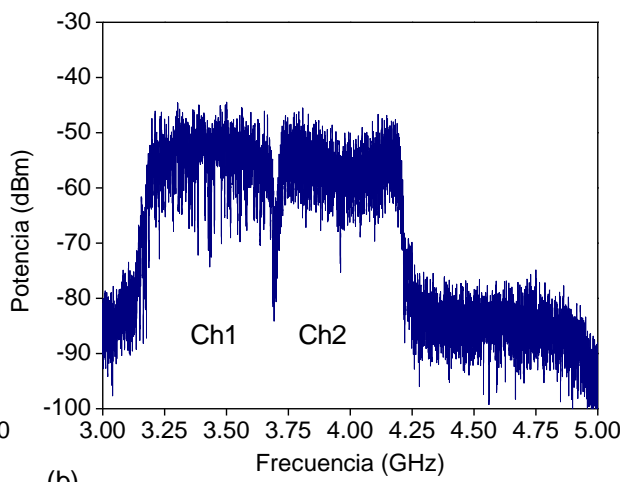

(b)

Figura 4.12. Densidad espectral de potencia de la señal MB-OFDM UWB (a) antes de su modulación óptica en el MZM y (b) tras su transmisión sobre un enlace de $25 \mathrm{~km}$ de fibra SSMF. RBW = 1 MHz.

En la Figura 4.12 se observa la densidad espectral de potencia de la señal MB-OFDM UWB generada. En la Figura 4.12 (a) se muestran los dos canales MB-OFDM UWB generados antes de su modulación óptica en el MZ de la Figura 4.11. En la Figura 4.12 (b) ya se observa la degradación introducida por la transmisión óptica de la señal MB-OFDM UWB sobre un enlace de $25 \mathrm{~km}$ SSMF.

Para evaluar la degradación introducida por la transmisión óptica y el impacto del ruido óptico introducido por el uso del EDFA (CO), se considera el factor EVM medido tras la foto-detección y ajuste de la señal antes de su radiación hacia el usuario final. El impacto del ruido óptico del EDFA (CO) se evalúa modificando el nivel de amplificación del mismo. A su vez, el EDFA (IL) considerado está activo para la configuración de $50 \mathrm{~km}$, pero con amplificación constante $\mathrm{G}=23 \mathrm{~dB}$. Las medidas de EVM se han realizado para los cuatro enlaces SSMF citados anteriormente de $5 \mathrm{~km}$, $10 \mathrm{~km}, 25 \mathrm{~km}$ y $50 \mathrm{~km}$, y también para una configuración sin tramos de fibra SSMF, es decir, para una configuración B2B. 


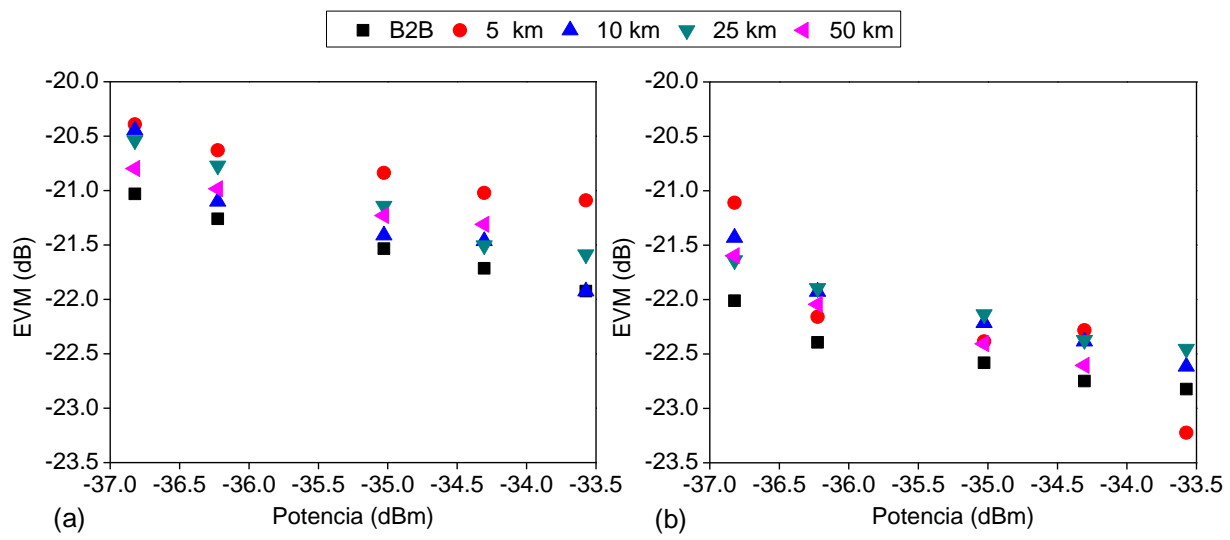

Figura 4.13. EVM UWB medido para diferentes distancias SSMF y potencias ópticas del EDFA (CO) para (a) Ch1 y (b) Ch2.

En la Figura 4.13 se presentan las medidas del factor EVM obtenidas para la señal MB-OFDM UWB en las diferentes distancias SSMF. Se observa que el valor de EVM UWB en el Ch1 UWB varía entre $-20.75 \mathrm{~dB}$ y $-22 \mathrm{~dB}$, y en el caso del Ch2, varía entre $-21 \mathrm{~dB}$ y $-23 \mathrm{~dB}$. El aumento de la potencia del EDFA (CO), que se presenta como potencia foto-detectada en el eje de abscisas, no es un factor limitante del comportamiento de la transmisión, puesto que el EVM UWB medido siempre se sitúa por debajo del umbral recomendado en [ECMA368] para garantizar una comunicación UWB, es decir, -14.4 dB EVM.

A continuación, se presentan el EVM UWB medido en la evaluación de la propagación radio de la señal MB-OFDM UWB, pero sin ningún tipo de modulación óptica ni de transmisión en fibra, únicamente conectando los módulos a las antenas para evaluar la propagación en el canal radio. En este caso, el EVM medido se muestra en la Tabla 4.4. Se observa cómo los valores EVM para el Ch2 UWB son peores que para el Ch1 UWB debido a que la respuesta en frecuencia que presentan los amplificadores usados para ajustar la potencia transmitida, así como la respuesta de las antenas, no es constante para toda la banda de frecuencias UWB.

\section{TABLA 4.4. EVM MEDIDO EN EL SISTEMA MB-OFDM UWB PROPUESTO SIN TRANSMISIÓN ÓPTICA}

\begin{tabular}{l|c|c|c|c}
\hline \hline & $\mathbf{d = 0 . 5} \mathbf{~ m}$ & $\mathbf{d = 1} \mathbf{~ m}$ & $\mathbf{d = 1 . 5} \mathbf{~ m}$ & $\mathbf{d = 2} \mathbf{~ m}$ \\
\hline EVM Ch1 & $-20.39 \mathrm{~dB}$ & $-17.81 \mathrm{~dB}$ & $-14.6 \mathrm{~dB}$ & $-13.18 \mathrm{~dB}$ \\
\hline EVM Ch2 & $-18.63 \mathrm{~dB}$ & $-15.79 \mathrm{~dB}$ & $-12.72 \mathrm{~dB}$ & $-10.37 \mathrm{~dB}$ \\
\hline \hline
\end{tabular}

En la Figura 4.14 (a), se observa la densidad espectral de potencia asociada al espectro de MB-OFDM UWB recibido tras $25 \mathrm{~km}$ de transmisión sobre SSMF y una 
propagación radio sobre una distancia de $1.5 \mathrm{~m}$. Al comparar este espectro con el de la Figura 4.12 (b), se observa la distorsión introducida por el canal de propagación radio. Las constelaciones asociadas a cada canal MB-OFDM UWB se muestran en la Figura 4.14 (b) y (c) tras $25 \mathrm{~km}$ de fibra SSMF, y en la Figura 4.14 (d) y (e) para $25 \mathrm{~km}$ SSMF con un enlace radio de $1.5 \mathrm{~m}$. En estas figuras se observa como el $\mathrm{Ch} 2$ sufre una distorsión superior a la mostrada por el Ch1 tras su propagación radio, como consecuencia de la respuesta en frecuencia de los amplificadores y antenas usados en esta evaluación.

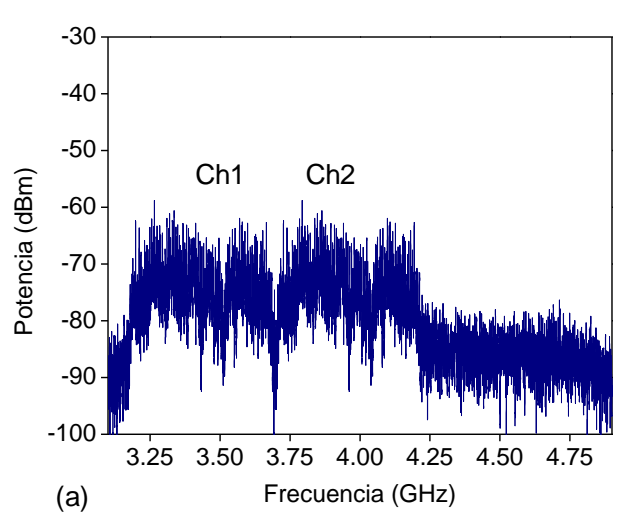

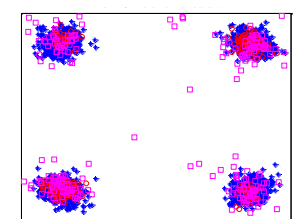

(b)

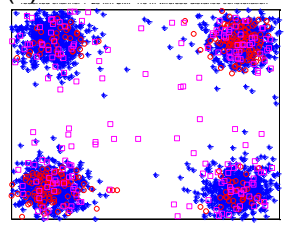

(d)

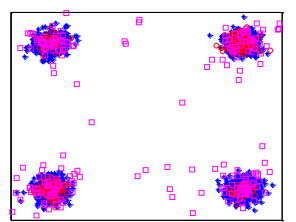

(c)

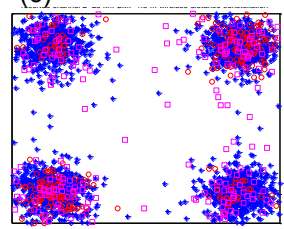

(e)

Figura 4.14. (a) Espectro de la señal MB-OFDM UWB recibida tras un enlace de $25 \mathrm{~km}$ SSMF con $1.5 \mathrm{~m}$ de propagación radio. $\mathrm{RBW}=1 \mathrm{MHz}$. Constelaciones asociadas a MB-OFDM UWB (b) Ch1 y (c) Ch2 para una transmisión de $25 \mathrm{~km}$ SSMF sin enlace radio. Constelaciones asociadas a MB-OFDM UWB (d) Ch1 y (e) Ch2 tras un enlace $25 \mathrm{~km}$ SSMF con $1.5 \mathrm{~m}$ de propagación radio.

Los valores EVM UWB mostrados en la Figura 4.15 se han medido para la señal MB-OFDM UWB recibida tras un enlace radio para distancias de $0 \mathrm{~m}, 0.5 \mathrm{~m}, 1 \mathrm{~m}$, $1.5 \mathrm{~m}, 2 \mathrm{~m}, 2.5 \mathrm{~m}$ y $3 \mathrm{~m}$. El EDFA (CO) se ha configurado con una potencia óptica fija cuyo valor en foto-detección es $-34.3 \mathrm{dBm}$. Es posible extender las conclusiones aquí mostradas a otras potencias ópticas siguiendo el comportamiento expresado por los resultados de la Figura 4.13. En el EVM UWB representado en la Figura 4.15 se muestra el umbral EVM UWB de $-14.4 \mathrm{~dB}$ como una línea discontinua. Como se observa en la figura, el EVM UWB de Ch1 y Ch2, teniendo en cuenta una misma distancia radio, se mantiene en unos valores muy constantes para los diferentes enlaces SSMF. Esto indica que en la distribución RoF evaluada, la transmisión en SSMF no es un factor limitante comparada con la limitación impuesta por la propagación radio. 


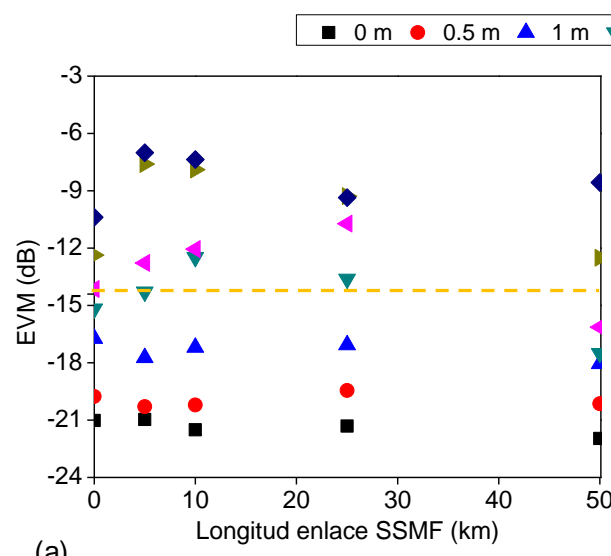

(a)

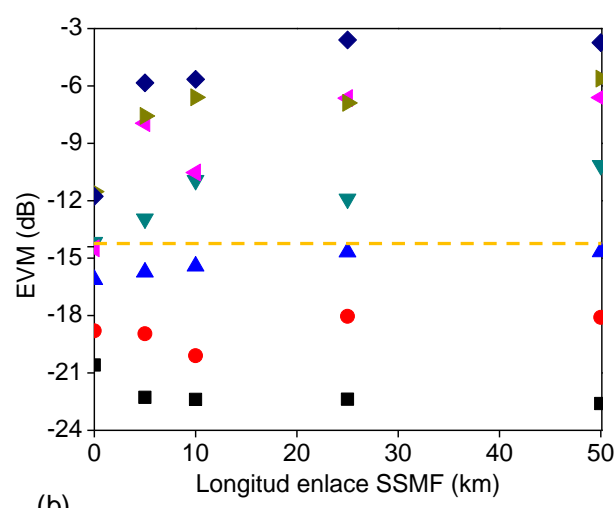

(b)

Figura 4.15. EVM UWB medido según diferentes distancias de propagación radio para cada enlace SSMF para una potencia óptica entregada de $\mathbf{- 3 4 . 3 1} \mathbf{d B m}$ en foto-detección para MB-OFDM UWB (a) Ch1 y (b) Ch2.
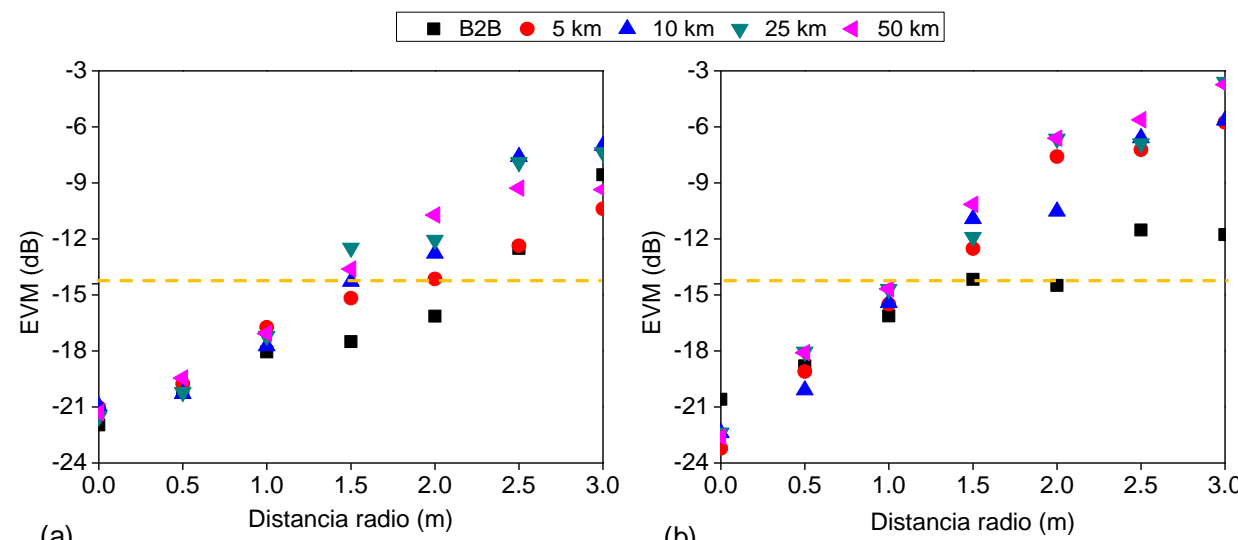

(b)

Figura 4.16. EVM UWB medido según diferentes enlaces SSMF para diferentes distancias de propagación radio con una potencia óptica entregada de $\mathbf{- 3 4 . 3 1} \mathrm{dBm}$ en foto-detección para MB-OFDM UWB (a) Ch1 y (b) Ch2.

En la Figura 4.16, se ha representado el EVM UWB medido en recepción para cada enlace SSMF y B2B en función de la distancia de propagación radio. En el caso del Ch1, los valores EVM UWB están por debajo del umbral EVM UWB para una distancia radio de $1 \mathrm{~m}$ para todas las configuraciones de SSMF, mientras que esta distancia aumenta hasta los $2 \mathrm{~m}$ sólo para la configuración de $5 \mathrm{~km}$ de enlace SSMF. Por otra parte, en el caso de $\mathrm{Ch} 2$, se limita el alcance radio hasta $1 \mathrm{~m}$ para cualquier distancia SSMF. Si se comparan los resultados B2B con los obtenidos con transmisión en fibra, se observa como en Ch1 y Ch2 existe una reducción del alcance radio máximo de $1 \mathrm{~m}$, 
puesto que para la configuración B2B se obtiene un alcance radio máximo de $2 \mathrm{~m}$ y para la configuración de $50 \mathrm{~km}$ SSMF, tan sólo se obtiene $1 \mathrm{~m}$ de alcance radio.

El EVM medido en esta evaluación de la distribución de señales MB-OFDM UWB en un sistema RoSSMF con posterior propagación radio, indican que la comunicación UWB es factible para un alcance radio de $1 \mathrm{~m}$ para una distribución en fibra de $50 \mathrm{~km}$, y para un alcance radio de hasta $1.5 \mathrm{~m}$ para $10 \mathrm{~km}$ de fibra SSMF. A su vez, se observa que la transmisión sobre SSMF degrada la relación señal a ruido (SNR, Signal-to-Noise Ratio) y reduce el alcance radio máximo del sistema, $2 \mathrm{~m}$ para la configuración B2B y tan sólo $1 \mathrm{~m}$ para la configuración de 50 km SSMF. Comparando el EVM medido tras una propagación radio de $1.5 \mathrm{~m}$, se observa una degradación de $2.13 \mathrm{~dB}$ para el Ch1 y de $2.5 \mathrm{~dB}$ EVM para el Ch2 entre el sistema RoSSMF con configuración B2B y la configuración de $50 \mathrm{~km}$ SSMF de enlace óptico.

\subsubsection{Prestaciones de la distribución en coexistencia RoSSMF sobre PON}

En esta sección, se evalúa la distribución conjunta de MB-OFDM UWB y WiMAX IEEE 802.16e para un sistema RoSSMF de tipo PON, es decir, que no incluye secciones ópticas activas, en este caso amplificadores ópticos intermedios.

Como ya se ha expuesto en el capítulo 2 de esta Tesis Doctoral, las redes PON son parte de la tecnología de redes ópticas de acceso FTTH. A su vez, las redes PON protagonizan un papel muy importante en el actual despliegue de las redes ópticas de acceso [Far08]. El uso de la tecnología PON es predominante en áreas con un gran número de usuarios [Koo07]. El despliegue de redes PON tiene un coste económico menor que otras tecnologías ópticas de acceso punto-a-punto debido a diferentes factores: (i) en las redes PON no se requiere compensar ciertos factores de degradación en la transmisión óptica como PMD o GVD [Shu07]; (ii) el aumento e inclusión de nuevos usuarios a la red PON se realiza mediante el conexionado pasivo de nuevas fibras, aportando una mayor flexibilidad y escalabilidad que otras redes ópticas de acceso, que al requerir amplificación óptica intermedia necesitan una planificación de la red mucho más compleja; (iii) el mantenimiento de las redes PON tiene un coste más bajo que otras redes de acceso debido a su facilidad de mantenimiento al no tener elementos activos en la red. Actualmente, las redes PON estudiadas presentan alcances de hasta $20 \mathrm{~km}$ de fibra SSMF [Rer07]. El uso de redes tipo PON para la distribución simultánea de UWB y WiMAX, permite una reducción de los dispositivos activos de toda la red, y por lo tanto una disminución de los costes del despliegue de las mismas. 


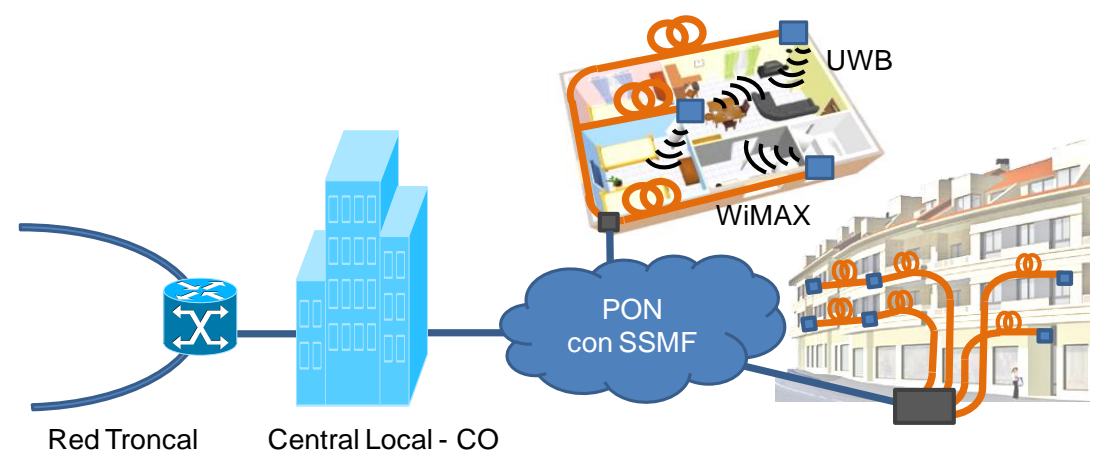

Figura 4.17. Concepto de la distribución simultánea de servicios inalámbricos basados en WiMAX y UWB mediante el uso de sistemas RoSSMF en redes PON.

El concepto de distribución simultánea MB-OFDM UWB y WiMAX IEEE 802.16e en un sistema RoF de tipo PON se presenta en la Figura 4.17. En esta figura, se observa una central local (CO) que genera señales UWB y WiMAX acordes con los servicios que el operador va a desplegar para los usuarios finales. La transmisión simultánea de ambas señales se realiza sobre una red óptica de acceso basada en SSMF, una red PON en este caso. En el equipamiento de usuario se foto-detecta, amplifica y radia cada servicio inalámbrico hacia el dispositivo adecuado en el usuario final.

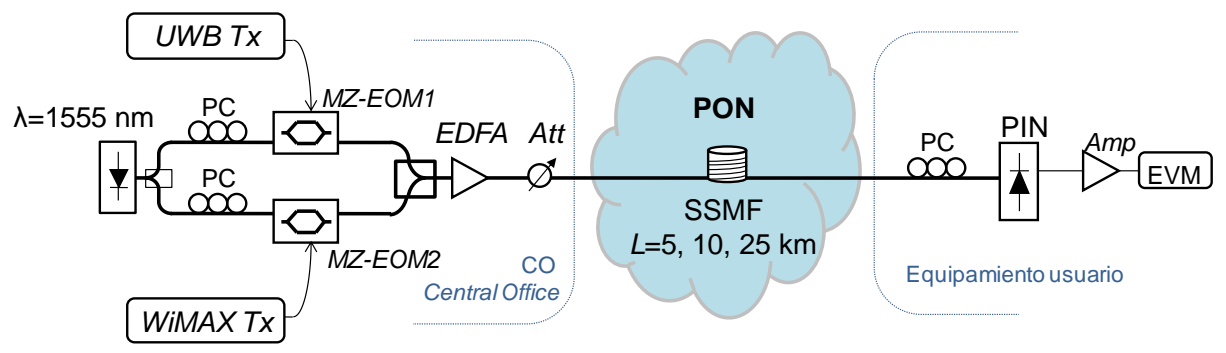

Figura 4.18. Esquema del montaje experimental realizado para la evaluación de la distribución simultánea de WiMAX y UWB en una red PON mediante un sistema RoSSMF.

Para evaluar el concepto de distribución simultánea de MB-OFDM UWB y WiMAX 802.16e en sistemas RoSSMF PON, se ha realizado el montaje experimental mostrado en la Figura 4.18. Este montaje se ha divido en tres secciones. La primera sección o CO, contempla la generación de los servicios inalámbricos MB-OFDM UWB y WiMAX 802.16e para su conversión E/O y su posterior distribución en una red PON. La señal MB-OFDM UWB se ha implementado mediante el módulo UWB DV9110 de acuerdo con el estándar [ECMA368]. En esta evaluación, se han definido dos configuraciones UWB. En la primera configuración, se han utilizado dos módulos DV9110, donde uno de ellos se asocia a un código TFC5 y el otro a un código TFC6 
para BG1, denominados canal $\mathrm{Ch} 1$ y $\mathrm{Ch} 2$ respectivamente. Estos canales proporcionan un régimen binario de $200 \mathrm{Mbit} / \mathrm{s}$ y un régimen agregado de $400 \mathrm{Mbit} / \mathrm{s}$. La segunda configuración MB-OFDM UWB ha implementado una señal UWB asociada a un código de salto en frecuencia TFC1 para BG1. Esta configuración proporciona un régimen binario de $200 \mathrm{Mbit} / \mathrm{s}$. Los principales parámetros de las dos configuraciones MB-OFDM UWB implementadas se muestran en la Tabla 4.5.

TABLA 4.5. PARÁMETROS MB-OFDM UWB PARA LA DISTRIBUCIÓN CONJUNTA EN UNA RED PON

\begin{tabular}{c|c|c}
\hline \hline TFC & TFC1 & TFC5 -TFC6 \\
\hline \multirow{3}{*}{ Banda de frecuencias } & BG1 & Subbanda 1\# y 2\# en BG1 \\
\cline { 2 - 3 } & $\{3.168-4.752 \mathrm{GHz}\}$ & $\begin{array}{c}\{3.168-3.696 \mathrm{GHz}\} \\
\{3.696-4.224 \mathrm{GHz}\}\end{array}$ \\
\hline \multirow{2}{*}{ Frecuencia central } & $3.960 \mathrm{GHz}$ & $\begin{array}{l}3.432 \mathrm{GHz} \\
\end{array}$ \\
\hline Régimen binario & \multicolumn{2}{|c}{$200 \mathrm{Mbit} / \mathrm{s}, \mathrm{QPSK}$} \\
\hline EIRP & \multicolumn{2}{|c}{$-41.3 \mathrm{dBm} / \mathrm{MHz}$} \\
\hline \hline
\end{tabular}

La señal WiMAX se ha implementado de acuerdo al estándar IEEE 802.16e [IEEE802.16e] mediante un generador de señales vectoriales, Agilent ESG E4438C y un software de procesado de señales. La frecuencia central de la señal WiMAX es $3.5 \mathrm{GHz}$, y se han considerado anchos de banda nominales de $5 \mathrm{MHz}, 10 \mathrm{MHz}$ y $20 \mathrm{MHz}$. En la Tabla 4.6 se muestran los principales parámetros de la señal WiMAX 802.16e.

TABla 4.6. Parámetros WiMAX IEEE 802. 16E PARA La DISTRIBUCIÓN CONJUNTA EN UNA RED PON

\begin{tabular}{l|c|c|c}
\hline \hline Frecuencia central & \multicolumn{3}{|c}{$3.5 \mathrm{GHz}$} \\
\hline Ancho de banda nominal & $5 \mathrm{MHz}$ & $10 \mathrm{MHz}$ & $20 \mathrm{MHz}$ \\
\hline Espaciado subportadoras & $5.46875 \mathrm{kHz}$ & $10.9375 \mathrm{kHz}$ & $21.875 \mathrm{kHz}$ \\
\hline $\begin{array}{l}\text { Duración de símbolo } \\
\text { (Ts=1/Espaciado subportadora) }\end{array}$ & $182.86 \mu \mathrm{s}$ & $102.85 \mu \mathrm{s}$ & $57.14 \mu \mathrm{s}$ \\
\hline Sobremuestreo & \multicolumn{3}{|c}{$24 / 28$} \\
\hline Periodo de guarda (G) & \multicolumn{3}{|c}{ QPSK (1/2 CTC) } \\
\hline Modulación & \multicolumn{3}{|c}{} \\
\hline Régimen binario DL & $3.17 \mathrm{Mbit} / \mathrm{s}$ & $6.34 \mathrm{Mbit} / \mathrm{s}$ & $12.68 \mathrm{Mbit} / \mathrm{s}$ \\
\hline \hline
\end{tabular}

La CO implementa la modulación de las señales RF en una sola longitud de onda óptica mediante un láser ECL centrado en $\lambda=1550 \mathrm{~nm}$, y el uso de dos moduladores 
externos Mach-Zehnder funcionando en la región lineal de trabajo o QB. La señal modulada ópticamente se amplifica mediante un EDFA Amonics 30-B-FA, con una ganancia de $G=23 \mathrm{~dB}$ para una potencia de saturación de $\mathrm{P}_{\text {sat }}=13 \mathrm{dBm}$. La potencia óptica entregada a la entrada de la red PON se controla mediante un atenuador óptico, que permite evaluar la degradación introducida por el aumento de potencia óptica en el sistema. Para ello, se mantiene constante la potencia entregada por el EDFA y se varía el atenuador óptico para obtener valores de potencia entregada a la red PON de $\{-3,-1,1,3,5,7\} \mathrm{dBm}$, lo cual permite a su vez, mantener constante el ruido introducido por el EDFA.

La sección de transmisión óptica PON está compuesta por enlaces de fibra SSMF de $5 \mathrm{~km}, 10 \mathrm{~km}$ y $25 \mathrm{~km}$.

Finalmente, la señal óptica se transmite hasta el equipamiento de usuario. En esta sección, la señal óptica se foto-detecta mediante un PD con una responsividad de $0.7 \mathrm{~A} / \mathrm{W}$ y ancho de banda de trabajo de $45 \mathrm{GHz}$ que permite recuperar la señal de banda ultra-ancha. Una vez foto-detectada, la señal se amplifica de nuevo para evaluar la calidad de la transmisión UWB y WiMAX mediante la medida del factor EVM en un analizador digital de señales DSA, Agilent DSA 80000B, con el software de procesado de señal VSA 89600.
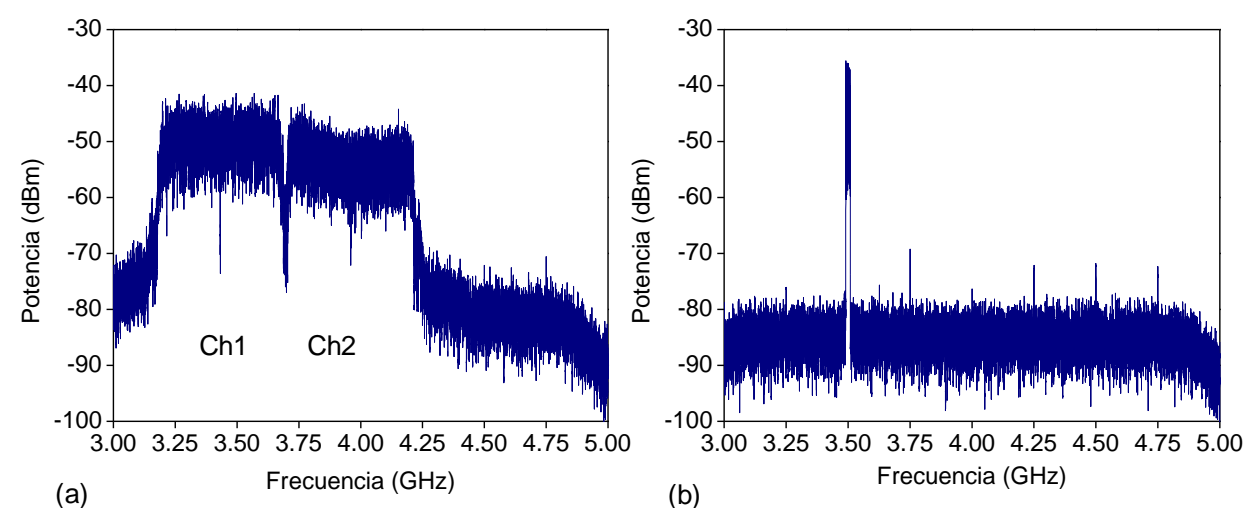

Figura 4.19. Densidad espectral de potencia de la transmisión aislada (a) de la señal MB-OFDM UWB Ch1 y Ch2, y (b) de la señal WiMAX 20 MHz BW en una red PON bajo configuración B2B. RBW $=1 \mathrm{MHz}$.

En la Figura 4.19 (a) se muestra el espectro de la señal MB-OFDM UWB generada con Ch1 y Ch2. La Figura 4.19 (b) muestra el espectro de la señal WiMAX para un ancho de banda nominal de $20 \mathrm{MHz}$. En este caso, se observa cómo coincide espectralmente la subbanda 1\#, donde se ubica el Ch1 de la canalización MB-OFDM UWB, con la señal WiMAX. Ambos espectros han sido detectados tras su 
modulación E/O individual en MZ-EOM1 y MZ-EOM2 de la Figura 4.18, sin realizar ninguna transmisión en fibra.

En primer lugar se ha evaluado el comportamiento de los enlaces SSMF PON para una distribución aislada de MB-OFDM UWB. Para ello, se han evaluado las dos configuraciones UWB propuestas en el esquema de la Figura 4.18, donde únicamente la parte del transmisor UWB, UWB Tx, se encuentra activa. La transmisión óptica ha sido evaluada para un rango de potencias ópticas entregadas a la fibra de $\{0,2,4,6\} \mathrm{dBm}$.

En la Figura 4.20, se representan el EVM medido para una transmisión MB-OFDM UWB aislada. En la Figura 4.20 (a) y en la Figura 4.20 (b), se observa cómo la degradación introducida por las diferentes potencias ópticas para cada distancia SSMF es menor de $2 \mathrm{~dB}$ EVM UWB. Los EVM medidos para ambas configuraciones UWB, siempre se sitúan por debajo del umbral de -14.4 dB EVM que asegura la comunicación MB-OFDM UWB, según [ECMA368].

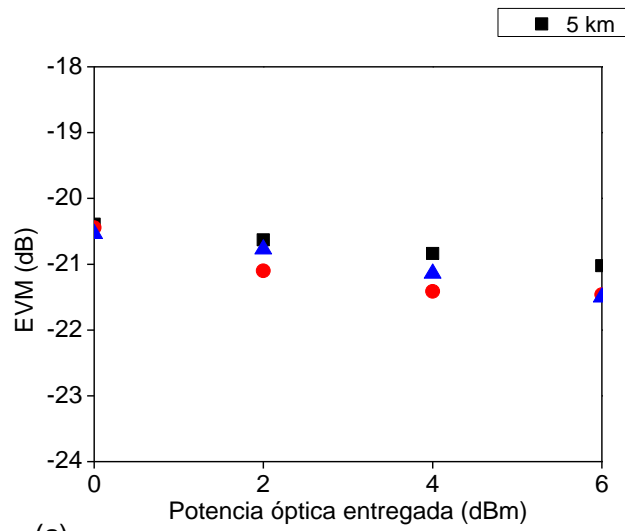

(a)

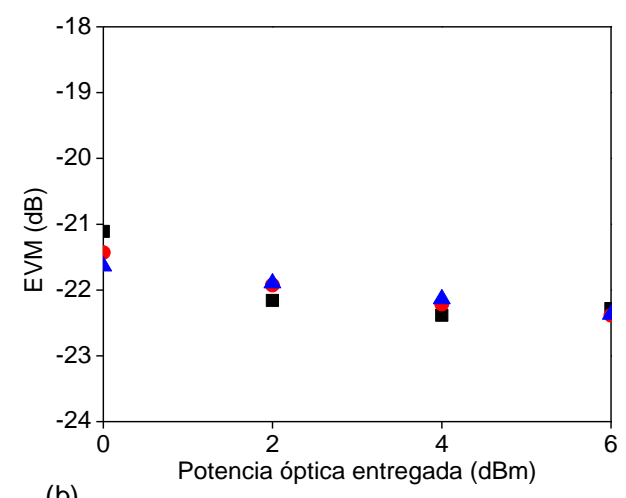

(b)

Figura 4.20. EVM medido para la distribución aislada de MB-OFDM UWB (a) Ch1 y (b) Ch2 para diferentes potencias ópticas entregadas a la red PON propuesta sobre cada enlace SSMF bajo estudio.

En la Figura 4.21, se representa el EVM medido para la distribución aislada de las diferentes configuraciones WiMAX 802.16e. Se observa que la degradación introducida por el aumento de la longitud del enlace SSMF de $10 \mathrm{~km}$ a $25 \mathrm{~km}$ es menor de 2 dB EVM WiMAX. 

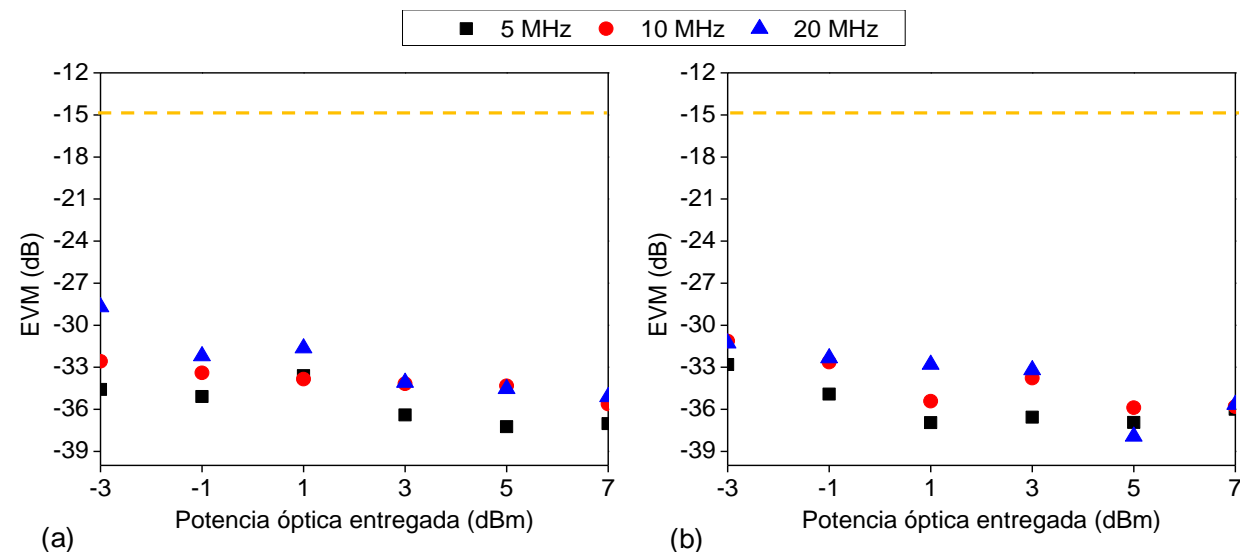

Figura 4.21. EVM medido para la distribución aislada de las diferentes configuraciones WiMAX 802.16e para enlaces SSMF de (a) $10 \mathrm{~km}$ y (b) $25 \mathrm{~km}$ para diferentes potencias ópticas entregadas a la red PON propuesta.

La evaluación del impacto de WiMAX 802.16e sobre MB-OFDM UWB en términos de EVM UWB se presenta en la Figura 4.22. Se observa cómo ante la presencia de WiMAX, la configuración MB-OFDM UWB TFC1 sufre una degradación menor que la configuración UWB Ch1 y Ch2, para cualquier configuración WiMAX 802.16e. A su vez, se observa cómo conforme aumenta el ancho de banda nominal de WiMAX 802.16e, los valores EVM UWB para cada configuración empeoran levemente pero no de forma significativa. La presencia de WiMAX se traduce en una degradación más intensa para UWB Ch1 que para UWB Ch2, debido a que $\mathrm{Ch} 2$ sufre la coexistencia espectral de forma adyacente y no de forma permanente como Ch1.

La distribución simultánea de WiMAX 10 MHz BW con MB-OFDM UWB Ch1 y Ch2, introduce una degradación de $3.2 \mathrm{~dB}$ EVM UWB para $1 \mathrm{dBm}$ de potencia óptica entregada a la entrada del enlace de $25 \mathrm{~km} \mathrm{SSMF}$, como se observa comparando los valores EVM UWB mostrados en la Figura 4.22 (b), (e) y Figura 4.20 (a), (b). 

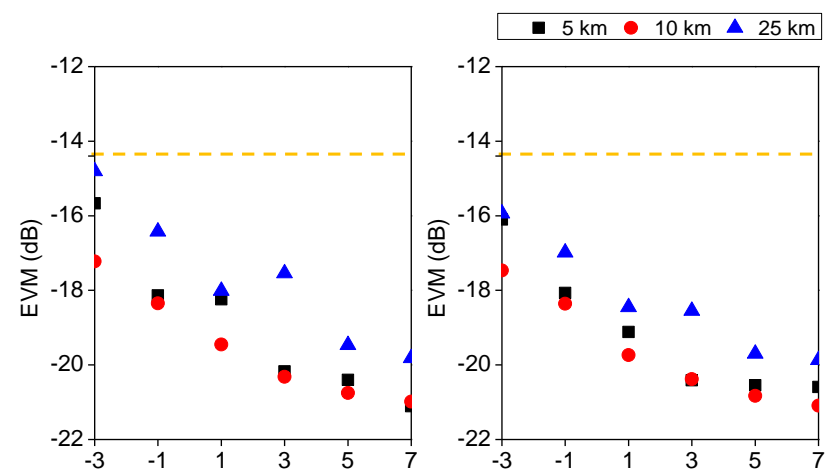

(a) Potencia óptica entregada (dBm)

(b) Potencia óptica entregada $(\mathrm{dBm})$
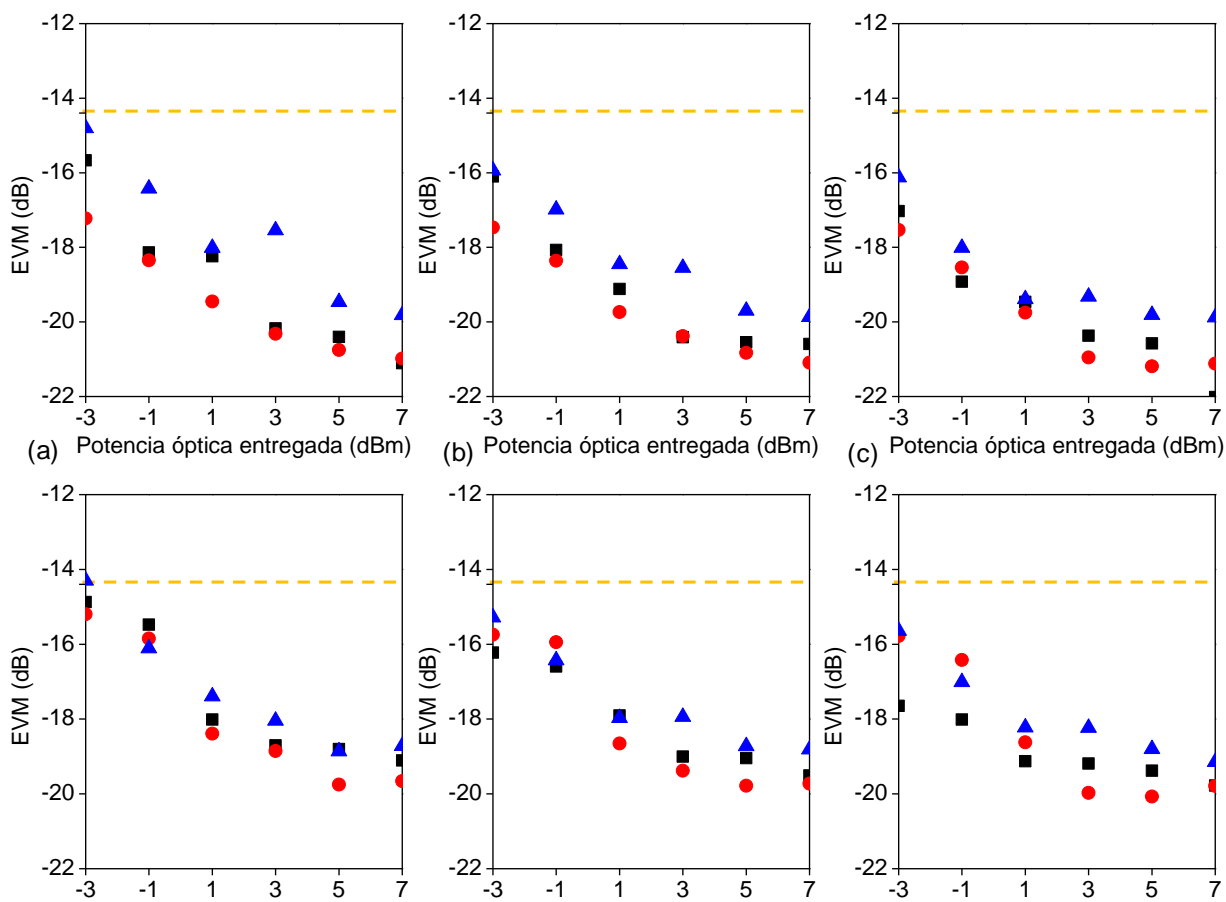

(c) Potencia óptica entregada $(\mathrm{dBm})$

(d) Potencia óptica entregada $(\mathrm{dBm})$

(e) Potencia óptica entregada $(\mathrm{dBm})$
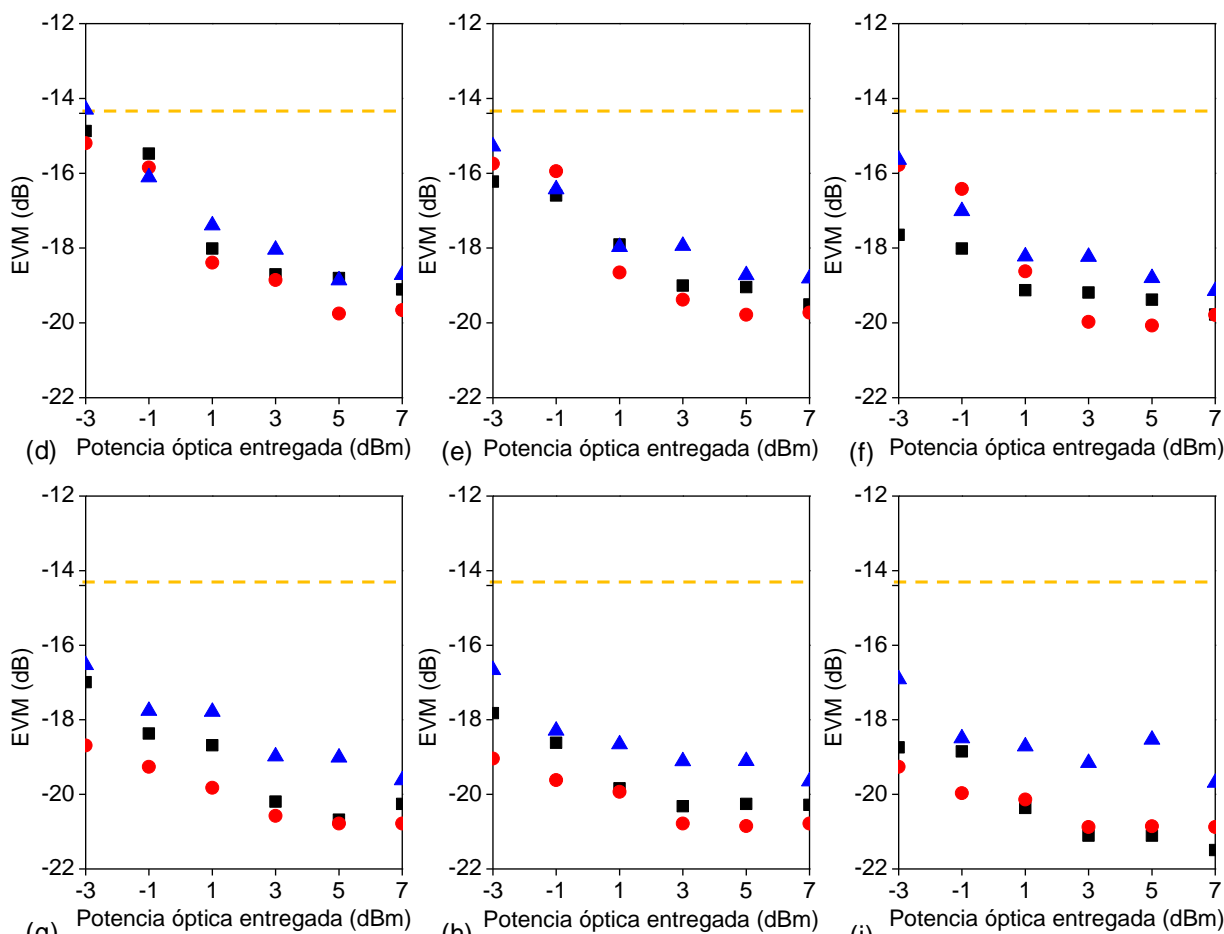

(f) Potencia óptica entregada $(\mathrm{dBm})$

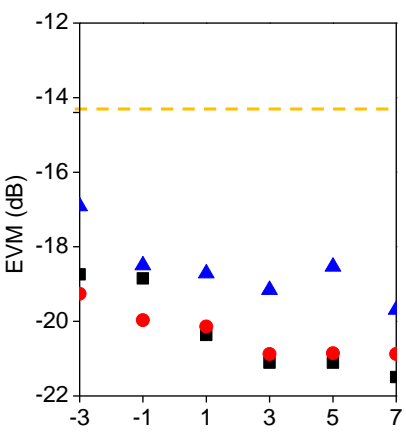

(h)

(i) Potencia óptica entregada $(\mathrm{dBm})$

Figura 4.22. EVM UWB medido para una distribución conjunta de MB-OFDM UWB y WiMAX 802.16e en la red PON propuesta para las configuraciones: UWB Ch1 con WiMAX (a) $5 \mathrm{MHz}$ (b) $10 \mathrm{MHz}$ y (c) $20 \mathrm{MHz}$ BW, UWB Ch2 con WiMAX (d) $5 \mathrm{MHz}$, (e) $10 \mathrm{MHz}$ y (f) $20 \mathrm{MHz}$ BW, y por último UWB TFC1 con WiMAX (g) 5 MHz (h) 10 MHz y (i) 20 MHz BW. 


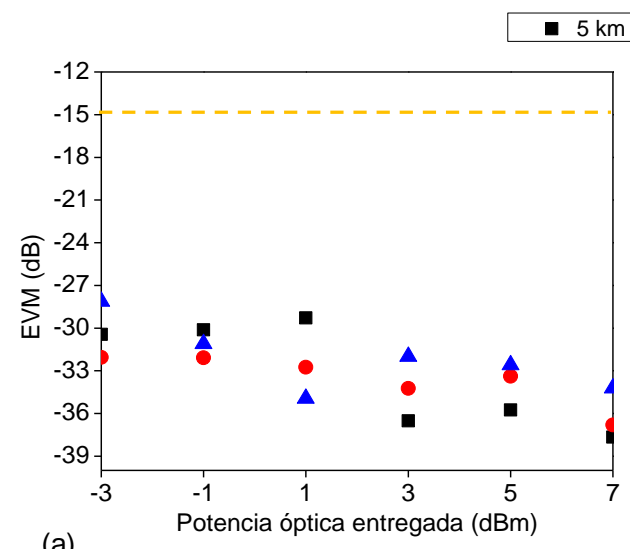

(a)

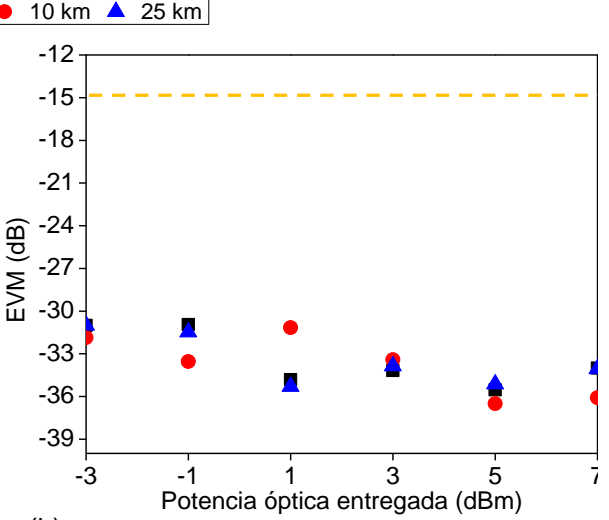

(b)

Figura 4.23. EVM WiMAX medido para una distribución conjunta MB-OFDM UWB y WiMAX 802.16e en la red PON propuesta para las configuraciones: WiMAX $10 \mathrm{MHz}$ BW para (a) UWB Ch1 y Ch2, y para (b) UWB TFC1.

En cuanto a la transmisión WiMAX, en la Figura 4.23 se muestran los EVM medidos para una transmisión WiMAX $10 \mathrm{MHz} \mathrm{BW}$ ante la presencia de las dos configuraciones MB-OFDM UWB. En la figura, se representa con una línea discontinua el umbral de $-15 \mathrm{~dB}$ EVM para garantizar una comunicación WiMAX basada en IEEE 802.16e con una modulación QPSK 1/2. Como se observa en la figura, el umbral de EVM WiMAX se cumple para configuraciones PON con enlaces de hasta $25 \mathrm{~km}$ SSMF. La presencia de MB-OFDM UWB introduce una degradación de unos $2 \mathrm{~dB}$ EVM en la distribución WiMAX. Concretamente, para un enlace $25 \mathrm{~km}$ SSMF con una potencia óptica entregada de $1 \mathrm{dBm}$, la EVM para una configuración WiMAX $20 \mathrm{MHz}$ BW varía de $-35.45 \mathrm{~dB}$ para la transmisión WiMAX aislada hasta $-33.45 \mathrm{~dB}$ para la distribución simultánea con MB-OFDM UWB con Ch1 y Ch2.

Mediante el estudio experimental presentado en esta sección, se ha demostrado la viabilidad de la distribución simultánea de señales WiMAX 802.16e y MB-OFDM UWB en redes PON de hasta $25 \mathrm{~km}$ SSMF. En este caso particular, la eficiencia espectral de las configuraciones UWB y WiMAX basadas en OFDM es de $0.3788 \mathrm{bit} / \mathrm{s} / \mathrm{Hz}$ y $0.634 \mathrm{bit} / \mathrm{s} / \mathrm{Hz}$, respectivamente. La eficiencia espectral se calcula en este caso cómo el régimen binario de la comunicación en el ancho de banda de la transmisión. Se ha observado cómo la distribución simultánea en una red PON se traduce en una degradación leve de ambos sistemas, alrededor de 3 dB EVM UWB para las configuraciones MB-OFDM UWB y de 2 dB EVM WiMAX para WiMAX 802.16e. Por otro lado, también se ha observado que para valores de potencia óptica por encima de $3 \mathrm{dBm}$, los valores de EVM para UWB y WiMAX se vuelven cuasi constantes. El estudio de los efectos de alta potencia óptica en las fibras SMF y su repercusión en la 
transmisión y distribución de señales inalámbricas de elevado régimen binario, queda fuera del marco de esta Tesis Doctoral. Otro aspecto a considerar dentro de las líneas futuras de esta Tesis Doctoral, es la evaluación de las prestaciones del sistema para mayores regímenes binarios, por ejemplo, en el caso de UWB para modulaciones DCM con regímenes binarios de hasta $480 \mathrm{Mbit} / \mathrm{s}$ y para WiMAX, con modulaciones del tipo 64 QAM. El uso de modulaciones más complejas conlleva una mayor linealidad en los sistemas radio-sobre-fibra como se indica en [Alv09a].

A continuación, se evalúa el uso de otras técnicas que permiten aumentar la eficiencia espectral de la distribución simultánea de UWB y WiMAX sobre redes basadas en sistemas RoSSMF. En concreto, se propone el uso de técnicas de división por polarización (PDM, Polarization Division Multiplexing) en la transmisión óptica. Este tipo de técnicas se utiliza habitualmente para aumentar el régimen binario de las transmisiones sobre redes ópticas, por ejemplo, en [San08], se consiguen $13.4 \mathrm{Tbit} / \mathrm{s}$ (134 x $111 \mathrm{Gbit} / \mathrm{s} / \mathrm{ch})$ sobre $3600 \mathrm{~km}$ de fibra SMF.

\subsubsection{Técnica PDM para la distribución simultánea}

En esta sección, se propone el uso de técnicas de multiplexación en división por polarización (PDM), para aumentar la eficiencia espectral de la distribución simultánea de UWB y WiMAX en un sistema RoSSMF de tipo PON. Estas técnicas también permiten minimizar las interferencias mutuas entre UWB y WiMAX debido a su coexistencia espectral.

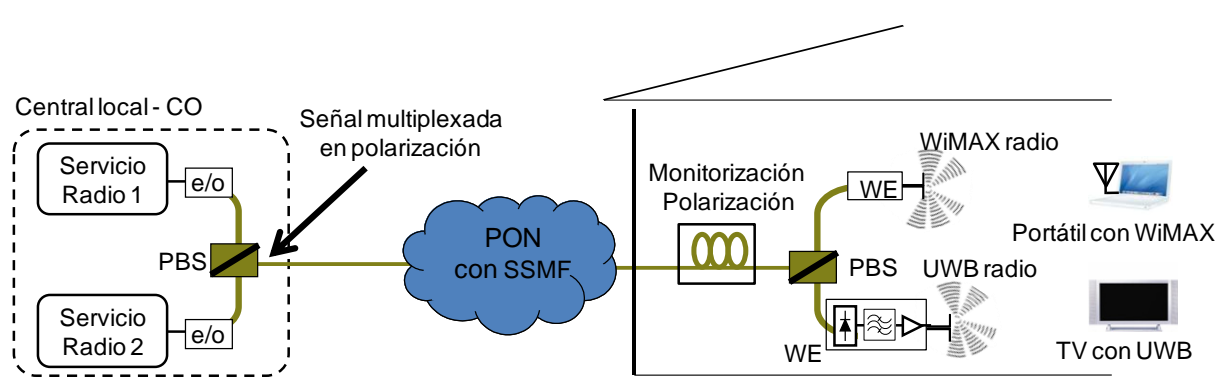

Figura 4.24. Concepto de convergencia inalámbrica de servicios radio WiMAX y

UWB sobre una red PON con RoF mediante técnicas PDM.

En la Figura 4.24, se presenta el concepto asociado a la técnica de PDM propuesta en esta sección. En esta figura, la CO genera señales radio bajo los estándares UWB y WiMAX, como se indica en los bloques genéricos etiquetados como servicio radio 1 y servicio radio 2. Habitualmente, estas señales se convierten al dominio óptico mediante moduladores externos. Tras el ajuste en polarización de ambas señales, éstas se unen mediante un combinador de haces polarizado (PBS, Polarization Beam Splitter/Combiner), y posteriormente se distribuyen a través de la red óptica de acceso 
de fibra SSMF de tipo PON hacia los usuarios. En el equipamiento del usuario, la señal óptica es reajustada en polarización para que sus componentes polarizadas se maximicen con los ejes lento y rápido del PBS. Este proceso es necesario para poder separar las dos polarizaciones ortogonales. Una vez se ha separado en polarización cada servicio radio, éste se foto-detecta, filtra, amplifica y radia de forma directa hacia los dispositivos o usuarios finales. Esta técnica de PDM permite una mayor eficiencia espectral, puesto que se distribuyen dos polarizaciones por cliente, cada una portando como mínimo una señal radio UWB o WiMAX. Por otro lado, el acceso multiusuario en este tipo de redes puede implementarse mediante técnicas por multiplexación en división por longitudes de onda (WDM) o por subportadoras (SCM) [Cha08b], como ya se comentó en el capítulo 2 de esta Tesis Doctoral.

Las técnicas PDM aplicadas a redes basadas en sistemas RoF, requieren de la monitorización de la polarización de la señal en el receptor. Sin embargo, en la mayoría de resultados experimentales relacionados con esquemas de PDM, como en [Jen08] y [Sch08], no se hace mención a los aspectos de la monitorización automática de la polarización. Por ello, en esta sección, tampoco se contemplan las técnicas actuales de monitorización automática de la polarización óptica en tiempo real.

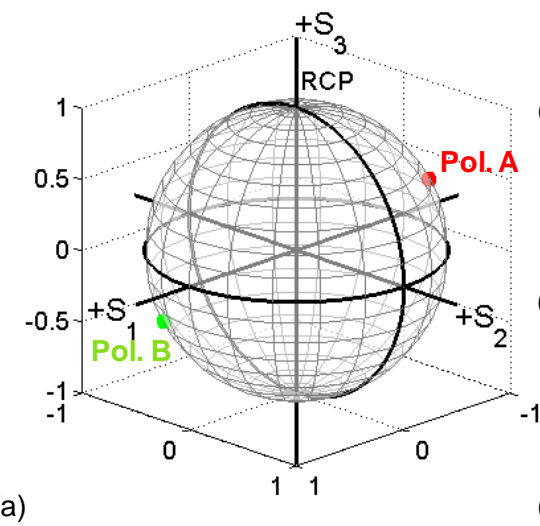

(b)

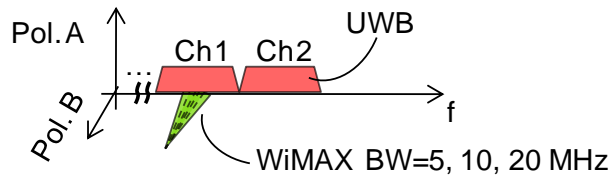

(c)

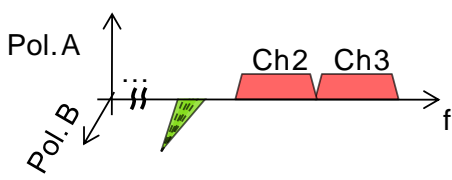

(d)

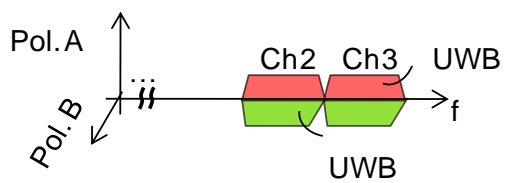

Figura 4.25. (a) Representación de las polarizaciones ortogonales de la señal multiplexada en polarización (PM) entregada a la red PON. Esquemas de multiplexación por división en polarización de tipo (b) cocanal, (c) de canal adyacente y (d) UWB dual multiplexado.

La evaluación de PDM para la distribución conjunta de UWB y WiMAX en redes basadas en sistemas RoF PON se ha analizado conforme a tres diferentes estrategias de polarización, como se observa en la Figura 4.25 (b), (c) y (d). En la Figura 4.25 (b) se presenta la estrategia de PDM denominada polarización cocanal. En ella, la señal UWB está localizada en las subbandas 1\# y 2\# de MB-OFDM UWB para [ECMA368], 
denominadas Ch1 y Ch2, sobre una polarización denominada Pol. A. La señal WiMAX basada en IEEE 802.16e, se genera con una frecuencia central de $3.5 \mathrm{GHz}$ y se distribuye bajo una polarización Pol. B ortogonal a Pol. A. La ortogonalidad de ambas polarizaciones se observa en la Figura 4.25 (a), donde se muestra el estado de polarización asociado a cada una de las ramas de la señal óptica en CO antes del combinador PBS de la Figura 4.24. Desde el punto de vista de la transmisión óptica, esta configuración es la más restrictiva, puesto que WiMAX y UWB se superponen espectralmente, aunque se transmiten en base a polarizaciones ortogonales.

La segunda estrategia PDM es la denominada polarización de canal adyacente, como se observa en la Figura 4.25 (c). Esta propuesta se basa en la transmisión de la señal UWB en las subbandas 2\# y 3\# MB-OFDM UWB con Pol. A, y la transmisión de la señal WiMAX 802.16e centrada en $3.5 \mathrm{GHz}$ mediante Pol. B. Esta estrategia evita la coexistencia espectral en banda para reducir la interferencia mutua entre WiMAX y UWB. En el caso de las estrategias PDM por polarización cocanal y por polarización de canal adyacente, se generan señales WiMAX con anchos de banda nominales de $5 \mathrm{MHz}, 10 \mathrm{MHz}$ y $20 \mathrm{MHz}$ para su evaluación.

Por último, se evalúa la transmisión de UWB en ambas polarizaciones, es decir, mediante técnicas de multiplexado en polarización, como se observa en la Figura 4.25 (d). Esta estrategia PDM permite doblar la eficiencia espectral de la transmisión MB-OFDM UWB sobre RoF PON.

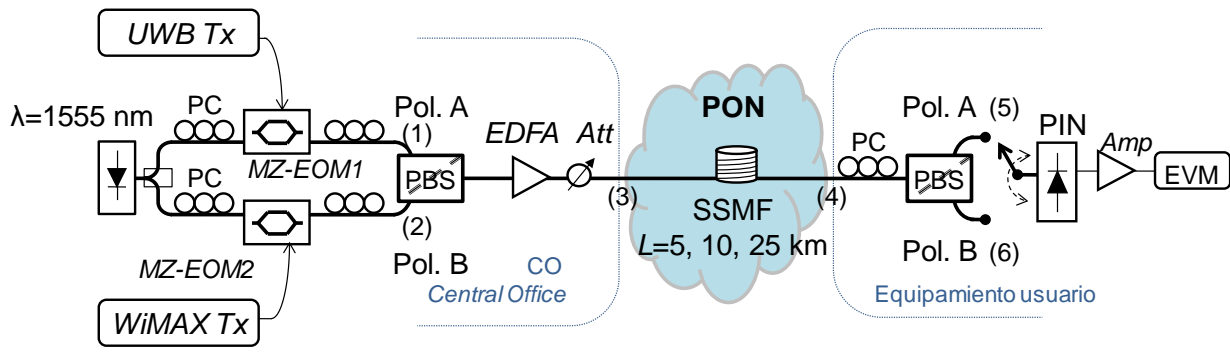

Figura 4.26. Esquema del montaje experimental para la evaluación de la distribución simultánea de señales UWB y WiMAX en sistemas RoF PON mediante distintas estrategias PDM.

La Figura 4.26 muestra el montaje experimental realizado para evaluar la distribución conjunta de MB-OFDM UWB y WiMAX 802.16e en sistemas RoF mediante las tres estrategias PDM comentadas anteriormente. El montaje se divide en tres secciones.

La primera sección, o CO, se corresponde con la generación de la señal óptica a transmitir en RoSSMF PON. Se observan dos bloques denominados UWB Tx y 
WiMAX Tx, que generan las señales radio basadas en MB-OFDM UWB y WiMAX IEEE 802.16e, respectivamente. Estas señales radio son moduladas electro-ópticamente en un modulador externo Mach-Zehnder trabajando en cuadratura QB, denominados MZ-EOM en la Figura 4.26. Cabe destacar que la señal portadora óptica es generada mediante un láser ECL con portadora óptica en $\lambda=1550 \mathrm{~nm}$. Tras la conversión E/O de las señales WiMAX y UWB, se realiza un ajuste de la polarización asociada de cada señal óptica mediante un PC. Por un lado, se reajusta la polarización asociada a la señal óptica en el punto (1) del esquema al estado de polarización lineal horizontal, que en adelante se denomina Pol. A. Por otro lado, en el punto (2) del esquema, se realiza un ajuste de la polarización al estado de polarización lineal vertical, en adelante Pol. B. Estos ajustes permiten que las señales ópticas presenten polarizaciones ortogonales entre sí, como se muestra en la Figura 4.25 (a). Una vez conformadas en polarización las señales ópticas, éstas se combinan en un PBS, obteniendo la señal óptica multiplexada en polarización (PM, Polarization-Multiplexed). La señal PM es amplificada ópticamente en un EDFA Amonics 30-B-FA. Finalmente, la CO entrega esta señal óptica PM amplificada para su distribución sobre los diferentes enlaces SSMF de $5 \mathrm{~km}, 10 \mathrm{~km}$ y $25 \mathrm{~km}$. Cabe destacar que el EDFA mantiene una potencia de salida constante para no incrementar el ruido introducido a la red PON. La potencia óptica total entregada a la red PON en el punto (3) de la Figura 4.26 se ajusta a un rango de potencias de valores $\{-3,-1,1,3,5,7\} \mathrm{dBm}$. Esto se consigue mediante la utilización de un atenuador óptico variable, que permite evaluar el comportamiento de la señal PM desde el punto de vista de la señal recibida en el cliente. El uso de un EDFA en la sección $\mathrm{CO}$ es compatible con la definición de una red PON, como se observa en la revisión de infraestructuras FTTH PON mostrada en [Lee06].

Tras la transmisión óptica a través de los enlaces SSMF que conforman la red PON, la señal PM se entrega a la sección del equipamiento del usuario, en el punto (4) de la Figura 4.26. La polarización de la señal óptica PM se ajusta manualmente mediante un PC, tras el cual un divisor de polarización PBS permitir obtener la señal óptica con Pol. A y con Pol. B en los puntos (5) y (6) del esquema. Tras el PBS, estas señales son foto-detectadas mediante un PIN de $0.7 \mathrm{~A} / \mathrm{W}$ de responsividad. En este montaje, la señal foto-detectada es reamplificada por un LNA, para poder analizar la calidad de la transmisión mediante el estudio del EVM asociado a cada señal radio UWB y WiMAX. Para ello, se utiliza un DSA Agilent 80000B con un software de procesado de señal.

En este punto, es necesario comentar la complejidad que supone el control manual de la polarización en el montaje, que podría ser reemplazado en un sistema real por un estabilizador automático de la polarización como el expuesto en [Yan04]. En la evaluación experimental realizada en esta sección, la monitorización de los estados de polarización (SOP, State-of-Polarization) de la señal óptica en los puntos (1), (2), (5) y (6) de la Figura 4.26, se ha realizado gracias a un analizador óptico de la polarización 
intermedia in-line Optellios PS2300B y a los diferentes ajustes manuales de los correspondientes PC.

A continuación, tras introducir los dispositivos y las funcionalidades de este montaje, se analiza la configuración PDM cocanal para WiMAX y UWB.

\subsubsection{PDM cocanal}

En este caso, se evalúa la distribución simultánea de MB-OFDM UWB y WiMAX 802.16e en un sistema RoSSMF de tipo PON con una estrategia PDM cocanal. Para ello se sigue la estrategia de polarización ortogonal Pol. A y Pol. B mostrada en la Figura 4.25 (b).

En el montaje experimental de la Figura 4.26, los transmisores UWB y WiMAX se configuran para implementar el caso de coexistencia espectral asociado a la estrategia PDM cocanal. El UWB Tx está compuesto por dos módulos DV9110, que generan señales MB-OFDM UWB según [ECMA368], implementando UWB Ch1 en la subbanda 1\# y UWB Ch2 en la subbanda 2\#. Los canales Ch1 y Ch2 se implementan con TFC5 y TFC6, respectivamente, y proporcionan regímenes binarios de $200 \mathrm{Mbit} / \mathrm{s}$, lo que permite una comunicación UWB de $400 \mathrm{Mbit} / \mathrm{s}$ de régimen binario agregado. Los principales parámetros de la señal UWB se resumen en la Tabla 4.3.

El WiMAX Tx de la Figura 4.26 implementa una señal WiMAX basada en IEEE 802.16e, la cual se sintetiza mediante software y se genera mediante un generador de señales vectoriales, Agilent ESG 4438C. Esta señal WiMAX 802.16e se centra en $3.5 \mathrm{GHz}$ y contempla anchos de banda nominales de $5 \mathrm{MHz}, 10 \mathrm{MHz}$ y $20 \mathrm{MHz}$. La Tabla 4.6 resume los principales parámetros de la señal WiMAX generada.
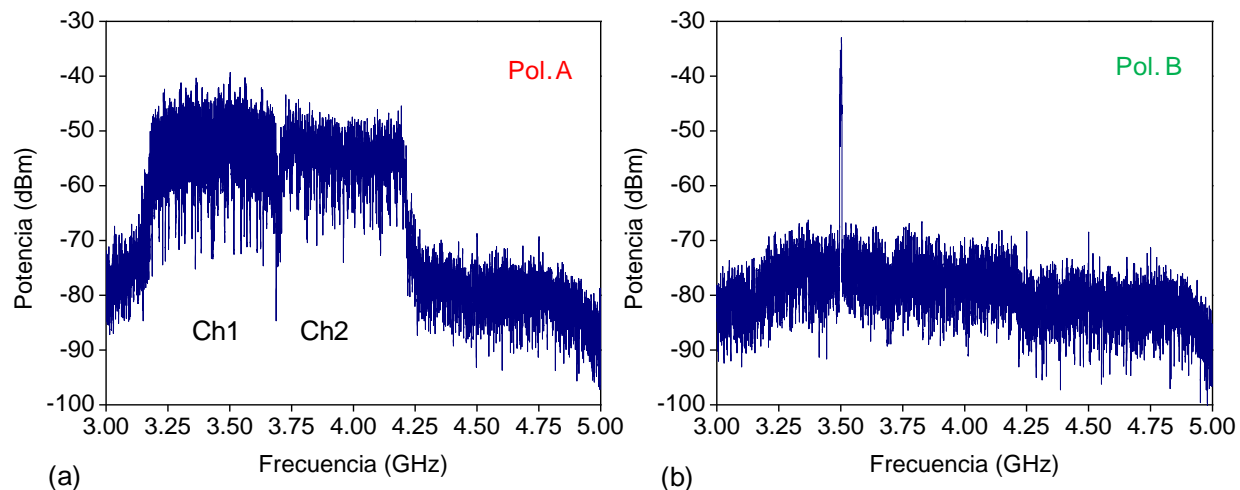

Figura 4.27. Densidad espectral de potencia de las señales MB-OFDM UWB y WiMAX 802.16e recibidas tras un enlace $25 \mathrm{~km}$ SSMF en la configuración PDM cocanal para (a) Pol. A y (b) Pol. B. RBW = 1 MHz. 
La Figura 4.27 muestra el espectro de la señal PM recibida tras su distribución óptica en un enlace de $25 \mathrm{~km}$ SSMF para la estrategia PDM cocanal. En la Figura 4.27 (a) se muestra el espectro asociado para Pol. A tras foto-detectar en el punto (5) de la Figura 4.26. En este caso, se observa como sólo están presentes las componentes UWB Ch1 y Ch2. En la Figura 4.27 (b), se muestra el espectro asociado a Pol. B tras foto-detectar en el punto (6) de la Figura 4.26. En la figura, se observa la señal WiMAX $10 \mathrm{MHz}$ BW y cómo se rechazan las señales UWB conformadas según Pol. A. Comparando las señales UWB mostradas en los espectros para Pol. A y Pol. B, se observa claramente un nivel de rechazo cercano a $30 \mathrm{~dB}$ entre la señal deseada y la no deseada tras la discriminación realizada por el PBS. En esta evaluación, la relación de rechazo entre polarizaciones en el PBS se corresponde con la interferencia residual de polarización cruzada entre la señal transmitida con una polarización y la transmitida con una polarización ortogonal a la anterior. Así pues, el nivel de rechazo obtenido entre señales, indica la capacidad del PBS de resolver las diferentes señales UWB y WiMAX, al distribuirlas mediante PDM en el punto (4) y su posterior división en el punto (5) y en el punto (6) de la Figura 4.26.
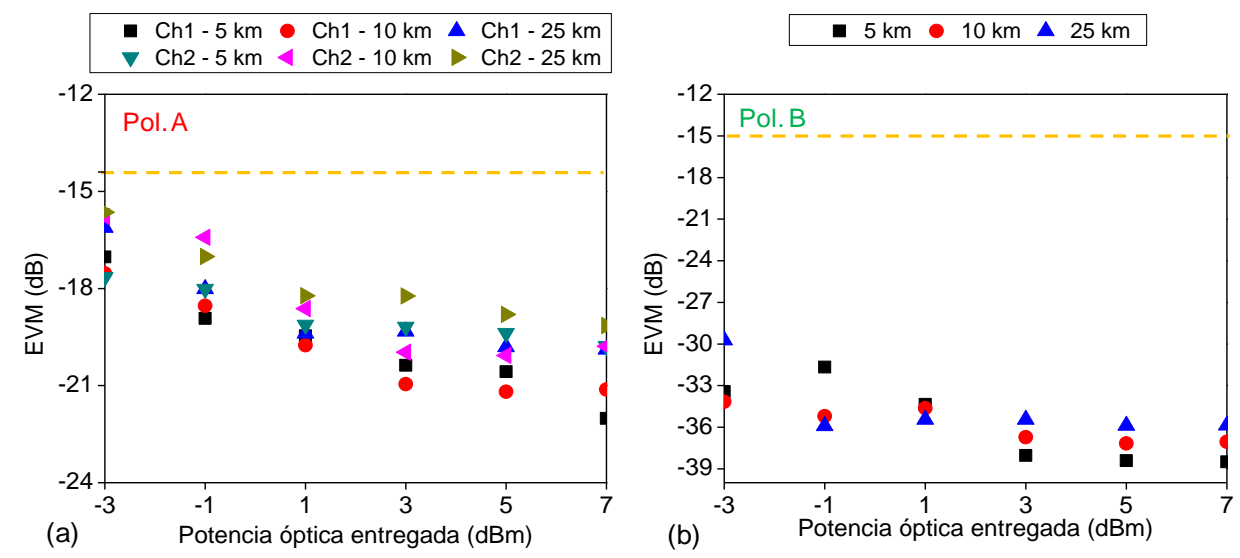

Figura 4.28. EVM medido para las señales (a) UWB Ch1 y Ch2 con Pol. A y (b) WiMAX $5 \mathrm{MHz}$ BW con Pol. B recibidas, según diferentes potencias ópticas entregadas en el sistema RoSSMF PON propuesto.

En la Figura 4.28 se muestra el EVM medido en los puntos (5) y (6) de la Figura 4.26 para las señales MB-OFDM UWB Ch1 y Ch2 en Pol. A y WiMAX 5 MHz BW Pol. B, transmitidas bajo la configuración de PDM cocanal. Los valores EVM UWB mostrados en la Figura 4.28 (a), se sitúan siempre por debajo del umbral EVM UWB de $-14.4 \mathrm{~dB}$, lo que asegura la comunicación. Cabe destacar que el aumento de potencia óptica entregada mejora la calidad del enlace UWB. Con respecto al EVM WiMAX medido, la Figura 4.28 (b) indica la viabilidad de transmisiones WiMAX 5 MHz BW de hasta $25 \mathrm{~km}$ SSMF en base a la estrategia PDM cocanal. De nuevo, los valores EVM 
para WiMAX $5 \mathrm{MHz}$ BW se sitúan por debajo del umbral EVM de -15 dB que asegura la comunicación WiMAX según [IEEE802.16e]. A su vez, el aumento de potencia óptica entregada se traduce en una mejora leve del EVM WiMAX medido.
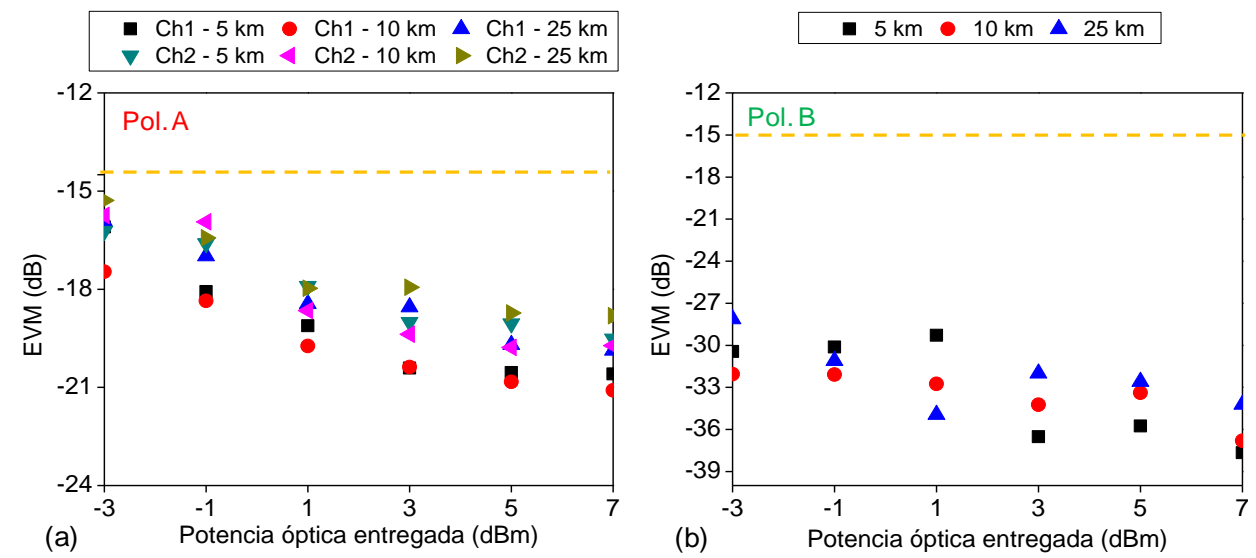

Figura 4.29. EVM medido para las señales (a) UWB Ch1 y Ch2 con Pol. A y (b) WiMAX $10 \mathrm{MHz}$ BW con Pol. B recibidas, según diferentes potencias ópticas entregadas en el sistema RoSSMF PON propuesto.

En la Figura 4.29, se muestra el EVM medido para las señales MB-OFDM UWB Ch1 y Ch2 Pol. A y WiMAX 10 MHz BW Pol. B transmitidas bajo la configuración de PDM cocanal. Los valores EVM UWB mostrados en la Figura 4.29 (a) indican que la comunicación UWB es viable con enlaces PON de hasta $25 \mathrm{~km} \mathrm{SSMF}$, debido a que se sitúan por debajo del umbral EVM UWB. A su vez, el aumento de potencia óptica entregada mejora la calidad del enlace UWB. Con respecto al EVM WiMAX medido, la Figura 4.29 (b) indica que se pueden conseguir transmisiones WiMAX $10 \mathrm{MHz}$ BW de hasta $25 \mathrm{~km}$ SSMF en base a esta estrategia PDM cocanal. El EVM medido para WiMAX $10 \mathrm{MHz}$ BW se localiza por debajo del umbral EVM de $-15 \mathrm{~dB}$, asegurando la comunicación. A su vez, el aumento de potencia óptica entregada se traduce en una mejora leve del EVM WiMAX. 

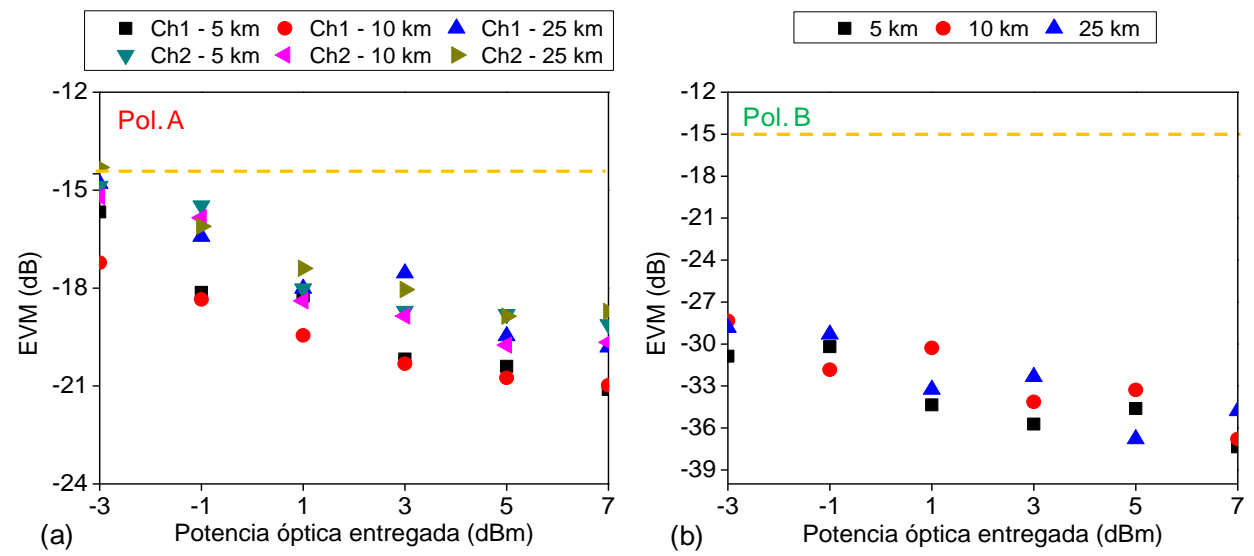

Figura 4.30. EVM medido para las (a) UWB Ch1 y Ch2 con Pol. A y (b) WiMAX $20 \mathrm{MHz}$ BW con Pol. B recibidas según diferentes potencias ópticas entregadas en el sistema RoSSMF PON propuesto.

En la Figura 4.30, se presenta el EVM medido en los puntos (5) y (6) de la Figura 4.26 para las señales MB-OFDM UWB Ch1 y Ch2 Pol. A y WiMAX $20 \mathrm{MHz}$ BW Pol. B transmitidas en base a la configuración de PDM cocanal propuesta. Los valores EVM UWB mostrados en la Figura 4.30 (a) indican que la comunicación UWB es viable para enlaces PON de hasta $25 \mathrm{~km}$, al situarse por debajo del umbral EVM de -14.4 dB. Sin embargo, se observa cómo para bajas potencias de transmisión óptica, el EVM UWB medido para $25 \mathrm{~km}$ SSMF sobrepasa el umbral EVM UWB, comprometiendo la viabilidad de la comunicación UWB. Cabe destacar que el aumento de potencia óptica entregada mejora la calidad del enlace UWB evaluado. Los valores EVM WiMAX mostrados en la Figura 4.30 (b) indican que se pueden conseguir transmisiones WiMAX $20 \mathrm{MHz}$ BW de hasta $25 \mathrm{~km}$ SSMF con esta estrategia PDM cocanal. El EVM medido para WiMAX $20 \mathrm{MHz}$ BW se localiza por debajo del umbral EVM de $-15 \mathrm{~dB}$, asegurando la comunicación. A su vez, el aumento de potencia óptica entregada se traduce en una mejora leve del EVM WiMAX medido.

Comparando las medidas del factor EVM UWB mostradas en la Figura 4.28, Figura 4.29, y Figura 4.30, se observa cómo el aumento del ancho de banda nominal de la señal WiMAX produce una degradación en UWB, especialmente en UWB Ch1. Aunque la degradación sufrida es menor de $1 \mathrm{~dB}$, la transmisión UWB para enlaces de $25 \mathrm{~km}$ SSMF para una potencia entregada de $-3 \mathrm{dBm}$ no está asegurada. Los valores EVM UWB de la Figura 4.28, Figura 4.29, y Figura 4.30, corroboran la presencia de interferencia WiMAX residual tras la discriminación en polarización, aunque no se aprecie en el espectro mostrado en la Figura 4.27. 


\subsubsection{PDM de canal adyacente}

En este caso, se evalúa la distribución simultánea de MB-OFDM UWB y WiMAX 802.16e en un sistema RoSSMF de tipo PON con una estrategia PDM de canal adyacente. Para ello se sigue la estrategia de polarización de la Figura 4.25 (c).

Para esta evaluación, se configura el UWB Tx de la Figura 4.26, formado por dos módulos DV9110, para generar dos canales MB-OFDM UWB centrados en la subbandas 2\# y 3\#. Estos canales se denominan UWB Ch2, centrado en la frecuencia de $3.96 \mathrm{GHz}$, y UWB Ch3, centrado en $4.488 \mathrm{GHz}$. Ambos canales proporcionan un régimen binario de $200 \mathrm{Mbit} / \mathrm{s}$, lo que permite una comunicación UWB de $400 \mathrm{Mbit} / \mathrm{s}$ de régimen binario agregado. Estos canales se ajustan en polarización Pol. A tras su conversión E/O. Los principales parámetros de la señal UWB se resumen en la Tabla 4.3, donde en lugar de Ch1 se configura un canal Ch3 asociado a un código TFC7 para la banda de frecuencias de $4.184 \mathrm{GHz}$ a $4.712 \mathrm{GHz}$.

La señal WiMAX IEEE 802.16e generada por WiMAX Tx se centra en la frecuencia de $3.5 \mathrm{GHz}$, con anchos de banda nominales de $5 \mathrm{MHz}, 10 \mathrm{MHz}$ y $20 \mathrm{MHz}$. Los principales parámetros se resumen en la Tabla 4.6. La señal WiMAX, tras su conversión E/O, se maximiza con un ajuste en polarización de acuerdo a Pol. B, siendo Pol. A y Pol. B ortogonales.

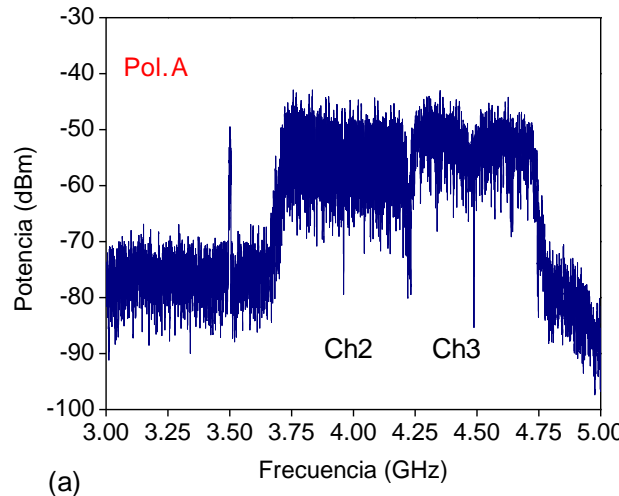

Figura 4.31. Densidad espectral de potencia de las señales MB-OFDM UWB y WiMAX 802.16e recibidas tras un enlace de $25 \mathrm{~km}$ SSMF en la configuración PDM de canal adyacente para (a) Pol. A y (b) Pol. B. RBW = 1 MHz.

La Figura 4.31 muestra el espectro recibido en los puntos (5) y (6) de la Figura 4.26 tras un enlace de $25 \mathrm{~km}$ SSMF. En la Figura 4.31 (a) se muestra el espectro de la señal a la salida del PBS en el punto (5) del esquema correspondiente con Pol. A. En este caso se observa el espectro de la señal MB-OFDMUWB, pero también aparece la interferencia residual de polarización cruzada debido a la distribución conjunta con la 
señal WiMAX Pol. B. La Figura 4.31 (b) muestra la señal correspondiente a Pol. B a la salida del PBS en el punto (6) del esquema, en este caso la señal WiMAX $10 \mathrm{MHz}$ BW. En esta figura, se observa una relación de rechazo de unos $30 \mathrm{~dB}$ con respecto a la señal MB-OFDM UWB Pol. A.
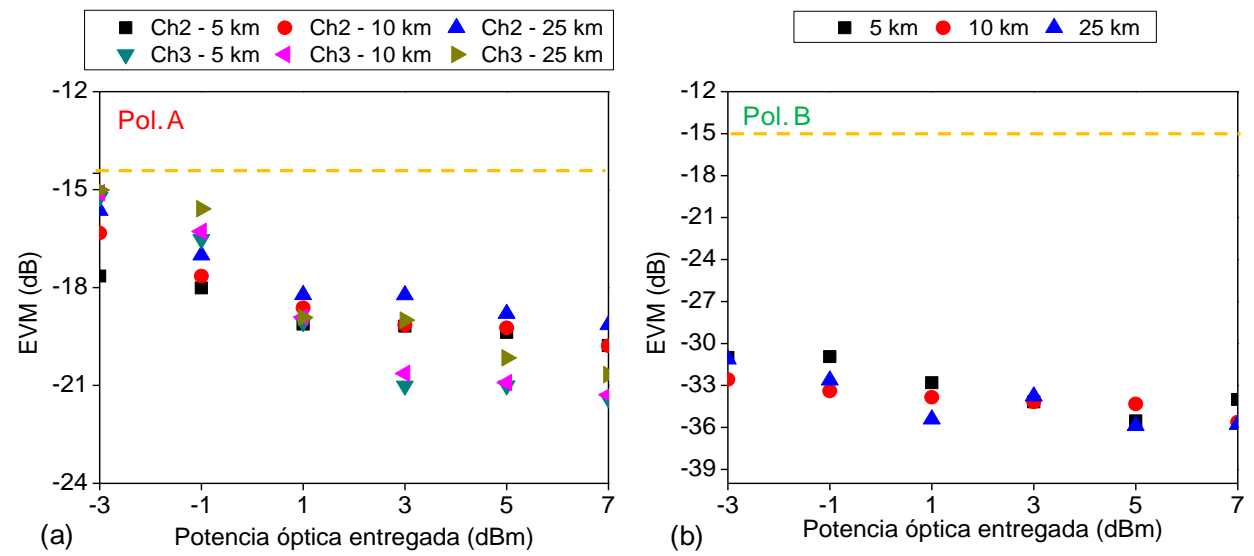

Figura 4.32. EVM medido para las señales (a) UWB Ch2 y Ch3 con Pol. A y (b) WiMAX $10 \mathrm{MHz}$ BW con Pol. B recibidas según diferentes potencias ópticas entregadas en el sistema RoSSMF PON propuesto.

En la Figura 4.32 se presenta el EVM medido para la distribución simultánea de WiMAX $10 \mathrm{MHz}$ BW y MB-OFDM UWB Ch2 y Ch3 mediante PDM de canal adyacente. Los valores EVM UWB mostrados en Figura 4.32 (a), permiten asegurar la comunicación MB-OFDM UWB Ch2 y Ch3 sobre una red PON de hasta $25 \mathrm{~km} \mathrm{SSMF.}$ Sin embargo, se observa cómo para bajas potencias ópticas de transmisión, el EVM UWB medido se sitúa cercano al umbral EVM de $-14.4 \mathrm{~dB}$, por lo que no se puede asegurar la viabilidad de la comunicación UWB. En la Figura 4.32 (b), se muestra el EVM medido para WiMAX $10 \mathrm{MHz}$ BW. En este caso, los valores EVM para WiMAX 802.16e se localizan por debajo del umbral EVM de $-15 \mathrm{~dB}$, asegurando la comunicación WiMAX 802.16e sobre una red PON de hasta 25 km SSMF. 

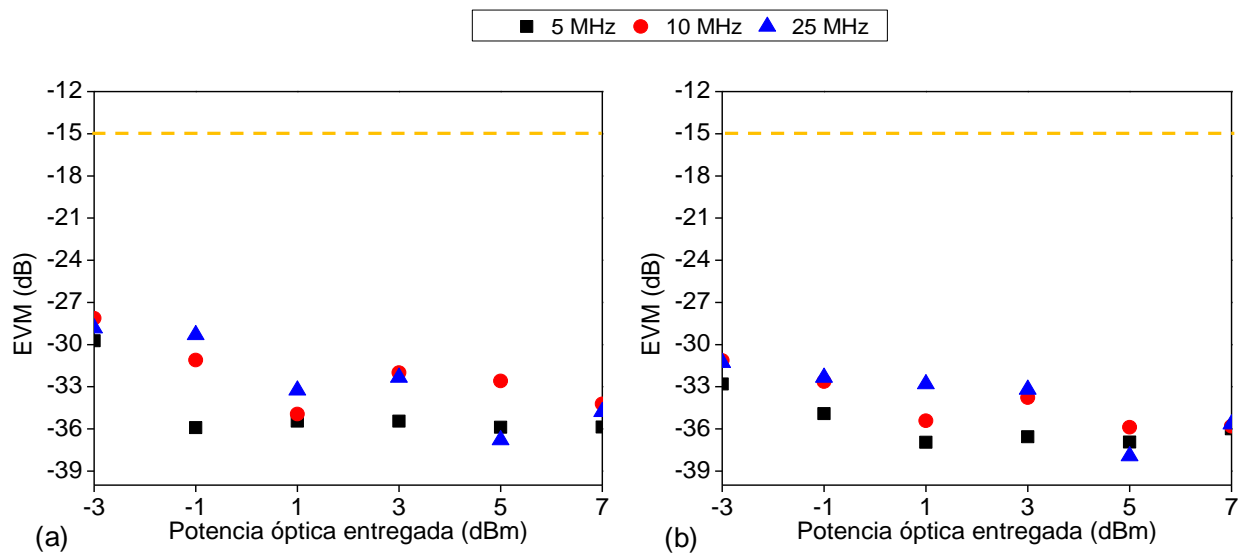

Figura 4.33. EVM medido para las señales recibidas WiMAX tras un enlace de 25 km SSMF según diferentes potencias ópticas entregadas en el sistema RoSSMF PON propuesto para el caso PDM (a) cocanal y (b) adyacente.

A continuación se evalúa el efecto de los diferentes ancho de banda nominales de WiMAX 802.16e para las estrategias PMD cocanal y adyacentes. El EVM medido para las diferentes configuraciones WiMAX según su BW se presentan en la Figura 4.33 (a) para la estrategia PDM cocanal, y en la Figura 4.33 (b) para la estrategia PDM de canal adyacente. Estos valores confirman que la configuración PDM de canal adyacente presenta, para los distintos valores de BW en la señal WiMAX, una mejora de $2 \mathrm{~dB}$ EVM sobre la configuración PDM cocanal. Al mismo tiempo, se observa cómo el aumento de BW se traduce en una degradación del EVM WiMAX en ambas estrategias. Por ejemplo, para una potencia óptica entregada de $-1 \mathrm{dBm}$, el valor EVM para la configuración PDM cocanal WiMAX 5 MHz BW es de $-35.91 \mathrm{~dB}$, mientras que para WiMAX $20 \mathrm{MHz}$ BW es de -29.31 dB. Para la configuración PDM de canal adyacente, para una potencia óptica entregada de $-1 \mathrm{dBm}$, el valor EVM para WiMAX $5 \mathrm{MHz} \mathrm{BW}$ es de $-35.18 \mathrm{~dB}$, mientras que para WiMAX $20 \mathrm{MHz} \mathrm{BW}$ se obtiene un valor de $-32.41 \mathrm{~dB}$ EVM.

Comparando el EVM medido para las estrategias PDM cocanal y de canal adyacente mostrado en la Figura 4.28, Figura 4.29, Figura 4.30 y Figura 4.32, se observa que la transmisión simultánea de MB-OFDM UWB y WiMAX 802.16e sobre enlaces PON de $25 \mathrm{~km}$ SSMF es factible y sólo depende de la potencia óptica entregada en cada caso. Sin embargo, la configuración PDM de canal adyacente presenta un mejor comportamiento que la configuración PDM cocanal en términos de EVM. Esta situación se debe al efecto de la interferencia residual de polarización que aparece en la banda de interés en la estrategia PDM cocanal y fuera de la banda de interés en el caso de PDM de canal adyacente. 


\subsubsection{Prestaciones PDM para la distribución MB-OFDM UWB en modo dual}

A continuación, se evalúa la transmisión dual de señales MB-OFDM UWB en un sistema RoSSMF PON mediante la estrategia PDM exclusivamente sobre señales UWB, con el objetivo de duplicar la eficiencia espectral del sistema. Esta evaluación se basa en la estrategia PDM propuesta en la Figura 4.25 (d).

El montaje experimental se basa en el esquema de la Figura 4.26. En este caso, tanto UWB Tx como WiMAX Tx generan señales MB-OFDM UWB. Cada uno de estos transmisores, está formado por dos módulos DV9110, que generan dos canales MB-OFDM UWB centrados en la subbandas 2\# y 3\#. Estos canales se denominan UWB Ch2, centrado en la frecuencia de $3.96 \mathrm{GHz}$, y UWB Ch3, centrado en 4.488 GHz. Ambos canales proporcionan un régimen binario de $200 \mathrm{Mbit} / \mathrm{s}$, lo que permite una comunicación UWB de $400 \mathrm{Mbit} / \mathrm{s}$ de régimen binario agregado. Los principales parámetros de la señal UWB se resumen en la Tabla 4.3, donde en lugar de Ch1 se configura un canal Ch3 asociado a un código TFC7 para la banda de frecuencias de $4.184 \mathrm{GHz}$ a $4.712 \mathrm{GHz}$. Las señales generadas por cada transmisor, tras su conversión E/O, se ajustan en polarización Pol. A y Pol. B, siendo éstas ortogonales entre sí.

- Ch2 - $5 \mathrm{~km}$ - Ch2 - $10 \mathrm{~km} \Delta \mathrm{Ch} 2-25 \mathrm{~km}$

Ch3 $-5 \mathrm{~km} \bullet \mathrm{Ch} 3-10 \mathrm{~km} \boldsymbol{\Delta}$ Ch3 $-25 \mathrm{~km}$
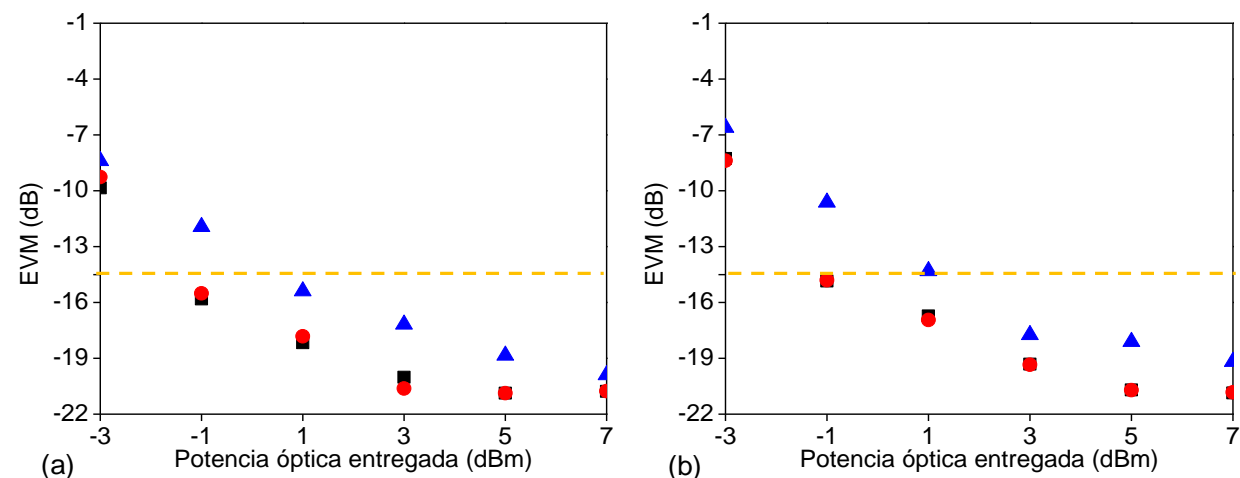

Figura 4.34. EVM medido para una transmisión aislada de señales MB-OFDM UWB (a) Ch2 y (b) Ch3 según Pol. A en el sistema RoSSMF PON propuesto con PDM para diferentes longitudes de enlace SSMF.

En primer lugar, se evalúan las prestaciones del sistema RoSSMF PON cuando sólo uno de los transmisores UWB está activo, según la Pol. A o Pol. B a la cual han sido ajustadas las señales transmitidas. En este caso, la polarización se ajusta a Pol. A.

La Figura 4.34 presenta los valores EVM UWB para una transmisión aislada MB-OFDM UWB Ch2 y Ch3 ajustada a Pol. A en el sistema RoSSMF PON con PDM. 
En ambas figuras se observa como el EVM UWB mejora a medida que aumenta la potencia óptica entregada. Sin embargo, para enlaces de $25 \mathrm{~km}$ SSMF, se necesitan valores de potencia óptica entregada superiores a $1 \mathrm{dBm}$ para asegurar la viabilidad de la comunicación UWB.
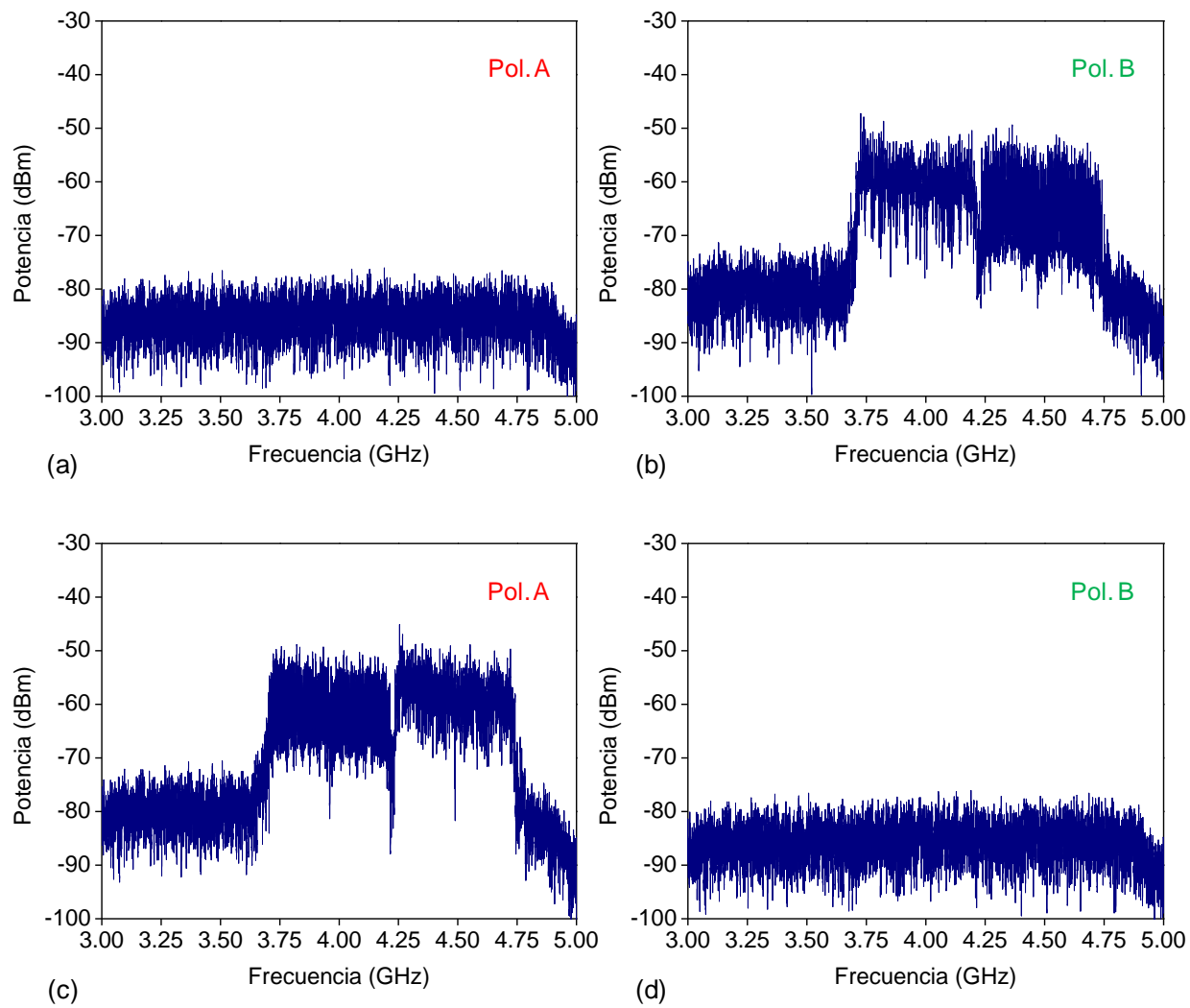

Figura 4.35. Densidad espectral de potencia de las señales MB-OFDM UWB recibida tras $25 \mathrm{~km}$ SSMF en la configuración PDM para transmisión aislada de UWB Ch2 y Ch3 según Pol. A en (a) punto (5) y (b) punto (6) del montaje, y de UWB Ch2 y Ch 3 según Pol. B. en (c) punto (5) y (d) punto (6) del montaje RoSSMF PON propuesto. RBW = 1 MHz.

En la Figura 4.35 se muestran los espectros de MB-OFDM UWB Ch2 y Ch3 recibidos tras una transmisión óptica sobre $25 \mathrm{~km}$ SSMF en los puntos (5) y (6) del montaje descrito en la Figura 4.26. En la Figura 4.35 (c) y (d), se observa cómo la transmisión aislada de MB-OFDM UWB según Pol. A presenta un rechazo en el PBS cercano a los $33 \mathrm{~dB}$. Por otra parte, en la Figura 4.35 (a) y (b), se observa cómo la transmisión aislada de MB-OFDM UWB Ch2 y Ch 3 según Pol. B presenta un nivel de rechazo en el PBS de $30 \mathrm{~dB}$. Estos valores son suficientes para discriminar las señales MB-OFDM UWB en el PBS. 


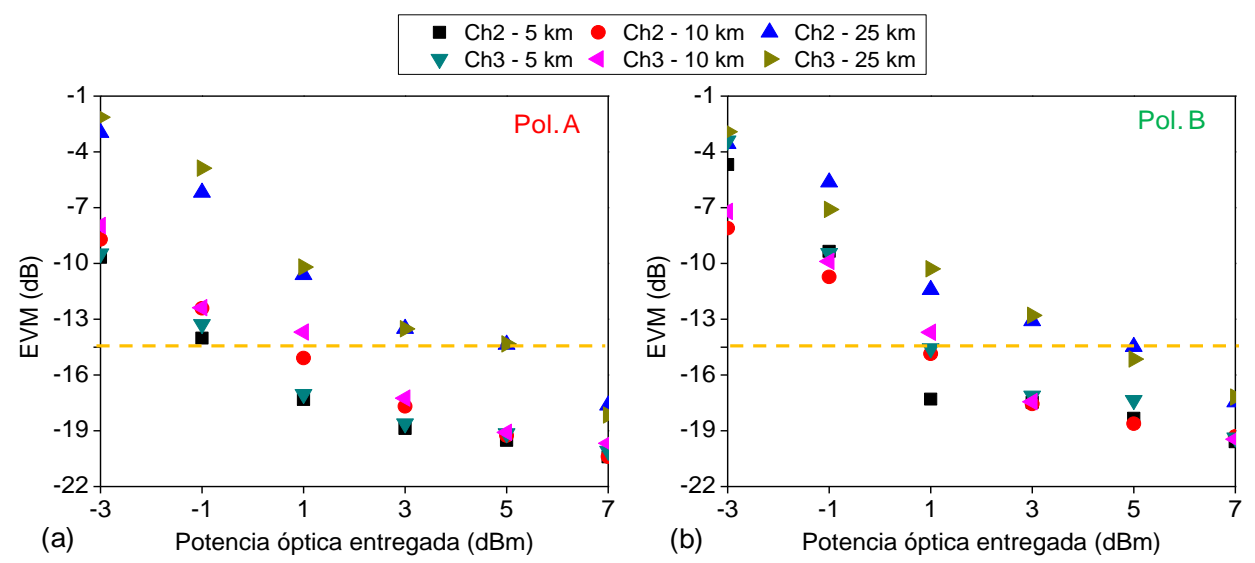

Figura 4.36. EVM UWB medido para las señales MB-OFDM UWB Ch2 y $\mathrm{Ch3}$ (a) Pol. A y (b) Pol. B recibidas para diferentes potencias ópticas entregadas en el sistema RoSSMF PON con PDM propuesto.

En la Figura 4.36 se muestra el EVM medido sobre las señales MB-OFDM UWB recibidas en base a la estrategia PDM en los puntos (5) y (6) de la Figura 4.26 tras los diferentes enlaces SSMF. Estas medidas de EVM UWB muestran cómo el sistema RoSSMF PON con PDM requiere un valor de potencia óptica entregada igual o mayor a $5 \mathrm{dBm}$ para asegurar la viabilidad de la comunicación UWB en enlaces de $25 \mathrm{~km}$ SSMF. Para el resto de enlaces SSMF, el EVM medido se sitúa por debajo del umbral de $-14.4 \mathrm{~dB}$ EVM para potencias ópticas entregadas superiores a $2 \mathrm{dBm}$ y para ambas configuraciones en Pol. A y Pol. B.

Comparando las medidas de EVM UWB presentadas en la Figura 4.34 y Figura 4.36 (a), se observa cómo la estrategia PDM introduce una degradación de aproximadamente $2 \mathrm{~dB}$ EVM. Sin embargo, la estrategia PDM aumenta la eficiencia espectral desde $0.3778 \mathrm{bit} / \mathrm{s} / \mathrm{Hz}$ en una transmisión MB-OFDM UWB Ch2 y $\mathrm{Ch} 3$ aislada, hasta $0.7576 \mathrm{bit} / \mathrm{s} / \mathrm{Hz}$ para una transmisión dual MB-OFDM UWB sobre enlaces de hasta $25 \mathrm{~km}$ SSMF. 
En este capítulo se han evaluado las prestaciones de los sistemas RoF monomodo y multimodo para la distribución simultánea de WiMAX y UWB.

La evaluación experimental de un sistema RoMMF con distribución simultánea de MB-OFDM UWB y WiMAX IEEE 802.16d muestra la limitación en ancho de banda que supone la dispersión modal asociada a las de fibras MMF. El EVM medido para el caso de MB-OFDM UWB muestra una limitación de su alcance máximo radio debido a la transmisión óptica en fibras MMF. En cambio, la señal WiMAX 802.16d presenta una degradación menor y permite comunicaciones WiMAX en distribución conjunta de hasta $300 \mathrm{~m}$ MMF con $30 \mathrm{~m}$ de alcance máximo radio. Las limitaciones del sistema RoMMF vienen impuestas por la dispersión modal asociada a la fibra MMF, que aumenta al trabajar en frecuencias altas, $3.5 \mathrm{GHz}$ para WiMAX y $4.96 \mathrm{GHz}$ para UWB. A su vez, es necesario considerar las limitaciones de ancho de banda que presentan elementos como el VCSEL y el PIN/TIA, los cuales imponen una distorsión que degrada la interoperabilidad de UWB con WiMAX en RoMMF.

Los resultados obtenidos en la evaluación experimental de un sistema RoSSMF para la distribución de señales MB-OFDM UWB, han mostrado un alcance máximo radio de $1 \mathrm{~m}$ para enlaces de $50 \mathrm{~km}$ SSMF. A su vez, se ha demostrado que la transmisión óptica en SSMF de MB-OFDM UWB se penaliza con una reducción del alcance radio máximo. Por ejemplo, se obtiene una penalización de $1 \mathrm{~m}$ de alcance para un enlace de $50 \mathrm{~km}$ SSMF.

Posteriormente, se ha evaluado la distribución conjunta de WiMAX 802.16e y MB-OFDM UWB en un sistema RoSSMF. Para esta evaluación experimental se ha escogido como sistema RoF una red óptica pasiva tipo PON con enlaces de hasta $25 \mathrm{~km}$ SSMF. La distribución simultánea en una red PON con un enlace de $25 \mathrm{~km}$ SSMF introduce una penalización de $3 \mathrm{~dB}$ EVM para la transmisión MB-OFDM UWB y de $2 \mathrm{~dB}$ EVM para WiMAX 802.16e. Se observa cómo el aumento de potencia óptica en transmisión permite una mejora de los valores EVM medidos. Sin embargo, los valores de EVM permanecen constantes para valores superiores a los $3 \mathrm{dBm}$ de potencia óptica. Esta característica se incluye dentro de los efectos producidos por la inyección de señales de alta potencia óptica en las fibras SMF, cuyo estudio queda fuera del marco de esta Tesis Doctoral.

Finalmente se ha evaluado experimentalmente la introducción de técnicas PDM aplicadas a la distribución simultánea de WiMAX 802.16e y MB-OFDM UWB en un sistema RoSSMF de tipo PON. En concreto, se han propuesto dos estrategias de PDM, la primera contempla la interferencia cocanal entre UWB y WiMAX, mientras que la segunda se centra en la interferencia de canal adyacente entre ambos. Comparando los resultados obtenidos para la estrategia PDM cocanal y la estrategia PDM de canal 
adyacente, se observa que la transmisión sobre enlaces PON de $25 \mathrm{~km}$ SSMF es factible en ambas estrategias, y que sólo depende de la potencia óptica entregada en cada caso. Sin embargo, la configuración PDM de canal adyacente presenta un mejor comportamiento que la configuración PDM cocanal en términos de EVM. Esta situación se debe al efecto de la interferencia residual de polarización, situada en la banda de interés en la estrategia PDM cocanal y fuera de la banda de interés en el caso de la PDM de canal adyacente. Por otro lado, el aumento de ancho de banda nominal de la señal WiMAX, produce una mayor degradación en los valores de EVM UWB en la estrategia PDM cocanal que en la estrategia PDM de canal adyacente. Este comportamiento se mantiene cuando se comparan los valores de EVM WiMAX para ambas estrategias.

Finalmente se ha propuesto y demostrado experimentalmente un sistema RoSSMF para la distribución dual de MB-OFDM UWB utilizando PDM en redes ópticas pasivas. El EVM UWB medido muestra una penalización aproximada de 2 dB EVM entre una transmisión MB-OFDM UWB aislada y la transmisión dual PDM. Cabe destacar la viabilidad de la comunicación UWB está asegurada para enlaces de $25 \mathrm{~km} \mathrm{SSMF.} \mathrm{A} \mathrm{su}$ vez, la estrategia PDM aumenta la eficiencia espectral desde $0.3778 \mathrm{bit} / \mathrm{s} / \mathrm{Hz}$ en una transmisión MB-OFDM UWB aislada hasta $0.7576 \mathrm{bit} / \mathrm{s} / \mathrm{Hz}$ para una transmisión dual MB-OFDM UWB sobre enlaces de hasta $25 \mathrm{~km} \mathrm{SSMF.}$

Los resultados obtenidos muestran la efectividad de las estrategias PDM cocanal, PDM de canal adyacente y PDM dual UWB para distancias RoSSMF típicas de sistemas PON, hasta $25 \mathrm{~km}$ SSMF.

Por último, es importante destacar que la linealidad de los elementos clave de los sistemas RoF, como son VCSEL, moduladores externos y foto-detectores, es una de las principales limitaciones encontradas en las evaluaciones experimentales presentadas en este capítulo. A su vez, el efecto de la PMD y la dispersión cromática no se han mostrado como factores limitantes en el análisis experimental realizado de la distribución conjunta de WiMAX y UWB en sistemas RoF. 



\section{Capítulo 5. Linealización del modulador electro-óptico Mach-Zehnder en sistemas radio-sobre-fibra}

Tal y como se ha descrito en el capítulo 4 de esta Tesis Doctoral, la coexistencia inalámbrica en sistemas RoF, es decir, la transmisión de múltiples señales radio sobre una o varias portadoras ópticas, presenta mejores prestaciones cuando la conversión E/O se realiza mediante moduladores externos [Dar08]. El principal factor limitante de las prestaciones de un sistema RoF es la respuesta no-lineal del modulador electro-óptico externo [Cox04]. En el caso de considerar la coexistencia en sistemas RoF de señales radio con características espectrales y márgenes dinámicos muy dispares, es necesario linealizar la respuesta electro-óptica del moduladores.

En este capítulo, se proponen y evalúan técnicas que mejoran la conversión electro-óptica en el modulador tipo Mach-Zehnder (MZM, Mach-Zehnder Modulator). El modulador MZM presenta un comportamiento no-lineal debido a su estructura interferométrica. Esta no-linealidad inherente se traduce en una degradación de la señal óptica modulada por el dispositivo. Esta no-linealidad introduce armónicos no deseados denominados distorsión de intermodulación (IMD, Intermodulation Distorsion).Las IMD más notables son aquellas de segundo y de tercer orden. Se consideran de segundo orden cuando se contemplan las amplitudes de los productos de intermodulación función de la combinación de los fundamentales, siendo A y B fundamentales se considerarían las combinaciones $2 \mathrm{~A}, 2 \mathrm{~B}$ y $\mathrm{A} \pm \mathrm{B}$. Por otra parte se consideran de tercer orden cuando contemplan las amplitudes de los factores de intermodulación 3A, 3B, $2 \mathrm{~A} \pm \mathrm{B}, 2 \mathrm{~B} \pm \mathrm{A}$ y factores de modulación cruzada [Cox04]. Para caracterizar estas IMD no lineales se considera habitualmente al mezclado compuesto de segundo orden (CSO, Composite Second-Order Beat,) y al mezclado compuesto de batidos de tercer orden (CTB, Composite Triple Beat). Estos factores CSO y CTB se definen como el cociente entre los niveles de potencia de los términos de distorsión de segundo o tercer orden y el nivel de potencia eléctrica de la subportadora. 
En un modulador MZM si el ancho de banda de modulación es inferior a una octava, la distorsión de segundo orden o CSO es despreciable, tal y como se describe en [Cox04]. La linealización de un modulador electro-óptico con ancho de banda de modulación menor a una octava, o de banda estrecha, tiene como objetivo reducir la distorsión de tercer orden o CTB, que es la más importante según [Wil97]. En cambio, si trabajamos en anchos de banda multi-octava, por ejemplo con señales UWB que cubren el rango de frecuencias de $3.1 \mathrm{GHz}$ a $10.6 \mathrm{GHz}$, la presencia de no-linealidades tiene un gran impacto y por lo tanto es necesario desarrollar una técnica de linealización que permita reducir tanto el CSO como el CTB.

Este capítulo de la Tesis Doctoral se centra en la evaluación de la linealización del modulador electro-óptico con arquitectura Mach-Zehnder basado en niobato de litio $\left(\mathrm{LiNbO}_{3}\right)$. En primer lugar, se presenta un análisis de las principales características del modulador MZM y de sus parámetros de funcionamiento, así como del comportamiento no-lineal inherente al MZM. A continuación, se revisan las técnicas de linealización eléctricas y técnicas de linealización ópticas. Finalmente se propone, analiza y evalúa experimentalmente una técnica de linealización óptica dual en paralelo para MZM para señales de gran ancho de banda o multi-octava.

\subsection{Modelo básico de un modulador electro-óptico Mach-Zehnder}

El modulador externo más comúnmente usado en la generación óptica de las redes ópticas de acceso que utilizan RoF es el modulador de intensidad basado en la estructura interferométrica Mach-Zehnder. Su principio de funcionamiento es el efecto electro-óptico que modifica el índice de refracción de un medio de forma proporcional al campo aplicado [Bur99]. Este efecto es lineal y propio de cristales anisótropos que no posean simetría de inversión, como el niobato de litio $\left(\mathrm{LiNbO}_{3}\right)$, el arseniuro de galio (GaAs) o el fosforo de indio (InP).

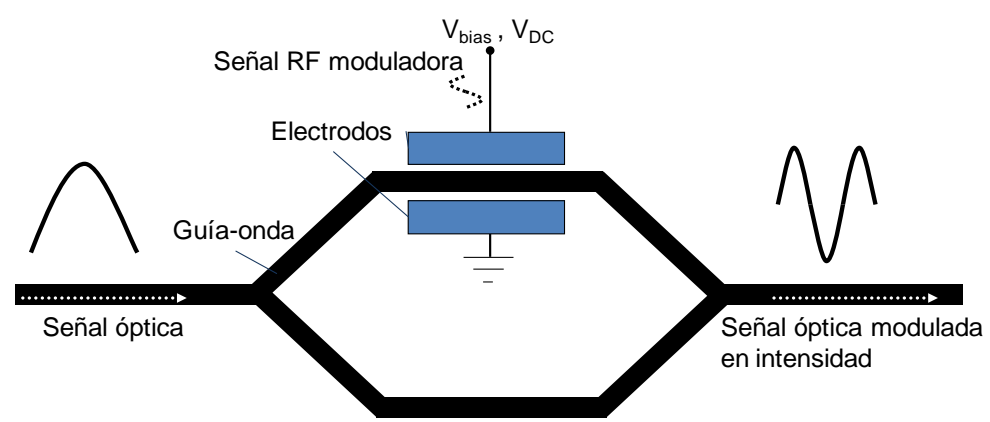

Figura 5.1. Esquema del modulador electro-óptico de intensidad basado en interferómetro Mach-Zehnder. 
La estructura interferométrica Mach-Zehnder se muestra en la Figura 5.1. En esta estructura se observa el uso de una guía-onda para conducir la señal óptica y su división en una estructura interferométrica de dos brazos. En uno de los brazos se aplica una tensión externa a través de unos electrodos que permite, mediante el efecto electro-óptico, variar el índice de refracción del material en el MZM, lo cual se traduce en un cambio de la fase de la señal óptica en ese brazo. La relación entre el efecto electro-óptico en el material y la modulación de intensidad en la señal óptica se analiza en la sección 5.1.2. A continuación se analizan los parámetros característicos de un MZM.

\subsubsection{Parámetros característicos}

Los parámetros característicos de los moduladores MZM pueden definirse a nivel funcional, es decir, relacionados tanto con parámetros de trabajo, como son el rango de longitudes ópticas de operación, el ancho de banda, las pérdidas de inserción y la tensión de media onda $V_{\pi}$, como con parámetros constructivos como es su índice de refracción extraordinario $\left(n_{e}\right)$ o la longitud de los electrodos $L$. Desde el punto de vista de los sistemas RoF los parámetros más importantes son:

(i) Ancho de banda o frecuencia máxima de trabajo, $f_{\max }$

$$
f_{\max } \approx \frac{1}{T_{w}}=\frac{c}{L}\left(n_{m}-n_{o}\right)
$$

El ancho de banda depende de la longitud de los electrodos $L$, mostrados en la Figura 5.1, el índice del modo guiado de microondas $n_{m}$ y el índice del modo guiado óptico en la onda progresiva $n_{o}$. Estos índices son parámetros constructivos que permiten obtener una frecuencia máxima de trabajo cuando están bien adaptados, lo cual se consigue variando parámetros de construcción del MZM como se propone en [Mit95]. Otra posibilidad de aumentar la frecuencia máxima de trabajo es acortar la longitud de los electrodos, pero este procedimiento requiere tensiones de polarización mayores.

(ii) Índice de modulación, $m_{i}$

Este índice indica la profundidad de modulación en la señal óptica según la señal eléctrica moduladora entregada. Para asegurar un correcto funcionamiento del MZM, es conveniente disponer de un índice de modulación alto. El índice de modulación se suele expresar como función de la tensión de operación del modulador, también denominada $V_{\pi}$, puesto que indica la tensión necesaria que se debe aplicar a los electrodos para obtener un cambio de fase $\pi$ [Cor01]. 


$$
m_{i} \approx \frac{\pi}{V_{\pi}}, \quad V_{\pi}=\frac{\lambda g}{n_{e}^{3} r_{33} \alpha_{s o l} L}
$$

La tensión de media onda $V_{\pi}$ depende, como se observa en la ecuación (5.2), de varios parámetros constructivos. Entre estos parámetros están aquellos que hacen referencia a la asimetría del cristal, como son el índice de refracción extraordinario $n_{e} \mathrm{y}$ el tensor de aplicación del campo eléctrico a lo largo del eje de simetría del cristal $r_{33}$. En esta ecuación, $g$ es la separación entre los electrodos de longitud $L$ y $\alpha_{s o l}$ es un factor de solapamiento entre el campo eléctrico aplicado y el campo óptico. Este factor indica la eficiencia de la modulación de la señal, puesto que muestra el espacio físico o la duración temporal durante el cual interactúan ambas señales en los electrodos y guía del modulador MZM. A su vez, $\lambda$ es la longitud de onda óptica de la señal en la guía-onda.

A partir de la ecuación anterior, se puede definir el cambio de fase $(\Delta \phi)$ inducido electro-ópticamente sobre la señal óptica mediante unos electrodos de longitud $L$. Este cambio de fase viene dado por la integral del vector de onda a lo largo de los electrodos, suponiendo que se aplica la misma tensión a lo largo de todo el electrodo tal y como se muestra en la ecuación (5.3).

$$
\Delta \phi=-\pi \frac{n_{e}^{3} r_{33} \alpha_{s o l} L}{\lambda_{0} g} V \rightarrow \Delta \phi=-\pi \frac{V}{V_{\pi}}
$$

Una posible solución para aumentar el índice de modulación, consiste en alargar la longitud de los electrodos $(L)$, lo cual se traduce en una reducción del ancho de banda. Otra posibilidad es disminuir el valor de $V_{\pi}$, para lo cual es necesario actuar sobre el valor de la integral de solapamiento $\left(\alpha_{\text {sol }}\right)$. En este caso, la solución corresponde al punto de mayor eficiencia para la interacción electro-óptica. Esto nos permite obtener un índice de modulación alto manteniendo un ancho de banda adecuado.

Es conveniente destacar que el índice de modulación depende de la excursión máxima de la señal, que viene dada por la tensión aplicada y la tensión de media onda del dispositivo. Además, existen efectos de deriva tanto de la tensión de media onda como de la longitud de onda de trabajo. La variación temporal de la tensión de media onda introduce una submodulación de la señal de RF que limita el margen dinámico de trabajo del modulador. Por otro lado, en [Kel01], se estudia la deriva de la longitud de onda de trabajo en la respuesta del modulador y por tanto, en el índice de modulación. Esta deriva introduce distorsión armónica de segundo orden (IMD2, Second Order Intermodulation Distortion) al no trabajar el modulador en la zona lineal. 
(iii) Pérdidas de inserción

Las pérdidas de inserción se deben al acople de los modos de la señal óptica entrante con la estructura de guía onda. Estas pérdidas se localizan mayoritariamente en la interfaz entre la fibra óptica y el substrato de guía onda. La mejora en las técnicas de acoplo guía-fibra permitirá la reducción de las pérdidas del MZM. Ejemplos de técnicas de acoplo guía-fibra óptica tenemos en [Roe08] donde se proponen un acople basado en estructuras difractivas de tipo grating, que muestran un $55 \%$ de eficiencia de forma experimental y hasta un $80 \%$ deforma teórica.

\subsubsection{Efecto electro-óptico}

El comportamiento electro-óptico del MZM se expresa en función del campo eléctrico a la entrada y los desfases inducidos por la tensión aplicada a los electrodos como consecuencia del efecto electro-óptico. La función de campo eléctrico a la entrada del modulador se escribe como:

$$
E_{i n_{-} m z m}=E_{i n} e^{j \theta_{i}} .
$$

En el caso de un MZM donde sólo se polariza un brazo, o configuración push-pull, que se corresponde con la estructura de la Figura 5.1, la función de campo eléctrico a la salida del mismo se escribe como:

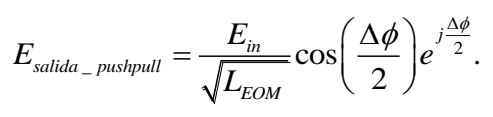

Para un MZM de doble brazo polarizado o dual-drive, en el cual los desfases introducidos por cada brazo están definidos como $\Delta \phi_{1}$ y $\Delta \phi_{2}$, la función de campo eléctrico de salida se escribiría como:

$$
E_{\text {salida }- \text { dual-drive }}=\frac{E_{\text {in }}}{\sqrt{L_{E O M}}} \cos \left(\frac{\Delta \phi_{1}-\Delta \phi_{2}}{2}\right) e^{j \frac{\Delta \phi_{1}+\Delta \phi_{2}}{2}} .
$$

En las ecuaciones (5.5) y (5.6), $L_{E O M}$ es el factor que engloba las pérdidas de potencia tanto en los conectores de E/S como por atenuación óptica de la señal en la guía-onda. Con respecto a los desfases introducidos por cada brazo en el MZM, el parámetro chirp define la modulación residual de fase añadida a la modulación de intensidad, como se muestra en la ecuación (5.7). 


$$
\alpha_{\text {chirp }}=\operatorname{Im}\left[E_{\text {out }}^{*}(t) \frac{d E_{\text {out }}(t)}{d t}\right] / \operatorname{Re}\left[E_{\text {out }}^{*}(t) \frac{d E_{\text {out }}(t)}{d t}\right] \rightarrow \frac{\Delta \phi_{1}+\Delta \phi_{2}}{\Delta \phi_{1}-\Delta \phi_{2}} .
$$

A partir de la ecuación de campo (5.6), la potencia a la salida del MZM se puede expresar en función de la tensión de polarización del modulador, como se muestra en la ecuación (5.8), donde $V_{p o l}$ es la tensión de polarización aplicada, $V_{\pi}(D C)$ es la tensión para un desfase $\pi$ y $P_{I N}$ es la potencia a la entrada del MZM.

$$
P_{\text {out }}=\frac{P_{\text {in }}}{2 \cdot L_{E O M}}\left[1+\cos \left(\frac{\pi V_{p o l}}{V_{\pi}(D C)}\right)\right] \text {. }
$$

En la Figura 5.2, se representa la función de conversión de tensión eléctrica a potencia óptica de un MZM conforme a la ecuación (5.8). En ella se definen los puntos o regiones de trabajo de un MZM. La zona de cuadratura (QB, Quadrature BIAS) es la zona de desfase $\pi / 2$ que se corresponde con la zona de funcionamiento lineal del modulador. En esta función de conversión se distinguen dos zonas extremas de funcionamiento, la de máxima transmisión (MATB, Maximum Transmission Bias) y la de mínima transmisión (MITB, Minimum Transmission Bias), ambas correspondientes con desfases de 0 y $\pi$ radianes entre las ramas del MZM. En la zona MATB se consigue la máxima potencia de salida, mientras que la potencia mínima se consigue en la zona MITB. Estas zonas de trabajo se representan en la Figura 5.2 sobre la curva de polarización. En la figura mostrada QB se corresponde con valores de tensión de polarización $V_{\pi} / 2$.

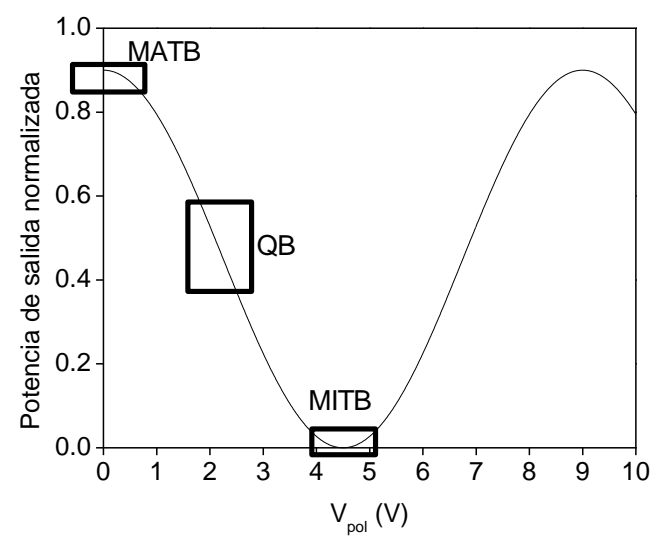

Figura 5.2. Función de transferencia óptica para un MZM con $V_{\pi}(D C)=4.5 \mathrm{~V}$ con pérdidas. 
A continuación se muestra el comportamiento del MZM ante una señal moduladora RF, según el desarrollo matemático de un MZM expuesto en [Cor01]. La señal moduladora de RF se expresa en este caso como:

$$
V=V_{B}+V_{a} \sin \left(\omega_{a} t\right)+V_{b} \sin \left(\omega_{b} t\right)
$$

donde $V_{B}$ es la tensión eléctrica continua o de BIAS y $V_{a}$ y $V_{b}$ son las amplitudes de las señales de RF con frecuencias $\omega_{a} \mathrm{y} \omega_{b}$, respectivamente.

Teniendo en cuenta la siguiente relación entre las funciones Bessel y las funciones trigonométricas:

$$
\cos [\alpha \sin (\beta)]=J_{0}(\alpha)+2 \sum_{k=1}^{\infty} J_{2 k}(\alpha) \cos (2 k \beta)
$$

y a partir de la función de campo de salida del MZM mostrada en la ecuación (5.6), se obtiene la ecuación (5.11) de campo a la salida del MZM para la señal RF moduladora:

$$
E_{\text {out_MZM }}(t)=\frac{E_{\text {in }}}{\sqrt{L}} \cdot \sum_{n=-\infty}^{\infty} J_{n}\left(m_{R F}\right) \cos \left(\phi_{v}+n \phi_{d}\right) e^{j m\left(\omega_{R F} t+\phi_{m}\right)} .
$$

En esta ecuación se observan los desfases relacionados con la señal moduladora, los desfases introducidos en cada rama del MZM, así como aquello relacionados con propiedades intrínsecas de la construcción del modulador, como se observa en [Cor01]. A su vez, la ecuación (5.11) proporciona el valor del índice de modulación de la señal óptica modula en intensidad por una señal RF:

$$
m_{R F}=\frac{\pi V_{R F}}{V_{\pi}}
$$

La ecuación (5.11) permite definir la respuesta electro-óptica del modulador como $T(V)$. Esta respuesta, dada por la ecuación (5.13), presenta los coeficientes de intermodulación $\left(c_{n}\right)$ que aparecen como respuesta a la señal RF moduladora. El desarrollo de estos coeficientes, permiten obtener las diferentes contribuciones a la distorsión en el MZM.

$$
\begin{aligned}
& T(V)=\left.\sum_{n=0}^{\infty} \frac{\left(V-V_{B}\right)^{n}}{n !} \frac{d^{n} T}{d V^{n}}\right|_{V=V_{B}}=c_{0}+\sum_{n=1}^{\infty} c_{n}\left(V-V_{B}\right)^{n}, \\
& \text { donde } \quad c_{0}=T\left(V_{B}\right) \quad y \quad c_{n}=\left.\frac{1}{n !} \frac{d^{n} T}{d V^{n}}\right|_{V=V_{B}} .
\end{aligned}
$$


La modulación de una señal de RF en un modulador MZM introduce productos de intermodulación que distorsionan la señal óptica modulada. Esta distorsión afecta al margen dinámico del modulador y también al margen de funcionamiento libre de espúreos (SFDR, Spurious-Free Dynamic Range) de los enlaces ópticos sobre los cuales funciona este MZM [Cha04]. Para evaluar la contribución de cada producto de intermodulación en la señal óptica a la salida del modulador, se detecta con un fotodiodo de responsividad $R$ como se muestra en la ecuación (5.14).

$$
i_{P D}(t)=\frac{\mathfrak{R} E_{M Z M} E_{M Z M}^{*}}{2}=\frac{\mathfrak{R} P_{p o t \_m z m}}{L}\left(I_{D C}+\sum_{p=1}^{\infty} I_{p R F}(p)\right) .
$$

Desarrollando la ecuación anterior en funciones de Bessel, se obtienen las expresiones correspondientes a la señal continua $I_{D C}$ :

$$
I_{D C}=\sum_{n=-\infty}^{\infty} J_{n}^{2}\left(m_{R F}\right)\left(\cos \left(\phi_{v}+n \phi_{d}\right)\right)^{2}
$$

a los componentes armónicos de tipo par de la señal modulada:

$$
\begin{aligned}
& I_{p R F}(p)=\cos \left(p \omega_{R F}\left(t+\phi_{1}\right)+p \phi_{m}\right) . \\
& {\left[J_{p / 2}^{2}\left(m_{R F}\right)+2(-1)^{p / 2} \sum_{n=(p / 2)+1}^{\infty} J_{n}\left(m_{R F}\right) J_{n-p}\left(m_{R F}\right) \cos \left(\frac{\theta_{2}}{2} p(2 n-p) \omega_{R F}^{2}\right)\right],}
\end{aligned}
$$

y a los componentes armónicos de tipo impar:

$$
\begin{aligned}
& I_{p R F}(p)= \pm 2 \cos \left(p \omega_{R F}\left(t+\phi_{1}\right)+p \phi_{m}\right) . \\
& \cdot \sum_{n=(p / 2)+1}^{\infty}(-1)^{n-(p-1) / 2} J_{n}\left(m_{R F}\right) J_{n-p}\left(m_{R F}\right) \cos \left(\frac{\theta_{2}}{2} p(2 n-p) \omega_{R F}^{2}\right) .
\end{aligned}
$$

Los productos de intermodulación y su influencia en el comportamiento del MZM dependen de la zona de trabajo del mismo. Para la zona QB, la contribución del armónico fundamental $\left(\mathrm{H}_{0}\right)$ de la señal de $\mathrm{RF}$ modulada es máxima y no existe contribución de los armónicos pares, por ejemplo IM2. Para las zonas MATB y MITB, la contribución de los armónicos fundamental e impar $\left(\mathrm{H}_{0}, \mathrm{IM} 3\right)$ es mínima, siendo máxima la de los armónicos pares. Por lo tanto, la zona de trabajo QB es la más adecuada para trabajar con moduladores MZM, puesto que elimina la contribución del armónico de segundo orden y de los armónicos pares en general, y sólo permanecen las contribuciones del armónico fundamental $\left(\mathrm{H}_{0}\right)$ y del armónico de tercer orden (IM3, Third Order Intermodulation), cuya potencia es menor que la de los armónicos de segundo orden (IM2, Second Order Intermodulation). 


\subsubsection{Derivas y efectos de segundo orden}

El comportamiento electro-óptico de un modulador no es constante a lo largo de los periodos de trabajo, ni tampoco a lo largo de la vida útil del MZM. El comportamiento del MZM presenta desviaciones o derivas debido a factores ambientales, al uso intensivo del MZM o a imperfecciones en su construcción y encapsulado. Estas derivas se traducen en distorsiones o efectos de segundo orden sobre la señal modulada ópticamente y en pérdidas introducidas por el MZM.

\subsubsection{Deriva de la tensión de polarización}

Un ejemplo del efecto de estas derivas es la influencia del punto de polarización de un MZM sobre la señal modulada. Para trabajar en cualquiera de las tres regiones de funcionamiento (QB, MITB o MATB) del MZM es necesario mantener una tensión de polarización o BIAS en los electrodos del modulador. Este punto de polarización depende también de parámetros constructivos del MZM y de la longitud de onda de trabajo. Por lo tanto, se ve afectado por variaciones lentas de la propia tensión de polarización aplicada, por variaciones de sus parámetros constructivos y por variaciones en la longitud de onda de trabajo, como se indica en [Kel01]. Esta desviación del punto de polarización produce efectos de distorsión por intermodulación que introducen nuevos armónicos que empeoran la SFDR del sistema.

Por otra parte, la temperatura es otro de los factores que introduce una desviación en el punto de polarización del MZM. En [Nag04], este efecto se relaciona también junto con periodos de funcionamientos largos de más de 8000 horas, es decir con derivas temporales en su funcionamiento.

Por tanto, para asegurar un funcionamiento estable del MZM durante largos periodos de trabajo, se requiere un control dinámico de la tensión de polarización o BIAS. Dentro de estos métodos dinámicos, se encuentran los métodos basados en la modulación de la señal de BIAS con frecuencias RF de test analógicas de muy baja frecuencia. El procesado de estas señales tras su modulación permite conocer la variación de la frecuencia de test RF y actuar sobre la tensión de BIAS para corregir su desviación. Por otro lado, también existen dispositivos comerciales para control dinámico de BIAS, como por ejemplo, el MBC-LN para MZM de PHOTLINE Tech. [MBC05], donde se inyecta una tensión de polarización al MZM y se controla que mantenga su zona de trabajo, mediante el uso de frecuencia de test de entre $300 \mathrm{~Hz}$ y $1800 \mathrm{~Hz}$. 


\subsection{Técnicas de linealización de moduladores externos}

Las técnicas de linealización en moduladores MZM tienen como objetivo la mejora del margen dinámico o del SFDR. Para conseguirlo, se actúa sobre diferentes parámetros del modulador MZM, desde la forma de la de RF moduladora ó parámetros constructivos del modulador como la longitud de sus electrodos. Las técnicas de linealización se pueden clasificar en tres grandes grupos según los parámetros modificados: (i) técnicas de linealización eléctrica que actúan sobre la señal RF [Naz93], [Chi99], (ii) técnicas de linealización mediante post-procesado de la señal modulada [Hel00] y (iii) técnicas de linealización todo ópticas que proponen cambios en la estructura interna y en parámetros físicos u ópticos [Kor90], [Oga92], [Bet94], [Shi00].

\subsubsection{Linealización eléctrica y mediante post-procesado}

En esta sección, se comentan el grupo de técnicas de linealización eléctrica que actúan sobre la señal RF y el grupo de técnicas de linealización mediante post-procesado de la señal modulada.

Dentro del grupo de las técnicas de linealización eléctrica que actúan sobre la señal $\mathrm{RF}$, el objetivo es disminuir la IMD del MZM mediante la corrección de la señal RF moduladora. Ejemplos de esta técnica son la predistorsión de la señal de RF mediante un circuito eléctrico de respuesta no-lineal inversa a la que presenta el MZM [Chi99]. Otra técnica es la retroalimentación de un segundo modulador con una señal de error proveniente de comparar la salida modulada del primer modulador con la señal de RF. La realimentación de este error permite al segundo modulador actuar de forma diferente y linealizar la señal [Naz93].

Las técnicas de linealización mediante procesado de la señal modulada son muy diversas y se configuran dependiendo de la aplicación final. Por ejemplo, una de ellas actúa sobre la señal modulada mediante su conversión ADC y procesado digital para mejorar su respuesta lineal [Hel00].

\subsubsection{Linealización óptica}

Las técnicas de linealización óptica para MZM actúan sobre el efecto electro-óptico del modulador. Estas técnicas se basan en la modificación de parámetros constructivos y relacionados con la señal óptica del MZM, como son sus factores de acoplo, el desfase entre ramas y la polarización. A su vez, estas técnicas presentan agrupaciones de moduladores que permiten linealizar la respuesta de la señal modulada. Es importante destacar que esta técnica no modifica la señal RF moduladora. 
Las técnicas ópticas de linealización de MZM se pueden clasificar según el ancho de banda de trabajo en el cual son válidas y también según el tipo de agrupación de MZM que realiza cada técnica.

Según el ancho de banda de trabajo, las técnicas de linealización óptica se clasifican en banda estrecha, si el ancho de banda de trabajo es menor a una octava, o en banda ancha para anchos de banda de trabajo multi-octava. En las técnicas de banda estrecha, el margen dinámico del MZM está limitado por el ruido de la señal moduladora. Por lo tanto, las variaciones de la tensión de polarización reducen el margen dinámico del MZM. Por este motivo, el uso de sistemas de control dinámico del BIAS es recomendable en técnicas de linealización de banda estrecha. En los sistemas MZM de banda estrecha, los productos de intermodulación de segundo orden (IM2, $c_{2}$ ), es decir, aquellas combinaciones de armónicos del tipo $\omega_{1}+\omega_{2}$, quedan fuera de la banda de trabajo. De esta forma, sólo es necesario mitigar el efecto de la distorsión de los productos de intermodulación de tercer orden (IM3, $c_{3}$ ) como $2 \omega_{1}-\omega_{2}$. Este componente armónico de tercer orden se consigue minimizar aplicando la tensión de polarización adecuada para trabajar en la zona MATB o MITB del MZM.

En las técnicas de linealización óptica de banda ancha, se requiere reducir las componentes de intermodulación de segundo y tercer orden $\left(c_{2}, c_{3}\right)$, lo que aumenta la complejidad de la linealización.

En cuanto a la clasificación de las técnicas de linealización ópticas según la disposición de sus moduladores, tenemos agrupaciones de moduladores MZM dispuestos en serie y en paralelo.

El trabajo expuesto en este capítulo de la Tesis Doctoral se centra en técnicas de linealización ópticas de banda ancha, para agrupaciones de moduladores MZM tanto serie como paralelo. La elección de técnicas de linealización de banda ancha viene condicionada por el uso de señales RF de gran ancho de banda, como UWB localizada en la banda de frecuencias de $3.1 \mathrm{GHz}$ a $10.6 \mathrm{GHz}$.

\subsubsection{Configuración en cascada o serie}

Las técnicas de linealización óptica en las cuáles se agrupan los moduladores MZM en cascada o serie, presentan la siguiente función de transferencia:

$$
T_{M Z M_{-} \text {serie_lineal }}=\frac{1}{4}\left[1+\cos \left(\phi_{1}+\frac{\pi}{V_{\pi}} V_{p o l} \sqrt{1-s}\right)\right]\left[1+\cos \left(\phi_{2}+\frac{\pi}{V_{\pi}} V_{p o l} \sqrt{s}\right)\right] \text {, }
$$

resultante de la multiplicación de la función de transferencia individual de cada modulador electro-óptico. 


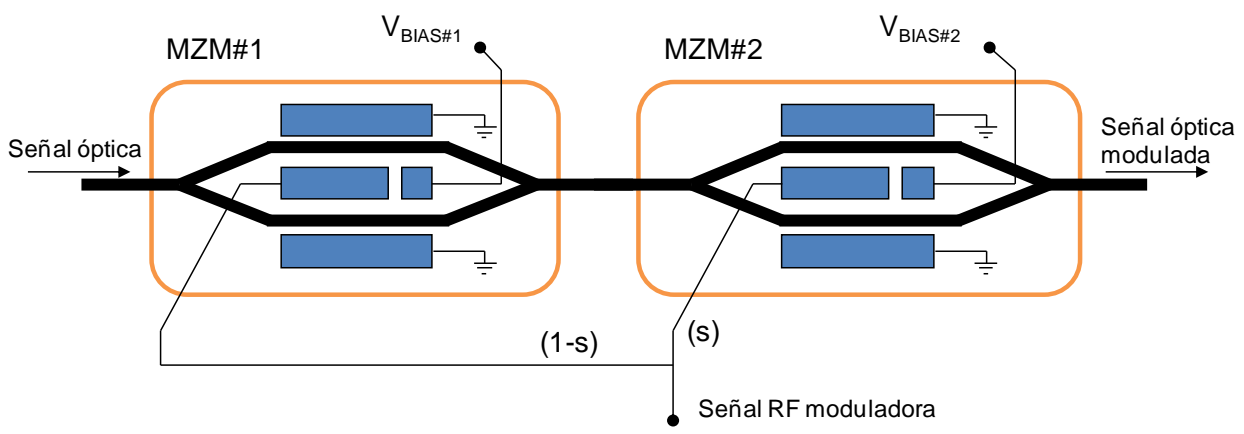

Figura 5.3. Esquema de la propuesta dual serie MZM (DSMZM) para linealización óptica en banda estrecha por [Bet94].

En la Figura 5.3 se presenta el esquema de linealización dual serie MZM (DSMZM, Dual-Serial Mach-Zehnder Modulator) de banda estrecha propuesto en [Bet94]. En este esquema la linealización óptica de banda estrecha se realiza con puntos óptimos de BIAS\#2 para cualquier combinación de BIAS\#1 y un factor de señal RF $s$. De forma práctica los valores de BIAS\#1 y $s$ deben minimizar la figura de ruido del sistema. Una vez conseguido esto, el control de la tensión de polarización BIAS\#2 seleccionada permitirá monitorizar los productos IM3 y reducirlos. Los valores de BIAS \#1 yBIAS\#2 se obtienen de resolver la ecuación (5.18) para coeficientes de $c_{3}=0$, dados por la ecuación (5.13). En [Bet94], se consigue mejorar en $18.5 \mathrm{~dB}$ el margen dinámico (DR, Dynamic Range) para $1 \mathrm{MHz}$, banda estrecha, y sólo se aumenta en $0.4 \mathrm{~dB}$ la figura de ruido del sistema completo.

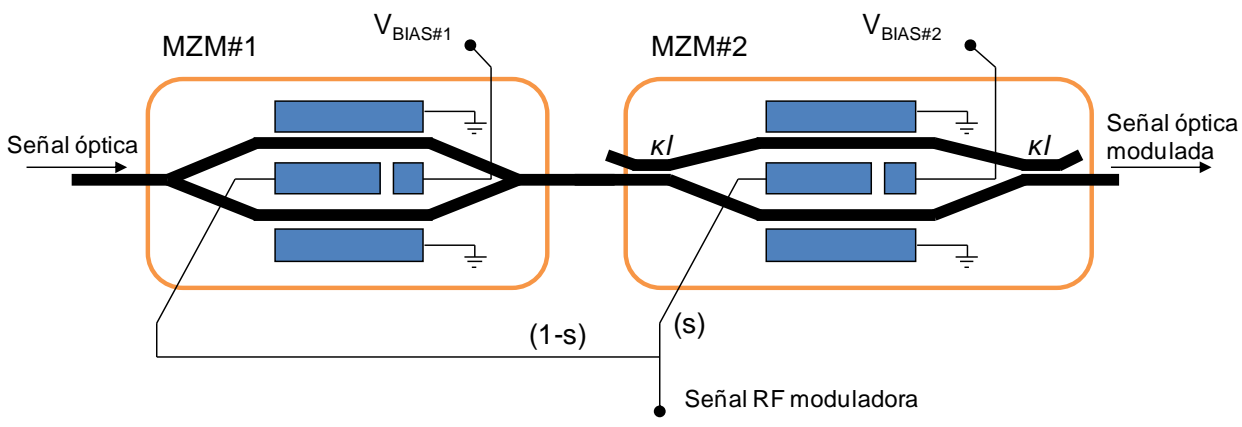

Figura 5.4. Esquema de la propuesta dual serie MZM (DSMZM) para linealización óptica en banda ancha por [Bri92].

La Figura 5.4 muestra un esquema de linealización óptica DSMZM de banda ancha propuesto en [Bri92]. En este esquema se observa cómo el MZM\#2 presenta pérdidas, es decir, que está en una situación de no extinción [Ske91], [Bri92]. En el esquema de la Figura 5.4, se observa la incorporación de acopladores direccionales en las transiciones entre MZM\#1 y MZM\#2, y a la salida del MZM\#2, para reducir las componentes $c_{2}$ y $c_{3}$ 
a cero. Este esquema práctico opta por fijar el punto de extinción ( $k l$, potencia óptica entrante al $2^{\circ}$ modulador) y el factor $s$ del divisor de RF para minimizar la figura de ruido. Una vez fijados, se obtienen los puntos de polarización BIAS\#1 y BIAS\#2 que reducen los componentes de intermodulación $c_{2}$ y $c_{3}$.

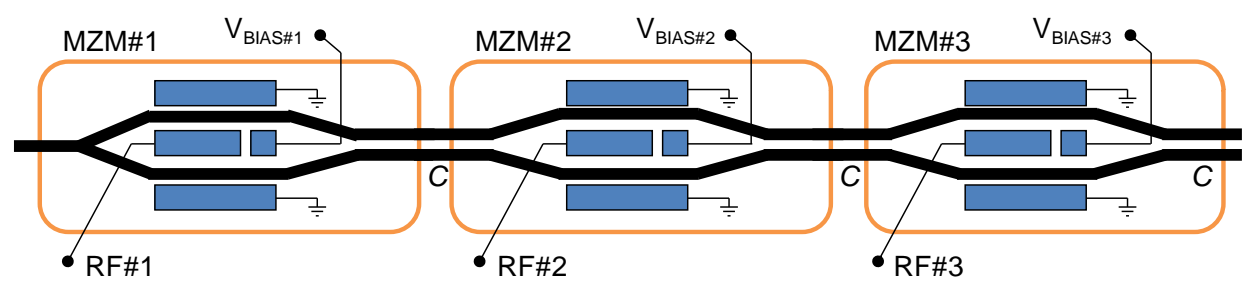

Figura 5.5. Esquema de la propuesta serie MZM mediante acopladores para linealización óptica hasta quinto orden [Bur95].

En la Figura 5.5 se presenta el esquema de linealización serie hasta quinto orden propuesto en [Bur95]. Este esquema se basa en una arquitectura en cascada de 3 MZM, que supone una ampliación de la solución propuesta para linealización con dos MZM en cascada en [Naz94]. En la propuesta original de [Naz94], se utiliza una alimentación de RF de relación 1:-1 en cada modulador, lo cual simplifica el control de la tensión de polarización. En la propuesta de 3 MZM en cascada de [Bur95], la alimentación RF es del tipo 1:-1:1. En el esquema de la Figura 5.5, el ajuste del tercer acoplador, $C_{3}$, optimiza la reducción de la distorsión armónica de hasta quinto orden, proporcionando mejoras de unos $20 \mathrm{~dB}$ en la distorsión de tercer orden (IMD3, Third Order Intermodulation Distortion) para anchos de banda menores de una octava en aplicaciones CATV.

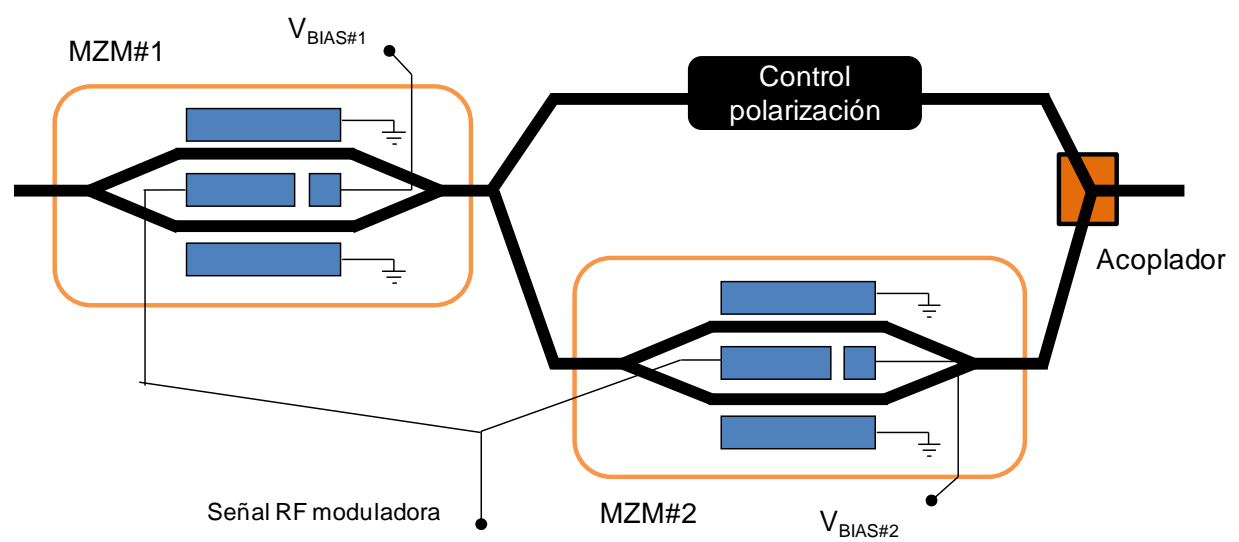

Figura 5.6. Esquema de la propuesta de linealización óptica serie en banda ancha con 2 MZM y una estructura de acopladores [Wan92]. 
En la Figura 5.6 se presenta el esquema de una técnica de linealización serie de banda ancha con 2 MZM y un acoplador [Wan92]. Este esquema propone el uso de un interferómetro como segundo módulo en serie, y sitúa un modulador MZM y un control de la polarización en cada uno de los brazos. El control de la polarización permite asegurar que las señales ópticas sean ortogonales, lo cual proporciona una mayor linealización de acuerdo a [Wan92]. Esta configuración presenta reducciones de la IMD3 de hasta $20 \mathrm{~dB}$, pero aumenta la IMD2, lo que limita su aplicación a sistemas de banda estrecha.

\subsubsection{Configuración en paralelo}

Las técnicas de linealización óptica en paralelo para moduladores MZM presentan la siguiente función de transferencia:

$$
\begin{aligned}
& T_{M Z M_{-} \text {paralelo_lineal }}= \\
& =\frac{\sqrt{a}}{2}\left[1+\cos \left(\phi_{1}+\frac{\pi}{V_{\pi 1}} V_{p o l} \sqrt{1-s}\right)\right]+\frac{\sqrt{(1-a)}}{2}\left[1+\cos \left(\phi_{2}+\frac{\pi}{V_{\pi 2}} V_{p o l} \sqrt{s}\right)\right],
\end{aligned}
$$

resultante de la suma de las funciones de transferencia individual de cada modulador.

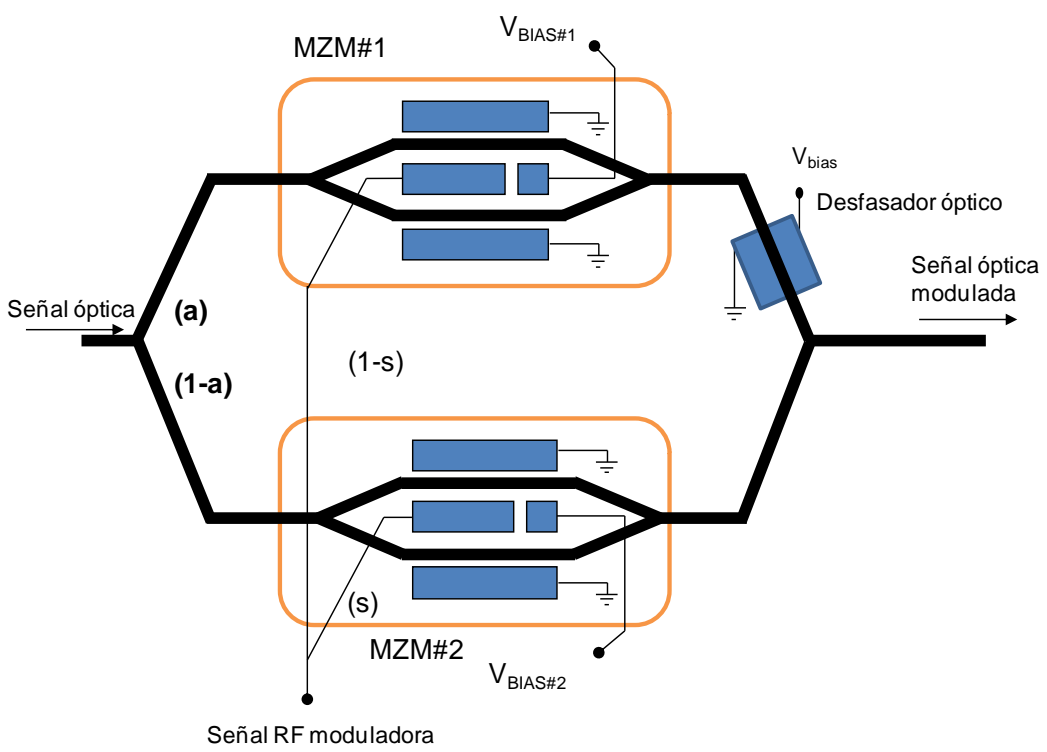

Figura 5.7. Esquema de la propuesta de linealización óptica dual paralelo con 2 MZM (DPMZM) [Kor90].

La principal estructura de linealización óptica dual en paralelo (DPMZM, Dual-Parallel Mach-Zehnder Modulator) fue la propuesta en [Kor90], la cual se 
presenta en la Figura 5.7. En este esquema, cada MZM presenta puntos de polarización diferentes debido a la división de la señal de RF dada por el factor $s$ y al divisor de potencia óptica de factor $a$. La resolución de la ecuación (5.19) con estos parámetros $a$ y $s$, permite obtener la minimización de los componentes de intermodulación $c_{2}$ y $c_{3}$.

El esquema propuesto en [Kor90] se basa en obtener respuestas de carácter contrario en cada rama para que a la salida no predominen los factores de distorsión IM3. Esto se consigue mediante una alimentación asimétrica de señales ópticas y eléctricas en cada MZM. En esta configuración, cuando la rama superior recibe mayor parte de potencia óptica, la rama inferior recibe la mayor parte de la señal RF. De esta forma, se minimiza el IMD3, pero se reduce al mismo tiempo la ganancia total del sistema. En este esquema, el control de la tensión de polarización BIAS\#1 y BIAS\#2 es independiente para cada MZM. A su vez, se controla la fase de la señal óptica modulada en la rama superior para minimizar el factor $c_{3}$. La complejidad de este esquema reside en el control de los divisores de señal RF y óptica, el control del BIAS y el reajuste de fase óptica como parte de un bucle de control de errores en el sistema.

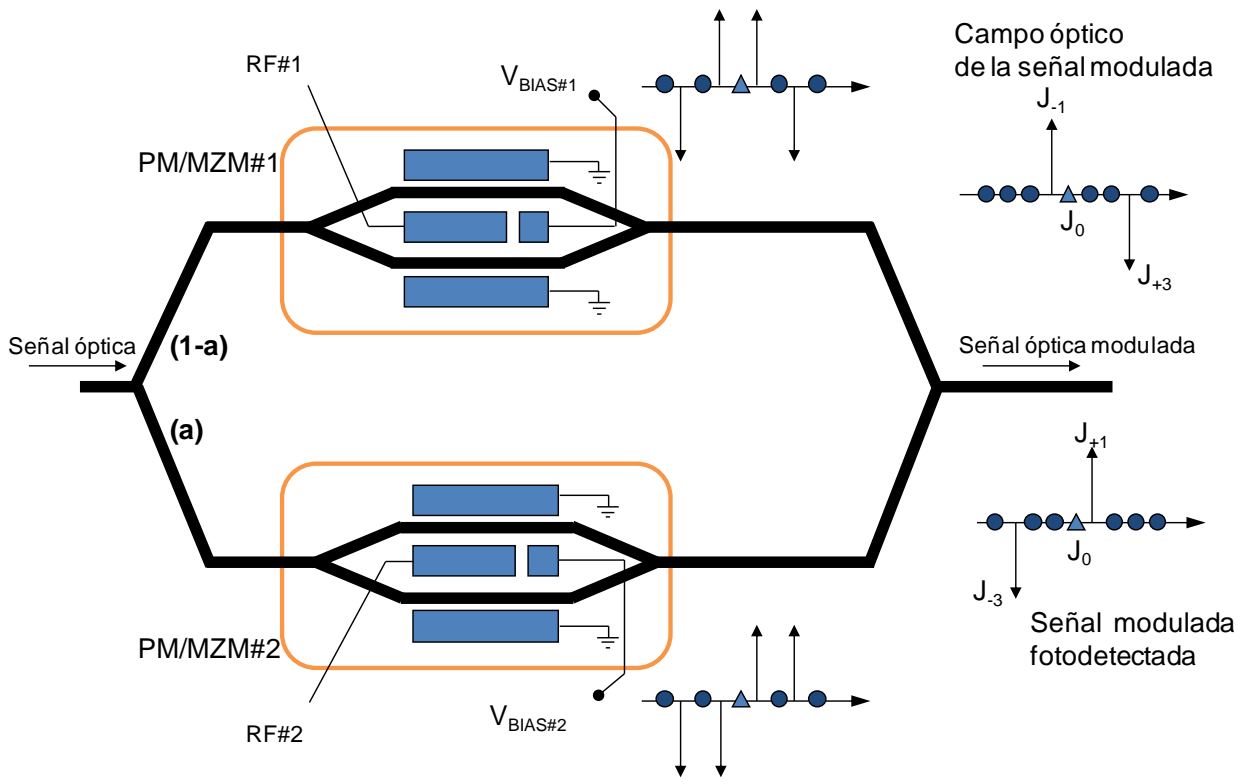

Figura 5.8. Esquema de la propuesta de linealización óptica dual paralelo con 2 MZM (DPMZM) y modulación SSB. Diagrama de funciones Bessel de cada rama del MZM y de la señal óptica final tanto en campo como en RF [Shi01].

En la Figura 5.8 se presenta una técnica de linealización óptica DPMZM que se basa en el uso de modulación de una sola banda lateral (SSB, Single-Side Band). Este tipo de modulación utiliza una única banda lateral, lo que reduce los efectos no-lineales 
y requiere menos potencia óptica que una modulación convencional. Los trabajos de [Oga92], [Shi00] y [Shi01] presentan ejemplos de técnicas de linealización óptica mediante modulación SSB. En la Figura 5.8 se presenta el esquema propuesto en [Shi01]. En este caso, se genera una modulación SSB con supresión de portadora (SSB-SC, SSB Supression Carrier) mediante el uso de 2 moduladores de fase MZM (PM/MZM, Phase Modulator MZM) en paralelo. En los diagramas de las funciones de Bessel de la Figura 5.8 se observa cómo la configuración adecuada de la respuesta de cada MZM permite conseguir una modulación SSB mediante la cual se reduce la componente IM3, $c_{3}$. En concreto, se consigue una reducción de IMD3 de -18.4 dB. Sin embargo, la penalización en potencia óptica es $-3 \mathrm{~dB}$ sobre la potencia máxima de los moduladores convencionales utilizados.

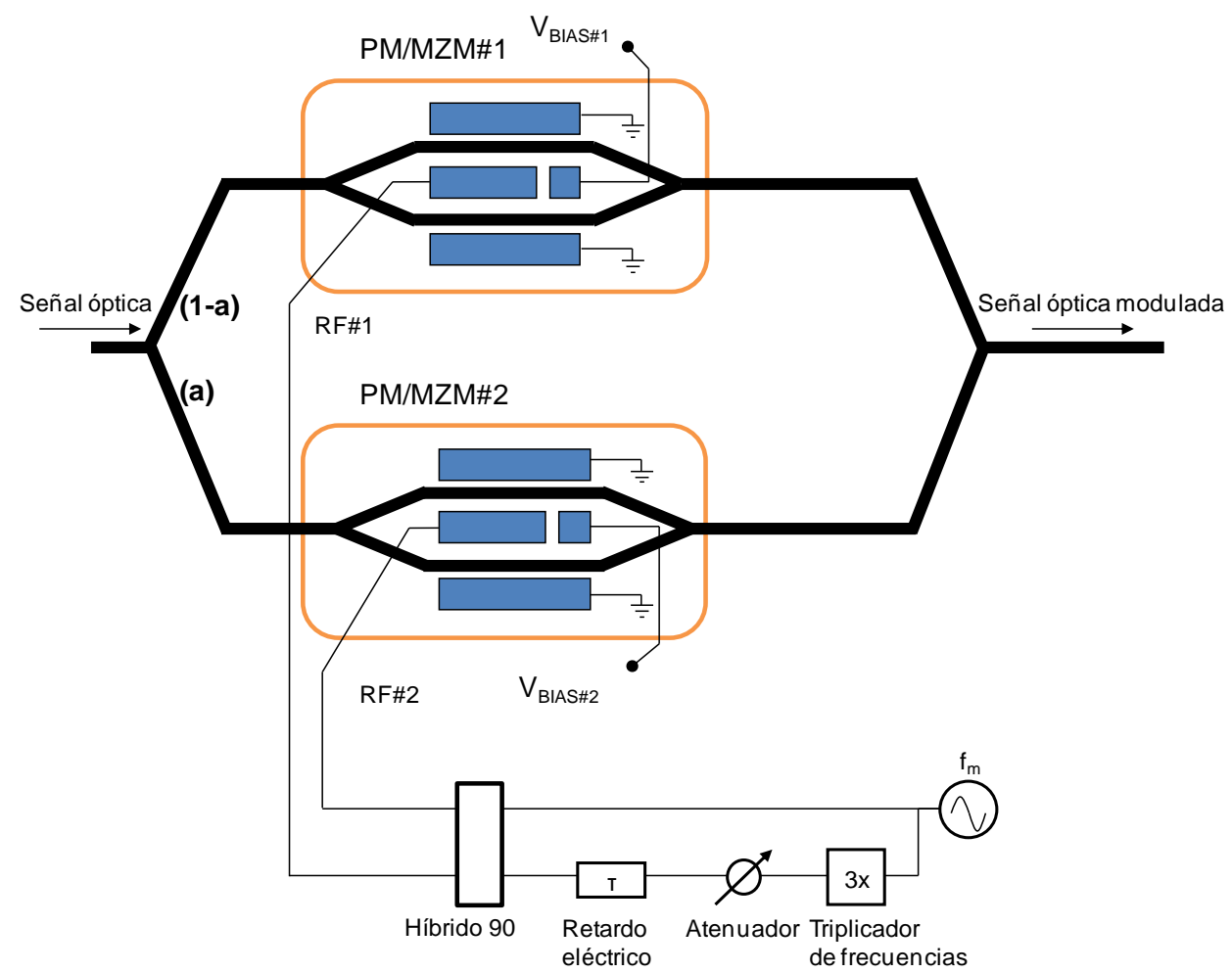

Figura 5.9. Esquema de la propuesta de linealización óptica dual paralelo con 2 MZM (DPMZM) y modulación SSB [Kaw03].

En la Figura 5.9 se propone una arquitectura DPMZM con dos moduladores PM/MZM y modulación SSB para reducir el ruido del sistema y la distorsión de IMD3 [Kaw03]. Su funcionamiento se basa en aplicar dos señales moduladoras RF, siendo su frecuencia $\omega_{1}$ y $\omega_{2}=3 * \omega_{1}$, pero desfasadas $90^{\circ}$. Así, además de las componentes Bessel 
de $\omega_{1}$ de la rama inferior, $\mathbf{J}_{1}(\mathrm{~A})$ y $\mathbf{J}_{3}(\mathrm{~A})$, la segunda señal de $\mathrm{RF} \omega_{2}=3 * \omega_{1}$ permite compensar la componente $f_{0^{-}} 3 f_{m}$. La amplitud del armónico fundamental cuando usamos la señal de RF con frecuencia $\omega_{2}$ es $J_{1}(B)$, el cual se sitúa en la misma frecuencia que $\mathrm{J}_{3}(\mathrm{~A})$. Si $\mathrm{J}_{3}(\mathrm{~A})$ y $\mathrm{J}_{1}(\mathrm{~B})$ son iguales y la fase entre los osciladores que los generan es $180^{\circ}$, el componente $f_{0}-3 f_{m}$ se anula, linealizando la respuesta del sistema. En [Kaw03] se presentan resultados de reducción del tercer armónico $f_{0}+3 f_{m}$ de $10.4 \mathrm{~dB}$ para un oscilador local de $\omega_{1}=6 \mathrm{GHz}$.

Otra técnica de linealización óptica de banda ancha es la estructura propuesta en [Ack99], que se basa en un modulador MZM Travelling-Wave (TW-MZM) con dos polarizaciones. Esta técnica se complementa con una detección en paralelo para cada polarización y la utilización de un acoplador para mejorar el SNR. En [Ack99] se presenta un SFDR de $74 \mathrm{~dB}$ en un ancho de banda de $800-2500 \mathrm{MHz}$ para una figura de ruido de $35 \mathrm{~dB}$.

Existen otras técnicas de linealización óptica que abarcan parámetros constructivos del modulador. Un ejemplo es el uso de interferómetros MZM con anillos resonantes en sus brazos, que proporcionan mejoras en linealización sin necesidad de un control eléctrico complejo [Xie03]. En esta configuración, el campo eléctrico de salida tiene un desfase inducido y controlado por el anillo resonante de cada brazo, $\Phi_{a}$ y $\Phi_{b}$, que depende de la tensión aplicada. Para trabajar en QB, este modulador necesita que los anillos estén en no-resonancia (off-resonance state), y para ello deben configurar un camino óptico de longitud múltiplo impar de la mitad de la longitud de onda de operación. Analizando la corriente a la salida del modulador y su relación con el cambio del índice de refracción por la tensión aplicada a los anillos resonantes, $\Delta n(V)$, se obtiene la condición para minimizar la IMD3. Esta condición proporciona los parámetros necesarios para el diseño de los anillos resonantes, como son su radio y coeficiente de acoplo. Los resultados obtenidos indican mejoras mayores de $15 \mathrm{~dB}$ frente a un MZM convencional con la arquitectura de dos anillos, y también indican que esta configuración es más robusta frente a desviaciones en el coeficiente de acoplo de cada anillo. Sin embargo, su respuesta en frecuencia presenta un ancho de banda la mitad que un MZM convencional. En la propuesta de [Xie03] se trabaja en frecuencias de $50 \mathrm{GHz}$ con una $V_{\pi}$ de $5 \mathrm{~V}$. 


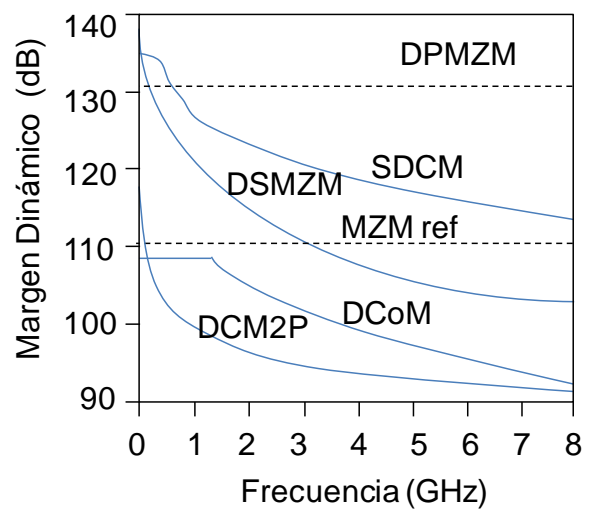

Figura 5.10. Resumen de técnicas de linealización ópticas basadas en moduladores y acopladores direccionales serie y paralelo, expuestas en [Cum98].

En la Figura 5.10 se presenta el resumen de técnicas de linealización óptica propuesta en [Cum98], donde se evalúan arquitecturas DPMZM y DSMZM junto con otras de acopladores. En esta figura se observa cómo para aplicaciones de banda ancha las configuraciones paralelo presentan un mayor SFDR y son más estables que las configuraciones serie. Las técnicas de linealización basadas en acopladores direccionales que se muestran en la Figura 5.10, son un único modulador de acoplador direccional con 2 puntos de polarización (DCoM, Simple Directional Coupler Modulator), acopladores direccionales para banda estrecha (SDCM, Sub-octave Directional Coupler Modulator) y dual DCoM serie (DCM2P, Directional Coupler Modulator with Two Passive Bias Sections in Cascade).

La Tabla 5.1 y Tabla 5.2 resumen las principales características de cada esquema de linealización óptica expuesto en esta sección.

TABLA 5.1. COMPARATIVA PRESTACIONES ARQUITECTURAS PARALELO DE LINEALIZACIÓN ÓPTICA EN MODULADORES ELECTRO-ÓPTICOS

\begin{tabular}{c|c|c|c|c}
\hline \hline Propuesta & {$[$ Bro93] } & {$[$ Ack99] } & {$[$ Jun02] } & [Xie03] \\
\hline $\begin{array}{c}\text { Arquitectura } \\
\text { paralelo }\end{array}$ & $2 \mathrm{EAM}$ & $\begin{array}{c}1 \mathrm{TW}-\mathrm{MZM} \\
\text { ó } \\
2 \mathrm{MZM}\end{array}$ & $2 \mathrm{EAM}$ & $\begin{array}{c}\text { MZI con } 2 \text { brazos } \\
\text { resonadores en anillo }\end{array}$ \\
\hline Ancho de banda & $\begin{array}{c}\text { CATV } \\
450 \mathrm{MHz}\end{array}$ & Multi-octava & Sub-octava & $\mathrm{f}_{0} \approx 50 \mathrm{GHz}$ \\
\hline Supresión IMD & $20 \mathrm{~dB} \mathrm{CTB}$ & $8 / 9 \mathrm{~dB}$ IMD & $14.5 \mathrm{~dB}$ IMD3 & Mejora 5 10 dB IMD3 \\
\hline \hline
\end{tabular}


TABLA 5.2. COMPARATIVA PRESTACIONES ARQUITECTURAS SERIE DE LINEALIZACIÓN ÓPTICA EN MODULADORES ELECTRO-ÓPTICOS

\begin{tabular}{c|c|c|c|c}
\hline \hline Propuesta & {$[$ Wan92] } & [Bur95] & [Ack99] & [Soh00] \\
\hline Arquitectura & $2 \mathrm{MZM}+\mathrm{PC}$ & $3 \mathrm{MZM}$ en cascada & TW-MZM & 2 EAM en cascada \\
\hline Ancho de banda & $\begin{array}{c}\mathrm{CATV} \\
(<1 \mathrm{GHz})\end{array}$ & $\begin{array}{c}\text { CATV } \\
(>1 \mathrm{GHz})\end{array}$ & Multi-octava & Sub-octava \\
\hline Supresión de IMD3 & $20 \mathrm{~dB}$ & $13 \mathrm{~dB}$ & $20 \mathrm{~dB}$ & $10 \mathrm{~dB}$ \\
\hline \hline
\end{tabular}

Para complementar este estudio de técnicas de linealización óptica, es recomendable la revisión realizada en [Bet02] y [Cox04] sobre moduladores externos fabricados en $\mathrm{LiNbO}_{3}$.

\subsection{Análisis de técnica de linealización óptica dual en paralelo MZM}

La evaluación propuesta en esta sección se basa en técnicas de linealización ópticas de banda ancha, puesto que en esta Tesis Doctoral se ha evaluado la distribución y coexistencia de señales UWB y WiMAX, centradas en las bandas de frecuencias de $3.1 \mathrm{GHz}-10.6 \mathrm{GHz}$ y $3.5 \mathrm{GHz}$, respectivamente, en redes de acceso integradas. Por otro lado, este estudio se centra en una técnica de arquitectura paralelo porque teóricamente permite una mayor reducción de la IMD3 en situaciones multi-octava.

La técnica de linealización óptica dual paralelo DPMZM propuesta y evaluada en esta sección, se basa en el esquema de [Kor90]. El objetivo es estudiar las limitaciones y mejoras que se pueden obtener utilizando componentes comerciales y no desarrollos específicos para realizar la linealización de un MZM. Como se comentó en la sección anterior, el esquema de [Kor90] permite mejoras de hasta $10 \mathrm{~dB}$ en el DR con respecto a esquemas con un solo MZM [Cox04]. La técnica DPMZM propuesta incluye la foto-detección de cada una de las ramas de forma separada, para que al combinarlas de forma diferencial se reduzca el efecto de IMD3. De esta forma, se consigue una ampliación del esquema propuesto en [Kor90]. 


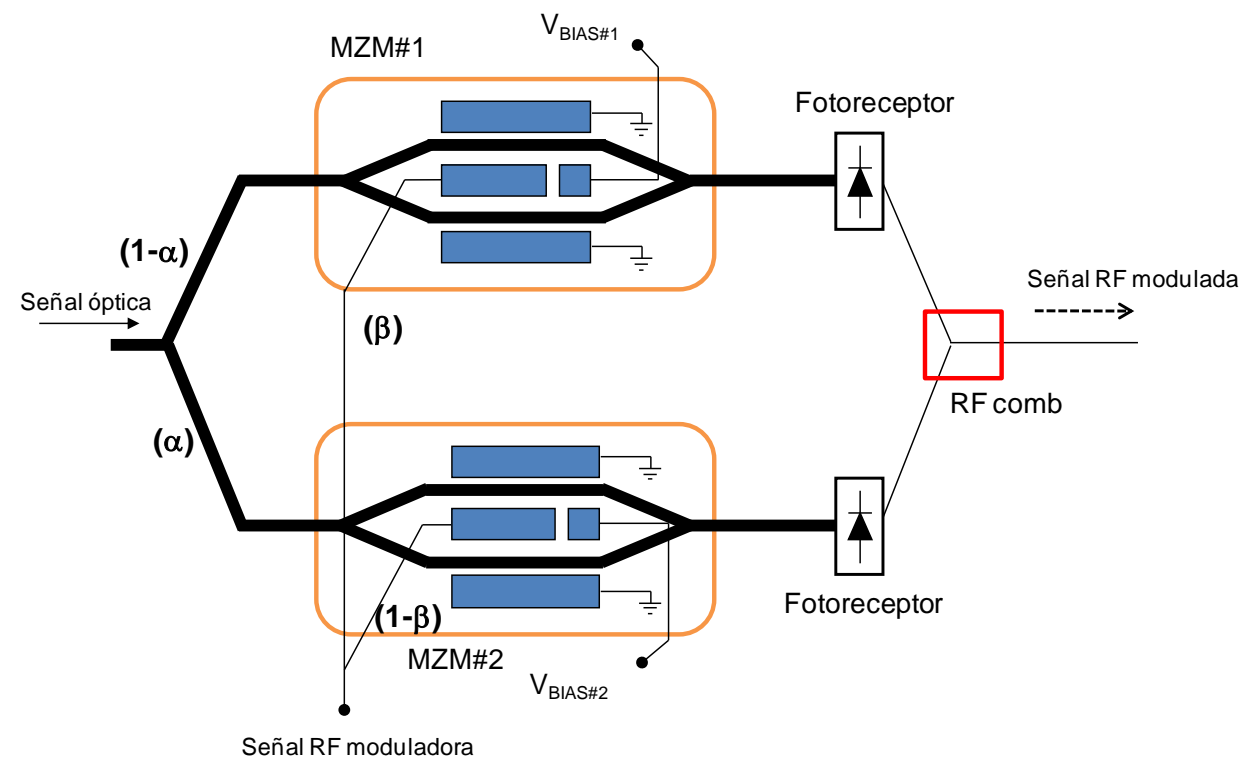

Figura 5.11. Concepto de linealización óptica DPMZM con alimentación óptica y RF asimétrica y foto-detección diferencial.

En la Figura 5.11 se presenta el montaje de linealización óptica DPMZM en banda ancha propuesto y evaluado. Cabe destacar que se propone una alimentación asimétrica de ambos moduladores, tanto en señal RF como en señal óptica, de acuerdo con los factores $\alpha$ y $\beta$. Ambos MZM se alimentan asimétricamente de forma inversa, es decir, cuando un MZM recibe mayor potencia óptica, a su vez, está recibiendo la menor potencia RF posible. El objetivo de la alimentación asimétrica eléctrica es obtener una señal modulada que no excite los términos de quinto orden en un MZM. En cambio, el objetivo de la alimentación asimétrica óptica es disminuir la potencia de las componentes IM3 en el otro MZM. Esto permite que tras foto-detectar y sumar ambas señales moduladas, se consiga aumentar la relación IMD3, debido a que las contribuciones por distorsión armónica son menores. Por último, la foto-detección diferencial permite reducir el ruido RIN del sistema en el que se aplica [Jos05], por ejemplo, para un láser de $-135 \mathrm{~dB} / \mathrm{Hz}$ de ruido intrínseco relativo (RIN, Relative Intensive Noise) con $P_{\text {out }}$ de $5 \mathrm{dBm}$, se obtienen mejoras de $20 \mathrm{~dB}$ en SNR. Esta mejora de SNR permite conseguir mayores valores de SFDR que con un solo fotodiodo, lo cual, unido a la mejora introducida con el esquema linealizado, aumenta significativamente el margen dinámico del sistema. 


\subsubsection{Evaluación analítica y mediante simulación}

En un primer paso, el esquema de linealización propuesto se analiza desde el punto de vista de la función de transferencia, evaluando las contribuciones de los productos de intermodulación en base a la alimentación asimétrica.

A partir de la ecuación (5.8), se realiza el desarrollo de la potencia de salida óptica en funciones de Bessel para $V_{p o l}=V_{1}-V_{2}$, es decir, asumiendo un comportamiento dual-drive del modulador [Cor01]. De esta forma, se obtiene la siguiente expresión, donde $m_{i}=V_{i} / V_{\pi}$ y la señal RF de entrada al modulador se basa en dos portadoras de RF $\omega_{1}$ y $\omega_{2}$ :

$$
\frac{\pi}{V_{\pi}}\left(V_{1}-V_{2}\right)=m_{D C}+m_{1} \sin \left(\omega_{1} t+\phi_{1}\right)+m_{2} \sin \left(\omega_{2} t+\phi_{2}\right)
$$

Teniendo en cuenta la expresión de la potencia de salida óptica dada por la ecuación (5.8), y reescribiendo la ecuación (5.20) en base a funciones de Bessel, se obtiene la siguiente expresión de la potencia de salida del modulador dual-drive:

$P_{\text {out }}=P_{\text {in }} \cdot L_{f f}$.

$$
\left(\begin{array}{c}
1+\cos \left(m_{D C}\right)\left(\begin{array}{l}
\sum_{j=-\infty}^{\infty} \sum_{k=-\infty}^{\infty} J_{2 j}\left(m_{1}\right) J_{2 k}\left(m_{2}\right) \cos \left(2 j \omega_{1} t+2 k \omega_{2} t\right)+ \\
+\sum_{j=-\infty}^{\infty} \sum_{k=-\infty}^{\infty} J_{2 j+1}\left(m_{1}\right) J_{2 k+1}\left(m_{2}\right) \cos \left((2 j+1) \omega_{1} t+(2 k+1) \omega_{2} t\right)
\end{array}\right)+ \\
+\sin \left(m_{D C}\right)\left(\begin{array}{l}
\sum_{j=-\infty}^{\infty} \sum_{k=-\infty}^{\infty} J_{2 j+1}\left(m_{1}\right) J_{2 k}\left(m_{2}\right) \sin \left((2 j+1) \omega_{1} t+2 k \omega_{2} t\right)+ \\
+\sum_{j=-\infty}^{\infty} \sum_{k=-\infty}^{\infty} J_{2 j}\left(m_{1}\right) J_{2 k+1}\left(m_{2}\right) \cos \left(2 j \omega_{1} t+(2 k+1) \omega_{2} t\right)
\end{array}\right)
\end{array}\right)
$$

En esta ecuación (5.21) se observan claramente dos términos, uno relacionado con los armónicos de tipo par, los que acompañan a la expresión coseno, y otro relacionado con los armónicos de tipo impar, los que acompañan a la expresión seno. Los índices $j, k$ hacen referencia al orden del armónico.

Ante un modulador con tensión de polarización QB, es decir, con $m_{D C}=\pi / 2$, se observa cómo desaparecen los términos armónicos de tipo impar de la ecuación (5.21). Si se configura el modulador dual-drive en QB con dos portadoras RF como se indica en la ecuación (5.20), se puede foto-detectar y analizar la potencia de salida óptica dada por la ecuación (5.21) a partir del desarrollo mostrado en las ecuaciones (5.14)-(5.16). Este análisis permite obtener los productos de distorsión para la intermodulación de segundo orden $\left(\omega_{1} \pm \omega_{2}\right.$, IM2 $)$ y tercer orden $\left(2 \cdot \omega_{1,2} \pm \omega_{2,1}\right.$, IM3 $)$ y para los armónicos de 
segundo orden $\left(2 \cdot \omega_{2,1}, \mathrm{H} 2\right)$ y tercer orden $\left(3 \cdot \omega_{1,2}, \mathrm{H} 3\right)$, que se muestran en las ecuaciones siguientes:

$$
\begin{aligned}
& H_{0}=-\sin \left(m_{D C}\right) J_{1}(m) J_{0}(m), \\
& H 2=-\cos \left(m_{D C}\right) J_{2}(m) J_{0}(m), \\
& H 3=-\sin \left(m_{D C}\right) J_{3}(m) J_{0}(m), \\
& I M 2= \pm \cos \left(m_{D C}\right) J_{1}^{2}(m), \\
& I M 3=\mp \sin \left(m_{D C}\right) J_{2}(m) J_{1}(m) .
\end{aligned}
$$

Tras estudiar el comportamiento a nivel de señal del MZM dual-drive, se analiza a partir de la ecuación (5.21) el esquema de linealización óptica DPMZM propuesto introduciendo la alimentación asimétrica en base a los factores $\alpha$ y $\beta$, cuyo valor varía entre 0 y 1 . En este análisis ambos MZM trabajan en QB, y la señal óptica modulada es detectada por un fotodiodo de responsividad $R$. Analizando la señal de corriente foto-detectada, se extraen los términos correspondientes a los armónicos de primer y tercer orden, que se corresponden con las ecuaciones (5.23) y (5.24), respectivamente:

$$
\begin{aligned}
& \mathrm{i}_{\text {out }}\left(\omega_{1}\right)=P_{\text {opt }} \Re L_{f f}\left[-\alpha J_{1}(\beta m) J_{0}(\beta m)+(1-\alpha) J_{1}((1-\beta) m) J_{0}((1-\beta) m)\right], \\
& \mathrm{i}_{\text {out }}\left(2 \omega_{1}-\omega_{2}\right)=P_{\text {opt }} \Re L_{f f}\left[\alpha J_{2}(\beta m) J_{1}(\beta m)+(1-\alpha) J_{2}((1-\beta) m) J_{1}((1-\beta) m)\right] .
\end{aligned}
$$

De estas ecuaciones se pueden extraer las relaciones de distorsión de tercer orden (IMD3, HD3), como se observa en las expresiones (5.25) y (5.26):

$$
\begin{aligned}
& I M D 3=i_{\text {out }}\left(\omega_{1}\right) / i_{\text {out }}\left(2 \omega_{1}-\omega_{2}\right), \\
& \text { HD3 }=i_{\text {out }}\left(\omega_{1}\right) / i_{\text {out }}\left(3 \omega_{1}\right) .
\end{aligned}
$$

A continuación, se representa gráficamente el comportamiento de IMD3 y $\mathrm{H}_{0}$ en función de $\alpha$ y $\beta$, lo cual permite obtener resultados del esquema propuesto en la Figura 5.11 cuando ambos MZM trabajan en QB. 


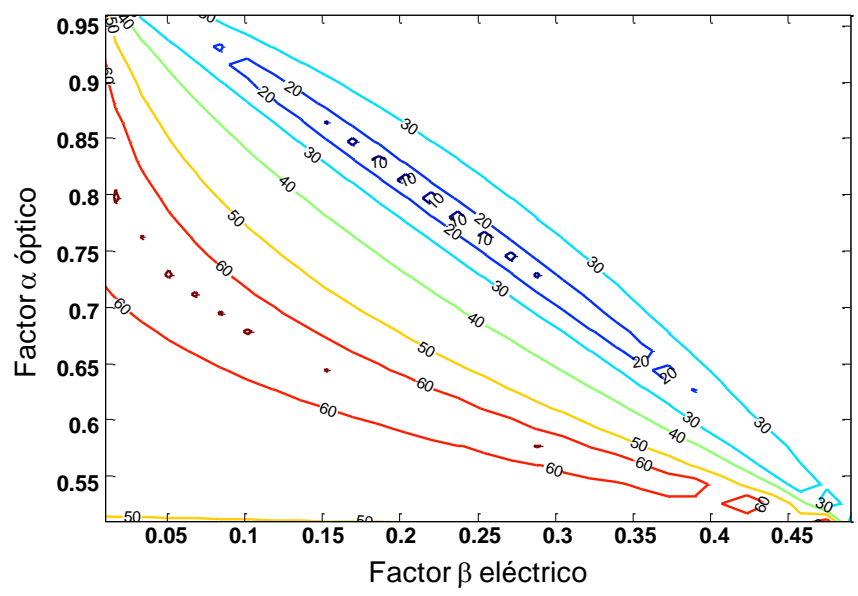

Figura 5.12. Valores analíticos de IMD3 para el esquema de linealización óptica DPMZM propuesto, con $f_{1}=1 \mathrm{GHz}$ y $\mathrm{f}_{2}=1.1 \mathrm{GHz}$.

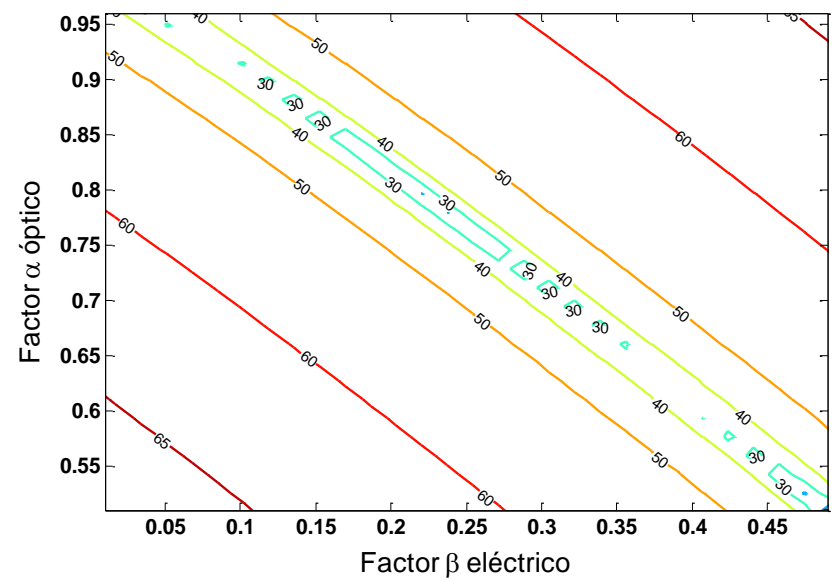

Figura 5.13. Valores analíticos de $H_{0}$ para el esquema de linealización óptica DPMZM propuesto, con $f_{1}=1 \mathrm{GHz}$ y $f_{2}=1.1 \mathrm{GHz}$.

El análisis presentado en la Figura 5.12 y en la Figura 5.13 se ha realizado sobre el esquema de linealización propuesto para un índice de modulación dado, $m_{D C}=\pi / 2$, y para el caso particular de un MZM sin pérdidas. En la Figura 5.12 se observa cómo con factores de alimentación asimétrica, de 0.65 de señal óptica y 0.15 de señal RF, se obtiene una mejora de unos $30 \mathrm{~dB}$ en IMD3 sobre un modulador MZM convencional. En la Figura 5.13 se observa que con estos factores de alimentación asimétrica, se 
aseguran valores de $60 \mathrm{~dB}$ de portadora. En este punto, cabe recordar que estos resultados se corresponden con un caso ideal para banda ancha.

En un siguiente paso, se ha realizado el análisis del esquema de linealización óptica DPMZM con alimentación asimétrica mediante herramientas de simulación óptica. Para ello, se ha escogido una herramienta de simulación óptica basada en Split-Step Fourier [VPI], que permite modelar todos los componentes ópticos y eléctricos del sistema.

El sistema se ha modelado en la herramienta de simulación de acuerdo con los dispositivos disponibles en laboratorio, para facilitar su posterior corroboración experimental. Por ello, se ha simulado un modelo de MZM con $V_{\pi}=1.5 \mathrm{~V}_{\mathrm{DC}}$, una relación de extinción de $20 \mathrm{~dB}$ y unas pérdidas de inserción de $6 \mathrm{~dB}$, lo cual permite compararlo con modelos comerciales como el MZM de COVEGA LN058. A su vez, se ha simulado un generador de señales de RF centrado en $5 \mathrm{GHz}$ con $V_{p p}=1 \mathrm{~V}$ para analizar el HD3 de la arquitectura propuesta. La fuente óptica láser se modela mediante un láser de CW en la longitud de onda de $1550 \mathrm{~nm}$ con una potencia óptica de $10 \mathrm{~mW}$ ( $13 \mathrm{dBm})$. Finalmente, el uso de atenuadores eléctricos y ópticos permite evaluar la alimentación asimétrica mediante los factores $\alpha$ y $\beta$, de acuerdo con el rango de valores analíticos propuesto anteriormente. Los resultados obtenidos en esta simulación óptica se muestran en la Tabla 5.3.

\section{TABLA 5.3. VALORES HD3 OBTENIDOS EN SIMULACIÓN PARA FACTORES DE ALIMENTACIÓN ASIMÉTRICA ÓPTIMOS EN EL ESQUEMA DE LINEALIZACIÓN PARALELO PROPUESTO}

\begin{tabular}{c|c|c}
\hline \hline Atenuación RF $(\boldsymbol{\beta}, \mathbf{d B})$ & Atenuación Óptica $(\boldsymbol{\alpha}, \mathbf{d B})$ & $\mathbf{H D 3}(\mathbf{d B})$ \\
\hline 3 & 18.5 & 37.63 \\
\hline 4 & 20.5 & 39.60 \\
\hline 5 & 21 & 41.66 \\
\hline 6 & 25 & 43.67 \\
\hline 7 & 26 & 45.60 \\
\hline \hline
\end{tabular}

Los resultados mostrados en la Tabla 5.3 indican el valor óptimo de atenuación óptica para un valor de atenuación eléctrica dado. Se observa cómo estos resultados mejoran el valor de HD3 conforme aumenta la atenuación eléctrica y óptica, al igual que se había demostrado de forma analítica en la Figura 5.12. Por ejemplo, para una atenuación eléctrica de $3 \mathrm{~dB}$, equivalente a $\beta=0.25$, con un valor de $18.5 \mathrm{~dB}$ de atenuación óptica, equivalente a $\alpha=0.49$, se obtiene un valor de HD3 de $37.63 \mathrm{~dB}$, que es ligeramente inferior al obtenido de forma analítica. Las diferencias observadas entre los resultados analíticos y los simulados son consecuencia del modelo de simulación que contempla las pérdidas, desviaciones en la tensión de polarización y otras fuentes de 
ruido inherentes a la transmisión óptica, más acorde con los dispositivos disponibles comercialmente en la actualidad.

\subsubsection{Evaluación experimental}

En la Figura 5.14 se presenta el montaje de laboratorio que se ha realizado para evaluar experimentalmente la arquitectura de linealización óptica DPMZM propuesta. En este montaje se incluyen dos MZM de baja $V_{\pi}$, de 1.5 a $2 \mathrm{~V}_{\mathrm{DC}}$, con pérdidas de inserción de 6 a $8 \mathrm{~dB}$ y trabajando ambos moduladores en el punto QB. Se ha utilizado un láser $\mathrm{CW}$ que proporciona $6 \mathrm{dBm}$ de potencia óptica a $\lambda=1550 \mathrm{~nm}$. La señal $\mathrm{RF}$ moduladora se implementa mediante un generador de señales vectoriales Agilent ESG 4438C. En este caso, se ha generado una señal RF de $5 \mathrm{GHz}$ de frecuencia central con una potencia eléctrica máxima de $15 \mathrm{dBm}$. Los factores de alimentación asimétrica se han implementado mediante atenuadores variables eléctricos y ópticos. Las señales ópticas se han foto-detectado mediante dos fotodiodos de responsividad $0.7 \mathrm{~A} / \mathrm{W}$ con un ancho de banda de $45 \mathrm{GHz}$. Por último, las señales RF moduladas son captadas y medidas en un analizador de señales RF, RFSA. Cabe destacar la presencia de retardos eléctricos y ópticos con la intención de ajustar manualmente las derivas temporales introducidas por la fibra óptica y los cables de RF utilizados.

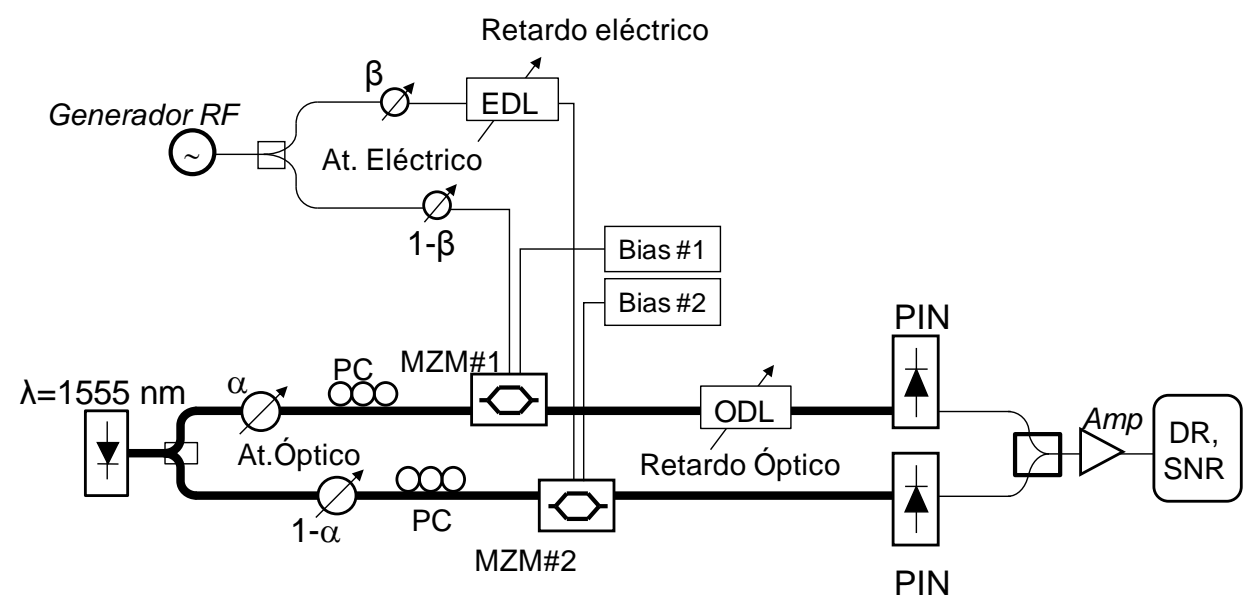

Figura 5.14. Esquema del montaje experimental realizado para evaluar la técnica de linealización óptica DPMZM con alimentación asimétrica.

En un primer paso, este montaje requiere fijar la atenuación eléctrica deseada y variar la atenuación óptica para minimizar la variación del punto de polarización QB de cada modulador. Este punto de polarización se reajusta manualmente para cada paso de atenuación eléctrica medido. La caracterización inicial de los MZM utilizados, proporciona un valor de HD3 de $31 \mathrm{~dB}$ para una señal de RF de $5 \mathrm{GHz}$. Se ha escogido un intervalo de atenuaciones ópticas con valores de $0.5 \mathrm{~dB}$ a $22 \mathrm{~dB}$ y un intervalo de 
atenuaciones eléctricas con valores de $2 \mathrm{~dB}$ a $6 \mathrm{~dB}$. Estos valores se han escogido de acuerdo a los factores de alimentación asimétrica de señal óptica y eléctrica analizados en la sección anterior.

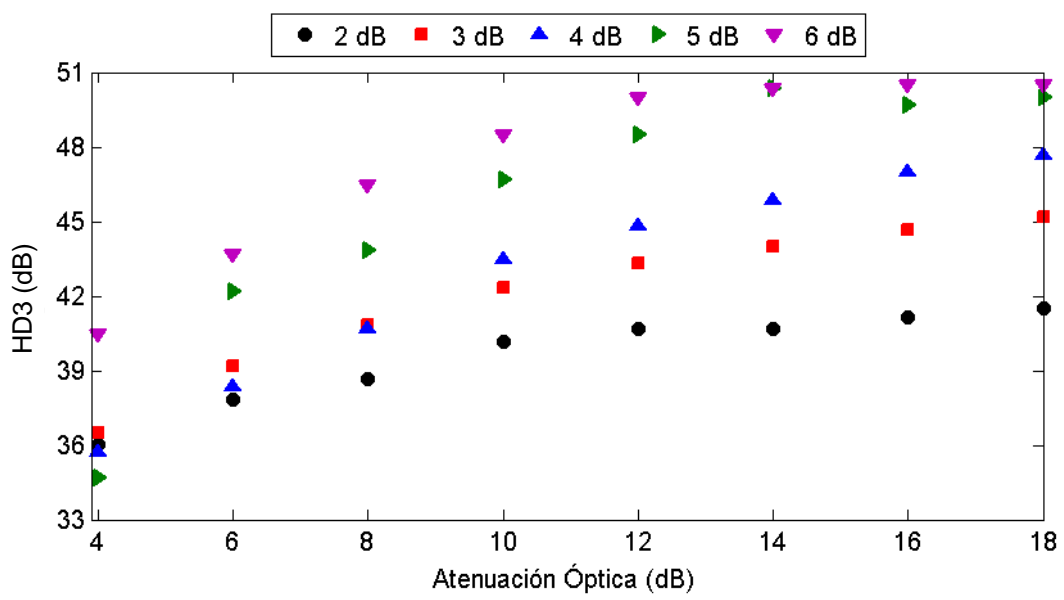

Figura 5.15. Resultados HD3 medidos para diferentes atenuaciones ópticas según una atenuación RF fija.

La Figura 5.15 muestra los resultados HD3 obtenidos para valores de atenuación eléctrica fija en el intervalo $\{2,3,4,5,6\} \mathrm{dB}$, sobre un rango de atenuaciones ópticas entre $4 \mathrm{~dB}$ y $18 \mathrm{~dB}$. El comportamiento de HD3 ante este rango de factores de atenuación sigue la misma tendencia que los valores mostrados analíticamente, pero con una penalización cercana a $6 \mathrm{~dB}$. Estos valores indican una mejora de $20 \mathrm{~dB}$ sobre el valor HD3 para un solo modulador.

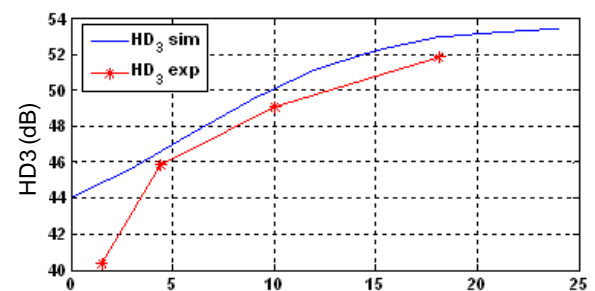

(a)

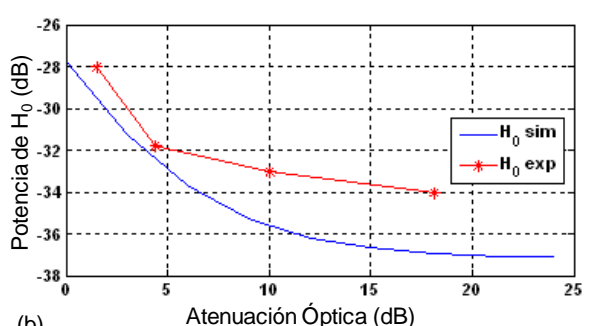

(b)
Atenuación Óptica (dB)

Figura 5.16. Comparativa de resultados (a) HD3 y (b) potencia de portadora $\mathrm{H}_{0}$ en simulación óptica y de forma experimental para una atenuación de RF fija de $6 \mathrm{~dB}, \operatorname{con} f_{R F}=5 \mathrm{GHz}$.

En la Figura 5.16 se comparan los resultados experimentales y de simulación óptica obtenidos para una atenuación eléctrica fija de $6 \mathrm{~dB}$, equivalente a $\beta=0.15$, al variar la atenuación óptica. Se observa como ambos resultados siguen la tendencia de mejorar la relación HD3 a medida que aumenta la atenuación eléctrica y óptica. Como se observa 
en la Figura 5.16 (a), la alimentación asimétrica mejora el comportamiento del sistema MZM linealizado ante HD3. Sin embargo, esta mejora en HD3 supone una disminución de $\mathrm{H}_{0}$, como indican los resultados de la Figura 5.16 (b). Este hecho indica que ante potencias bajas de la portadora modulada, no se consiguen transmisiones adecuadas, situándose incluso por debajo del nivel de ruido del sistema. Por tanto, es necesario llegar a situaciones de compromiso entre los valores de atenuación óptica y eléctrica para obtener suficiente señal de portadora $\mathrm{H}_{0}$ con valores HD3 aceptables.

Los resultados experimentales indican que el rango donde se mejora el HD3 en cerca de $20 \mathrm{~dB}$, es aquel donde el factor de atenuación RF se sitúa en un rango de 3.5 a $7 \mathrm{~dB}$, es decir, para valores de $\beta$ de 0.2 a 0.4 , y para un rango de atenuación óptica entre 18 y $25 \mathrm{~dB}$, o valores de $\alpha$ de 0.01 a 0.05 . Estos valores son comparables con los obtenidos analíticamente en la Figura 5.12.

Por otra parte, dependiendo de la aplicación final, es posible incrementar el margen dinámico de la arquitectura de linealización óptica DPMZM propuesta mediante la implementación de foto-detección balanceada, que se ha mostrado eficaz para reducir el ruido RIN del sistema [Jos05]. A su vez, las configuraciones de tipo push-pull en arquitecturas paralelas de linealización permiten mejorar el margen dinámico de todo el sistema [Gup07].

Finalmente, es necesario indicar que la técnica de linealización óptica DPMZM propuesta, se ha evaluado experimentalmente para un tono de $5 \mathrm{GHz}$, lo que se corresponde con las bandas de frecuencia de algunos de los servicios inalámbricos estudiados en esta Tesis Doctoral, como WiMAX Mobile.

\subsubsection{Derivas y efectos de segundo orden}

En secciones anteriores, se han descrito los efectos que las derivas en el punto de polarización producen en el comportamiento de los MZM. A su vez, también se ha destacado cómo el entorno de trabajo y la variación de las condiciones ambientales influyen en el comportamiento del MZM. Por estos motivos, en esta sección se presenta la evaluación de la arquitectura de linealización óptica DPMZM propuesta ante variaciones de la tensión de polarización, retardos temporales entre las diferentes ramas de alimentación asimétrica y ante variaciones de las condiciones ambientales de trabajo.

\subsubsection{Derivas en la alimentación asimétrica}

En la alimentación asimétrica, la señal moduladora RF recorre caminos físicos mediante cables o pistas, al igual que la potencia óptica. Sin embargo, estos caminos no suelen ser iguales, lo cual se traduce en retardos temporales y de fase en las señales, 
degradando el comportamiento del esquema de linealización. Estos retardos temporales y de fase se presentan en la ecuación (5.27).

$$
E_{\text {out }}(t)=E_{\text {in }}(t-\tau) e^{j \Delta \varphi}\left\{\begin{array}{c}
\Delta \varphi=-2 \pi f_{c} \tau \\
f_{c} \rightarrow \text { frecuencia_central }
\end{array}\right.
$$

Para evaluar la influencia de posibles derivas en la alimentación asimétrica, en primer lugar, se ha simulado el esquema de linealización óptica DPMZM propuesto con la herramienta de simulación óptica [VPI]; introduciendo retardos en las señales de RF que alimentan a cada MZM. La señal de RF utilizada está compuesta por dos tonos de $5 \mathrm{GHz}$ y $5.5 \mathrm{GHz}$ y por una amplitud de $1 \mathrm{Vpp}(0.5 \mathrm{~V})$. La alimentación asimétrica ideal se ha simulado mediante un valor de atenuación de RF de $8 \mathrm{~dB}$ y un valor de atenuación óptica de $6 \mathrm{~dB}$, lo cual supone unos $40 \mathrm{~dB}$ de rechazo de IMD3 en el esquema propuesto. La señal de RF tiene un periodo entre pulsos de 187.5 ps. Para simular el efecto del desfase eléctrico, se ha retardado la señal RF en una de las ramas de alimentación RF entre 0 y 187.5 ps.

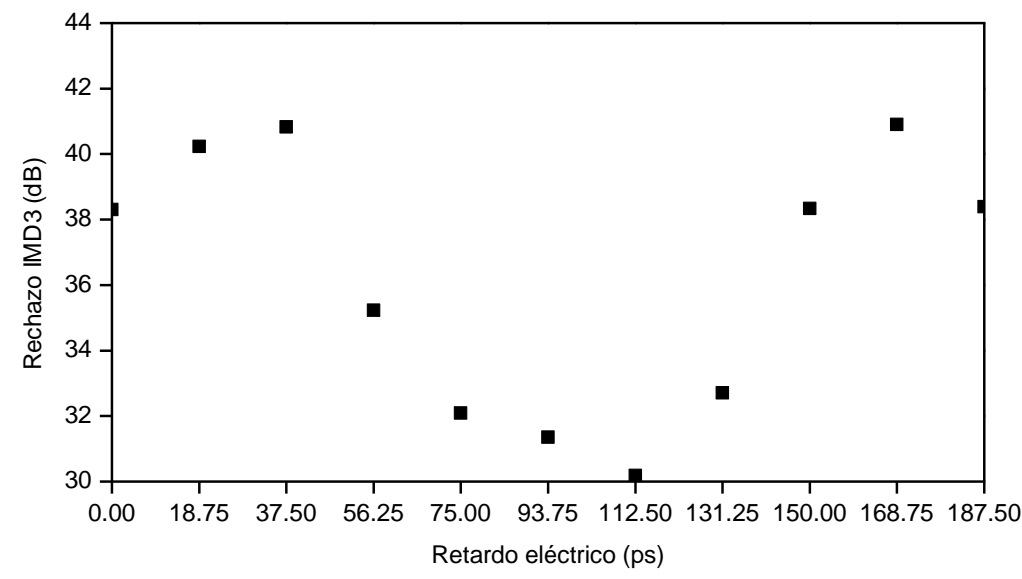

Figura 5.17. Resultados simulados de rechazo de IMD3 en la arquitectura de linealización óptica DPMZM propuesta, para retardos en una de las ramas de alimentación RF, con alimentación asimétrica basada en 6 dB de atenuación óptica y 8 dB de atenuación eléctrica.

En la Figura 5.17 se presentan los valores de IMD3 obtenidos en la simulación óptica. En este caso, se observa cómo el desfase eléctrico degrada la relación IMD3, alcanzando incluso valores inferiores a los de un único modulador MZM. 


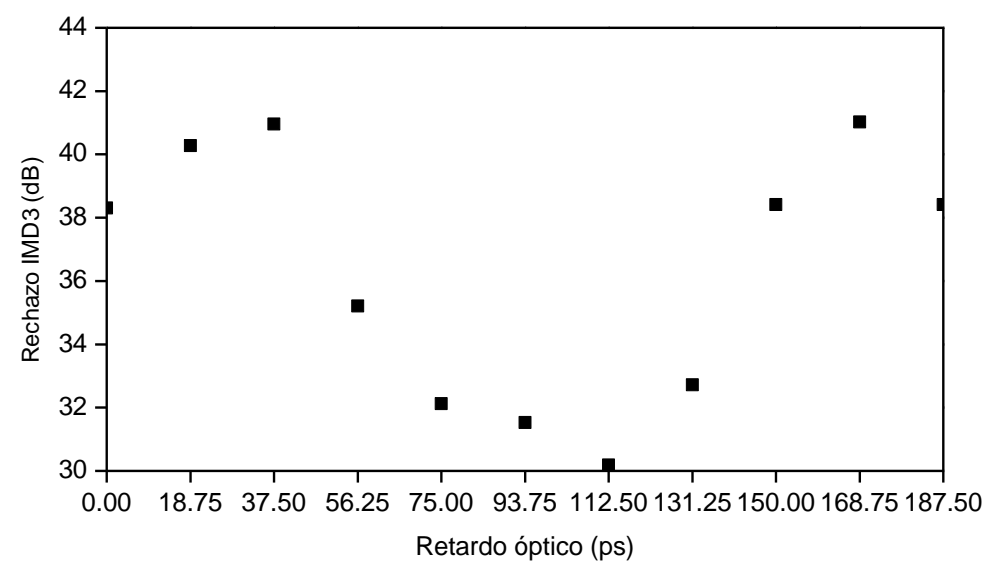

Figura 5.18. Resultados simulados de IMD3 en la arquitectura de linealización óptica DPMZM propuesta, para retardos en una de las ramas de alimentación óptica, con alimentación asimétrica basada en $6 \mathrm{~dB}$ de atenuación óptica y $8 \mathrm{~dB}$ de atenuación eléctrica.

En segundo lugar, se ha simulado el esquema de linealización óptica DPMZM propuesto introduciendo retardos en las señales ópticas que alimentan a cada MZM. En concreto, se han introducido retardos comprendidos entre 0 y $187.50 \mathrm{ps}$ en la rama óptica de salida del MZM\#2. Los parámetros de alimentación asimétrica se mantienen como en el estudio anterior, $6 \mathrm{~dB}$ de atenuación óptica y $8 \mathrm{~dB}$ de atenuación eléctrica. En la Figura 5.18 se presentan los valores de IMD3 obtenidos en la simulación óptica. Como se observa en la figura, el efecto del desfase óptico es muy similar al del desfase eléctrico presentado en la Figura 5.17.

Los resultados obtenidos en la simulación óptica, indican que los desfases en los caminos eléctricos y ópticos penalizan la técnica de linealización óptica DPMZM propuesta, con hasta $10 \mathrm{~dB}$ sobre los resultados IMD3 esperados.

Tras esta simulación, se lleva a cabo la evaluación experimental de la influencia de los retardos temporales entre las diferentes ramas de alimentación asimétrica. La evaluación experimental presenta ciertas dificultades, debido a que la longitud de los cables eléctricos, a la frecuencia de trabajo de $5 \mathrm{GHz}$, no introduce un desfase considerable en el montaje. Por ello, se ha optado por evaluar únicamente la influencia de los desfases de los caminos ópticos. En este caso, se ha introducido en el montaje experimental de la Figura 5.14 una línea de retardo óptico (ODL, Optical Delay Line) SANTEC-ODL-330, que introduce retardos en la fibra óptica de hasta 330 ps.

En el montaje experimental, ambos MZM se polarizan en QB y se genera una señal de RF con una frecuencia $5 \mathrm{GHz}$. Con la introducción del ODL, el armónico de segundo 
orden $\mathrm{H}_{2}$ aparece en las medidas realizadas con el RFSA, ya que su potencia se sitúa por encima del ruido del instrumento de medida. Los resultados experimentales han mostrado una degradación de $10 \mathrm{~dB}$ para la relación de rechazo de HD2 comparada con la evaluación experimental en condiciones óptimas. Sin embargo, los valores de rechazo de HD3 se mantienen con una degradación menor a 2 dB.

Estos resultados confirman la necesidad de diseñar correctamente los caminos eléctricos y ópticos en el sistema de linealización propuesto para minimizar el impacto del retardo eléctrico y óptico.

\subsubsection{Derivas en la tensión de polarización}

La deriva de la tensión de polarización o BIAS drift depende de las condiciones ambientales y parámetros de trabajo como son el tiempo de uso del modulador, la temperatura de trabajo y la longitud de onda de trabajo, como se explicó al principio de este capítulo. Los efectos principales de esta deriva son la variación del punto de polarización y del índice de modulación en el MZM.

En primer lugar, se ha evaluado mediante simulación óptica la deriva del BIAS para la técnica de linealización óptica DPMZM propuesta. En este caso, se ha configurado un rango de tensiones de polarización del 10\% sobre la tensión de polarización óptima. La alimentación asimétrica se ha simulado mediante un valor de atenuación de RF de $8 \mathrm{~dB}$ y un valor de atenuación óptica de $6 \mathrm{~dB}$. Se han generado dos señales RF de $5 \mathrm{GHz}$ y $5.5 \mathrm{GHz}$. El valor de QB en estos moduladores se sitúa en $V_{\pi}=3.5 \mathrm{~V}$, por lo tanto la tensión de polarización óptima es $1.75 \mathrm{~V}$. 

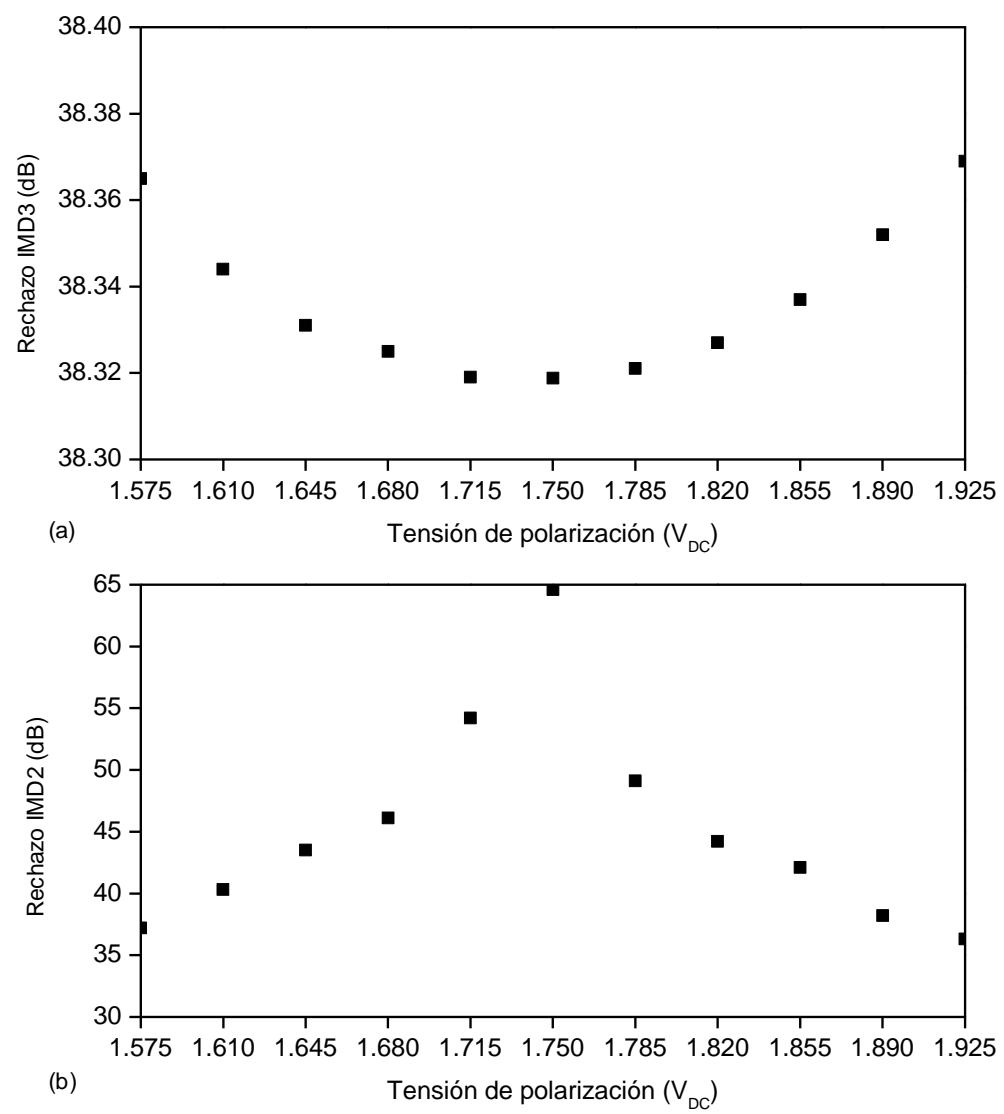

Figura 5.19. (a) Rechazo de IMD3 e (b) IMD2 en la arquitectura de linealización óptica DPMZM propuesta, para variaciones simuladas de la tensión de polarización.

En la Figura 5.19 se muestran los resultados IMD3 e IMD2 obtenidos en simulación óptica. La Figura 5.19 (b) muestra cómo aumenta el valor de rechazo IMD2 a medida que el valor de tensión de polarización se aleja del valor óptimo, con penalizaciones mayores a $20 \mathrm{~dB}$. Sin embargo, la Figura 5.19 (a) muestra una variación del rechazo IMD3 menor a $0.2 \mathrm{~dB}$ para el rango de tensiones de polarización simulado.

En un segundo paso, se ha evaluado mediante simulación óptica la influencia de una variación aleatoria de la tensión de polarización en cada MZM. Para ello se ha escogido una distribución Gaussiana con una deriva del 10\% sobre el valor óptimo de $1.75 \mathrm{~V}$, obteniendo los valores de tensión de polarización mostrados en la Tabla 5.4. 
TABLA 5.4. VALORES DE TENSIÓN DE POLARIZACIÓN PARA LA SIMULACIÓN DE LA DERIVA EN V $V_{\text {BIAS }}$

\begin{tabular}{l|c|c|c|c|c|c}
\hline \hline Medida número & $\mathbf{1}$ & $\mathbf{2}$ & $\mathbf{3}$ & $\mathbf{4}$ & $\mathbf{5}$ & $\mathbf{6}$ \\
\hline V $_{\text {BIAS }}$ MZM\#1 (V) & 2.0844 & 1.8246 & 1.8402 & 1.6039 & 1.6887 & 1.7677 \\
\hline V $_{\text {BIAS }}$ MZM\#2 (V) & 1.9667 & 1.7444 & 1.8630 & 1.6536 & 1.7861 & 1.5997 \\
\hline Medida número & & $\mathbf{7}$ & $\mathbf{8}$ & $\mathbf{9}$ & $\mathbf{1 0}$ & $\mathbf{1 1}$ \\
\hline$V_{\text {BIAS }}$ MZM\#1 (V) & 1.6001 & 1.5859 & 1.8177 & 1.8448 & 1.8351 \\
\hline$V_{\text {BIAS }}$ MZM\#2 (V) & 1.7263 & 1.7099 & 1.8295 & 1.9714 & 1.7941 \\
\hline \hline
\end{tabular}

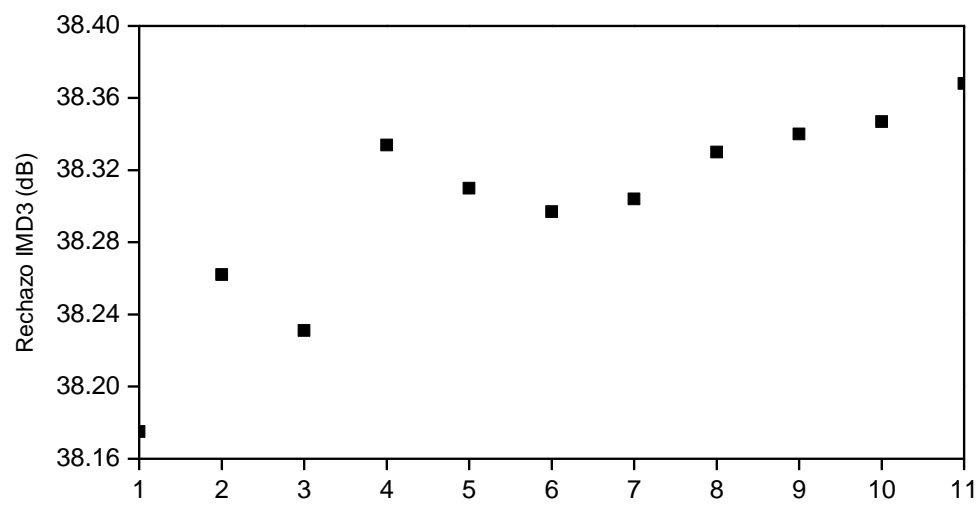

(a)

Medida de tensión de polarización

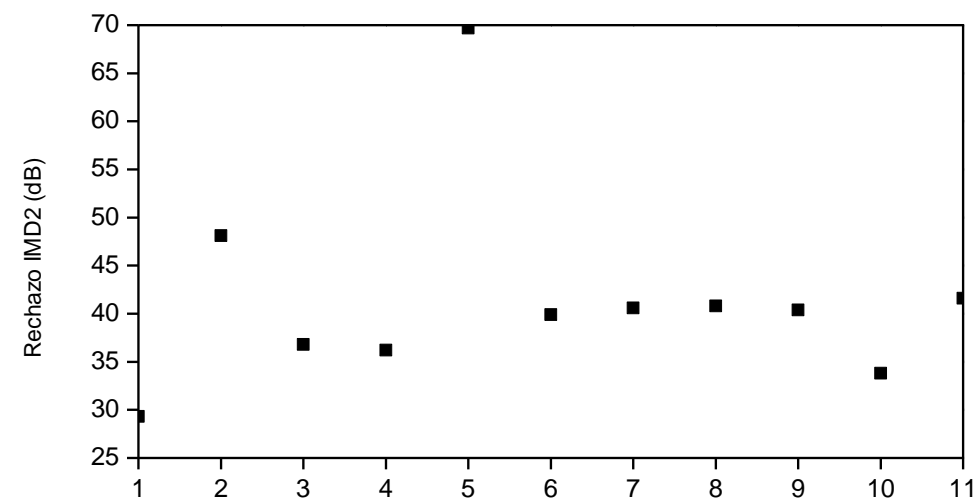

(b)

Medida de tensión de polarización

Figura 5.20. (a) Rechazo de IMD3 e (b) IMD2 en la arquitectura de linealización óptica DPMZM propuesta, para las variaciones simuladas de tensión de polarización mostradas en la Tabla 5.4.

En la Figura 5.20 se observan los resultados obtenidos en la simulación óptica de la arquitectura de linealización óptica DPMZM propuesta para los valores de tensión de polarización mostrados en la Tabla 5.4. La Figura 5.20 (a) muestra una variación del rechazo IMD3 menor a $0.2 \mathrm{~dB}$ para el rango de tensiones de polarización simulado. Sin 
embargo, los resultados de la Figura 5.20 (b) muestran cómo el rechazo IMD2 se convierte en una limitación del margen dinámico del sistema para ciertas combinaciones de valores de tensión de polarización.

Con respecto a la evaluación experimental de la influencia de la deriva de la tensión de polarización, es necesario comentar que la rápida variación de la tensión de polarización ha impedido analizar el efecto de estas derivas. Sin embargo, se ha comprobado que es necesario ajustar la tensión de polarización en ambos moduladores MZM al punto de trabajo QB tras tandas de medidas de duración menor a 30 minutos. Estos ajustes son de aproximadamente $\pm 0.05 \mathrm{~V}$ sobre el punto de trabajo QB inicial. De esta forma, se confirma la necesidad de disponer de mecanismos de control dinámico para el ajuste de la tensión de polarización, que permitan asegurar el correcto funcionamiento de los moduladores a lo largo de su vida útil.

\subsubsection{Deriva temporal}

A lo largo de la evaluación experimental de la técnica de linealización óptica DPMZM propuesta, se ha observado cómo la longitud de onda ha variado $0.001 \mathrm{~nm}$ tras una hora de trabajo. Esta desviación afecta al comportamiento de los moduladores MZM, puesto que parámetros como el punto de polarización o el índice de modulación dependen de la longitud de onda [Kel01].

La temperatura y el stress óptico no han sido condicionantes a lo largo de las evaluaciones experimentales realizadas. Los laboratorios en los cuales se han realizado las evaluaciones experimentales disponen de un control automático de la temperatura ambiente, lo que no ha permitido comprobar la dependencia del comportamiento de esta arquitectura con este parámetro. A su vez, la carga de trabajo sobre el esquema de linealización óptico DPMZM propuesto no ha sido tan intensa como para considerar el stress óptico como un condicionante en los resultados obtenidos. 
En este capítulo, se ha abordado el comportamiento no-lineal de los moduladores MZM y cómo afecta a la eficiencia de los sistemas RoF. Se ha definido este comportamiento no-lineal como una consecuencia de los materiales constructivos de los moduladores, del propio efecto de conversión electro-óptica, de la dependencia con la polarización, de las condiciones ambientales y de la dependencia con parámetros de trabajo que fluctúan con el tiempo, como la longitud de onda. Este comportamiento no-lineal se traduce en una limitación del margen dinámico de los MZM que degrada la eficiencia de modulación en aplicaciones analógicas donde el MZM es un elemento clave, como las redes de acceso integradas.

Se han revisado las técnicas que intentan mitigar este comportamiento, denominadas técnicas de linealización, que se clasifican en técnicas de linealización eléctricas y técnicas de linealización ópticas. Al mismo tiempo, es posible clasificar estas técnicas según sea el ancho de banda de trabajo, en técnicas de banda estrecha y técnicas de banda ancha.

En este capítulo, se propone y evalúa una técnica de linealización óptica de banda ancha, puesto que en esta Tesis Doctoral se ha evaluado la distribución y coexistencia de señales UWB y WiMAX, centradas en las bandas de frecuencias de $3.1 \mathrm{GHz}$ $-10.6 \mathrm{GHz}$ y $3.5 \mathrm{GHz}$, respectivamente. Por otro lado, este estudio se centra en una técnica de arquitectura paralelo porque teóricamente permite una mayor reducción de la distorsión IMD3, que es la que introduce una mayor distorsión de intermodulación en banda ancha.

El análisis de la técnica de linealización óptica DPMZM propuesta, ha mostrado mejoras de más de $20 \mathrm{~dB}$ de IMD3 sobre los resultados obtenidos con un solo modulador. La alimentación asimétrica utilizada en esta técnica conlleva limitaciones asociadas al ruido del sistema, puesto que en algunos casos la minimización de la distorsión de intermodulación del sistema requiere potencias ópticas muy bajas. Por ello, se plantea el uso de foto-detección diferencial para reducir el ruido RIN del sistema.

Por último, se ha evaluado la influencia de las derivas en tensión de polarización y en los retardos intrínsecos a la alimentación asimétrica del esquema. Los resultados obtenidos indican la necesidad de disponer de mecanismos de control dinámico de la tensión de polarización de los MZM y contemplar un diseño adecuado de los caminos eléctricos y ópticos en el sistema de linealización propuesto, para minimizar el impacto del retardo eléctrico y óptico.

Entre las aplicaciones de los MZM linealizados cabe destacar su aportación en el desarrollo de los convertidores analógico digitales mediante técnicas fotónicas [Llo7], donde su utilización permite el aumento del DR del sistema. Esto permite digitalizar 
señales de gran ancho de banda como WiMAX y UWB en tiempo real con márgenes dinámicos de hasta $50 \mathrm{~dB}$.

La técnica de linealización óptica DPMZM propuesta, se ha demostrado experimentalmente su validez para señales de banda ancha y en el rango de frecuencias utilizadas tanto por WiMAX como por UWB. 



\section{Capítulo 6. Conclusiones y líneas futuras}

\subsection{Conclusiones}

La actual demanda de servicios de elevado régimen binario en entornos residenciales y de negocios ha impulsado el desarrollo de nuevas tecnologías radio. A su vez, la necesidad por parte de los usuarios de disponer de un acceso móvil y ubicuo a los servicios requeridos, influye en la planificación y desarrollo de las redes de acceso. Por este motivo, la distribución integrada, tanto en la transmisión óptica como vía radiofrecuencia de señales radio en coexistencia, se considera como una solución adecuada para satisfacer la demanda requerida.

A lo largo de esta Tesis Doctoral se han propuesto nuevas técnicas para la coexistencia y distribución de señales inalámbricas en redes de acceso mediante sistemas RoF. Los sistemas de comunicación inalámbricos considerados son WiMAX y UWB. Estas dos tecnologías son altamente complementarias, pero sus canalizaciones en frecuencia dificultan su coexistencia espectral y su cobertura radio se solapa en escenarios de corta distancia. Por todos estos motivos, en esta Tesis Doctoral se ha estudiado y demostrado experimentalmente la convergencia inalámbrica de servicios basados en WiMAX y UWB sobre redes de acceso integradas.

En los capítulos 1 y 2 de esta Tesis Doctoral se ha revisado la arquitectura básica de las redes de acceso cableadas y vía radiofrecuencia. A su vez, se han introducido los aspectos más relevantes del uso de sistemas RoF en la implementación de redes de acceso integradas. Por último, se han descrito las tecnologías WiMAX y UWB, revisando a su vez las técnicas avanzadas de mitigación de la interferencia asociadas al despliegue de redes UWB. 


\subsubsection{Coexistencia radio de WiMAX y UWB}

En el capítulo 3 de esta Tesis Doctoral se ha evaluado experimentalmente la coexistencia inalámbrica de MB-OFDM UWB y WiMAX 802.16e en WPAN, en un escenario de tipo oficina. Las medidas experimentales realizadas han permitido obtener los valores de los márgenes de protección necesarios para asegurar la interoperabilidad entre WiMAX y UWB, de acuerdo con las técnicas de mitigación DAA.

En primer lugar, se ha evaluado el impacto de MB-OFDM UWB sobre un enlace de comunicaciones WiMAX 802.16e en el escenario propuesto. Los resultados obtenidos han mostrado que la robustez de una comunicación WiMAX 802.16e aumenta conforme aumenta su ancho de banda nominal. A su vez, se ha corroborado la mejora de la calidad del enlace WiMAX a medida que aumenta la distancia de separación entre el interferente MB-OFDM UWB y el enlace WiMAX 802.16e. Para el caso del menor ancho de banda nominal WiMAX 802.16e evaluado, se ha observado una penalización de $0.5 \mathrm{~dB}$ EVM en el enlace WiMAX por la presencia de un interferente MB-OFDM UWB, lo cual delimita un margen de seguridad de $2.5 \mathrm{~m}$ de separación entre ambos sistemas.

Posteriormente, se ha evaluado el impacto de WiMAX 802.16e sobre un enlace radio MB-OFDM UWB en el escenario propuesto. Los resultados obtenidos han demostrado que el enlace MB-OFDM UWB configurado con un TFC sin salto en frecuencia, presenta una degradación muy intensa debido a la presencia del interferente WiMAX, delimitando para este caso un margen de seguridad mayor de $10 \mathrm{~m}$. Sin embargo, la evaluación de un enlace MB-OFDM UWB configurado con un TFC con salto en frecuencia en presencia de un interferente WiMAX, delimita márgenes de seguridad de $5 \mathrm{~m}$ para garantizar la interoperabilidad entre ambos. A su vez, el aumento de ancho de banda nominal del interferente WiMAX 802.16e aumenta la degradación del enlace MB-OFDM UWB.

La evaluación de los resultados obtenidos de EVM UWB para cada subportadora del enlace MB-OFDM UWB ha mostrado que la degradación por la presencia del interferente WiMAX 802.16 siempre se localiza en un mismo rango de subportadoras. Esta información es de gran importancia para el desarrollo de técnicas avanzadas de mitigación de la interferencia, como DAA. Por ejemplo, se podría incorporar un filtro en tiempo real sobre este rango de subportadoras.

Finalmente, se ha evaluado el impacto de WiMAX 802.16e sobre el enlace MB-OFDM UWB cuando los valores de potencia transmitida superan el EIRP máximo UWB dado por la regulación existente. Los resultados obtenidos han mostrado una reducción de los márgenes de protección hasta distancias de $0.36 \mathrm{~m}$ para un 
EIRP UWB de $-35 \mathrm{dBm} / \mathrm{MHz}$. A su vez, se ha comprobado que para valores elevados de EIRP no se reducen los márgenes de protección, pero en cambio se produce un aumento de la distorsión del enlace debido a la saturación de los dispositivos MB-OFDM UWB del enlace.

En base a los resultados obtenidos en los distintos estudios experimentales, se puede concluir que la implementación de la coexistencia de UWB y WiMAX en escenarios WPAN necesita establecer los siguientes márgenes de protección:

- Enlace WiMAX 802.16e ante la presencia de un interferente MB-OFDM UWB: márgenes de protección superiores a $2.5 \mathrm{~m}$.

- Enlace MB-OFDM UWB ante la presencia de un interferente WiMAX 802.16e: márgenes de protección superiores a $5 \mathrm{~m}$ y a $10 \mathrm{~m}$, para los casos de TFC con salto en frecuencia y no salto en frecuencia, respectivamente.

- La implementación de técnicas de mitigación DAA para UWB en base a la información sobre el intervalo y número de subportadoras MB-OFDM UWB afectadas por el interferente WiMAX 802.16e, permitirá reducir los márgenes de protección.

- El aumento controlado de la potencia transmitida máxima EIRP de MB-OFDM UWB permitirá reducir los márgenes de protección, con el objetivo de asegurar la interoperabilidad en entornos WPAN menores a un metro.

\subsubsection{Distribución simultánea de WiMAX y UWB en sistemas RoF}

En el capítulo 4 de esta Tesis Doctoral se ha presentado una evaluación experimental de la distribución simultánea de UWB y WiMAX sobre sistemas RoF basados en fibra multimodo y monomodo.

En primer lugar, se ha evaluado experimentalmente la distribución simultánea de MB-OFDM UWB y WiMAX 802.16d sobre un sistema RoMMF. Los resultados obtenidos para el enlace MB-OFDM UWB han mostrado una degradación muy intensa debida tanto a la dispersión modal de la fibra MMF como a la presencia de WiMAX, limitando su alcance máximo radio. Sin embargo, los resultados obtenidos para el enlace WiMAX 802.16d han presentado una degradación menor y han mostrado la viabilidad de realizar la distribución conjunta de WiMAX 802.16d con MB-OFDM UWB sobre enlaces MMF de hasta $300 \mathrm{~m}$, con alcance máximo radio de $30 \mathrm{~m}$. Estos resultados han demostrado cómo la dispersión modal asociada a la fibra MMF es la principal limitación de los sistemas RoMMF cuando las señales UWB y WiMAX se distribuyen conjuntamente. A su vez, otra limitación importante en los 
sistemas RoMMF evaluados son las no-linealidades que presentan los dispositivos E/O, como los VCSEL o los foto-detectores PIN/TIA, ante la distribución de señales de frecuencias elevadas y grandes anchos de banda de trabajo como UWB y WiMAX.

En segundo lugar, se ha evaluado experimentalmente la distribución simultánea de MB-OFDM UWB y WiMAX 802.16e en sistemas RoSSMF. En un primer paso, se ha evaluado experimentalmente la distribución aislada de MB-OFDM UWB. Los resultados obtenidos han demostrado que la transmisión óptica sobre SSMF introduce una penalización en el alcance radio máximo de MB-OFDM UWB. A continuación, se ha evaluado la distribución simultánea de WiMAX y UWB en sistemas RoSSMF de tipo PON. En este caso, los resultados obtenidos muestran una penalización de $3 \mathrm{~dB}$ EVM para MB-OFDM UWB y de 2 dB EVM para WiMAX 802.16e para enlaces de hasta $25 \mathrm{~km}$ SSMF.

Posteriormente se han evaluado experimentalmente técnicas PDM en transmisión óptica para la distribución simultánea de WiMAX 802.16e y MB-OFDM UWB en sistemas RoSSMF PON. En concreto, se han propuesto dos estrategias PDM, una con interferencia cocanal en el espectro entre UWB y WiMAX y otra con interferencia de canal adyacente en el espectro entre ambos. Los resultados han demostrado que la estrategia PDM de canal adyacente presenta mejores prestaciones que la estrategia PDM cocanal en términos de EVM, ya que la interferencia residual de polarización entre UWB y WiMAX introduce una mayor distorsión en la estrategia PDM cocanal. Los resultados obtenidos han mostrado que para ambas estrategias es factible la distribución simultánea sobre enlaces de fibra SSMF de hasta $25 \mathrm{~km}$. A su vez, se ha comprobado cómo aumenta la degradación del enlace MB-OFDM UWB conforme aumenta el ancho de banda nominal WiMAX en ambas configuraciones.

Finalmente se ha demostrado cómo las técnicas PDM permiten aumentar la eficiencia espectral de los sistemas RoSSMF. Para ello se ha propuesto un esquema PDM en RoSSMF PON para la distribución dual de MB-OFDM UWB. Los resultados han mostrado la viabilidad de distribuir MB-OFDM UWB sobre enlaces de $25 \mathrm{~km}$ SSMF con un eficiencia espectral de $0.7576 \mathrm{bit} / \mathrm{s} / \mathrm{Hz}$ con tan solo una penalización de $2 \mathrm{~dB}$ respecto a una distribución MB-OFDM UWB en RoSSMF PON sin PDM.

En base a los resultados obtenidos en los distintos estudios experimentales, se puede concluir que para distribuir de forma simultánea UWB y WiMAX sobre sistemas RoF basados en fibra multimodo o monomodo, es necesario contemplar las siguientes limitaciones en el alcance y en sus prestaciones:

- La distribución simultánea de WiMAX 802.16d y MB-OFDM UWB en RoMMF es viable para señales WiMAX sobre enlaces de $300 \mathrm{~m}$ MMF con alcances radio de 
$30 \mathrm{~m}$. La dispersión modal introducida por la fibra MMF no permite la comunicación UWB.

- La distribución simultánea de WiMAX 802.16e y MB-OFDM UWB en RoSSMF: es viable para enlaces de $25 \mathrm{~km}$ SSMF PON.

- La utilización de técnicas PDM en transmisión óptica permite transmisiones de 25 km SSMF PON con una menor degradación de las comunicaciones, aumentando la eficiencia espectral del sistema.

- La utilización de técnicas PDM en transmisión óptica para la distribución dual de MB-OFDM UWB en RoSSMF duplica la eficiencia espectral hasta un valor de $0.7576 \mathrm{bit} / \mathrm{s} / \mathrm{Hz}$ sobre enlaces de $25 \mathrm{~km}$ SSMF PON.

\subsubsection{Linealización óptica de MZM para la distribución conjunta de WiMAX y UWB}

En el capítulo 5 de esta Tesis Doctoral se ha analizado la no-linealidad inherente a la función de transferencia del MZM, causante de la degradación del margen dinámico del sistema RoF ante señales RF con ancho de banda mayor a una octava, como son WiMAX y UWB.

En primer lugar, se han expuesto y revisado las principales técnicas de linealización de los moduladores MZM, en especial aquéllas denominadas técnicas de linealización óptica, puesto que no modifican la señal moduladora RF.

Posteriormente, se ha propuesto y demostrado experimentalmente una técnica de linealización óptica DPMZM de banda ancha. Esta técnica se basa en una arquitectura paralela con 2 MZM, incluyendo una alimentación de señal óptica y eléctrica asimétrica. La evaluación analítica de la arquitectura ha demostrado una mejora analítica del IMD3 de $30 \mathrm{~dB}$ sobre el obtenido en un MZM individual. La simulación óptica de la arquitectura ha mostrado una mejora de IMD3 de $25 \mathrm{~dB}$ para valores óptimos de alimentación eléctrica y óptica asimétrica. Finalmente, la evaluación experimental ha proporcionado resultados que muestran mejoras en torno a $20 \mathrm{~dB}$ para la IMD3. Por otra parte, los resultados experimentales han mostrado cómo la alimentación asimétrica propuesta en esta técnica conlleva limitaciones asociadas al ruido del sistema, puesto que en algunos casos la minimización de la distorsión de intermodulación requiere potencias ópticas muy bajas, lo cual aumenta el ruido en la señal óptica modulada. En este caso, se ha propuesto el uso de foto-detección diferencial para reducir el ruido RIN del sistema. 
Finalmente, se ha demostrado experimentalmente la influencia de las derivas en tensión de polarización y en los retardos intrínsecos a la alimentación asimétrica de la técnica de linealización óptica DPMZM de banda ancha propuesta. Los resultados obtenidos han demostrado la necesidad de disponer de mecanismos de control dinámico de la tensión de polarización de los MZM y de realizar un diseño adecuado de los caminos eléctricos y ópticos para minimizar el impacto del retardo eléctrico y óptico.

Estos resultados concluyen que la técnica de linealización óptica DPMZM de banda ancha propuesta, permite aumentar el margen dinámico del sistema RoF para la coexistencia e integración de comunicaciones inalámbricas UWB y WiMAX. 


\subsection{Líneas futuras}

A continuación se indican las líneas futuras que se podrían desarrollar a partir de los resultados obtenidos en esta Tesis Doctoral:

- La implementación práctica de técnicas DAA mediante filtrado en tiempo real de las subportadoras MB-OFDM UWB afectadas. Esta implementación permitiría comprobar la efectividad de DAA y mostrar su viabilidad como una solución para el adecuado despliegue de MB-OFDM UWB en entornos WPAN. Paralelamente, conllevaría la implementación de algoritmos que reorganicen la información de las subportadoras filtradas en el resto de subportadoras de la señal MB-OFDM UWB.

- El estudio de soluciones de bajo coste para la convergencia inalámbrica de servicios basados en UWB y WiMAX en redes basadas en sistemas RoF. Este estudio se podría dividir en dos etapas. En primer lugar, la evaluación de láseres VCSEL en $1550 \mathrm{~nm}$ para la distribución simultánea de UWB y WiMAX en redes de acceso basadas en sistemas RoSSMF. En segundo lugar, el estudio de la utilización de fibra multimodo de plástico POF.

- La evaluación de la extensión de la convergencia inalámbrica en sistemas RoF a través de una implementación real para la distribución de múltiples servicios como WLAN, UMTS, WiMAX, Bluetooth, UWB, Zigbee, y LTE. Este estudio permitiría cuantificar la complejidad que supone la gestión y distribución simultánea de estas señales RF.

- El análisis de la extensión de la convergencia inalámbrica y la distribución simultánea de UWB y WiMAX en redes de acceso basadas en sistemas RoF a otros servicios basados en OFDM, como el futuro estándar LTE. Este estudio permitiría evaluar el impacto del despliegue de servicios quíntuple-play mediante redes basadas en sistemas RoF con fibra MMF, SSMF o de tipo POF.

- La evaluación de la fabricación de un dispositivo MZM compacto en base a la técnica de linealización propuesta en esta Tesis Doctoral. Esta comprobación experimental permitiría analizar el impacto del uso de MZM linealizados en un sistema RoF real con distribución simultánea de UWB y WiMAX. 



\section{Bibliografía}

[3GPP] 3rd Generation Partnership Project (3GPP), 3GPP standards release 7 "Technical Specifications and Technical Reports for a UTRANbased 3GPP system," http://www.3gpp. org/article/release-7.

[Ack99] E.I. Ackerman, "Broad-band linearization of a Mach-Zehnder electro optic modulator" IEEE Transactions on Microwave Theory and Techniques, vol. 46 no. 12, pp. 2271-2279, Diciembre 1999.

[Agr02] G.P. Agrawal, Fiber-Optic Communication Systems, 3rd Edition, Wiley-Interscience; 2002.

[Alv09a] T. Alves, A. Cartaxo, "Performance Degradation Due to OFDM-UW B Radio Signal Transmission Along Dispersive Single-Mode Fiber," IEEE Photonics Technology Letters, vol. 21, no. 3, pp. 158-160, Febrero 2009.

[ALV09b] Alvarion BreezeMAX Si CPE (Self-installable, indoor subscriber unit), http://www.alvarion.com/solutions/privatenetwork/products/breezemax/.

[ALV09c] Alvarion Ltd., Mobile WiMAX Solutions, 4MotionTM Open WiMAX Solution, http://www.alvarion.com/solutions/mobile/.

[Are06] C. Arellano, C. Bock, J. Prat, and K. Langer, "RSOA-based optical network units for WDM-PON," in Proc. Optical Fiber Communication Conference OFC06, paper OTuC1, 2006.

[ARIB] Association of Radio Industries and Businesses of Japan, http://www .arib.or.jp 
[Bac03] L. Bach, W. Kaiser, J.P. Reithmaier, A. Forchel, T.W. Berg, B. Tromborg, "Enhanced direct-modulated bandwidth of $37 \mathrm{GHz}$ by a multi-section laser with a coupled-cavity-injection-grating design," IEE Electronics Letters, vol. 39, no. 22, pp. 1592-1593, Octubre 2003.

[Bat03] A. Batra et al., "Multi-band OFDM physical layer proposal for IEEE 802.15 task group 3a,” IEEE P802.15-03/268r3, Julio 2003.

[Bel08] M. Beltrán, M. Morant, J. Pérez, R. Llorente, J. Martí, "Photonic generation and frequency up-conversion of impulse-radio UWB signals," in Proc. 21 st Annual Meeting of the IEEE Lasers and Electro-Optics Society LEOS2008, pp. 498-499, 2008.

[Bel09] Marta Beltran, Roberto Llorente, Rakesh Sambaraju, Javier Marti, "60 GHz UWB-over-fiber system for in-flight communications," in Proc. IEEE MTT-S International Microwave Symposium Digest MTT09, pp. 5-8, Junio 2009.

[Ben04] M.G. di Benedetto, G. Giancola, Understanding ultra-wideband radio fundamentals, Prentice Hall PTR, 2004.

[Ben08] Y. Ben Ezra, B.I. Lembrikov, M. Ran, A. Leibovich, E. Borohovich, "Experimental and theoretical investigation of the multiband OFDM ultra wideband radio over multimode fiber transmission," in Proc. 2008 IEEE International Conference on Ultra-Wideband ICUWB2008, vol. 3, pp. 55-58, 2008.

[Ben78] C. L. Bennett, G. F. Ross, "Time-domain electromagnetics and its applications," Proceedings of IEEE, vol. 66, no. 3, pp. 299-318, 1978.

[Ber95] H.L. Bertoni, L. Carin, L.B. Felsen, Ultra-Wideband, Short-Pulse Electromagnetics, Kluwer Academic/ Plenum Press, 1995.

[Bet02] G. Betts, "LiNbO3 external modulators and their use in high performance analog links," RF Photonic Technology in Optical Fiber Links, ch 4, W. Chang, Cambridge University Press, 2002.

[Bet94] G.E.Betts, "Linearized modulator for suboctave-bandpass optical analog links," IEEE Transactions on Microwave Theory and Techniques, vol. 42, pp. 2642-2649, Diciembre 1994.

[BLU09] WiMedia Alliance Transfers Specification, http://www.bluetooth. com/Bluetooth/Technology/Technology_Transfer/.

[Boh06] C. Bohm, "Video QoS in triple-play networks," Lightwave Online Magazine, Febrero 2006.

[BONE] Building the Future Optical Network in Europe: The e-Photon/ONe Network (BONE), funded under Seventh Framework Programme through FP7-ICT-FP7-216863. 
[Bri92] G. J. McBrien, J. D. Farina, Cascaded optic modulator arrangement, US patent 5168534, Diciembre 1992.

[Bur95] W.K. Burns, "Linearized Optical modulator with fifth order correction," IEEE Journal of Lightwave Technology, vol. 13, no. 8, pp. 1724-1727, Agosto 1995.

[Bur99] W.K. Burns, M.M. Howerton, R.P. Moeller, R. Krahenbuhl, R.W. McElhanon,A.S. Greenblatt, "Low Drive Voltage, Broad-Band LiNbO3 Modulators with and without Etched Ridges," IEEE Journal of Lightwave Technology, vol. 17, no. 12, pp. 2551-2555, Diciembre 1999.

[Cap07] J. Capmany, D. Novak, "Microwave photonics combines two worlds" Nature Photonics, vol. 1, no. 6, pp. 319-330, Junio 2007.

[Car02] C. Carlsson, H. Martinsson, R. Schatz, J. Halonen, A. Larsson, "Analog modulation properties of Oxide Confined VCSELS at microwave frequencies," IEEE Journal of Lightwave Technology, vol. 20, no. 9, pp. 1740-1749, Septiembre 2002.

[Cas03] A. Casini, P. Faccin, "Wavelength Division. Multiplication Technologies for UMTS Radio. Coverage extension by using the radio over fibre technique," in Proc. IEEE International Topical Meeting on Microwave Photonics MWP03, pp. 123-128, 2003.

[Cas05] D. Cassioli, S. Persia, V. Bernasconi, A. Valent, "Measurements of the performance degradation of UMTS receivers due to UWB emissions," IEEE Communications Letters, vol. 9, no. 5, pp.441-443, Mayo 2005.

[Cha06] P. Chanclou, S. Gosselin, J.F. Palacios, V.L. Alvarez, E. Zouganeli, "Overview of the optical broadband access evolution: a joint article by operators in the IST network of excellence e-Photon/One," IEEE Communications Magazine, vol. 44, no. 8, pp. 29 - 35, Agosto 2006.

[Cha08a] Q. Chang, J. Gao, Q. Li, Y. Su, "Simultaneous Transmission of Point -to-Point Data and Selective Delivery of Video Services in a WDMPON Using ASK/SCM Modulation Format," in Proc. Optical Fiber Communication Conference OFC08, paper OWH2, Febrero 2008.

[Cha08b] Q. Chang, Y. Tian, T. Ye, J. Gao, Y. Su, “A 24-GHz ultra-wideband over fiber system using photonic generation and frequency up-conversion," IEEE Photonics Technology Letters, vol. 20, no. 19, pp. 1651-1653, Octubre 2008.

[Chi03] M. Chia, B. Luo, M.L. Yee, E.J.Z. Hao, "Radio over multimode fibre transmission for wireless LAN using VCSELs," IEE Electronics Letters, vol. 39, no. 15, pp. 1143-1144, 2003. 
[Chi99] Y. Chiu, B. Jalali, "Broad-band electronic linearizer for externally modulated analog fiber-optic links," IEEE Photonics Technology Letters, vol. 11, no. 1, pp. 48-50, Enero 1999.

[CMT09] Comisión del Mercado de las Telecomunicaciones, "Nota mensual Febrero 2009," España, 2009.

[Con99] J.T. Conroy, J.L. Lo Cicero, D.R. Ucci, "Communication techniques using monopulse waveforms," in Proc. IEEE Military Communications Conference MILCOM99, vol. 2, pp. 1181-1185, Noviembre 1999.

[Cor01] J. L. Corral, J. Martí, J. M. Fuster, "General expressions for IM/DD dispersive analog optical links with external modulation or optical upconversion in a Mach-Zehnder electrooptical modulator," IEEE Transactions on Microwave Theory and Techniques, vol. 49, no. 10, Part 2, pp. 1968-1976, Octubre 2001.

[Cor09] J.L. Corral, R. Llorente, V. Polo, B. Vidal, J. Martí, J. Porcar, D. Zorrila, A.J. Ramírez, "Application of Radio-Over-Fiber in WiMAX: Results and Prospects," WiMAX Evolution, pp 385-400, John Wiley \& Sons Ltd., 2009.

[Cot06] M. Cotton, et al., "Interference Potential of Ultrawideband Signals Part 3: Measurement of UWB Interference to C Band Satellite Digital Television Receivers," NTIA Report TR 06 437, Febrero 2006.

[Cox04] Charles H. Cox III, Analog Optical Links: Theory and Practice, Cambridge University Press, 2004.

[Cri09] M.J. Crisp, S. Sabesan, R.V. Penty, "Radio over Fiber Distributed Antenna Networks," IEEE LEOS Newsletter, vol. 23, no. 1, pp. 16-20, Febrero 2009.

[Cum98] U. V. Cummings, W. B. Bridges, "Bandwidth of Linearized Electrooptic Modulators," IEEE Journal of Lightwave Technology, vol. 16 no. 8, pp. 1482-1490, Agosto 1998.

[Dag99] N. Dagli, "Wide-bandwidth lasers and modulators for RF photonics," IEEE Transactions on Microwave Theory and Techniques, vol. 47, no. 7, pp. 1151-1171, 1999.

[Dar08] T. Darcie, J. Zhang, "High performance microwave-photonic links, ” in Proc in Proc. IEEE Radio and Wireless Symposium RWS, 2008, pp. 125-128, 2008.

[Das06] A. Das, A. Nkansah, N.J. Gomes, I.J. Garcia, J.C. Batchelor, D. Wake, "Design of low-cost multi-mode fiber-fed indoor wireless networks," IEEE Transactions on Microwave Theory and Techniques, vol. 54, no. 8, pp. 3426-3432, Agosto 2006. 
[DEC09] (2009/343/CE) DECISIÓN DE LA COMISIÓN de 21 de abril de 2009 que modifica la Decisión 2007/131/CE por la que se autoriza la utilización armonizada del espectro radioeléctrico para los equipos que utilizan tecnología de banda ultraancha en la Comunidad.

[Des87] E. Desurvire, J. Simpson, P. C. Becker, "High-gain erbium-doped traveling-wave fiber amplifier," Optics Letters, vol. 12, pp. 888-890, 1987.

[Des94] E. Desurvire, Erbium Doped Fibre Amplifiers, John Wiley \& sons Ltd., 1994.

[Djo07] B. Djordjevic, "PMD compensation in fibre-optic communication systems with direct detection using LDPC-coded. OFDM," Optics Express, vol. 15, no. 7, pp. 3692-3701, 2007.

[ECC06.04] ECC/DEC/(06)04: On the harmonised conditions for devices using ultra-wideband (UWB) technology in bands below $10.6 \mathrm{GHz}$, European Commission, Marzo 2006.

[ECC07.02] ECC/DEC/(07)02: ECC Decision of 30 March 2007 on availability of frequency bands between $34003800 \mathrm{MHz}$ for the harmonized implementation of Broadband Wireless Access systems (BWA), 2007.

[ECC08.10] ECC Decision of 1 December 2006 amended Cordoba, 31 October 2008 on supplementary regulatory provisions to Decision $\mathrm{ECC} / \mathrm{DEC} /(06) 04$ for UWB devices using mitigation techniques (ECC/DEC/(06)12), amended 31 Octubre 2008.

[ECC120] ECC Report 120 on Technical requirements for UWB DAA (DetectAnd-Avoid) devices to ensure the protection of Radiolocation in the bands $3.1-3.4 \mathrm{GHz}$ and $8.5-9 \mathrm{GHz}$ and BWA terminals in the band 3.4 - $4.2 \mathrm{GHz}$, Junio 2008.

[ECMA05] ECMA International, presentation on "High Rate - Ultra Wide Band (UWB) Background, ” 2005, http://www.ecma-international.org/activ ities/Communications/tg20_UWB_Background.pdf.

[ECMA368] ECMA 368 International Standard, High rate ultra wideband PHY and MAC Standard, 3rd Edition, Diciembre 2008.

[ECMA369] ECMA-369 International Standard, MAC-PHY Interface for ECMA-368, 3rd Edition Diciembre 2008.

[EFM]

IEEE 802.3 Ethernet Working Group. IEEE P802.3ah Ethernet in the First Mile, http://www.ieee802.oeg/3/ah/index.html.

[E-photon/one] Optical Networks: Towards Bandwidth Manageability and Cost Efficiency (e-Photon/ONe+), funded under Sixth Framework Programme through FP6-IST-027497. 
[Ess09] R.-J. Essiambre, G. Foschini, P. Winzer, G. Kramer, "Capacity limits of fiber-optic communicaction systems," in Proc. Optical Fiber Communication Conference OFC09,paper OThL1, 2009.

[ETSI] European Telecommunications Standard Institute, http://www.etsi.org

[ETSI08] ETSI EN 302065 V1.1.1 (2008-02): Electromagnetic compatibility and Radio spectrum Matters (ERM); Ultra WideBand (UWB) technologies for communication purposes; Harmonized EN covering the essential requirements of article 3.2 of the R\&TTE Directive, Febrero 2008.

[Fac09] F. Facchini, R. Giuliano, F. Mazzenga, "Ultra-wideband detect and avoid procedure for WiMAX victims," IET Communications, vol. 3, no. 2, pp. 268-278, Febrero 2009.

[Far02] J.O. Farmer, "Delivering video, voice and data to consumers via al all-fiber network," IEEE Transactions on Consumer Electronics, vol. 48, pp. 548-555, 2002.

[Far08] J.O. Farmer, K. Bourg, "Practical Deployment of Passive Optical Networks," IEEE Communications Magazine, pp. 136-145, Junio 2008.

[FCC] Federal Communications Commission, http://www.fcc.gov

[FCC02.48] FCC 02-48, Revision of Part 15 of the Commission's Rules Regarding Ultra-Wideband Transmission Systems, First Report and Order, ET Docket 98-153, Abril 2002.

[Fla07] A. Flatman, "In-premises optical fiber installed base analysis to 2010, Presentation to the IEEE 802.310 Higher Speed Study Group, Ottawa (Canada), Abril, 2007.

[Foe03] J. R. Foerster et al., "Channel modeling sub-committee report final," IEEE 802.15-02/490, Noviembre 2003.

[FRA09] Franklin Wireless Inc. WiMAX and 3G USB Modem CMU-300 / EVDO Rev. A + WiMAX, http://www.franklinwireless.com

[FREDIT] Desarrollo de un demostrador tecnológico de procesador fotónico para receptores electrónicos digitales (FREDIT), proyecto financiado por el gobierno de España a través de FIT-330100-2006-95.

[Fri98] N.J. Frigo, P.P. Iannone, K.C. Reichmann, "Spectral slicing in WDM passive optical networks for local access," in Proc. 24th European Conference on Optical Communication ECOC98, vol. 1, pp.119-120, Septiembre 1998.

[FSAN] The Full Service Access Network (FSAN) Group. http://www.fsanweb.org. 
[Fu08]

[Gen07]

[Geo04]

[Giu05]

[Giu08]

[Gle84]

[Gna07]

[Gon09]

[Gro08]

[GSA09]

[Gu06]
S. Fu, W. Zhong, Y. J. Wen, P. Shum, "Photonic monocycle pulse frequency up-conversion for ultrawideband-over-fiber applications," IEEE Photonics Technology Letters, vol. 20, no. 12, pp. 1006-1008, Junio 2008.

N. Genay, P. Chanclou, F. Saliou, Q. Liu, T. Soret, L. Guillo, "Solutions for Budget Increase for the Next Generation Optical Access Network," in Proc. International Conference on Transparent Optical Networks ICTONO7, vol. 1, pp. 317-320, Julio 2007.

A. Georgiadis, "Gain, phase imbalance, and phase noise effects on error vector magnitude," IEEE Transactions on Vehicular Technology, vol. 53, no. 2, pp. 443-449, Marzo 2004.

R.Giuliano, F. Mazzenga, "On the coexistence of power controlled ultrawide band systems with UMTS, GPS, DCS1800, and fixed wireless systems," IEEE Transactions on Vehicular Technology, vol. 54, no. 1, pp. 62-81, Enero 2005.

R. Giuliano, F. Mazzenga, "Impact of detect and avoid in UWB regulation process," in Proc. IEEE International Conference on Ultra-Wideband ICUWB2008, vol. 3, pp. 67-70, Septiembre 2008.

G.V. Glebovich, A.V. Andriyanov, V. Vvedenskij, I.P. Kovalev, V.V. Krylov, A. Ryabinin, "Study of Objects Using Picosecond Pulses," Radio i Svyaz', 1984.

A.H. Gnauck, G. Charlet, P. Tran, P. Winzer, C. Doerr, J. Centanni, E. Burrows, T. Kawanishi, T. Sakamoto, K. Higuma, “ 25.6 TB/s $\mathrm{C}+\mathrm{L}-$ band transmission of polarization multiplexed RZDQPSK signals," in Proc. Optical Fiber Communication Conference OFC07, paper PDP19, 2007.

Ignacio González Insua, Dirk Plettemeier, Christian G. Schäffer, "Broadband radio-over-fiber-based wireless access with $10 \mathrm{Gbits} / \mathrm{s}$ data rates," Journal of Optical Networking, vol. 8, no. 1, pp. 77-83, Enero 2009.

K. Grobe, J.-P. Elbers, "PON in adolescence: from TDMA to WDMPON," IEEE Communications Magazine, vol. 46, no. 1, pp. 26-34, Enero 2008.

Global mobile Suppliers Association, GSA REPORT TO 3GPP PCG \#22, 2009.

X. Gu, W. Mohammed, P. Smith, "Demonstration of all-fiber WDM for multimode fiber local area networks," IEEE Photonics Technology Letters, vol. 18, no. 1, pp. 244-246, Enero 2006. 
[Guo08] Y.X. Guo; V.H. Pham; M.L Yee; L.C. Ong; B. Luo, "Performance evaluation for WiMedia Ultra-wideband simultaneous transmission with wireless LAN over cable and fiber," in Proc. IEEE Radio and Wireless Symposium RWS08, pp. 259-262, Enero 2008.

[Gup07] S. Gupta, B. Jalali, "2nd Order Distortion Cancellation in Photonic Time Stretch Analog-to-Digital Converter," in Proc. IEEE MTT-S International Microwave Symposium Digest MTT07, pp. 229-232, Junio 2007.

[Ham03] M. Hamalainen, J. Saloranta, J.-P. Makela, I. Oppermann, T. Patana, "Ultra wideband signal impact on IEEE802.11b and Bluetooth performances," in IEEE Proceedings on Personal, Indoor and Mobile Radio Communications PIMRC03, vol. 3, pp. 2943-2952, Septiembre 2003.

[Har03] P. Hartmann, M. Webster, A. Wonfor, J.D. Ingham, R.V. Penty, I.H. White, D Wake, A. J. Seeds, "Low-cost multimode fibre-based wireless LAN distribution system using uncooled directly modulated DFB laser diodes," in Proc. European Conference on Optical Communication ECOC03, vol. 3, pp. 804-805, 2003.

[Har81] H. F. Harmuth, Nonsinusoidal Waves for Radar and Radio Communication, Academic Press, New York, NY, USA, 1981.

[Has97] R. Hassun, M. Flaherty, R. Matreci, M. Taylor, "Effective evaluation of link quality using error vector magnitude techniques," in Proc. Wireless Communications Conference 1997, pp. 89-94, Agosto 1997.

[Hel00] J.C: Twichell, R.J. Helkey, "Linearized optical sampler" US Patent 6028424, 22 Febrero 2000

[HIT09] HITACHI WOO LCD TV Series, http://www.hitachi.com

[Hu07] S. Hu, G. Wu, Y.L. Guan, C.L. Law, Y. Yan, S. Li, "Development and performance evaluation of mobile WiMAX testbed," in Proc. IEEE Mobile WiMAX Symposium 2007, pp. 104-107, Marzo 2007.

[IEEE80211] The Working Group for WLAN Standards. IEEE 802.11TM Wireless Local Area Networks, http://www.ieee802.org/11/.

[IEEE80211g] IEEE 802.11g-2003: Further Higher Data Rate Extension in the 2.4 $\mathrm{GHz}$ Band, http://standards.ieee.org/getieee802/download/802.11g-2 003.pdf.

[IEEE80215.1] IEEE 802.15 WPAN Task Group 1 (TG1) IEEE 802.15.1 Standard for Wireless Personal Area Networks, http://www.ieee802.org/15/pu b/TG1.html

[IEEE80215.3] IEEE 802.15 WPAN Task Group 3 (TG3) IEEE 802.15.3 Standard for High Rate Wireless Personal Area Networks, http.//www.ieee802 .org/15/pub/TG3.html 
[IEEE80215.4] IEEE 802.15 WPAN Task Group 4 (TG4) IEEE 802.15.4 Standard for Low Rate Wireless Personal Area Networks, http://www.ieee802. org/15/pub/TG4.html

[IEEE80216a] IEEE 802.16 Working Group on Broadband Wireless Access Standards. IEEE 802.16 WirelessMAN Standard for Wireless Metropolitan Area Networks, http://www.ieee802.org/16/

[IEEE80216b] IEEE Standard Conformance to "IEEE Std 802.16 Part 3: Radio Conformance Tests (RCT) for 10-66 GHz Wireless MAN-SC Air Interface," 2004.

[IEEE80216d] IEEE Std 802.16TM-2004, "802.16 ${ }^{T M}$ IEEE standard for local and metropolitan area networks Part 16: Air interface for fixed broadband wireless access systems," Octubre 2004.

[IEEE80216e] IEEE Std 802.16e-2005; IEEE Standard for Local and metropolitan area networks; Part 16: Air Interface for Fixed Broadband Wireless Access Systems, Amendment 2: Physical and Medium Access Control Layers for Combined Fixed and Mobile Operation in Licensed Bands and Corrig. 1, Febrero 2006.

[INT09] Intel® Centrino $® 2$, www.Intel.com/es/centrino2

[ISIS] Infrastructures for broadband access in wireless/photonics and integration of strengths in Europe (ISIS) funded under Sixth Framework Programme through EC CONTRACT N 26592.

[ISO11801] ISO/IEC 11801 Information technology - Generic cabling for customer premise Standard, 1995.

[ISO26907] ISO/IEC 26907:2007 - Information technology-Telecommunications and information exchange between systems - High Rate Ultra Wideband PHY and MAC Standard, 2007.

[ISO26908] ISO/IEC 26908:2007 - Information technology - MAC-PHY Interface for ISO/IEC 26907, 2007.

[ITUG694.1] ITU-T recommendation G.694.1, Spectral grids for WDM applicattions: DWDM frequency grid, 2002.

[ITUG959.1] ITU-T Recommendation G.959.1, Optical transport networks physical layer interfaces, 2003.

[ITUG983] ITU-T G.983 series: Broadband optical access systems based on Passive Optical Networks (PON), 2001, http://www.itu.int/rec/T-RE C-G/en

[ITUG984] ITU-T G.984 series: Gigabit-capable Passive Optical Netwroks (GPON), 2003, http://www.itu.int/rec/T-REC-G/en 
[ITUM1457] ITU, Recommendation ITU-R M.1457. Detailed Specifications of the Radio Interfaces of International Mobile Telecommunications-2000 (IMT-2000), 2007.

[Jan07a] S.L. Jansen, I. Morita, N. Takeda, H. Tanaka, "20-GB/s OFDM transmission over 4,160 km SSMF enabled by RF-pilot tone phase noise compensation," in Proc. Optical Fiber Communication Conference OFC07, PDP15, 2007.

[Jan07b] K. A. Janjua, S. A. Khan, "A Comparative Economic Analysis of different FTTH Architectures," in Proc. International Conference on Wireless Communications, Networking and Mobile Computing WiCom07, pp. 4979 4982, 2007.

[Jaz08] M. Jazayerifar, B. Cabon, J.A. Salehi, "Transmission of Multi-Band OFDM and Impulse Radio Ultra-Wideband Signals Over Single Mode Fiber," IEEE Journal of Lightwave Technology, vol. 26, no.15, pp. 2594-2603, Agosto 2008.

[Jen08] J.B. Jensen, X. Yu, I.T. Monroy, C. Peucheret, P. Jeppesen, "Combined Transmission of Baseband NRZ-DQPSK and Phase Modulated Radio-over-Fibre," in Proc. European Conference on Optical Communication ECOC2008, Septiembre 2008.

[Joh01] L.A. Johansson, A.J. seeds, "36-GHz 140-Mb/s radio-over-fiber transmission using an optical injection phase-lock loop source," IEEE Photonics Technology Letters, vol. 13., no. 8, pp-893-895, 2001.

[Jos05] A. Joshi, X. Wang, D. Mohr, D. Becker, C. Wree, "Balanced photoreceivers for analog and digital fiber optic communications," in Proceedings of SPIE 5814, pp. 39-50, 2005.

[Kah06] J. Kahn, "Modulation and Detection Techniques for Optical Communication Systems," in Proc. Optical Amplifiers and Their Applications/Coherent Optical Technologies and Applications, Technical Digest OAA/COTA06, paper CThC1, 2006.

[Kao66] C. Kao, G. Hockman, "Dielectric-fiber dielectric fiber waveguides for optical frequencies," in Proceedings of IEEE, vol. 133, no. 3, pp. 1155-1158, 1966.

[Kas08] A. Kaszubowska-Anandarajah, P. Perry, L.P. Barry, "Hybrid radio over fiber system for generation and distribution of UWB signals," in Proc. International Conference on Transparent Optical Networks ICTON08, vol. 4, pp. 82-85, Junio 2008.

[Kat09] M. Katz, F. Fitzek, WiMAX Evolution: Emerging Technologies and Applications, John Wiley and Sons Ltd., 2009 
[Kaw01] H. Kawanishi, Y. Yamauchi, N. Mineo, Y. Shibuya, H. Mural, K. Yamada, H. Wada, "EAM-integrated DFB laser modules with more than $40 \mathrm{GHz}$ bandwidth," IEEE Photonic Technology Letters, vol. 13, no. 9, pp. 954-956., Septiembre 2001.

[Kaw02] Ivan P. Kaminow, Tingye Li, Optical Fiber Telecommunications IVA, Academic Press, 2002.

[Kaw03] T. Kawanishi, M. Izutsu, "Suppression of optical harmonics in wavelength conversion using optical single-sideband modulator," in Proc. Optical Fiber Communication Conference OFC03, vol. 2, pp. 771- 772, Marzo 2003.

[Kaz07] L.G. Kazovsky, W.-T. Shaw, D. Gutierrez, N. Cheng, S.-W. Wong, "Next-Generation Optical Access Networks," IEEE Journal of Lightwave Technology, vol. 25, no. 11, pp. 3428-3442, Noviembre 2007.

[Kel01] P.V. Kelkar, A.S. Bhushan, A. Krishnaswami, S. Yegnanarayanan, S.A. Pappert, B. Jalali “Effect of Mach-Zehnder modulator's spectral response on the time-stretch analog-to-digital converter," in Proc. Conference on Lasers and Electro-Optics CLEO01, paper CMN3, Mayo 2001.

[Kim09] G. Kim, “Paradigm shift in access markets?,” Febrero 2009, http://w ww.ipbusinessmag.com/departments.php?department_id=7\&article_ $\mathrm{id}=483$.

[Kob08] A. Kobyakov, D. Thelen, A. Chamarti, M. Sauer, J. Winter, "MIMO radio signals over fiber in picocells for increased WLAN coverage," in Proc. Optical Fiber Communication Conference OFC08, paper JWA113, 2008.

[Koh04] R. Kohno, "State of Arts in Ultra Wideband (UWB) Wireless Technology and Global Harmonization," in Proc. European Microwave Conference euMC04, vol. 2, pp. 1093 1099, Octubre 2004.

[Koo02] A. Koonen, H. van den Boom, F. Willems, J. Bergmans, G. Khoe, "Broadband multiservice in-house networks using mode group diversity multiplexing," in Proc. International Conference on Plastic Optical Fibers POF02, pp. 87-90, 2002.

[Koo03] A. Koonen, A. N'gOma, H. van den Boom, I. Tafur Monroy, G. Khoe,'New tecniques for extending the capabilities of multimode fibre networks," in Proc. European Conference on Networks \& Optical Communications NOC03, pp. 204-211, 2003. 
[Koo07] T. Koonen, M. García Larrodé, P. Urban, H. Waardt, C. Tsekrekos, J.Yang, H.Yang, H. Boom, "Fiber-based Versatile Broadband Access and In-Building Networks," IET Workshop: From Access to Metro@Broadband Europe 2007, Diciembre 2007.

[Koo08] A.M.J. Koonen,M. Garcia Larrode, "Radio-over-MMF techniques Part II: Microwave to millimeter-wave systems," invited paper in IEEE Journal of Lightwave Technology, Vol.26, No.15, pp. 2396-2408, Agosto 2008.

[Kor90] Steven K. Korotky, René M. de Ridder, "Dual Parallel Modulation Schemes for Low-Distortion Analog Optical Transmission," IEEE Journal on Selected Areas in Communications, vol. 8, no. 7, pp. 1377-1381, 1990.

[Kur06] T. Kuri, Y. Omiya, T. Kawanishi, S. Hara, K. Kitayama, “Optical transmitter and receiver of 24-GHz ultra-wideband signal by direct photonic conversion techniques," in Proc. IEEE International Topical Meeting on Microwave Photonics MWP06, pp. 1-4, 2006.

[Lar06]

M. Larrode, A. Koonen, J. Olmos, A. Ng'Oma, "Bidirectional radioover-fiber link employing optical frequency multiplication," IEEE Photonics Technology Letters, vol. 18, no. 1, pp. 241-243, 2006.

[Lee05] S.-M. Lee, K.-M. Choi, S.-G. Mun, J.-H. Moon, C.-H. Lee, "Dense WDM-PON based in wavelength-locked fabry perot laser diodes," IEEE Photonic Technology Letters, vol. 17, no. 7, pp. 1579-1581, Julio 2005.

[Lee06] C.-H. Lee, W.V. Sorin, B.Y. Kim, "Fiber to the Home Using a PON Infrastructure," IEEE Journal of Lightwave Technology, vol. 24, no. 12, pp. 4568-4583, Diciembre 2006.

[Leg07] Y. Le Guennec, R.Gary, "Optical frequency conversion for millimeter-wave ultra-wideband-over-fiber systems," IEEE Photonics Technology Letters, vol. 19, no. 13, pp. 996-998, Julio 2007.

[LEN09] Lenovo ThinkPad W Series, http://www.lenovo.com

[Lin86] L. Linnell, “A Wideband Local Access System Using Emerging -Technology Components," IEEE Journal on Selected Areas in Communications, vol. 4, no. 4, pp. 612-18, Julio 1986.

[Llo07] R. Llorente, J. Pérez, M. Beltrán, J. Martí, “Convertidores analógico digitales fotónicos: Tecnología y aplicaciones," XVII Jornadas TELECOM I+D 2007,2007. 
[Llo08a] R. Llorente, J. Perez, M. Beltran, M. Morant, J. Martí, "UWB picocell clusters: real time interference monitoring," in Proc. ICT Mobile and Wireless Communications Summit ICT-Mobile Summit08, June 2008.

[Llo08b] R. Llorente, T. Alves, M. Morant, M. Beltran, J. Perez, A. Cartaxo, J. Marti, "Ultra-Wideband Radio Signals Distribution in FTTH Networks," IEEE Photonics Technology Letters, vol. 20, no. 11, pp. 945-947, Junio 2008.

[Llo09] ICT-UCELLS FP7-2007-ICT-1-216785, R. Llorente, J. Perez, M. Beltrán, M. Morant, J. Duplicy, D. Meshulam, Public Deliverable D7.2 - "First report on the UWB technology evolution," Enero 2009.

[Low05] A.J. Lowery, J. Armstrong, "10 Gbit/s multimode fibre link using power efficient orthogonal frequency division multiplexing," Optics Express, vol. 13, pp. 10003-10009, 2005.

[Lun06] T. Lunttila et al., "Advanced coding schemes for a multiband OFDM ultrawideband system towards 1 Gbps," in Proc. IEEE Consumer Communications and Networking Conference CCNCO6, pp. 553 557, January 2006.

[Luo05] B. Luo, L.C. Ong, M.L. Yee, Y.X. Guo, M.Y.W. Chia, "Centralized UWB/WLAN distribution network using low cost radio over multimode fiber technology," in Proc. IEEE Vehicular Technology Conference VTC05-Fall, vol. 2, pp. 799-801, Septiembre 2005

[Mar02] T. Marozsak, E. Udvary, "Vertical cavity surface emitting lasers in radio over fiber applications," in Proc. International Conference on Microwaves, Radar and Wireless Communications MIKONO2, vol. 1, pp. 41-44, 2002.

[Mar07] F. Martínez, J. Campos, A. Ramírez, V. Polo, A. Martínez, D. Zorrilla, J. Martí, “Transmission of IEEE802.16d WiMAX signals over radio-over-fibre IMDD links," in Proc. International Conference on Wired/Wireless Internet Communications WWIC07, May 2007.

[Mar99] J. Marti, F. Ramos, V.Polo, J.M.Fuster, J.L.Corral, "Millimetre-wave signal generation and harmonic upconversion through PM-IM convesion in chirped fiber gratings," in Proc. IEEE International Topical Meeting on Microwave Photonics MWP99, pp. 181-184, 1999.

[Mat97] Y. Matsui, H. Murai, S. Arahira, S. Kutsuzawa, Y. Ogawa, "30-GHz bandwidth $1.55 \mu \mathrm{m}$ strained-compensated INGAALAS-INGAASP MQW laser," IEEE Photonics Technology Letters, vol. 9, no. 1, pp. 25-27, Enero 1997. 
[MBC05] Modulator Bias Controllers for LN MZM, MBC-LN, Photline Tech., http://www.photline.fr/MBC.pdf

[Mce94] T.E: McEwan, Ultra-wideband radar motion sensor, US Patent 5,361,070, 1 Noviembre 1994.

[Mea87] R. J. Mears, L. Reekie, I. M. Jauncey, D. N. Payne, "Low-noise Erbium doped fibre amplifier at $1.54 \mathrm{pm}$, , IEE Electronics Letters, vol. 23, pp. 126-1028, 1987.

[Mei05] H. H. Meinel, J. Wenger, H. Henftling, G: Rollmann H. Dominik, "Automotive radar: From long range collision warning to short range urben amployment," in Proc. International Joint Conference of the MINT 6th Millimeter-Wave International Symposium (and the $7^{\text {th }}$ Topical Symposium on Millimeter Wave MINT-MISO5 TSMMW05, pp. 244-247, 2005.

[Mis07a] S.M. Mishra, R.W. Brodersen, S.T. Brink, R. Mahadevappa, "Detect and avoid: an ultra-wideband/WiMAX coexistence mechanism [Topics in Radio Communications]," IEEE Communications Magazine, vol. 45, no. 6, pp. 68-75, Junio 2007.

[Mis07b] S.M. Mishra, R.W. Brodersen, "Cognitive Technology for improving Ultra-Wideband (UWB) Coexistence," in

Proc. IEEE International Topical Meeting on Microwave Photonics MWP07, pp. 253-258, Septiembre 2007.

[Mit95] O. Mitomi, K. Noguchi, H. Miyazawa, "Design of ultra-broad-band LiNbO3 optical modulator with ridge structure," IEEE Transactions on Microwave Theory and Techniques, vol. 43 no. 9, pp. 2203-2207, Septiembre 1995.

[Mit99] J. Mitola III, G.Q. Maguire Jr., "Cognitive radio: making software radios more personal," IEEE Personal Communications, vol. 6, no.4, pp. 13-18, Agosto 1999.

[Mon08] L.-A. de Montmorillon, G. Kuyt, P. Nouchi, A. Bertaina, "Latest advances in optical fibers," Comptes Rendus Physique, vol. 9, no. 9-10, pp. 1045-1054, 2008.

[MOT09] Motorola's wi4 WiMAX USBw 100, http://www.motorola.com/ wimax

[Nag01] Y. Nagasawa et alt., "High performance dispersion compensating fiber module," Fujikura Technical Review, no. 20, pp. 1-7, 2001

[Nak08] M. Nakazawa, "Challenges to FDM-QAM coherent tranmssion with ultrahigh spectral effiency," in Proc. European Conference on Optical Communication ECOCO8, paper Th3.E.4., 2008. 
[Nav02] M.D. Nava, C. Del-Toso, "A short overview of the VDSL system requirements," IEEE Communications Magazine, vol. 40, no. 12, pp. 82-90, Diciembre 2002.

[Naz93] M. Nazarathy, J. Berger, J. Ley, M. Levi, and Y. Kagan, "Progress in externally modulated AM CATV transmission systems," IEEE Journal of Lightwave Technology, vol. 11, pp. 82-105, Enero 1993.

[Naz94] M. Nazarathy, Y. Kagan, Y. Simler, Cascaded optical modulation system with high linearity, US Patent no. 5278923, 11 Enero 1994.

[Nik08] H. Nikookar, R. Prasad, Introduction to Ultra Wideband for Wireless Communications, 1st Edition, Springer Publishing Company, Inc., 2008.

[Nua07] L. Nuaymi, WiMAX: Technology for Broadband Wireless Access, John Wiley \& sons, 2007.

[Oga92] H. Ogawa, D. Polifko, S. Samba, "Millimter.wave fiber optic system s for personal radio communication," IEEE Transactions on Microwave Theory and Techniques, vol. 40, no. 12, pp. 2285-2292, Diciembre 1992.

[OLI09] Wireless USB Adapter Set OLIDATA, http://www.olidata.com/wusb

[Ols98] N.A. Olsson, "Lightwave Systems with optical amplifiers," IEEE Journal of Lightwave Technology, vol. 7, pp. 1071-1082, 1989.

[Ong06] L.C. Ong, M.L. Yee, B. Luo, "Transmission of ultra wideband signal through radio-over-fiber systems," in Proc. Annual Meeting of the IEEE Lasers and Electro-Optics Society LEOSO6, pp. 522-523, Octubre 2006.

[Pai07] A. Paier et al., "First results from car-to-car and car-to-infrastructure radio channel measurements at $5.2 \mathrm{GHz}$," in IEEE Proceedings on Personal, Indoor and Mobile Radio Communications PIMRC07, pp. 1-5, 2007.

[Par04] S.-J. Park, C.-H. Lee, K.-T. Jeong, H.-J. Park, J.-G. Ahn, K.H. Song, "Fiber-to-the-Home Services Based on Wavelength-DivisionMultiplexing Passive Optical Network," IEEE Journal of Lightwave Technology, vol. 22, no. 11, pp. 2582-2591, Noviembre 2004.

[Pep05] P. Pepeljugoski, "Next generation high-speed multimode fiber links and their specifications," in Proc. Optical Fiber Communication Conference OFC05, vol. 3, pp. 149-174, 2005.

[Per06] K.-A Persson, C. Carlsson, A. Alping, A. Haglund, J.S. Gustavsson, P. Modh, A. Larsson, "WCDMA radio-over-fibre transmission experiment using singlemode VCSEL and multimode fibre," IEE Electronics Letters, vol. 42, no. 6, pp. 372-374, Marzo 2006. 
[Pol04] V. Polo, G. Gómez, A. Martínez, J. Herrera, J. Martí, "Photonicmicrowave harmonic mixers based on electroabsorption modulator," Microwave and Optical Technology Letters, vol. 41, n. 5, pp. 361-364, Junio 2004.

[Pol99a] V. Polo, J. Martí, F. Ramos, D. Moodie, "Mitigation of chromatic dispersion effects employing electroabsorption modulator-based transmitters," IEEE Photonics Technology Letters, vol. 11, no. 7, pp. 883-885, Julio 1999.

[Pol99b] V. Polo, F. Ramos, J. Marti, J.M. Fuster, "Demonstration of dispersion-tolerant $34 \mathrm{Mbit} / \mathrm{s}$ data transmission in electro-optically upconverted $28 \mathrm{GHz}$ LMDS fiber-optic link," in Proc. IEEE MTT-S International Microwave Symposium Digest MTT99, vol. 3, pp. 1205-1208, 1999.

[Por03] D. Porcino, "Coexistence of UWB technology with FWA services," in Proc. European Microwave Conference euMC03, vol. 2, pp. 857-860, Octubre 2003.

[Pra05a] J.Prat, C. Arellano, V. Polo and C. Bock, "Optical network unit based on bidirectional reflective semiconductor optical amplifier for fiber-to-the-home networks," IEEE Photonics Technology Letters, vol. 17, no. 1, pp. 250-252, Enero 2005.

[Pra05b] J. Prat, "High-Density Passive Fiber-to-the-Home Networks," in Proc. International Conference on Transparent Optical Networks ICTON05, vol. 2, pp. 33-37, 2005.

[Pra06] J. Prado et al., "The MBOA-WiMedia specification for ultra wideband distributed networks," IEEE Communications Magazine, vol. 44, no. 6, pp. 128 - 134, Junio 2006.

[Pra96] R. Prasad, S. Hara, “An overview of multi-carrier CDMA," in Proc. IEEE International Symposium on Spread Spectrum Techniques and Applications ISSSTA96, vol. 1, pp. 107-114, Septiembre 1996.

[Rab02] P. Rabiei, W.H. Steier, Z. Cheng, L.R. Dalton, "Integrated WDM polymer modulator," in Proc. Optical Fiber Communication Conference OFC02, pp. 31-33, Marzo 2002.

[Rad97] L. Raddatz, I. White, D. Cunningham, M. Nowell, M. Tan, S. Wang, "Fiber-optic m-ary modulation scheme using multiple light sources," in Proc. Optical Fiber Communication Conference OFC97, pp. 198-199, Febrero 1997.

[RAE] Real Academia Española de la Lengua, Diccionario Panhispánico de dudas - Primera edición (octubre 2005), "Acepción de Punto," http:// buscon.rae.es/dpdI/SrvltConsulta?lema=punto 
[Rah07]

[Rah08]

[Ram98]

[Ran07]

[Rer07]

[Roe08]

[Ros73]

[Sal87a]

[Sal87b]

[San08]

[Sau06]
A. Rahim, S. Zeisberg, A. Finger, "Coexistence Study between UWB and WiMAX at $3.5 \mathrm{GHz}$ Band," in Proc. IEEE International Conference on Ultra-Wideband ICUWB07, pp. 915 920, Septiembre 2007.

A. Rahim Biswas, F. Berens, S. Zeisberg, A. Finger, "Determination of time domain mitigation parameters for coexistence of WiMedia and WiMax systems," in Proc. IEEE International Conference on Ultra-Wideband ICUWB08, vol. 3, pp. 59-62, Septiembre 2008.

R. Ramaswami, K.N. Sivarajan, Optical Networks: A practical Perspective. The Morgan Kaufmann Series in Networking, Morgan Kaufmann Publishers, Inc. San Francisco, 1998.

Moshe Ran, Yossef Ben Ezra, Motti Haridim, Boris. I. Lembrikov, "Ultra Wideband Radio over Optical Fiber," in Proc. Wireless World Research Forum 2007 WWRF07, White Paper presentation WG5, pp. 1-40, 2007.

L. Rerko, Triple-Play Service Deployment: A Comprehensive Guide to Test,Measurement, and Service Assurance, JDS Uniphase Corporation, 2007.

G. Roelkens, D. Vermeulen, D. Van Thourhout, R. Baets, S. Brision, P. Lyan, P. Gautier, J.-M. Fedeli, "High efficiency diffractive grating couplers for interfacing a single mode optical fiber with a nanophotonic silicon-on-insulator waveguide circuit," Applied Physics Letters, vol. 92,no. 13, pp. 131101-131103, Marzo 2008.

G. Ross, Fundamental patent on UWB communications U.S. Patent No.3.728.632, Abril 1973.

A. M. Saleh, R. A. Valenzuela, "A statistical model for indoor multipath propagation," IEEE Journal on Selected Areas in Communications, vol. 5, no. 2, pp. 128-137, 1987.

A. Saleh, A. Rustako, R. Roman. "Distributed antennas for indoor radio communications," IEEE Transactions on Communications, vol. 35, no. 12, pp. 1245-1251, Diciembre 1987.

A. Sano, E. Yamada, H. Masuda, E. Yamazaki, T. Kobayashi, E. Yoshida, Y. Miyamoto, S. Matsuoka, R. Kudo, K. Ishihara, Y. Takatori, M. Mizoguchi, K. Okada, K. Hagimoto, H. Yamazaki, S. Kamei, H. Ishii, “13.4-Tb/s (134 × 111-Gb/s/ch) no-guard-interval coherent OFDM transmission over 3,600 km of SMF with 19-ps average PMD," in Proc. European Conference on Optical Communication ECOCO8, pp. 1-2, Septiembre 2008.

M. Sauer, Communication Systems for the Mobile Information Society, John Wiley \& sons Ltd., Septiembre 2006. 
[Sau07] M. Sauer, A. Kobyakov, J. George, "Radio Over Fiber for Picocellular Network Architectures," IEEE Journal of Ligthwave Technology, vol. 25, no. 11, pp. 3301-3320, Noviembre 2007.

[Sch08] K. Schuh, B. Franz, B. Junginger, P. Klose, E. Lach, "Serial 107 Gbit/s (2x53.5 Gbit/s NRZ-VSB-Polmux) transmission over $15 \mathrm{~km}$ SSMF with electronic dispersion compensation," in Proc. European Conference on Optical Communication ECOC08, September 2008.

[Sch95] H. Schmuck, "Comparison of optically millimeter-wave system concepts with regard to chromatic dispersion," IEE Electronics Letters, vol. 31, no. 21, pp. 1848-1849, 1995.

[See06] A.J. Seeds, "Microwave Photonics," IEEE Journal of Lightwave Technology, vol. 24, no. 12, pp. 4628-4641, 2006.

[Sha05] A. Shah, R. Hsu, A. Tarighat, A. Sayed, B. Jalali, "Coherent optical MIMO (COMIMO)," IEEE Journal of Lightwave Technology, vol. 23, no. 8, pp. 2410-2419, 2005.

[Sha06] R.A: Shafik, S. Rahman, I. AHM Razibul, “On the Extended Relationships Among EVM, BER and SNR as Performance Metrics” in Proc. International Conference on Electrical and Computer Engineering ICECE06, pp. 408-411, Diciembre 2006.

[Shi00] S. Shimotsu, S. Oikawa, T. Saicou, N. Mitsugi, K. Kubodera, T. Kawanishi, M. Izutsu, "LiNbO3 optical single-sideband modulator," in Proc. Optical Fiber Communication Conference OFC02, vol. 4, pp. 214-217, 2000.

[Shi01] S.Shimotsu, S. Oikawa, T. Saicou, N. Mitsugi, K. Kubodera, T. Kawanishi, M. Izutsu, "Single side-band modulation performance of a LiNbO3 integrated modulator consisting of four-phase modulator waveguides," IEEE Photonics Technology Letters, vol.13, no. 4, pp. 364-366, Abril 2001.

[Shu08] P.W. Shumate, "Fiber-to-the-Home: 1977-2007," IEEE Journal of Lightwave Technology, vol. 26, no. 9, pp. 1093-1103, Mayo 2008.

[Sim05] C.K. Sim, M.L. Yee, B. Luo, C.L. Ong, M.Y.W. Chia, "Performance evaluation for wireless LAN, Ethernet and UWB coexistence on hybrid radio-over-fiber picocells," in Proc. Optical Fiber Communication Conference OFC0, vol. 3, Marzo 2005.

[Ske91] H. Skeie, R. V. Johnson, "Linearization of electro-optic modulators by a cascade coupling of phase modulating electrodes," in Proceedings of SPIE 1583, pp. 156-164, 1991.

[Smi98] G. H. Smith, D. Novak, C. Lim, “A millimetre-wave full-duplex WDM-SCM fiber radio access network," in Proc. Optical Fiber Communication Conference OFC98, paper TuC5, 1998. 
[Sno07]

[Ste07]

[Ste87]

[Sti97]

[Sto99]

[Str05]

[Stu00]

[Tan05]

[TOPRATE] Terabit/s optical transmission systems based on ultra-high channel bitrate (TOPRATE), funded under Fifth Framework Programme through IST-2000-28657.

[Tsu01] T. Tsuritani, I. Morita, N. Edagawa, "Ultra long-haul transmission with multi-terabit capacity," in Proc. Annual Meeting of the IEEE Lasers and Electro-Optics Society LEOSO1., vol. 1, pp. 151-152, 2001.

[Tyl02] E. Tyler, M. Webster, R. Penty, I. White, S. Yu, J. Rorison, "Subcarrier modulated transmission of $2.5 \mathrm{~Gb} / \mathrm{s}$ over $300 \mathrm{~m}$ of 62.5 um-core diameter multimode fiber," IEEE Photonics Technology Letters, vol. 14, no. 12, pp. 1743-1745, 2002. 
[UCELLS] Ultra-wideband real-time interference monitoring and cellular management strategies (UCELLS), funded under Seventh Framework Programme through FP7-2007-IST-1-216785.

[Ulm07] J. Ulm, B. Weeks, "Next play evolution: Beyond triple play \& quad play," in Proc. IEEE International Symposium on Consumer Electronics ISCE2007, pp. 1-6, 2007.

[UROOF] Photonic components for ultra wideband radio over optical fiber (UROOF), funded under Sixth Framework Programme through FP6-2005-IST-5-033615.

[USB09] USB-IF - WiMedia Alliance Technology Transfer, http://www.usb.or g/press/WiMedia_Tech_Transfer/

[VPI] VPItransmissionMaker ${ }^{\mathrm{TM}}$ Optical Systems; Virtual Photonics, Inc.

[Wag89] S. S. Wagner, H. Kobrinski, "WDM Applications in Broadband Telecommunication Networks," IEEE Communications Magazine, vol. 27, no. 3, pp. 22-30, Marzo 1989.

[Wak02a] D. Wake, "Trends and prospects for radio over fibre picocells," in Proc. IEEE International Topical Meeting on Microwave Photonics MWP02, pp. 21-24, Noviembre 2002.

[Wak02b] D. Wake, S. Dupont, J. Vilcot, A. Seeds, “32-QAM radio transmission over multimode fibre beyond the fibre bandwidth in Proc. IEEE International Topical Meeting on Microwave Photonics MWP01, vol. Supplement, p.4, Enero 2002.

[Wan92] Y. Wang-Boulic, "A linearized optical modulator for reducing thirdorder intermodulation distortion," IEEE Journal of Lightwave Technology, vol. 10, no. 8, pp. 1066-1070, Agosto 1992.

[Way99] W.I. Way, Broadband hybrid Fiber/Coax access system technology, Academic Press, 1999.

[Wib05] A. Wiberg, P. Perez-Millan, M.V. Andres, P.A. Andrekson, P.O. Hedekvist, "Fiber-optic 40-GHz mm-wave link with $2.5-\mathrm{Gb} / \mathrm{s}$ data transmission," IEEE Photonics Technology Letters, vol. 17, no. 9, pp. 1938-1940, Septiembre 2005.

[Wil97] G. C. Wilson, "Optimized predistortion of overmodulated Mach-Zehnder modulators with multicarrier input," IEEE Photonics Technology Letters, vol. 9, pp. 1535-1537, Noviembre 1997.

[Wim] WiMAX Forum, http://www.wimaxforum.org

[Wim08] WiMAX Forum, WiMAX Technology Forecast, 2008, http://www.wi maxforum.org/technology/downloads/wimax_forum_wimax_forecas ts_6_1_08.pdf. 
[Win05] P.J. Winzer, G. Raybon, M. Duelk, “107-Gb/s optical ETDM transmitter for 100G ethernet transport," in Proc. European Conference on Optical Communication ECOC05, paper Th4.1.1., 2005.

[Xie03] X. Xie, J. Khurgin, J. Kang, F.-S. Chow, "Linearized Mach-Zehnder intensity modulator," IEEE Photonics Technology Letters, vol. 15, no. 4, pp. 531-533, Abril 2003.

[Yan04] C. Yang, S: Lee, J. Wu, "Optical-isolator-based modules for monitoring DWDM tunable lasers," Journal of Optical Networking, vol. 3, no. 6, pp. 452-463, Junio 2004.

[Yee07] M.L. Yee, Y.X. Guo, V.H. Pham, L.C. Ong, "WiMedia Ultra-Wide Band Transmission in Radio over Fiber using Multimode Fiber," in Proc. Annual Meeting of the IEEE Lasers and Electro-Optics Society LEOS07, pp. 335-336, Octubre 2007.

[Yu06] J. Yu, Z. S. Jia, L. Xu, L. Chen, T. Wang, G. K. Chang, “DWDM optical millimeter-wave generation for radio-over-fiber using an optical phase modulator and an optical interleaver," IEEE Photonics Technology Letters, vol. 18, no. 13, pp. 1418-1420, Julio 2006.

[Yue04] R. Yuen, X. Fernando, S. Krishnan, "Radio over multimode fiber for wireless access," in Proc. Canadian Conference on Electrical and Computer Engineering, vol. 3, pp. 1715-1718, 2004. 